\title{
CIVILIAN APPLICATIONS OF LASER FUSION
}

J. Maniscalco, J. Blink, R. Buntzen, J. Hovingh,

W. Meier, M. Monsler, and P. Walker

November 17, 1977

\section{MASTER}

Work performed under the auspices of the U.S. Department of Energy by the UCLLLL under contract number W-7405-ENG-48.

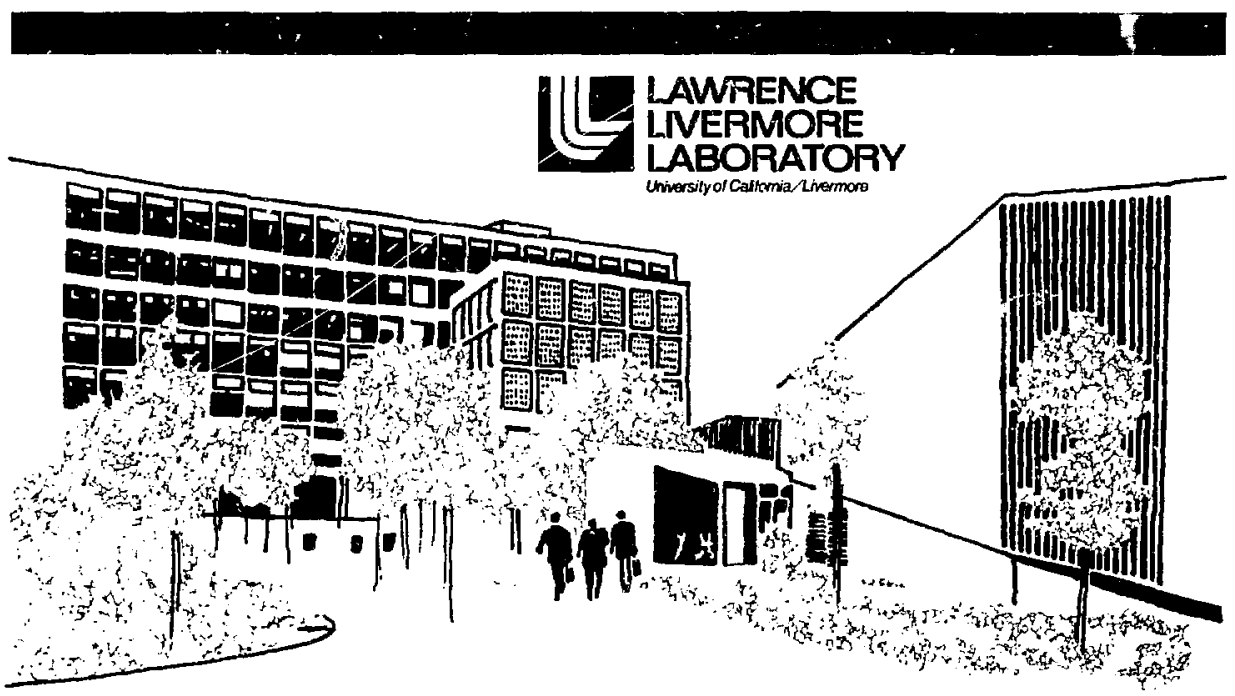




\section{近 \\ LAWRENCE LJVERMORE LABORATORY}

University of Cahtomia Livermore,California 94550

\section{MASTER}

UCRL-52349

\section{CIVILIAN APPLICATIONS OF LASER FUSION}

J. Maniscalco, J. Blink, R. Buntzen, J. Hovingh, W. Meier, M. Monsler, and P. Walker

MS. date: November 17, 1977

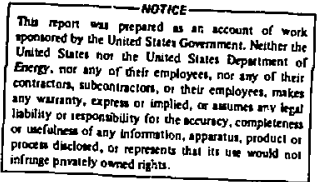




\section{CONTENTS}

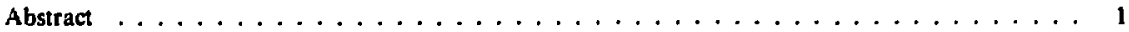

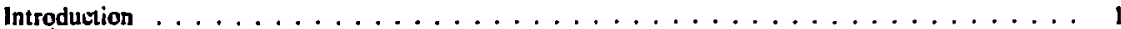

Need For an Inexhaustible Energy Source $\ldots \ldots \ldots \ldots \ldots \ldots \ldots \ldots$

The Payoff $\ldots \ldots \ldots \ldots \ldots \ldots \ldots \ldots \ldots \ldots \ldots$

Fuel For Inertial Confinement Fusion $\ldots \ldots \ldots \ldots \ldots \ldots \ldots$

Long-Range $\operatorname{Plan} \ldots \ldots \ldots \ldots \ldots \ldots \ldots \ldots \ldots \ldots \ldots$

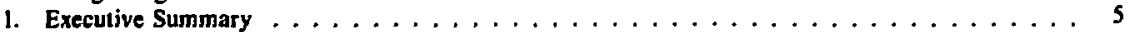

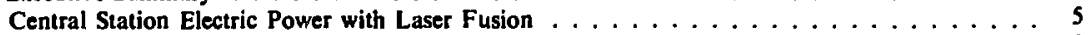

Fusion-Fission Hybrid Reactors $\ldots \ldots \ldots \ldots \ldots \ldots \ldots$

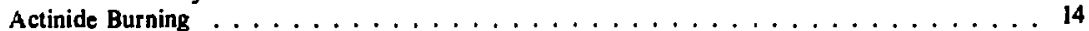

Synthetic Fuel Production $\ldots \ldots \ldots \ldots \ldots \ldots \ldots \ldots$

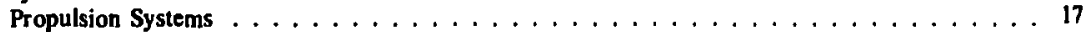

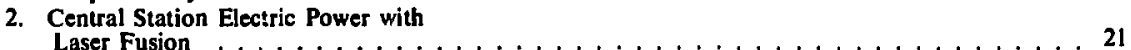

A Comparison of Long-Range Sources

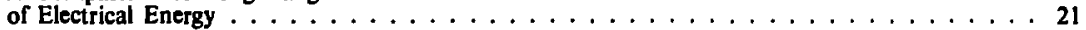

Technical Feasibility of Laser Fusion $\ldots \ldots \ldots \ldots \ldots \ldots \ldots$

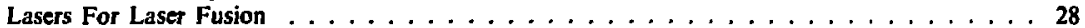

Reactor Concepts For Laser Fusion $\ldots \ldots \ldots \ldots \ldots \ldots$

Preliminary Conceptual Design of a Liquid

Lithium Waterfall Reactor . . . . . . . . . . . . . . . . . . . . 54

3. A Conceptual Design Study for a

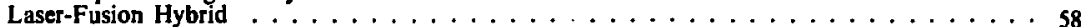

The Laser-Fusion-Hybrid Power-Plant Design . . . . . . . . . . . . . . . . 58

Analysis of the Design . . . . . . . . . . . . . . . . . 64

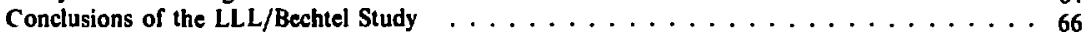

Alternate Approaches Permitting A

Long-Term Fission Economy . . . . . . . . . . . . . . . . . . . . 67

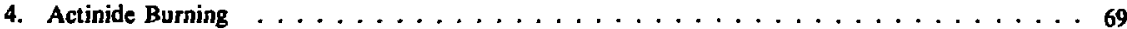

Actinide Burning in Fusion Reactors $\ldots \ldots \ldots \ldots \ldots \ldots \ldots$

Actinide Burning in an Inertially Confined Fusion (ICF) Reactor $\ldots \ldots \ldots \ldots \ldots$

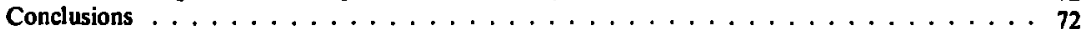

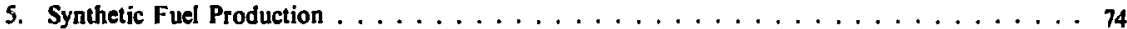

6. Propulsion Systems . . . . . . . . . . . . . . . . . . 79

Engineering Considerations for Fusion Propulsion $\ldots \ldots \ldots \ldots \ldots$

Terrestrial Laser-Fusion Propulsion . . . . . . . . . . . . . . . . . . 80

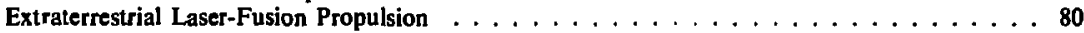

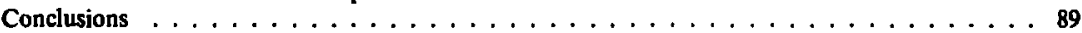

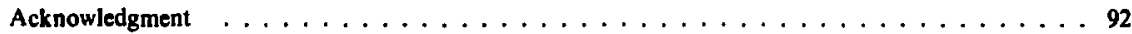

Appendix: A Development Scenario for Inertial Confinement Fusion . . . . . . . . . . 93 


\section{CIVILIAN APPLICATIONS OF LASER FUSION}

\section{ABSTEACT}

We have evaluated the commercial aspects of laser fusion in an attempt to relate the end products (neutrons and energy) to significant commercial applications. We have found that by far the largest markets and highest payoffs for laser fusion are associated with electric power production. Hence, much of this report evaluates the prospects of producing commercial electricity with laser fusion. To this end, we have described in detail a new and promising laser fusion concept-the liquid lithium waterfall reactor. In addition, we have taken the most attractive features from our laser fusion studies and used them to compare laser fusion to other long-range sources of energy (breeder reactors and solar energy). $1+$ is our contention that all three sources of electrical energy should be developed to the point where the final selections are primarily based on economic competitiveness. The other potential applications of laser fusion (fissile fuel production, syrt thetic fuel production, actinide burning, and propulsion) are also discussed, and our preiiminary plan for the engineering development of laser fusion is presented.

\section{INTRODUCTION}

\section{Need For An Inexhaustible Energy Source}

The increasing demand for energy in the United States coupled with a shrinking supply of fossil fuels has led this country to reassess our energy reserves and resources. The results of that reassessment (Figs. 1 and 2) show that our fossil fuels are limited. For example, in 1976, petroleum and natural gas supplied 77\% of the total U. S. energy demand; yet Fig. 2 shows the total $U$. S. resources could not meet that fraction of the demand beyond the turn of the century. All of our exhaustible resources put together (petroleum, natural gas, coal, oil shale, geothermal, and uranium in fission burner reactors) could only meet the demand until somewhere between 2050 and 2225, depending on the demand growth rate. $\mathrm{U}$. S. exhaustible reserses could only meet the demand until about 2040, even in the most optimistic scenario. Thus, it is apparent that "inexhaustible" sources such as fission breeders, solar energy, and fusion reactors are the only sources capable of meeting long-term U. S. energy demand.

Of course, the need to develop long-term sources of energy is not limited to the U.S. Indeed, world energy needs will increase much faster than the
U.S. rate because many third world nations will be greatly increasing their energy comsumption during the next century, although their domestic energy supplies are smaller than ours. Therefore, unless inexhaustible sources are developed, the world energy shortfall will develop sooner than Fig. 2 indicates. The resulting socio-political stresses could lead to wars over dwindling energy sources. To meet our own and the world's energy needs, the U.S. is currently developing inexhaustible sources of energy including solar energy and fusion reactors.

\section{The Payoff}

Electrical energy consumption in the United States has been historically growing at approximately (wice the rate of total energy consumption (7\% per year for the last 25 years). However, a continuation of historical growth is highly unlikely. ERDA's most recent estimate ${ }^{1}$ (April 1977) is that electrical capacity will grow from the present $\mathbf{5 3 0}$ $\mathrm{GW}_{\mathrm{e}}$ to $1390 \mathrm{GW}_{\mathrm{e}}$ in 2000 . These projections represent electrical demand growth rates of $5.3 \%$ from the present to $1985,5 \%$ from 1985 to 1990 , and $4 \%$ from 1990 to 2000 . Even with these reduced growth 


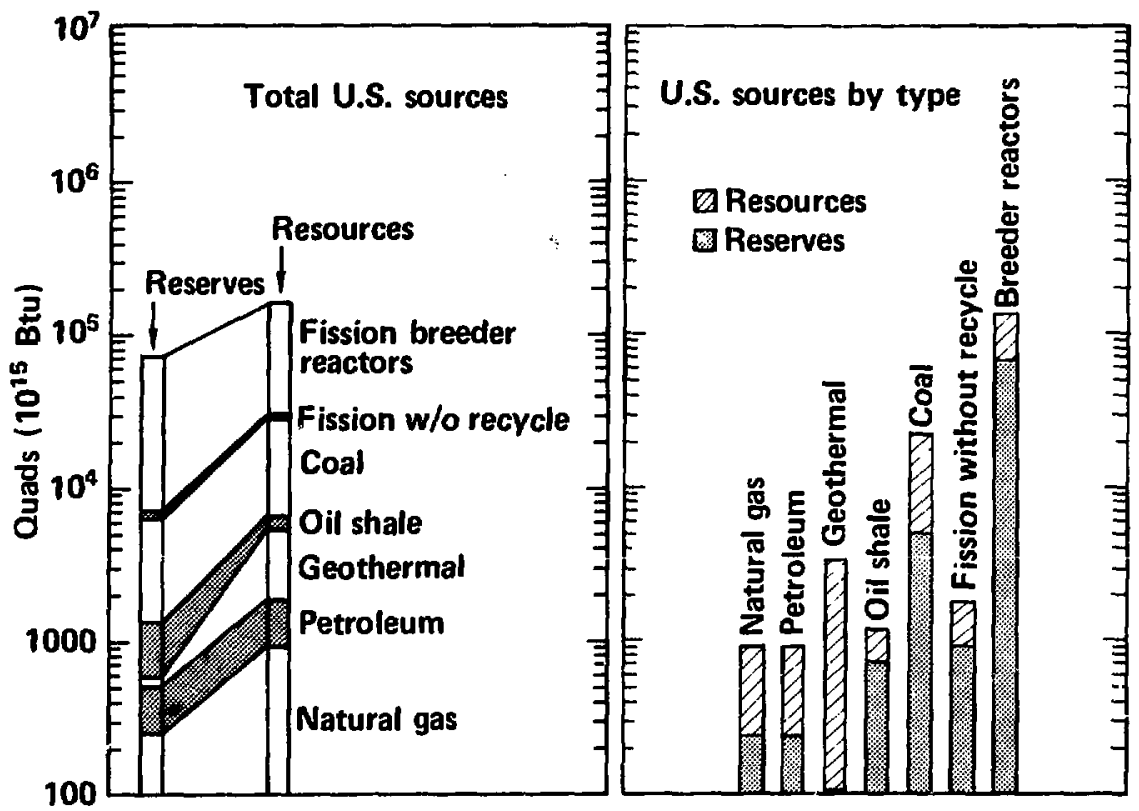

Fig. 1. U. S. exhaubivie energy sources. Reserves refer to an asoured supply of raw materials that can be converted to energy while resnunces refer to raw enterials that may become receverable at highter prices, with more exploration, or with new sechusology.

rates the electricity fraction of our total energy consumption is expected to increase from the present $27 \%$ to $44 \%$ by the end of the century.

In Table I we have extended ERDA's estimate of electrical energy capacity to 2050 . The projections have been made at lower rates of growth in order to provide a lower bound estimate on the number of electrical installations that will be required in the first half of the next century. The significant feature of these projections is that even at zero annual

Table 1. Projection of U.S. electrical capacity from 2000 to 2050.

\begin{tabular}{ccc}
$\begin{array}{c}\text { Annual rate } \\
\text { of growth, } \%\end{array}$ & $\begin{array}{c}\text { Installed } \\
\text { cepteity, OW }\end{array}$ & $\begin{array}{c}\text { Cumulative } \\
\text { number of GW } \\
\text { insullistions }\end{array}$ \\
\hline 3 & 6092 & 8141 \\
1 & 2285 & 3520 \\
0 & 1390 & 2307 \\
\hline
\end{tabular}

Assumes 30-year plent lifotine. growth from 2000 to 2050 , more than 2000 power plants of $\mathrm{GW}_{\mathrm{c}}$ size will have to be constructed. At a cost of a billion dollars per plant, the 2300 plants represent an investment of more than 2 trillion dollars.

In our development scenario, we have estimated that it will cost 10 to 20 billion dollars to develop inertial confinement fusion into a commercially available source of energy. This c st estimate is consistent with projected costs for the development of the fast breeder reactor ( 24 billion) and magnetic fusion energy ( 16 billioit). ${ }^{2}$ The point to be made is that development costs during the next 25 years for long-range sources of electrical energy will be in the tens of billions of dollars, whereas the pay-off to our children in the next century will come in the form of available energy at reasonable cost with savings in the trillions of dollars.

\section{Fuel For Inertial Confinement Fusion}

Early commercial fusion sources of energy will use deuterium-tritium (DT) fuel, in which the 


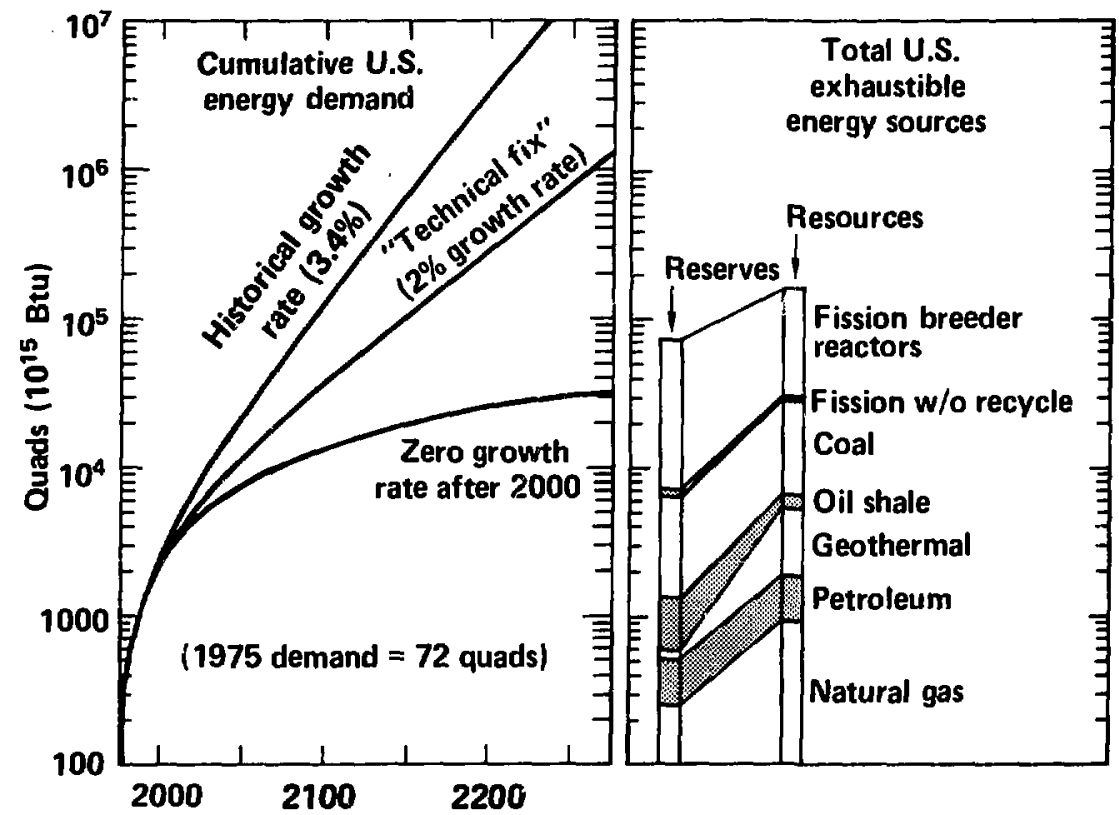

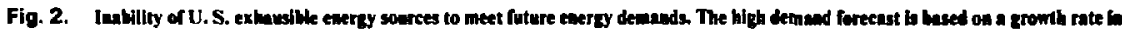

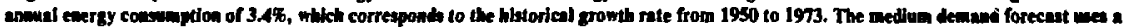

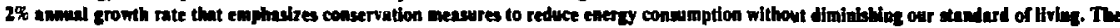
third foreces follows a $2 \%$ growth curve undl the year 2010 widh zero growth therenfter.

tritium is bred from lithium by neutron capture in the blanket. Thus, DT fusion's basic fuels will be deuterium and lithium. Deuterium is present in sea water at a ratio of one part in every 6500 atoms of hydrogen. The oceans and other surface waters on earth contain more than $10^{13}$ tonnes of deuterium. This enormous quantity ensures an almost inexhaustible supply.
Lithium is commercially recovered in the United States from pigmatite and lard-based brines. Present reserves are estimated ${ }^{3}$ to be on the order of $10^{9} \mathrm{~kg}$ and to cost $\$ 20 / \mathrm{kg}$. This supply of lithium would last more than a hundred years in a full fusion economy. Additional resources of lithium recoverable at three times present prices have been estimated to be in excess of $4 \times 10^{9} \mathrm{~kg}$.

Table 2. Inexhaustible resources available from the aceans.

\begin{tabular}{|c|c|c|c|c|c|}
\hline Resource & $\begin{array}{l}\text { Concentration, } \\
\text { g/conne }\end{array}$ & $\begin{array}{c}\text { Therms } \\
\text { energy } \\
\text { per getm, } \\
\mathrm{kWh} / \mathrm{s}\end{array}$ & $\begin{array}{l}\text { Thermal } \\
\text { energy per } \\
\text { tonne of } \\
\text { coawater, } \\
\text { kWh/tonne }\end{array}$ & $\begin{array}{l}\text { Allcwed } \\
\text { cost per } \\
\text { tonse of } \\
\text { wexwater, } \\
\text { s/tome }\end{array}$ & $\begin{array}{l}\text { Enery } \\
\text { avrilible } \\
\text { at } 1976 \\
\text { coasumption } \\
\text { nte, years }\end{array}$ \\
\hline Deuterium & 34 & $261,000^{b}$ & $8,920,000$ & $4460^{\circ}$ & $6 \times 10^{11}$ \\
\hline Lithiom & 0.17 & $83,000^{b}$ & 14,100 & 7 & $9 \times 10^{8}$ \\
\hline Uranium $^{\left.23 s_{U}\right)}$ & 0.0000011 & 24,000 & 0.27 & 0.60014 & $1.7 \times 10^{4}$ \\
\hline
\end{tabular}

Allowable cost for suel is limited to $5 \%$ of the cont of electricity (1.5 millo/kWh).

$b_{\text {Assumes that }} 40 \%$ of the tritium 4 bred in i.i teactions.

Current cost of deuterlum is about $\$ 2$ per tonne of seawater. 
Like deuterium, the lithium resource in the ocean is virtually limitless. Table $\mathbf{2}$ compares the physical and potential economic properties of deuterium, lithium, and uranium extracted from the oceans. If we limit the price for lithium in a fusion reactor to less than $5 \%$ of the presint cost of electricity $(\sim 1.5$ mills/kWh), its value would still be more than $\$ 17,000 / \mathbf{k g}$. This is a price that appears more than adequate to assure the economic feasibility of $\mathrm{cx}$ tracting lithium from the oceans, therebv guaranteeing a virtually inexhaustible source of fuel for fusion.

\section{Long-Range Plan}

This report tries to answer the questions:

- Why do we need laser fusion?

- What are the potential applications of laser fusion?

These questions lead to a third question: How do we take laser fusion from the physics experiments of today to the commercial applications of tomorrow. At LLL, we have initiated a long-range plan (see Ap- pendix) to help answer this question. The plan will guide the Laboratory in its attempts to keep the laser fusion program in line with changes in technical progress, national needs, and the nation's commitment to inertial confinement fusion.

\section{References}

1. No author cited, Discussion Paper on Recent Articles in Science, Energy Research and Development Administration, Division of Magnetic Fusion Energy, Washington. D. C. (1976).

2. No author cited, A National Plan For Energy Research Development and Demonstration: Creating Choices for the Future, Energy Research and Development Administration, Washington, D. C., Rept. ERDA 76-1 (1976).

3. L. G. O'Connell, et al., The Lirhium-Water-Air Battery: A New Concept for Automobile Propulsion, Lawrence Livermore Laboratory, Rept. UCRL-51811 (1975). 


\section{EXECUTIVE SUMMARY}

In our evaluation of the commercial aspects of laser fusion, we have tried to relate the end products (neutrons and energy) to significant commercial applications. Some of the more attractive applications include electric power production, propulsion, hightemperature process heat, fissile fuel production, burnup of radioactive fission waste, and synthetic gas production. In Fig. 1-1 these applications have been separated into two categories: applications that use the fusion reaction for energy or for neutrons.

We have foind that by far the largest markets and highest payoffs for inertial confinement fusion are associated with electric power production. Hence, a major portion of our effort has bien devoted to evaluating the prospects of producing commercial electricity with laser fusion. In our systems studies, we have designed and analyzed hight-average-power laser and reactor concepts that could be used in a laser fusion power plant. The most attractive features resulting from our laser and reactor design studies have been used to compare laser fusion to the other long-range sources of energy (breeder reactors and solar energy). The comparisons have been rade on the basis of abundance, social costs finealth, environment, and safety), technical feasibility, and economic competitiveness. As a resilt of these comparisons. we have found that while laser fusion technology is not as advanced as that of the fast breeder reactor it offers the following advantages over fission:

- No use of wear.ons grade material, thereby avoiding the possibility of diversion for blackmail or sabotage.

- No afterheat cooling problem in case of lossof-coolant accident.

- No nuclear runaway from a reactivity insertion or criticality accident.

- An inexhaustible fuel supply that is available at low cost from the ocean.

- Significantly less radioactive byproducts: with much shorter half-linres.

In comparison to solar electric energy, laser fusion offers the following advantages:

- Significantly reduced cost.

- Geographical and climatic independence.

- Significantly reduced land use.

- No effect on climate.

- More feasible as a base load facility (no energy storage systems required).

- Reduced transmission of electricity.
It is our contention that all three long-range sources of energy should be developed to the point where the final selections are primarily based on economic competitiveness.

Applications other than central station electric power offer the possibility of increasing the overall impact of laser fusion and of hastening its commercial application. Nonelectric applications that we have investigated in this report include fissile fuel production, actinide burning, synthetic fuel production, and propulsion systems. The decision to pursue any of these alternative applications in conjunction with the production of commercial electricity must be made by weighing the recognized benefits against the additional costs for development. In determining recognized bonefits, we have considered:

- The need for the application.

- Alternatives to laser fusion for providing the application.

- The possibility of the application having an earlier impact than central station electric power.

The primary factors favoring early introduction of nonelectric applications are reduced laser fusion performance criteria and relaxed economic constraints.

\section{Central Station Electric Power with Laser Fusion}

\section{Technical Feasibility of \\ Laser Fusion}

Fusion, like all of the advanced sources of electrical energy, is expected to be technically more

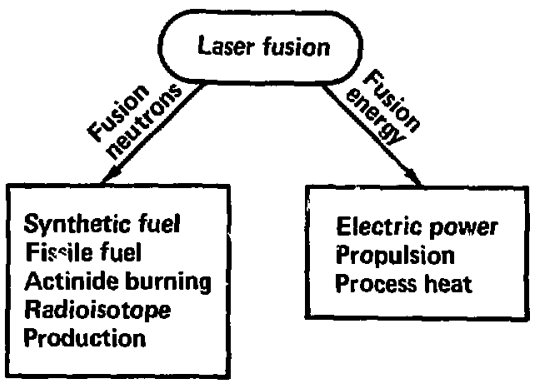

Fig. 1-1. Applications of Laser fusion. 
complex than existing light water reactors (LWRs) or fossil-fueled power plants. The technical feasibility of laser fusion power plan:; requires development of an efficient reactor system that operates reliably at high average power. As an alternative to magnetic confinemeni fusion, laser fusion offers features that could significantly reduce its technical complexity, thereby seducing the amount of advanced technological developinent thit will be required to produce a technically successful fusion power plant. These features include:

- No magnets* (asteisiked items are design dependent)

- Sizing Jexibjlity (i.e., lower powes units)

- Possibility of multiple chambers to provide higher availability

- Flexible geomeíry

- No direct conversion required

- Reduced vacuum pumping requirements* tion*

- Reduced radioactivity îrom neutron acliva-

- Reduced neutron damage to structural materials*

In Chapter 2 we describe a reactor design concept that icatures all of the advantages listed above. The major technical problems associated with a laser fusion power plant that must be solved to realize all of the above advantages include the development of:

- A high-average-power driver with the required efficiency $(>1 \%)$ and reliability $(>70 \%)$.

- A first-wall able to withstand the effects of $x$ rays, debris, and neutrons from the fusion microexplosion.

- Structural materials that can withstand the cumulative damage effects of high-energy neutrons and cyclical stresses.

- Final-focusing elements that can be placed far enough away from the microexplosion or protected so that they can last a reasonable length of time.

- The technology to cheaply mass-produce fusion targets.

Sufficient solutions to the technical problems listed above will allow laser fusion power plants to efficiently produce electricity with high plant availability. The overall efficiency of such a plant can be evaluated through a basic power flow diagram like the one shown in Fig. 1-2. The simple expression shown in this figure gives the plant recirculating power fraction* $\left(\mathrm{P}_{\mathrm{in}} / \mathrm{P}_{\mathrm{g}}\right)$ as a function of laser efficiency $\left(\eta_{L}\right)$, pellet gain $(Q)$, thermal efficiency $\left(\eta_{t}\right)$, neutron energy fraction $\left(f_{n}\right)$, and blanket energy multiplication $\left(\mathrm{M}_{\mathrm{B}}\right)$; i.e.,

\footnotetext{
-Assuming most of the power recirculated is used by the laser.
}

$$
R P F=\frac{P_{\text {in }}}{P_{g}}=\frac{1}{\eta_{L} Q \eta_{i}\left[1+f_{n}\left(M_{B}-1\right)\right]}
$$

Recirculating power fraction is a basic figure-ofmerit for any power plant. For : jerence purposes, fossil-rueled power plants operate with recirculating powers less than $5 \%$ while the majority of present nuclear plants operate at or slightly above this fic'sre. Laser system efficiency $\left(\eta_{\mathrm{L}}\right)$ is a lumped parameter that includes power conditioning, medium rf Jeneration, iasing, and of. ical transport efficiencies. It is defined as the ratio of focusable

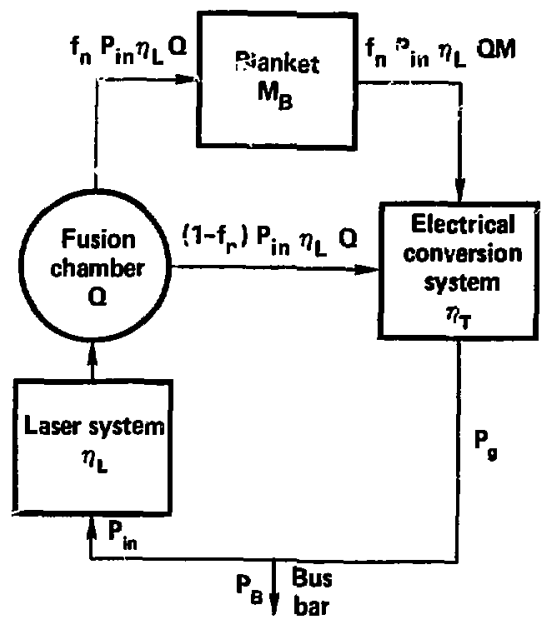

where:

$$
\begin{aligned}
& \eta_{\mathrm{L}}=\text { laser system efficiency } \\
& \mathbf{Q}=\text { pellet gain }=\frac{\text { thermonuclear energy }}{\text { laser light energy }} \\
& \boldsymbol{f}_{\mathbf{n}}=\frac{\text { neutron energy }}{\text { thermonuclear energy }} \\
& M_{\mathrm{B}}=\text { blanket energy multiplication } \\
& \eta_{\boldsymbol{T}}=\text { electrical conversion efficiency } \\
& \mathbf{P}_{\text {in }}=\text { electrical power input to laser } \\
& \mathbf{P}_{\mathbf{g}}=\text { gross electrical power }
\end{aligned}
$$$$
R P F=P_{i n} / P_{g}=1 / \eta_{L} Q_{\eta_{t}}\left(1+f_{n}\left(M_{B}-\eta\right)\right)
$$

Fig. 1-2. Laser fusion power Row diagram. 
beam energy to electrical energy input. Blanket energy multiplication $\left(M_{B}\right)$ is defined as the ratio of blanket thermal energy to fusion neutron energy. The overall system efficiency $\left(\eta_{\mathrm{s}}\right)$ of a laser fusion power plant can be related to the recirculating power fraction (RPF) by the equation;

$$
\eta_{\mathrm{s}}=\eta_{\mathrm{t}}(1-\mathrm{RPF})
$$

From the expressions for recirculating power fraction and system efficiency, it logically follows that the product of laser efficiency $\left(\eta_{L}\right)$ and pellet gain (Q) should provide an excellent figure-of-merit for gauging the prospects of efficiently generating electrical power with laser fusion. This product, which is calied fusion energy gain, represents the ratio of thermonuclear energy produced to electrical energy input to the laser. Fusion energy gain is an extremeiy revealing performance parameter because economic power production with laser fusion will fundamentally lie with the pellet energy gains achieved and the overall efficiencies at which large pulsed ignition sources can operate. In Fig. 1-3 this recirculating power fraction is plotted as a function of fusion energy gain for power plants with a blanket energy multiplication of 1.0 and several thermal efficiencies. Reference designs for laser fusion power plants have used recirculating power fractions in the neighborhood of $25 \%$. However, it may be difficult to compete economically at these high values because in general, plant capital costs scale with the gross electrical power while revenues scale with the neî electrical power.

\section{Cost Comparisons with Breeders and Solar}

Like fission reactors, all of the long range sources of energy will be capital intensive. The cost of electricity from capital intensive power plants is directly proportional to plant cost and inversely proportional to plant capacity factor. We have used this relationship to compare the cost of electricity from laser fusion, breeder reactors, and solar energy. $\dot{A}$ detailed cost analysis of a laser fusion power plant has not been performed. Instead, we have focused on identifying the economic constraints which will ultimately prevail. In line with this objective, we have concentrated on providing estimates of the allowed cost of the laser and reactor portions of an economically attractive power plant. Our results show that a $G W_{e}$ laser fusion power plant can generate electricity at costs that are within $50 \%$ of present prices with lascr and reactor systems whose combined costs are as much as $\mathbf{8 0 0}$ million doliars.

Capital costs for liquid metal fast breeder reactors (LMFBRs) are estimated to be 50 to $75 \%$ more than those of LWRs. Therefore. LMFBRs with capacity factors similar to LWRs (70\%) can be expected to generate electricity at costs that are 20 to $40 \%$ more than present prices. Present estimates indicate that solar power plants will cest two to three times more than LW/Rs presently cost. Moreover, they will operate with capacity factors that are less than $30 \%$ because of the diurnal variation of insolation. As a result solar electric power plants can be expected to generate electricity that costs some six to nine times more than present prices.

From the cost comparisons presented here, it is obvious that the electricity generated by any of the

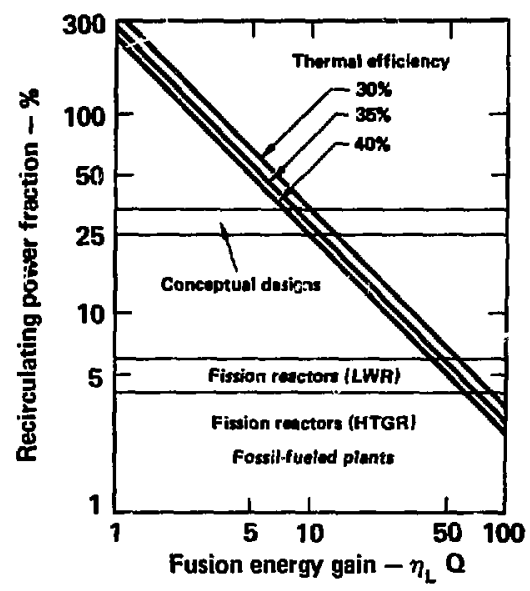

Fig. 1-3. Plant recirculating power requirements as a function of fusion energy gain. A blanket energy multiptication factor of 1.0 was used.

long-range sources of energy will be significantly more expensive than electricity is now. However, within a factor of two, the cost of energy in the U.S. economy is much less critical than assured supply. Present total energy costs are only 5\% of the U.S. gross national product, and it has been estimated that a doubling of energy costs would result in a 2 or $3 \%$ reduction in consumption with little effect on economic growth in the long run.

\section{Lasers for Laser Fusion Power Plants}

The development of a triver for an inertial confinement fusion reactor is technologically challenging. The driver must be capable of taking a small fraction of the power generated by the reactor 
system, form it into pulses of energy at the rate of 1 to 10 per second, concentrate the energy into a pulse a billionth of a second in duration, and project it onto a target a millimeter or so in diameter from a safe distance. Three kinds ol beam generators have the potential to be an inertial confinement fusion driver: jon beims, electron beams, and lasers. Solid state lasers are currently being used for scientific feasibility experiments because they offer the quickest and most cost-effective way of attaining high-gain microexplosions and scientifjc breakeven.

The driver characteristies are determined primarily by target cesign. Target calculations together with economic and system constraints have estab. lished preliminary laser system performance requirements for commercial electric power production. The nominal requirements include

$\begin{array}{ll}\text { - Wavelength } & 250-2000 \mathrm{~nm} \\ \text { - Pulsc duration } & 1-10 \mathrm{~ns} \\ \text { - Pulse energy } & 300-3000 \mathrm{~kJ} \\ \text { - Pulsc power } & >200 \mathrm{TW} \\ \text { - Pulse repetition rate } & 1.10 \mathrm{IJ} \\ \text { - Average pow:- } & 1-10 \mathrm{MW} \\ \text { - Overall efficiency } & >1 \%\end{array}$

These requirements continue to evolve, reflecting new knowledge in larget and laser physics, materials and laser enginecring, systems analysis, and cconomics.

There is no laser that currently satisfics all these requirements. Design of such a laser should be based on two aspects: efficiency and scalability. $\wedge$ laser system of high efficiency will have lower recirculating power needs and lower capital equipment costs. The efficiency depends on the losses due (o) power conditioning, fluoreseence production. coupling to the laser molecule, optical extraction, and pumping, cooling, and chemical regeneration of the flowing gases.

Scalability to large size is important because a laser built with a large single-aperture size and high energy per pulse lowers costs by reducing the number of beams and components. The stalability depends on optical and e-beam foil damage limits, pump constraints, flow and gas uniformity constraints, parasitic suppression, thermal disturtion, and arrival at a configuration that simultaneously pushes all the important technology barriers.

In the search for a laser that can meet the above requirement, several laser media appear to be promising. Depending on the medium, three different kinds of stimulated emission can occur:

Electric dipole allowed transitions
- Electric dipole forbidden transitions (e.g., magnetic dipole or electric quadruprile)

- Opticully induced transitions (e.g., Raman scattcring, frequency tripling, etc.)

lexamples of laser media that fall into the first catcgory include rare gas and rare gas halogen excimers." Laser media of the second type include iodine, the Group VI clements, and the rare earth wapors. The third type includes processes such as electronic Raman scattering in $\mathrm{Hg}_{2}^{*}$ or backward wave Raman scattering. In Chapter 2 , we present a preliminary evaluation of the efficiency and scalability of photolytically pumped Group VI atomic lasers. In Table 1-1, we list features of photolytic lasers that recommend them for fusion power plants. These features include favorable target coupling characteristics, high-energy storage per aperlure, promising overall efficiency, and a choice of lasing atom and pemp molecule combir'stions.

Table 1-2 presents the major results of the evaluation. The maximum overall system efficiency and the maximum overall stored energy for a single amplifier module is given for the specific case of a fluoreseer-pumped windowless configuration.

These sulculations emphasize obtaining maximum size; higher efficiencies would result even for the same configuration, if we trade off scalability for efficiency. $\Lambda$ mplifiers can conceivably be built large enough to reach $1 \mathrm{MJ}$ in a reasonable number of beams. Obtaining adequate efficiency will be the most difficult task; only the setenium system promises an overall efficiency above $1 \%$, and even then this value is at the high end of a range of estimated efficiencies. The iodine system requires a tenfold higher power input than the selenium system. This could only be accomplished by operating at 10 times the recirculating power or 10 times the fusion pellet gain, circumstances that are either ceonomicaily or physically unikely.

This evaluation has been instrumental in showing that a fluorescence-pumped photolytic laser has the potentia, to be a full size fusion driver. There are significant uncertainties remaining, and much experimental work on kinetics, gain me!surements, self absorption. bleaching wave dy.amics and beam quality remains to be done. However, if the experiments now being undertaken bear fruit, fusion baser amplifiers of significant size $\left(\geqslant 10^{5} \mathrm{~J}\right)$ and adequate efficiency are possible.

\footnotetext{
An extiner is a molucule which only exists in the excited stale: e.g. $X_{2}$, where the asterisk indicates the molecule is in the extitited sistte.
} 
Table 1-1. Features of photolytic lasers.

I. Choice of system:

\begin{tabular}{|c|c|c|c|c|}
\hline $\begin{array}{l}\text { Lasing } \\
\text { atom }\end{array}$ & $\begin{array}{c}\text { Lasing } \\
\text { wavelength, nm }\end{array}$ & $\begin{array}{c}\text { Parent } \\
\text { molectie }\end{array}$ & $\begin{array}{c}\text { filuoreseer } \\
\text { excimer }\end{array}$ & $\begin{array}{c}\begin{array}{c}\text { Pfuorescer } \\
\text { wayelenglh, nm }\end{array} \\
\text {. }\end{array}$ \\
\hline Iodine & 1130 & $C_{3} F_{7} I$ & XeBr * & 280 \\
\hline Oxygen & 558 & $\mathrm{~N}_{2}^{3} \mathrm{O}$ & $\Lambda r_{2}^{*}$ & 130 \\
\hline Sulfur & 772 & cos & $\mathrm{Kr}_{2}^{*}$ & 150 \\
\hline Selenium & 489 & $\cos \mathrm{e}$ & $x_{2}^{*}$ & 170 \\
\hline
\end{tabular}

2. Favorable target coupling characteristics:

- Wavejength: $\lambda=0.5-1.3 \mu \mathrm{m}$

- Slıort pulse duration: $r \sim 1-10 \mathrm{~ns}$

3. High-energy storage:

- $F_{\text {saturatimon }} \sim 1-10 \mathrm{~J} / \mathrm{cmi}^{2}$

4. Iavorable scaling characteristick:

- Configtrations allow nowing, high-average-puwer systens.

- Can use low-pressurc ( 1 atm), low-temperature $(\sim 300 \mathrm{~K})$, low-index gases (e.g., heliun) for geod beam qual:ty.

- Beam quality and gain uniformity limited by gas density uniformity, not hy e-beam deposition uniformi $j$.

- Aperture scaling limited by materials and gain constraints rather than by properies of the atonis and molecules.

5. Efficiency $21 \%$ possible from the physies.

Table 1-2. Efficiency and scalability of photolytically pumped Group VI tasers.

\begin{tabular}{lllll}
\hline & Oxygen & Sulfur & Selenium & Iodine \\
\hline Maximum amplifier size & $350 \mathrm{~kJ}$ & $130 \mathrm{~kJ}$ & $210 \mathrm{~kJ}$ & $140 \mathrm{~kJ}$ \\
Overall Jaser system efficiency & $0.33 \%$ & $0.72 \%$ & $1.53 \%$ & $0.14 \%$ \\
\hline
\end{tabular}

The crucial issue is to improve the overall efficieney-borh the electrical and flow efficiencies. The best chance of doing this involves improving the coupling of the pump radiation to the laser species, a problem amenable to some innovative thought. Excimer laser pumping is one possibility-better directionality of the pump beam will help immensely, although excimer laser efficiencies are less than excimer fluorescer efficiencies for all the rare gases. According!y, LLL has developed several ideas for pumping geometrics that use both sides of the slab of fluorescer gas, a stratagem which can raise both the electrical efficiency and the flow efficiency by nearly a factor of two. This would put the selenium laser in the 7 管 electrical efficiency range (3-4\%, overa!l efficiency range), making it an attractive prospect for a commercial rower plant driver.

\section{An Inertial Confinement Fusion Reactor Concept}

Approximately $32 \%$ of the energy from a DT thermonuclear microexplosion is in the form of $x$ rays and energetic debris. This energy is deposited over a very short range in the first wall cuusing high temperiures, ablation, and cyclical stresses. Several different approaches to the first wall problem have been considered jncluding wet wall, magnetically protected wall, dry wall, and fluid wall concepts. These approuches differ primarily in the way the inner surface of the vacuum chamber interacts with 
the $x$ rays and microexplosion debris. The wet wall concept features a thin layer of liquid metal over a metal wall. This layer protects the wall from the blistering and ablation that would normally occur. In the magnetically protected wall approach, a solenoid diverts the pellet debris from the radial portion of a cylindrical blanket to conical collectors at the top and bottom. In the dry wall approach, a sacrificial metallic or ceramic liner is placed be. tween the fusion thamber and the blanket.

The fuid wall concept is a new and promising approach which has been developed in our scoping studies. In this approach, the first structural wall is shiclded from $\times$ rays, neutrons, plasma, and shock effects by a thick falling region of lithium in liquid or solid pellet form. The fali contains enough moderating material to degrade the fusion neutron spectrum to the point where neutron damage levels in structural materials will be low. These lower levcls ullow consideration of smaller blanket structures which could last for the useful lifetime of the plant.

The liquid lithium waterfall reactor is shown in Fig. 1-4. The principal feature of this design is a thick continuously recyclable first wall of lithium. On each shot the waterfall is disassembled; between each shot it is reestablished. The lithium is continuously pumped to the top of the vacuum chamber through a reservoir region which separates the first wall from the pressure vessel. A small fraction of the lithium in the fall and resevoir regions is circulated to the heat exchanger which in turn transfers heat to the stearr, generation cycle. Liquid lithium serves as the primary coolant, the neutron moderator, and the fertile material for tritium brceding. It also protects the structural wall.

Our calculations show that a $60-\mathrm{cm}$ thick region of falling liquid lithium can be maintained with less ahan 5\% of the plants' output power. The fusion neutrons deposit the majority of their energy in the falling lithium region, thereby mitigating the problems associated with neutron damage and activation of the structure. For example, our neutronics calculations show that the $60 \mathrm{~cm}$ region allenuates the fusion newisun flux to a point where the blanket structure can last the lifetime of the plant (30 yrs).

As one might suspect, the liquid lithium waterfall concept has excellent tritium breeding characteristics and inherently good cooling characteristics. With no structural material between the fusion neutrons and the lithium fall, the design takes full advantage of the lithium tritium breeding reactions. In fact, this reactor concept will produce enough excess tritium ( $>1.5$ ) to relieve other applications such as propulsion and synthetic gas production of the burden of breeding and recovering their own tritsum.

L.jthium is compatible with stainless steel to temperatures approaching $800 \mathrm{~K}$ : this compatibility allows thermal to electrical conversion efficiencies approaching $40 \%$. With an operating temperature in the neighborhood of $750 \mathrm{~K}$, the vapor pressure of lithium is sufficiently low $\left(-10^{-3}\right.$ Torr) to maintain adequate vacuum conditions in the laser fusion chamber. In fact, the lithium waterfall will itself act as a vacuum pump by condensing the lithiuin that is vaporized from the microexplosion.

Chapter 2 pressents a preliminary conceptual design of a laser fusion power plant that incorporates the liquid lithium waterfall reactor concept. After selecting a representative set of laser and target parameters, we found that the plant could produce $1160 \mathrm{MW}$ of thermal power. The thermal energy is converted to eleetrical energy with an efficiency of $38 \%$. Of the total $440 \mathrm{MW}_{c}$ produced. $80 \mathrm{MW}$ of electrical power is recirculated for plant operation (lasers and lithium flow). This results in a power plant with a net electrical production of 360 $M W_{c}$ and an overall system efficiency of $3 ! \%$.

\section{Fusion-Fission Hybrid Reactors}

Fusion-fission hybrid reactors can be viewed as subcritical fission blankets. They are driven and controlled by an internal source of high energy neutrons, the fusion reactor. The hybrid reactor combines naturally the power-richness of fission systems with the fast-neutron-richness of fusion power sources. The term fast-neutron-rich should be emphasized because it is the $14.1-\mathrm{MeV}$ neutrons from DT-fusion reactions that provide the reactor with the ability to generate substantial amounts of power and fissile fuels from the abundant fertile materials: ${ }^{232} \mathrm{Th}$ and ${ }^{238} \mathrm{U}$. Since the hybrid reactor operates in a subcritical mode with a low fissilematerial inventory, there is an absolute guarantee against a nuclear excursion.

For several years, we have been investigating potential of producing fissile fuel and electricity in a laser-fusion-driven subcritical fission blanket (i.e., a hybrid reactor). Our earlier studies ${ }^{1-3}$ primarily used neutronic methods of analysis to identify attractive systems and provide an upper-bound estimate of performance. They also demonstrated that fusion-fission hybrids could be designed to meet a broad spectrum of fissile-fuel-producing and energy-multiplying requirements. The following are 


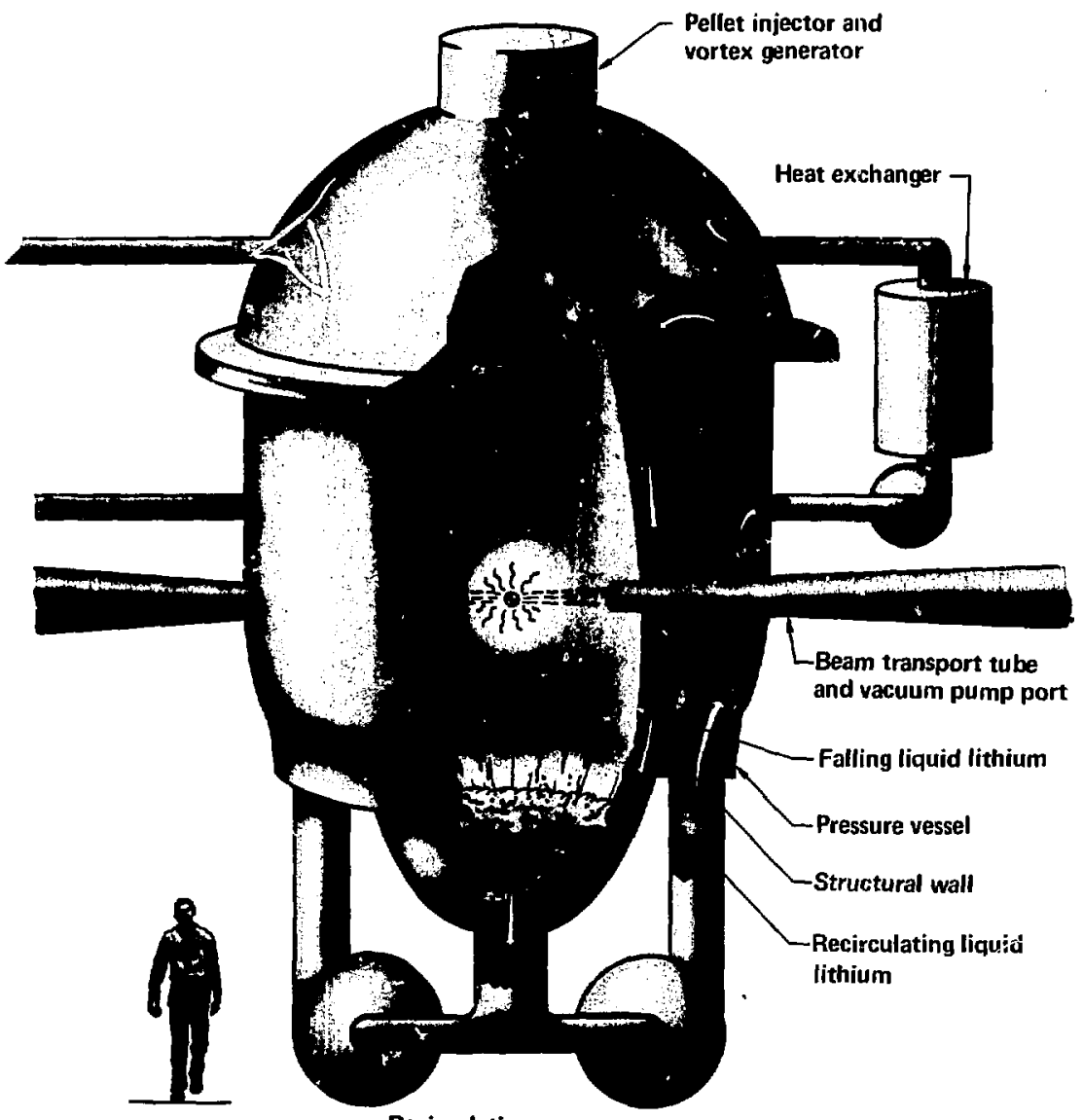

Recirculation pumps

Fig. 1-4. Liquld lithium waterfall concept.

the three most significant features that emerged from our neutronic scoping studies:

1. Laser fusion hybrids produce 10 times more fissile fuel (per unit of thermal energy generated) than fission breeder reactors.

2. Laser-fusion hybrids ecomonically produce electricity with much lower laser efficiencies and pellet gains than those required for pure fusion.
3. Inertially confined fusion systems can more easily accommodate a fission blanket than magnetically confined systems.

The energy-multiplication characteristies of all the hybrid blankets we have neutronically analyzed are summarized in Fig. 1-5. As the figure dramatically points out, much less fusio anergy gain is required if the fission blanket is colistructed 
of fissile material with a high energy-multiplication capability $\left(M_{B}\right)$. For example, in an energy breakeven mode, the required fusion energy gain for a depleted uranium-fueled hybrid is 2 to 10 times less than the fusion energy gain required for a thorium fueled hybrid.

Figure I-6 shows the production cap bilities of laser-driven hybrids and fission breeder reactors along with the consumption requirements of thermal burner and converter reactors. (Reactors are considered "burners" for values of $C<0.8$, converters for $0.8<\mathrm{C}<1.0$, and breeders for $C \geqslant 1.0$ where $C$, the conversion ratio, is defined as the ratio of fissile atoms produced to fissile atoms destroyed,) In Fig. 1-5, we saw that depleted uranium-fueled hybrids can operate with much lower fusion energy gains than those required for thorium blankets. Figure 1-6 shows that thoriumfueled hybrids outperform uranium-fueled hybrids on the basis of fissile fuel produced per unit of thermal energy.

It is interesting to match the consumption requirements of the fission reactors to the production capabilities of the breeders. $A$ thorium fast-fission hybrid provides enough fissile material to fuel 20 high-temperature gas-cooled reactors (HTGRs) of equivalent thermal power while a depieted uraniamfueled hybrid produces enough fissile material to fuel seven light water react ors (LWRs) of equivalent thermal power. Conversely, if fast breeder reactors (FBRs) were used to fuel LWRs, they would not produce enough excess fissile material to fuel even one LWR of equivalent thermal power."

Our neutronic results were encouraging, bul it was apparent that a more accurate assessement of the hybrid's potential and a definitive ranking of the more promising concepts would also require studies of the engineering safety and economic issues. With this in mind, Bechtel Corporation was engaged to assist us in a conceptual design study of a laser fusion hybrid. The design that evolved is a depletedu ranium-fueled, fast-fission blanket which produces fissile plutonium and electricity. The fission blanket surrounding the fusion chamber was designed with current fission technology so as to simplify the assessment of the hybrid system's technical feasibility.

-The excess fissile material produced in an FBR is best used to fuel a new FBR.

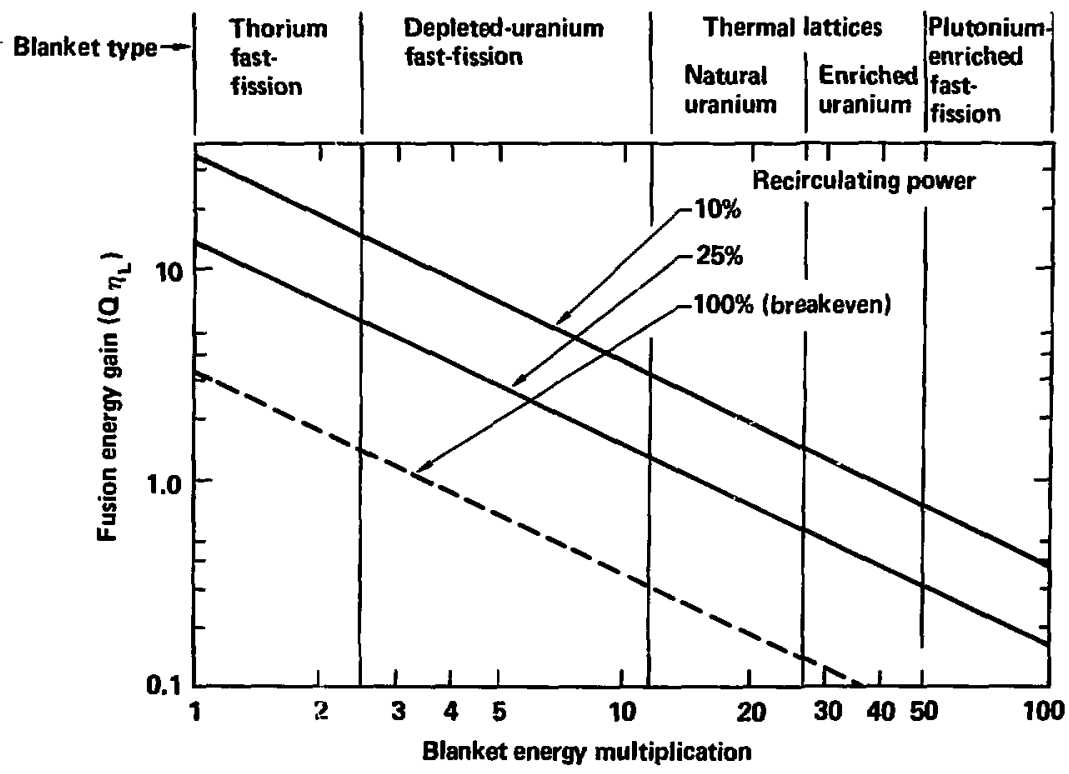

Fig. 1-5. Laser fusion energy goin requirements for hybrid fusion-fission systems. 


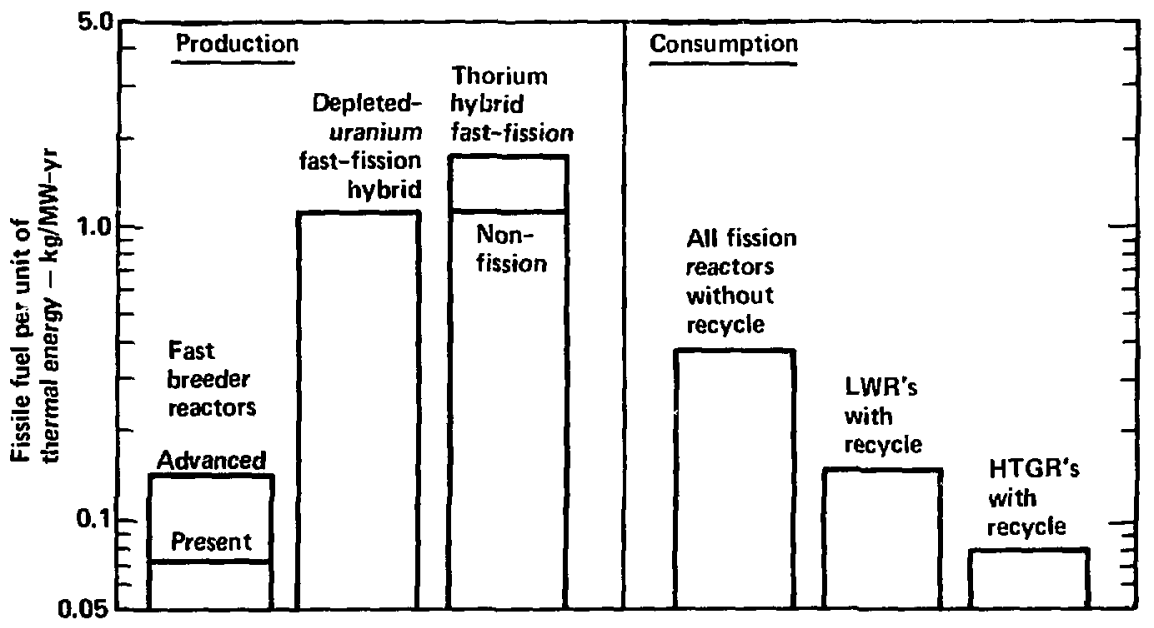

Fig. 1-6. The production and consumption of fissile fuel.

One of the study's objectives was to determine if fissile fuel could be produced with laser fusion. This part of the study consisted of analyzing the integrated engineering performance of the conceptual design and identifying the required laser/pellet performance. The performance of the laser fusion hybrid was also compared to a typical fast breeder reactor. The results from the conceptual design study substantiate the previously given upperbound neutronic results. They show that the laser fusion hybrid produces enough fissile material to fuel more than six light water reactors (LW'Rs) of equivalent thermal power while operating in a regime which requires an order of magnitude less laser and pellet performance than pure laser fusion. In comparison to a fast breeder reactor, the hybrid produces 10 times more fissile fuel.

We also found that the hybrid will be two to three times more expensive than a light water reactor. The cost estimate varies primarily because of the uncertainties in the design of the pellet production facility and the laser system. However, the cost of electricity is more important than the hybrid's capital cost in a scenario in which hybrids provide fuel for LWRs. In Fig. 1-7, the cost of electricity has been plotted as a function of the cost of LWR fissile fuel for hybrids with varying capital costs. The results indicate that the cost of electricity is insensitive to the capital cost of the laser fusion hybrid. Specifically, the cost of electricity increases by only 20 to $40 \%$ when the capital cost of the hybrid ranges from two to three times more than the LWR.

Light water reactors will be the major (and most likely the only) source of commercial nuclear electric power for the remainder of this century. Their dominance over coal fired plants as base-load electrical generators will be strongly dependent on the

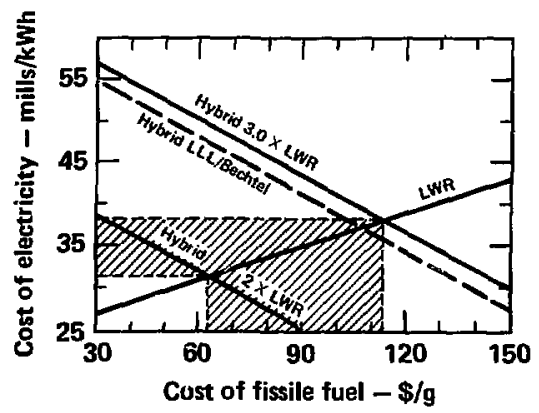

Fig. 1-7. Cost of fissile fuel and electricity from a hybrid. The intersection points of the curves determine the cost of electricity and fissile fuel in the hybrid-LWR scenario. For example, the cost of electricity increnses by only 20 to $40 \%$ when the capital cost of the bybrid manges inom two to three times mare than the LWR. 
adequacy of their long term fissile fuel supply. By converting the ${ }^{238} \mathrm{U}$ in uraniun to fissile plutonium the hybrid could extend the fissile fuel supply for economically proven LWRs by two orders of magnitude. Fast breeder reactors also offer the prospect of more fully utilizing the uranium resources; however, they will not provide fissile tuel for LWRs. Therefore, the usefulness of fast breeder reactors will be entirely dependent on their economic competitiveness as power plants.

When we analyzed the cost of a laser fusion hybrid, we found that it was two to three times more expensive than a light water reactor. The variance in the cost estimate is primarily due to the uncertainties in the design of the target production facility and the laser system. However, the major issue concerning a laser fusion hybrid is not how much it will cost but rather how much electricity will cost if hybrids provide fuel for burner reactors. In Fig. 1.7, the cost of electricity has been plotted as a function of fissile fuel cost and hybrid capital cost. These results indicate that the cost of electricity is insensitive to the capital cost of the laser fusion hybrid.

\section{Actinide Burning}

The neutrons from DT fusion systems can be used to transmute or change the long lived waste products from fission reactors (primarily actinides) to short lived or stable isotopes. This method has been proposed as an alternative to geological storage for the long lived waste. Since actinides have half lives that are long compared to geological events such as ice ages and earthquake fault movement, the acceptablility of a geographical disposal concept is debatable.

If all the waste products from fission reactors are allowed to decay, the actinide waste will comprise most of the radiological hazard after 400 years. Thus, if the actinides can be transmuted into stable isotopes or fissioned into relatively short lived fission products, the duration of the radioactive hazard can be reduced substantially. Several studies haye investigated the possibility of recycling actinide waste in thermal fission reactors and fast breeder reactors. These studies have shown that actinide burning in fission reactors is feasible and most efficient in the harder neutron spec $a$ of fast breeder reactors. It logically follows that fastneutron-rich fusion systems could even more effectively serve the same role.

Two studies of actinide burning in fusion reactors have been completed. Battelle Northwest Laboratory (BNWL) performed a study of actinide burning in a beryllium blanket with a first wall fusion neutron flux of $10 \mathrm{MW} / \mathrm{m}^{2}$ and an irradiation time of $\mathbf{5 0 0}$ days. They concluded that the first wall Joading was an order of magnitude too smal! for effective transmutation in the moderated niutron spectrum of the beryllium blanket. The Fusion Power Division of Westinghouse (WFPS) has recently completed an investigation of actinide burning in a fast spectrum. The irradiation time in the study was 30 years with first wall neutron fluxes of I.15, 5, and $10 \mathrm{MW} / \mathrm{m}^{2}$ in a Tokamak configuration. The WFPS reactor blanket produced 13,000 $\mathrm{MW}_{1}$ of power. Their results for the $1.15 \mathrm{MW} / \mathrm{m}^{2}$ case were similar to that of the BNW! study, which shows a hazard index no better than that of natural decay of the waste in the $10^{5}$ year period. However, for the $10 \mathrm{MW} / \mathrm{m}^{2}$ case, the hazard index ci the waste is reduced about three orders of magnitude. A comparison of the relative ingestion hazards from actinides as a function of decay time for various fusion neutron first wall fluxes following 30 years of irradiation is shown in Fig. 1-8. Note that the injection hazards of the unirradiated actinides are reduced to those of the parent ore within 100 years and become increasingly less hazardous with time thereafter." These results clearly demonstrate that for effective burning of actinide waste the product of irradiation time and first wall flux must be on the order of $100 \mathrm{MW}$-year $/ \mathrm{m}^{2}$.

\footnotetext{
-The actinide hazard is concentrated rather that diluted in as large volume like the parent ore.
}

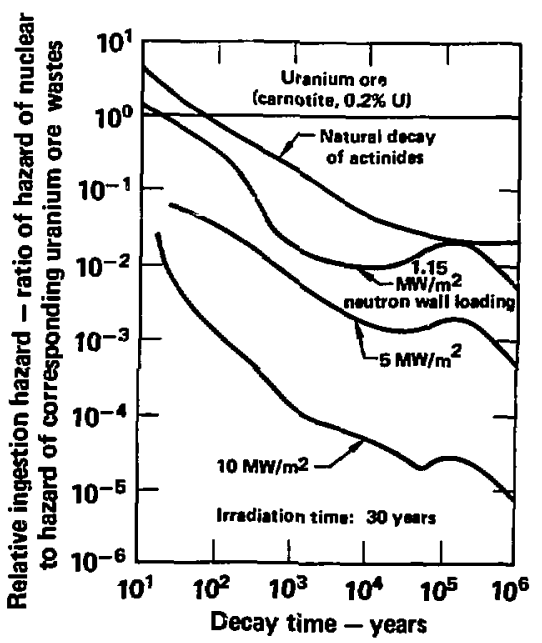

Fig. 1-8. Comparison of relative ingestion hazands from actinides as functions of decay tine. 
Because inertially confined fusion systenıs can operate at higher first wall loadings and more easily accommodate fissioning blankets, they will be more effective actinide burners than magnetically confined fusion systems. An alternative scheme for burning actinide waste in an inertially confined fusion system is to incorporate the actinide waste into the fusion target. This option has the advantage of increasing the neutron fluence per shot by more than nine orders of magnitude. Calculations have shown that most of the actinide muterial seeded into the target can be burned. The disadvantages of burning the actinides in the target are that pellet fabrication and processing of fission product waste becomes more complicated than burning the aclinides in a blanket and that the actinide mass burn rate is very small due to limits on the amount that can be compressed.

It would appear that the inertially confined fusion reactor would be more attractive for actinide burning applications than alternative systems (LWR, LMFBR, and magnetically confined fusion). However. the actinide burning process is of questionable desirability for the following reasons:

High-level wastes would be handle' ,1ore often than they would be if all fission re.ctor waste were immediately encapsulated and stored.

- The short-term hazards increase during early irradiation as a result of the production of ad. ditional actinides from neutron capture.

Applications for actinides exist.

- Reduction of effective half-life from the natural half-life for actinides is of questionable value. Even without irradiation, the actinide ingestion hazard decays to the hazard of the parent ore after a relatively short storage period (100 years).

If society deems the long-term radiological hazards from fission reaclor actinides high enough to merit construction of actinide burners, then inertially confined fusion technology could best perform the required irradiation.

\section{Synthetic Fuel Production}

Our oil and natural gas supplies ase declining rapidly. Hence, the ability to produce large quantities of portable chemical fuels for our homes, industries, and vehicles will be a necessity within the next 100 years. Natural gas has been the preferred fuel for residential heating and industrial process heat because it is environmentally acceptable, easy to use, and, up until recently, inexpensive and reliable. Depletion of natural reserves has given added emphasis to the search for an alternative fuel, and the potential role of hydrogen has been discussed in this respect, both as a fuel itself and as an intermediate product used in the production of synthetic fuels.

Hydrogen production processes can be classified on the basis of the form of primary energy used: thermochemical. electrolytic, or radiolytic. Hydrogen production by radiolysis can be achieved in several schemes which require an intense source of radiation and generally employ water as the starting material because of its abundance. The penetrating neutron radiation of an inertial confinement fusion reactor could be used for radiolysis by incorporating sufficient quantities of $\mathrm{H}_{2} \mathrm{O}$ in the blanket regions surrounding the fusion vacuum chamber. More than 25\% of the energy released by the fusion microexplosion is carried by the pellet debris, alpha particles and $x$ rays. This energy is deposited in the vacuum chamber wall and thus would not be available for radiolytic decomposition.

A concept often used in the discussion of radiation-induced chemical processes is the $G$ value. The $G$ value for the formation of a chemical species is defined as the number of molecules produced per $100 \mathrm{eV}$ of energ: desposited in the production regions by incoming radiation. The generally accepted $\mathrm{G}$ value for $\mathrm{H}_{2}$ from water is 0.45 while $\mathrm{G}$ values as high as 13.6 have been measured in experiments using pure steam exposed to high-energy neutrons. The conversion efficiency of nuclear energy deposited in the hydrogen producing regions to usable chemical energy is equal to $0.01 \mathrm{Gh}$, where $h$ is the heat of combustion of $\mathrm{H}_{2}(2.5 \mathrm{eV})$. Thus for a $G$ factor of 13.6, 34\% of the energy deposited in the steam is converted to the potential combustion energy of $\mathrm{H}_{2}$, while $66 \%$ of it appears as the thermal energy of the steam.

Note that only a fraction of the available neutron energy would actually be deposited in the steam blankets; the remainder would be deposited in reactor structurai materiais and, ihisis, wouid be unavailable for radiolysis of $\mathrm{H}_{2} \mathrm{O}$. The system efficiency for hydrogen power production, defined as the ratio of usable chemical energy produced to the energy deposited in all regions of the reactor, is on the arder of $15 \%$ for the $G=13.6$ steam system.

The hydrogen production efficiencies presented here can be put into perspective by comparing them to thermochemical processes which produce synthetic fuels with efficiencies ranging from $30 \%$ to over $60 \%$, and electrolysis of water which produces hydrogen with efficiencies of 50-100\%. Assuming a $40 \%$ thermal-to-electric conversion efficiency, the electrolysis process produces hydrogen with an overall efficiency up to $40 \%$. An interesting alternative to the simple radiolysis process would be to convert the thermal energy deposited in the system 
to electricity and use the electricity to produce hydrogen by electrolysis (radiolysis/electrolysis hybrid). Figure $1-9$ compares the various conversion processes on the basis of recoverable energy per unit of thermal energy availatile.

Current costs of natural gas supplies are in the area of $\$ 1 /$ million Btu but may climb to $\$ 3 /$ million Btu before the end of the century. The present estimated cost of syythetic gas from coal gasification plants ranges from about $\$ 4.50 /$ million Btu for the current process to as low as $\$ 3.50 /$ million Btu for future plants. In siru coal gasification may be able to produce gas at a cost of about $\$ 2 /$ million Btu.

The cost of hydrogen produced by the various laser fusion driven processes is, at minimum, equal to the cost of energy from the fusion reactor divided by the overall energy conversion efficiency. For the sake of providing a lower bound estimate of cost, assume that a laser fusion power plant can produce electricity at the same price as an LWR $(\sim 25$ mills/kWh). Table 1-3 gives the source energy cost of producing hydrogen by the various processes. These values reflect only the cost of using the laser fusion reactor as the source of energy for the various processes. Additional costs will vary for the different processes depending on the cost of the components required. Large electrolysis units, for example, will add about $\$ 1 /$ rnillion Btu to the cost of hydrogen. It should also be noted that the cost of cource energy for the processes that do not require electrical energy have been reduced from $\$ 2.93$ to $\$ 2.46 /$ million Btu because the electrical power generating equipment (turbine generators and turbine buildings) accounts for $20 \%$ of the capital cost of an LWR and the total capital cust is about $80 \%$ of the price of electricity.

It is clear that there is no economic incentive for the production of synthetic fuels with laser fusion at the current price of natural gas nor at the projected cost of gas from coal gasification. Even so, such a system may eventually be required. Oil and natural gas currently account for about $75 \%$ of this nation"s energy consumption. As these fuels are depleted, the ubility to produce large quantities of portablc fuel will become a necessity. Laser fusion driven hydrogen production processes represent a virtually inexhaustible supply of portable fuel.

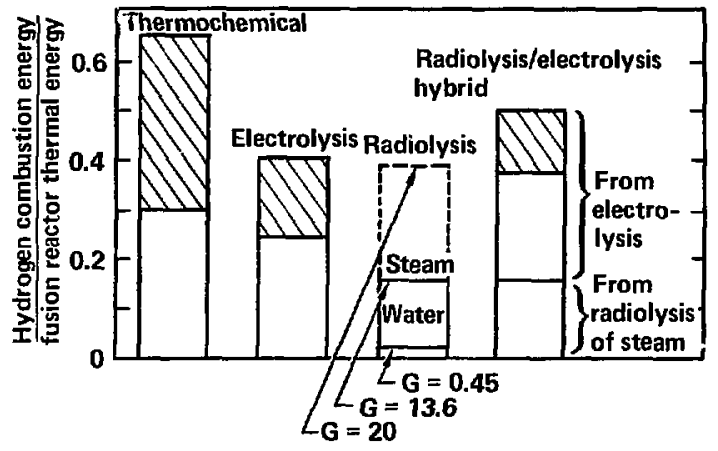

Fig. 1-9. Cumbustion energy of hydrogen recuterable per unit of fusion reactor thermal energy. The fraction of the neutron energy deposited in hydrogen processing repions is Renerously assuntied to be $100 \%$ for radiolysis of water and $60 \%$ for rodiolysis of steam. It is milkely that a dedicated radiolysis hydrogen production facility could ever prove practical unless a liquid medium with a higher $\boldsymbol{C}$, value is found. Therefore, a hypothetical $C=20$ liguid medium system is shown.

Table 1-3. Energy source component of the cost of hydrogen.

\begin{tabular}{|c|c|c|c|}
\hline & $\begin{array}{l}\text { Cost of source } \\
\text { energy, s/MBtu }\end{array}$ & $\begin{array}{c}\text { Energy conversion } \\
\text { effriciency }\end{array}$ & $\begin{array}{l}\text { Energy source } \\
\text { component of } \\
\text { hydrogen cost, } \\
\text { //MBtu }\end{array}$ \\
\hline Thermochemical & 2.46 & $0.30-0.65$ & $3.80-8.20$ \\
\hline Electrolysis & 2.93 & $0.24-0.40$ & $7.30-12.20$ \\
\hline Radiolysis & 2.46 & $0.16-0.38^{b}$ & $6.50-15.40$ \\
\hline Radiolysis/electrolysis & 2.93 & $0.36-0.50$ & $5.90-8.10$ \\
\hline
\end{tabular}

${ }^{a}$ Does not include cost of chemical processing plant, electrolysis units or radiolysis components required.

$b_{0.16}$ and 0.38 correspond to $G$ values of 13.6 and 20 , respectively. 


\section{Propulsion Systems}

Propulsion systems powered by laser fusion reactors could be used to drive large aircraft, ships, and extraterrestrial craft. Such systems could be in. stalled in military vehicles befire laser fusion electric power plants become a reality because of relaxed economic constraints. However, this assumes that adeauate supplies of tritium can be made avajlable for propulsion systems before commercial electric fusion reactors with excess tritium breeding capacity are operational.

If the same trend occurs during the development of fusion power as with fission power, propulsion systems will play an important role. Today. throughout the world, no less than 250 shipboard nuclear propulsion systems have been built. Although most of these are on military platforms. five are presently in service on civilian ships. Even before the recent rapid increase in oil prices, the trend was toward more nuclear ships, on the order of $300-500$ by the year 2000 . In addition, prospects for aircraft nuclear propulsion systems will probably be re-examined, and as earth-orbit shuttle services become operational, earth-lunar and interplanetary missions will be considered.

Tradeoffs between fossil-fueled and nuclearfueled propulsion will continue to be difficult to make because of the potential environmental hazards of nuclear reactors. As a power supply for terrestrial propulsion, nuclear fusion has the advantage over nuclear fission of releasing far less radioactivity to the environment in case of an accident. The radiological hazard of the fusion reactor power systems considered here is primarily due to the tritium fuel supply and the structure-associated radionuclide inventory in the blanket. A $0.1-\mathrm{kg}$ tritium mass in the fuel supply amounts to $10^{6} \mathrm{Ci}$ of uniquely low biological hazard activity and represents 2 million horsepower hours ( 1.5 million $\mathrm{kWh}$ ) of energy. Moreover, the tritium may be stored in high-integrity containers. The induced activity in the reactor structure would be nonvolatile (i.e., would constitute only a very localized biological threat), and amount to only about $10^{7} \mathrm{Ci}$. This is to be contrasted with the $10^{10} \mathrm{Ci}$ of volatile fission products associated with a fission power source of comparable rating $\left(10^{9} \mathrm{~W}\right.$ thermal, or $3 \times 10^{5}$ mechanical horsepower).

A fusion power system for propulsion must be designed for minimum specific volume and mass. Thus, the reactor must operate with a high first wall loading. A laser fusion propulsion system will be superior to a magnetic fusion propulsion system because its specific volume and mass can be more readily reduced by increasing the pulse repetition rate and the first wall louding. A summary of the performance characteristics of advanced propulsion sycaems for several applications is presented in Table 1-4.

Use of laser fusion for marine propulsion offers the lowest technical risk. The listed marine requirements in Table $1-4$ appear to be well within projected efficiencies and scaling laws for laser fusion power systems. The more spacious and mass tolerant shipboard environments permit use of a helium cooled, graphite moderated version of a laser fusion reactor operating on a closed Brayton cycle. This reactor system will be radioactively cleaner than a liquid-metal-cooled system. Moreover, the use of helium will allow high temperatures and avoid the problems associated with liquid metals in water environments. From gravitational considerations, the first wall of the reactor should be a dry wall rather than a wet or fluid wall concept. Lithium will not be required because this high-performance reactor will operate on a parasitic deuterium-tritium fual cycle.

As with marine propulsion, some experience has been gained in the development of advanced nuclear reactors for aircraft propulsion. This experience is embodied in what remains from the Aircraft Nuclear Propulsion (ANP) project which was terminated in the early 1960's. This program was plagued with unpredictable technisal problems associated with the helium and liquid-metal-cooled reactors of that era and various components such as air-heat exchangers, bearings, pumps, seals and the reactor-shield assembly. This, along with uncertain cost benefits and the potential of public unacceptability of the risk, led to the Jemise of the program. The latter problem will be shared by airborne fusion systems to some degree.

Aircraft propulsion systems powered by laser lusion reactors do not appear to be an attractive alternative to chemical fueled or fission powered

Table 1-4. Summary of propulsion system characteristics.

\begin{tabular}{|c|c|c|c|c|}
\hline Application & $\begin{array}{c}\text { Technical } \\
\text { risk }\end{array}$ & Power $^{a}$ & $\begin{array}{c}\text { Specific } \\
\text { mass, } \\
\mathrm{kg} / \mathrm{kW},\end{array}$ & $\begin{array}{l}\text { Specific } \\
\text { volume, } \\
\text { m }^{3} / \mathrm{MW}\end{array}$ \\
\hline \multicolumn{5}{|l|}{ Marine } \\
\hline Navy & Moderate & $90 \mathrm{MW}_{\mathrm{s}}$ & 30 & BO \\
\hline Commercial & Low & $90 \mathrm{MW}_{\mathrm{s}}$ & 90 & 200 \\
\hline Airbome & High & $360 \mathrm{NW}$ & 0.4 & 0.6 \\
\hline Space & Highest & $130 \mathrm{GW}$ & 0.05 & $\begin{array}{l}\text { Not } \\
\text { critical }\end{array}$ \\
\hline
\end{tabular}


systems. The fusion plants will require a much larger specific volume and mass than current chemically fucled propulsion plants. The portions of the fusion power plant whose weight and volume must be traded off against the chemical fuel load include the laser system, pulsed power supply, reaczor, coolant and payload shielding. Preliminary calculations show that it will be difficult for the reactor alone to meet the mass and volume constraints, even for first wall thermal fluxes as high as $10 \mathrm{MW} / \mathrm{m}^{2}$.

Two space applications of fusion propulsion are being considered: an earth-moon shuttle or ferry and an interplanetary space craft for manned missions. These applications can take advantage of the enormous specific impulse* relative to that of chemical rockets. Nucieons are accelerated in fusion reactions to velocities on the order of $10^{7} \mathrm{~m} / \mathrm{s}$, making a specific intupulse on the order of $10^{6} \mathrm{~s}$ obtainable in principle. Chemical rockets have specific impulses limited to less than $450 \mathrm{~s}$. Fission systems will be limited to specific impulses of less than $2000 \mathrm{~s}$.

The ability of the space craft to move through space is normally measured in terms of $\Delta V$. This index is the sum of all propulsive energy required for translations between stable orbits throughout the entire mission in units of feet per second. $\Delta V$ is a function of the specific impulse of the propulsion system and the payload and spacecraft structure fractions. Approximate $\Delta V$ 's for various missions are given in Table !-5.

Chemical systems have $\Delta V$ 's most suitable to near-Earth operations because of their low specific impulse. For example, if a chemical system were designed to make a manned Venus reconnaissance, the payload fraction would be negligible. Even with an advanced fission/chemical staged rocket (nuclear stage specific impulse $\sim 1200 \mathrm{~s}$ ) the payload would only be a few percent of the total vehicle weight.

-Specific impulse = impulse per unit wejght; i.c., (ma) $\Delta \mathrm{t} /(\mathrm{mg})$ $=(\Delta v / \Delta t) \Delta t / g=\Delta v / g$ (unit is ime).

Table 1-5. Spacecraft capability required for various missions.

$$
\text { Mision } \Delta \mathrm{V}, \mathrm{fps}
$$

Eccape from Earth

$40 \times 10^{3}$

Ronnd trip Earth orbit to moon aurface

$40 \times 10^{3}$

Yenus manned reconnaissance

$150 \times 10^{3}$
Reasonable translunar shuttle times should be on the order of 24 hours. Fission systems with $J V$ 's on the order of $43,000 \mathrm{fps}$, i.e., 3,000 ips more than the minimum required, could make the trip in 24 hours. Fusion systems with abundant $\Delta V$ could make the trip so fast that local maneuvers would consume most of the transit time. A high-performance vehicle, powered by a laser fusion propulsion system has been postulated. Figure 1-10 gives the calculated performance for a system that produces $130 \mathrm{GW}_{\mathrm{t}}$. For a super-high-performance propulsion system of this kind, interplanetary trajectories may be quite accurately calculated by neglecting solar and planetary gravitation effects. As shown in the figure, a round trip from Earth to any point in the solar system may be made in less than one year by a system that carries a 100-ton payload and has a propulsion system mass of 300 tons. An initial fuel loading of 200 tons of deutcrium is sufficient for such maximum missions. Interstellar missions of a few decades transit time are also feasible.

The initiation of a sustained series of fusion microexplosions within a thrust chamber consisting of an asymmetric magnetic mirror is the essence of laser fusion rocket propulsion. The magnet geometry shown in Fig. $1-11$ is simllar to that used in mirror plasma confinement except that charge particle confinement is neither required nor desired. The thrust of the vehicle is produced by the redirection of plasma debris from the microexplosions

Closest approach distances to Earth

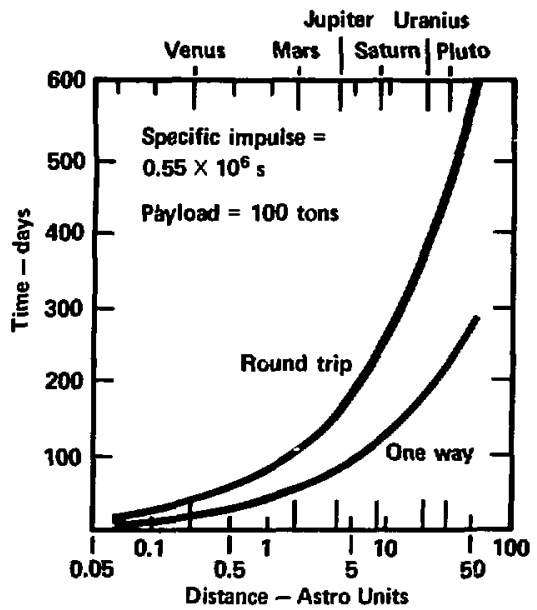

Fig. 1-10. Extraternestrial laser-fuslon-powerid vehicle performance. 


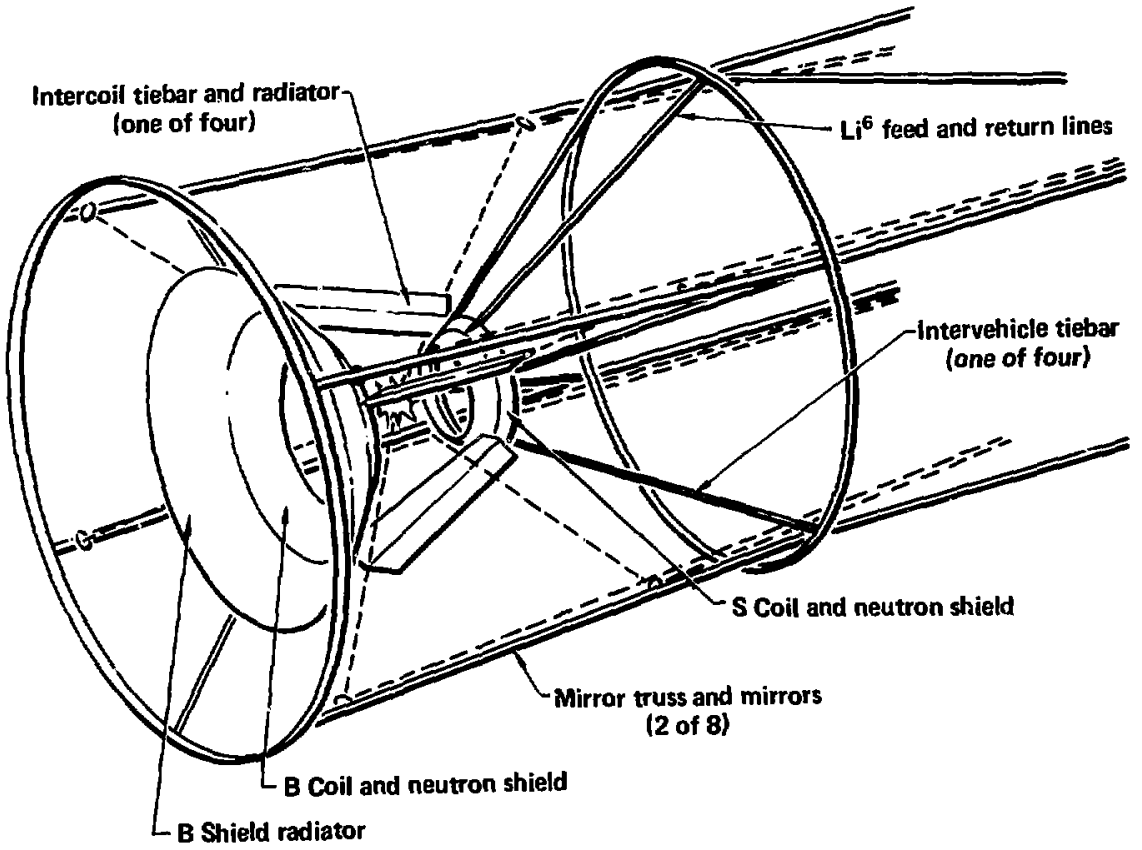

Fig. 1-11. Thrust chamber.

through the larger of the mirror loss cones, and out the rear of the spacecraft.

Neutrons and $x$ rays produced in the fireball are worse than useless; if intercepted, their energy must be rejected to outer space. The target design must maximize the ratio of debris energy to neutron and $x$-ray energy while minimizing required laser energy. Vehicle mass is dominated by the need for heat rejection to space which must be done radiatively. Waste heat is developed in two ways: component inefficiency, and neutron and $x$-ray interception. The above factors dictate an open thrust chamber so that less than $2 \%$ of the solid angle seen by the microexplosion is occupied. Thus, while charged particles are redirected by the magnetic mirror providing thrust, most of the unwantad neutrons and $x$ rays escape to space harmlessly. Only $2 \%$ of the neutrons and $x$ rays hit the radiation shields for the magnetic-field-producing superconducting coils, the payload shadow shield or the laser beam mirrors. However, even though this $2 \%$ represents a large heat rejection load, it has some value because the neutrons breed some tritium onboard in the lithium shields.

At present, the optimal design for a spacecraft laser fusion target (intermediate $\rho \mathbf{R}$ design) consists of deuterium with just enough tritium to facilitate ignition. Such a target burns far more efficiently than low $p R$ pellets; thus a smaller specific laser size will be needed. The laser reexcitation energy could be produced by directly converting the energy from the interaction of the charged plasma debris with an induction coil. Between 50 and $70 \%$ of the fusion energy is in charged debris from the microexplosion.

Because marine propulsion appears to be the easiest system to adapt to laser fusion and the one that has the best chance of early commercial success, we believe that studies of marine systems should proceed in tandem with laser fusion technical development. These studies should include the impact of a laser fusion reactor on hull design as well as the impact of potential spinoffs on the development of small stationary power plants. 


\section{References}

1. Laser Program Annual Report-1974, Lawrence Livermore Laboratory, Rept. UCRL-50021-74 (1975).

2. J. A. Maniscalco, "Fusion-Fission Hybrid Con- cepts for Laser Induced Fusion," Nucl. Technol. 28, 98 (1976).

3. A. G. Cook and J, A. Maniscalco, "Uranium233 Breeding and Neutron Multiplying Blankets for Fusion Reactors," Nucl. Technol. 30, 5 (1976). 


\section{CENTRAL STATION ELECTRIC POWER WITH LASER FUSION}

\section{A Comparison of Long-Range Sources of Electrical Energy}

The criteria used to compare laser fusion to other long-range sources of electrical energy include abundance, social costs, technical feasibility, and the cost of electricity. The term "social costs" is used to denote burdens borne by society that are not directly reflected in the cost of electricity. These include health risks. environmental issues, safety. and nuclear weapons proliferation. The technical feasibility of a future source of electrical energy can be assessed by the degree of engineering complexity and the amount of advanced technology required for high-average-power operation in an efficient manner. All of the long-range sources of energy are cost intensive. As a result, the cost at which they produce eiectricity is dominated by their capital costs and plant capacity fartors.

\section{Social Costs}

The generation of electricity inevitably results in risks to human health and adverse effects on the environment. A meaningful assessment of producing electricity with laser fusion requires that its social costs be compured to those of alternative sources of e:tergy. The health effects considered in the comparison involve prompt and delayed deaths, genetic diseases, serious illness, and discomfort. Environmental effects range from land use and resource depletion to world-wide climate changes. Some of the social costs, such as improving safety and reducing air pollution, are reflected in the cost of electricity; however, in general the social costs show up as hazards to life and damage to the environment.

Characterirtics of inertially confined fusion power plants on a deuterium-tritium fuel cycle that signlicantly affect their social costs include:

- High-energy neutrons which cause structural damage and activation

- Kilogram quantities of tritium present in the power plant

- Large lithium inventories

- Electricity generation via thermal energy conversion

- A self-contained fuel cycle

- Large reactor struclures

In Table 2-1 we have quantified the most important social costs that result from a laser fusion power plant with the characteristics presented above. Here, we compare laser fusion with the long- range alternatives (breeders, solar energy, and magnetic fusion) and the major existing producers of electricity (coal and LWRs). The majority of the entries in Table 2-1 were obtained from an ERDA sponsored study recently completed at Battelle Northwest Laboratories. 1 The majo: differences between laser and magnetic fusion result from our contention that the problems ussociated with fusion neutron damage and activation can be reduced by an order of magnitude in a laser fusion system. A reactor concept that emphasizes this feature is presented later in this chapter.

\section{Land Use}

Solar and coal-fueled power plants use an order of magnitude more land than the other energy producers. The large variance in land use for solar power plants results from the differences in the degree of insolation and whether thermal-electric or photovoltaic systems are used. The fuel needs of a $G W_{\mathrm{e}}$ coal plant require the mining of some 100 to 400 acres of land each year. At presc: uranium ore concentrations $(0.2 \%)$, some 20 to $5^{r}$ : :res of land must be mined annually for a $G W_{\mathrm{e}} \mathrm{LWR}$. As highgrade uranium ore is depleted, the land requirements for an LWR wil] approach and may even surpass those of coal.

\section{Transportation Requirements}

$A \mathrm{GW}_{\mathrm{c}}$ coal-fired power plant requires more than 10,000 tonnes of coal per day of operation. As a result coal transportation presently accounts for approximately $10 \%$ of the freight car haulage in the United States. The large difference in the transportation reyuirements for laser and magnetic fusion primarily results from the requirement to replace the neutron-damaged blanket structure in the mangetic fusion power plant periodically (I to 5 years).

\section{Gaseous Erfuents}

This category identifies air pollution problems that contribute to the overall health risk. Air pollution has long been known to be a cause of illness and death as well as discomfort. The prinicipal health effects of coul-fired plants are thought to result from sulfur-related pol]ulants. These account for approximately $75 \%$ of the gaseous effluents of a. coal plant with scrubbers. 
Table 2-1. The social costs (health, environment and safery) of generating $1 \mathbf{G W}_{e}$ of electricity.

\begin{tabular}{|c|c|c|c|c|c|c|}
\hline & Cod & LWR & $\begin{array}{c}\text { Breeder } \\
\text { reactor }\end{array}$ & Solar & $\begin{array}{c}\text { Magnetic } \\
\text { fusion }\end{array}$ & $\begin{array}{l}\text { Laser } \\
\text { fusion }\end{array}$ \\
\hline \multicolumn{7}{|l|}{ Land use (acres) } \\
\hline $\begin{array}{l}\text { Fower plant } \\
\text { Fuel cycle (30 yeas life) }\end{array}$ & $\begin{array}{l}300 \\
3,000-12,000\end{array}$ & $\begin{array}{l}100-200 \\
600-12,000\end{array}$ & $\begin{array}{l}100-200 \\
15\end{array}$ & $\begin{array}{l}6,000-19,000 \\
0\end{array}$ & $\begin{array}{l}100-200 \\
0\end{array}$ & $\begin{array}{l}100-200 \\
0\end{array}$ \\
\hline $\begin{array}{l}\text { Transportation requirements } \\
\text { (tonne/yr) }\end{array}$ & $3 \times 10^{6}$ & 200 & 23 & Unknown & $100-400$ & 10 \\
\hline \multicolumn{7}{|l|}{ Gascous effuents } \\
\hline $\begin{array}{l}\text { Nonradioactive (tonne/yr) } \\
\text { Rediouctive (Ci/yr) }\end{array}$ & 40,000 & 21 & 5 & Unknown & $\mathbf{0}$ & 0 \\
\hline $\begin{array}{l}\text { Tritium } \\
\text { Other }\end{array}$ & $\begin{array}{l}0 \\
0\end{array}$ & $\begin{array}{l}15,700 \\
350,000\end{array}$ & $\begin{array}{l}2000 \\
150,000\end{array}$ & $\begin{array}{l}\mathbf{0} \\
\mathbf{0}\end{array}$ & $\begin{array}{l}1060 \\
0\end{array}$ & $\begin{array}{l}1000 \\
0\end{array}$ \\
\hline \multicolumn{7}{|l|}{ Solid waste } \\
\hline $\begin{array}{l}\text { Nonnendionctive (tonne/yr) } \\
\text { Rudionctive (m } 3 / y r)\end{array}$ & $1 \times 10^{6}$ & $\begin{array}{l}91,000 \\
340-1100\end{array}$ & $\begin{array}{l}9,100 \\
450-1200\end{array}$ & $\begin{array}{l}\text { Unknown } \\
0\end{array}$ & $\begin{array}{l}\text { Negligible } \\
\mathbf{4 5 0}\end{array}$ & $\begin{array}{l}\text { Negligible } \\
30\end{array}$ \\
\hline \multicolumn{7}{|l|}{ Sufety } \\
\hline $\begin{array}{l}\text { Accident consequences } \\
\text { Accident probabilliny }\end{array}$ & $\begin{array}{l}\text { Low } \\
\text { High }\end{array}$ & $\begin{array}{l}\text { Very high } \\
\text { Very low }\end{array}$ & $\begin{array}{l}\text { Vory high } \\
\text { Very low }\end{array}$ & $\begin{array}{l}\text { Very low } \\
\text { Very low }\end{array}$ & $\begin{array}{l}\text { Low } \\
\text { Very low }\end{array}$ & $\begin{array}{l}\text { Low } \\
\text { Very low }\end{array}$ \\
\hline Proliferation hazord & No & Yes & Yes & No & No & No \\
\hline
\end{tabular}

The release of $\mathrm{CO}_{2}$ from the coal burning process may pose an even more serious long-term problem by causing significant climate changes. $\mathrm{CO}_{2}$ in the atmosphere heats the earth by the so-called greenhouse effect because it is transparent to the incident solar radiation bu :ibsorbs the heat reradiated from the earth. W. witer the effect of increasing the amount of $\mathrm{CO}_{2}$ in the atmosphere will combine with natural changes and other causes to produce an adverse effect on our climate remains to be demonstrated. This uncertainty could take on an overriding significance in the comparison between coal and nuclear fission. Specifically, it argues against complete reliance on coal at this time.

Solar power plants will not release any significant quantities of gaseous effluer.ts; however, they can be expected to adversely affect the local climate by changing the absorption and reradiation properties of the earth's surface in their vicinity. Solar-energy collectors that would cover half the surface in any given area could be expected to alter the temperature and the surface wind conditions.

Fusion reactors will contain kilogram quantities of tritium within their confines, and it is inevitable that some of this radioactive material will be released to the environment in gaseous form. Battelle ${ }^{2}$ has estimated that the tritium release from a $\mathbf{G W}_{\mathrm{e}}$ Tokamak fusion reactor will be approximately $1000 \mathrm{Ci} / \mathrm{yr}$. We have assumed the same tritium release for laser fusion. The potential health risk of this relatively small amount of tritium can be put into perspective by noting that the natural production of tritium by cosmic rays yields a steady-state inventory of $10^{\mathrm{B}} \mathrm{Ci}$, which accounts for $0.02 \%$ of the radiation dose we receive from natural sources. Tritium is a radioactive material with a uniquely low biological hazard. It has a halflife of 12.26 years and emits a weak beta $(18.6 \mathrm{keV})$. Tritium is readily absorbed by biological systems, but it has a very short biological half-life: less than 1 day for $\mathrm{T}_{2}$ and about 8 days for $\mathrm{T}_{2} \mathrm{O}$.

The relatively large releases of tritium from fission reactors occur when the spent reactor fuel is reporcessed. However, these releases of tritium are small compared to the gaseous fission products (primarily ${ }^{85} \mathrm{Kr}$ ) that are also released from the irradiated fuel.

\section{Solid Radioactive Waste}

An LWR uses 30 tonnes of fuel per year. At reactor shutdown, a tonne of spent fuel contains $3.0 \times$ $10^{8} \mathrm{Ci}$ of radioactive wastes. After 10 years, its radioactivity has decayed to $3 \times 10^{5} \mathrm{Ci}$. The highlevel wastes (primarily fission products) constitute the majority of the radioactivity for the first few hundred years. However, high-level wastes constitute less than $1 \%$ of the volume of radioactive material listed in Table 2-1. The major health hazards from the remaining low-level wastes result from the actinides. These transuranic elements, in 
general, exhibit very long half-lives and are extremely toxic.

The major source of solid radioastive waste in fusion power plants is neutron activation of the blanket structure. When exposed to first-wall fusion neutron fluxes that support acceptable power densities ( $>1 \mathrm{MW} / \mathrm{m}^{2}$ ), these blanket structures are expected to survive from 1 to 5 years. ${ }^{3}$ Later in this chapter, we present a laser fusion reactor concept that reduces the fusion neutron flux on the blanket structure by an order-of-magnitude, thereby allowing us to consider structures that could last the lifetime of the power plant.

\section{Safety}

Mining and transportation accidents runsti ie the major health hazards from coal-fired power plants. These events have been estimated to cause

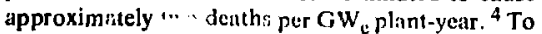
date the sufety Itcord of LWRs has been excellent. No accidents causing death have occurred in more than 200 reactor years of commercial operation. However, the fact that fission reactor accidents could have serious consequences for public health has long been recognized. The Rasmussen Reactor C. icty Study ${ }^{5}$ estimated that an extremely serious accident (a sequence of events leading to core meltdown and breaching the secondary containment) under very adverse conditions would kill as many as 3,000 to 4,000 people in a few weeks and tens of thousands of people over a 30-year period. The probability of this type of accident occurring is so low that the report estimates the accidental death rate from LWR operation to be more than two orders of magnitude lower than for coal. Nevertheless, there are some people who believe that the consequences of a fission reactor accident are so great that the risks are unacceptable no matter how low the probability of occurrence.

A comprehensive accident analysis of fusion power plants cannot be made yet because detailed plant designs and site descriptions are required. However, preliminary assessments of possible accidents indicate that both the probability and consequences of a catastrophic accident in a fusion reactor would be substantially lower than for a fission reactor. Features of fusion systems that lead to this conclusion are:

- Significantly less radioactive byproducts; i.e., no fission products or actinides

- The relatively low biological hazard of tritium (the release of kilogram quantities of tritium would result in maximum doses in the 10's of rems-well below lethal levels)

- No afterheat cooling problem in case of a loss-of-coolant accident
- No nuck ar runaway or power excursion from a reactivity insertion or criticality accident.

Liquid metal fires appear to be the most probable cause of a serious accident in a laser fusion power plant. Combustion of the liquid metal in the blanket could release kilogram quantities of tritium ano much larger quantities of chemically hazardous hydroxides. The radioactivity bound up in the blanket structure would not be vaporized because the flame temperature of liquid lithium is lower than the vaporization temperature of sandidate structural materials. The toxic $\mathrm{LiOH}$ produced in the combustion of lithium could be more lethal than the tritium; LiOH is present in the smoke, and it causes lung burns when inhaled. Therefore, its lethal consequences can be avoided with a gas mask and protective clothing, and it can be expected to dissipate as rapidly as the smoke from a conventional fire

\section{Proliferation}

This entry in Table 2-1 emphusizes the fact that fusion does not require fissile materials which can be used to make nuclear weapons. Thus, the following problems associated with fission reactors can be avoided:

- Increasing nuclear weapens proliferation

- Diversion of nuclear weapons material

- Sabotage of nuclear facilities

Proponents of fission reactors argue that the hazard of nuclear weapon proliferation exists independent of American nuclear power. They point out that restrictions on U.S. nuclear programs or expor1s simply reduce our influence over nuclear power developments abroad, thus diminishing our ability to deal with the important problerss of sabotage and diversion by terrorist groups.

In summary, a cursory comparison of the systems and data in Table 2-1 seems to indicate that the social costs of solar energy, magnetic fusion, and laser fusion are significantly lower than alternative sources. A more precise comparison and rankin? of these three sources is not possible at this time for two reasons. First, the technical design of largescale solar or fusion power plants has not progressed far enough for a detailed analysis of social costs. Second, the social costs themselves vary with time and present a difficult and complex pattern of interrelated factors.

The central point to be recognized at this initial stage is that social costs, which are intimately linked to the cultural and political processes in society, may become the controlling factors in the development of energy technologies. The adoption of a relatively expensive system (within a factor of two) in terms of capital investment and electricity cost to 
the consumer may. in terms of social costs. be the wisest course of action for society in meeting future energy needs.

\section{Technical Feasibility of I.aser Fusion}

Fusion, like all advanced sources of electrical energy, is expected to be technically more complex than existing LWR or fossil-fueled power plants. The technical feasibility of laser fusion power plants rests primarily with the developments required for high-average-power operation in an efficient and reliable manner. $A s$ an alternative to magnetic confinement. laser fusion offers features that could significantly reduee its technical complexity, thereby reducing the amount of advaneed technological development that will be required to produce a tcchnically successful fusion power plant. These fentures include:

- No magnets* (asterisked items are design. dependent)

- Sizing Dexibility (i.e., lower power units)

- Possibility of multiple chambers to provide higher availability

- Flexible geometry

- No dircel conversion required

- Reduced vacuum pumping rcquirements*

- Reduced radiouctivity from neutron activation*

- Reduced neutron damage to structural materials*

Later in this chapter, we deseribe a reactor design concept that features all of the advantages listed abovc. Before these advantages can be realized, the major technical problems associated with a laser fusion power plant must be solved. These include the development of:

I. A high-average-power driver with the required efficiency $(>I \%)$ and reliability $(>70 \%)$

2. A first wall able to withstand the effects of $x$ rays, debris, and neutrons from the microexplosion

3. Structural materials that can withstand the cumulative damage effects of high-energy neutruns and cyclical stresses

4. Final-focusing elements that can be placed far enough away from the mieroexplosion or protected so that they can last a reasonable length of time

5. The technology to cheaply mass-produce fusion targets

In the next section, we analyze and compare laser systems that have the potential to be high-averagepower drivers for a power plant. The material damage and final focusing element problems (2 through 4) will be dealt with in the final section of this chapter where we present the results of studies that have focused on identifying and analyzing reactor concepts for laser fusion.

In our development scenario for inertial confinement fusion (Appendix), we show that the. technology to cheaply mass-produce fusion targets could be some the pacing element in the engineering and commercial phases of development.

Solutions to the technical problems listed above will allow laser fusion power plants to efficiently produce electricity with high plant capacity factors. The overall efficiency of a laser fusion power plant can be evaluated through a basic power flow diagram like the one shown in Fig. 2-I. The simple expression shown in this figure gives the plant recirculating power fraction $\left(P_{i n} / P_{g}\right)$ as a function of laser efficiency $\left(\eta_{1}\right)$, pellet gain $(Q)$, thermal efficiency $\left(\eta_{1}\right)$. neutron energy fraction $\left(f_{n}\right)$, and blanket energy multiplication $\left(M_{H}\right)$; i.e.,

$$
\frac{P_{\text {in }}}{P_{E}}=\frac{1}{\eta_{L} Q \eta_{\mathrm{T}}(0.8 \mathrm{M}+0.2)}
$$

Recirculating power fraction is a basic figure-ofmerit for any power plant. For reference purposes. fossil-fueled power plants operate with recirculating powers less than $5 \%$ while the majority of present nuclear plants operate at or slightly above this figure. Laser system efficiency $\left(\eta_{L}\right)$ is a lumped parameter that includes power conditioning. medium regeneration, lasing, and optical transport efficiencies. It is defined as the ratio of focusable beam energy to electrical energy input. Blanket energy multiplication $\left(\mathrm{M}_{B}\right)$ is defined as the ratio of blanket thermal energy to fusion neutron energy. The overall system efficiency $\left(\eta_{\mathrm{s}}\right)$ of a laser fusion power plant can be related to the recirculating power fraction (RPF) by the equation:

$$
\eta_{\mathrm{s}}=\eta_{\mathrm{t}}(1-\mathrm{RPF})
$$

From the expressions for recirculating power fraction and system efficiency, it logically follows that the product of laser efficiency $\left(\eta_{\mathrm{L}}\right)$ and pellet gain (Q) should provide an excellent figure-of-merit for gauging the prospects of efficiently generating electrical power with laser fusion. This product, which we shall label fusion energy gain, represents the ratio of thermonuclear energy output to electrical energy input to the laser. Fusion energy gain is an extremely revealing performance parameter becausc economical power production with laser fusion will fundamentally lie with the pellet energy gains achieved and the overall efficiencies at which 


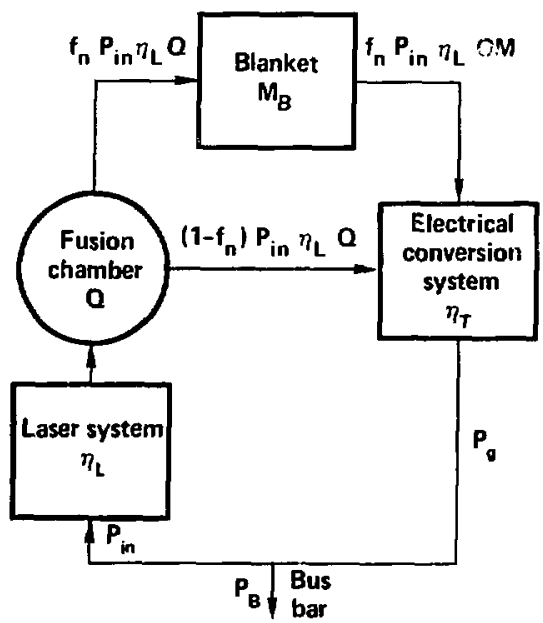

where:

$$
\begin{aligned}
& \eta_{\mathbf{L}}=\text { laser sysiem efficiency } \\
& \mathbf{Q}=\text { pellet gain }=\frac{\text { thermonuclear energy }}{\text { laser light energy }} \\
& \mathbf{f}_{\mathbf{n}}=\frac{\text { neutron energy }}{\text { thermonuclear energy }} \\
& \mathbf{M}_{\mathbf{B}}=\text { blanket energy multiplication } \\
& \eta_{\tau}=\text { electrical conversion efficiency } \\
& \mathbf{P}_{\text {in }}=\text { electrical power input to laser } \\
& \mathbf{P}_{\mathbf{g}}=\text { gross electrical power }
\end{aligned}
$$

$$
R P F=P_{i n} / P_{g}=1 / \eta_{L} O_{\eta_{t}}\left(1+f_{n}\left(M_{B}-1\right)\right)
$$

Fig. 2-1. I.aser fusion power how digpram.

large pulsed ignition sources can operate. In Fig. 22. the recirculating power fraction is plotted as a function of fusion energy gain for power plants with a blanket energy multiplication of 1.0 and several thermal efficiencies. Reference designs for laser fusion power plants have used recireulating power fractions in the neighborhood of $25 \%$. However, it may be difficult to cumpete economically at these high values because, in. general, plant capital costs scale with the gross electrical power while revenues scale with the net electrical power.

\section{Cost of Electricit!}

The cost of electricity from a power plant is del trmined by the revenue required to provide for a return on capital investment. the cost of fuel. and the cost of operation and maintenance. Like fission reactors, all potential long-range sources of energy will be capital intensive: therefore, the cost at which they produce electricity will be primarily deternined by their capital tharpes. The capital investment component of the cost of electricity is determined from the simple relationship:

$$
C_{\mathrm{I}}\left(\mathrm{mills} / \mathrm{kW} \mathrm{h}_{\mathrm{H}}\right)=\frac{(0.114) \cdot \mathrm{C}_{\mathrm{p}} \cdot \mathrm{R}}{\mathrm{C}_{\mathrm{f}}} \text {, }
$$

where

$C_{p}$ is the total capital cost (direct and indirect) of the plant in $\$ / \mathrm{kW}$

$\mathbf{R}$ is the fractional rate of retu: $\mathbf{n}$ on capital per year

$C_{f}$ is the capacity factor, defined as the ratio of electricity actually produced in a fixed period to the amount that would be produced if the plant operated continuously at full capacity

For a commercial power plant, the rate of return on capital must be sufficient to pay interest, allow for depreciation, provide a return on the equity, and pily income tax. This translates into an effective capital charge rate that varies depending on the linancial structure of the utility: $15 \%$ is fairly typical.

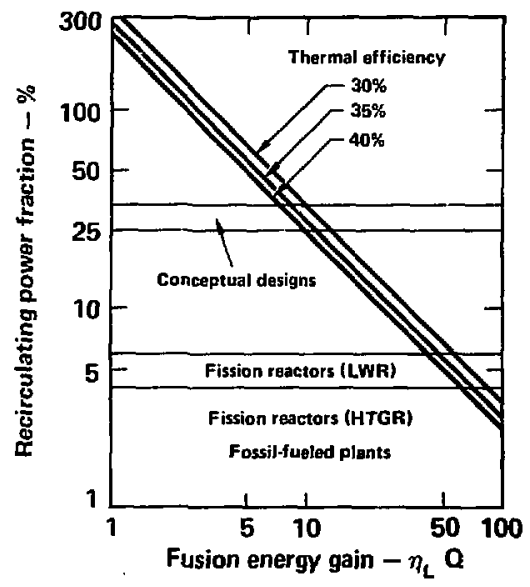

Fig. 2-2. Plant recirculating power requirements as a function of fusion energy gain. 
In Table 2-2, we present a recent Bechtel Corporation estimate 6 of the capital and operating costs (in 1976 dollars) of an I.WR ordered in 1976 for operation in 1985. The capital portion of the operating cost is based on a capital charge rate of $15 \%$ per yuar and a capacity factor of $70 \%$. The fucl costs are based on charges of $\$ 40 /$ lb for $\mathrm{U}_{3} \mathrm{O}_{4}(3.2$ mills $/ \mathrm{kWh}$ ), $\$ 100 / \mathrm{kg} / \mathrm{SW} U^{*}$ for uranium enrichment $(2.4 \mathrm{mills} / \mathrm{kWh})$, and $\$ 100 / \mathrm{kg}$ for fuel fabrication $(0.50 \mathrm{mills} / \mathrm{kWh})$. It is interesting to compare the cost of generating electricity from this capital intensive I.WR to that of its current competitor-is luel inlensive coul-fied plant. The cost of electricity in mills/kWh for a coul plant (with scrubbers) operating in 1985 breaks down as follows?:

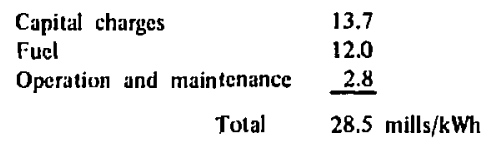

\section{Cost of Electricity from laser Fusion}

We have not performed a detailed cost analysis of a laser fusion power plant. Our cost analyses have

$\overline{\text { SWII }}=$ separative work unit.

Table 2-2. Capital and operaling costs of a $1200-M W_{e}$ LWR ordered in 1976 for operation in 1985 (1976 dollars).

\begin{tabular}{|c|c|}
\hline - Capital costs & $\operatorname{Cost}, 10^{6} s$ \\
\hline Nuclear steam supply system (NSSS) & 78 \\
\hline Other meclianiced & 101 \\
\hline Civil and structure & 142 \\
\hline Piping & 77 \\
\hline Instrumentation & 9 \\
\hline Electrical & 43 \\
\hline Total direct & 450 \\
\hline Indirect costs & $503(9$ yr) \\
\hline Total capitel cost & 953 \\
\hline - Capital cost per kW installed & $\$ 794 / \mathrm{kW}_{\mathrm{e}}$ \\
\hline - Total cost & Cost, mills/kWh \\
\hline Capitel & 19.42 \\
\hline Fuel & 6.3 \\
\hline Opernting and maintenance & 1.5 \\
\hline Total cost & 27.22 \\
\hline
\end{tabular}

primarily focused on identifying the economic constraints that will ultimately prevail. In line with this objective we have concentrated on providing upper bound estimates on the allowed costs of the laser and reactor portions of an economically attractive fusion power plant.

In Fig. 2-3 the allowed capital cost difference in $\$ / \mathbf{k W}$ between laser fusion and the reference LWR in Table 2-2 has been plotted as a function of the cost of uranium in $S / 1 b$. The following are the assumptions used to generate the curves in the rigure:

- The laser fusion fuel cycle accounts for 3 mills/kWh.

- Operation and maintenance costs for laser fusion are the same as those for the LWR (1.5 mills/kWh).

- Both power plants operate with a capacity factor of $70 \%$ and a capital charge rate of $15 \%$ per year.

For a laser fusion power plant with a system efficiency of $36 \%$, a fuel cycle cost of $3 \mathrm{mills} / \mathrm{k}$ Wh will allow a pellet fabrication cost of $0.03 \mathrm{e}$ per MJ of yicld. This allowed value translates to fabrication costs ranging from $12 \mathrm{e}$ to $\$ 1.20$ for pellet yiclds from 400 to $4000 \mathrm{MJ}$.

Some perspective for the relationships shown in Fig. 2-3 can be obtained by noting that uranium at $\$ 30 / \mathrm{lb}$ contributes $2.5 \mathrm{mills} / \mathrm{kWh}$ to the cost of electricity. This fueling cost is equivalent to a capital cost difference of $\$ 100 / \mathrm{kW}$ at a capital charge rate of $15 \%$ and a capacity factor of 0.7 . Estimates of reasonably assured supplies of high-grade uranium ore $(0.08$ to $0.2 \%$ uranium) indicate that there will be enough uranium at prices below $\$ 100 / 1 \mathrm{~b}$ to fuel LWRs through this century. At higher prices, lower

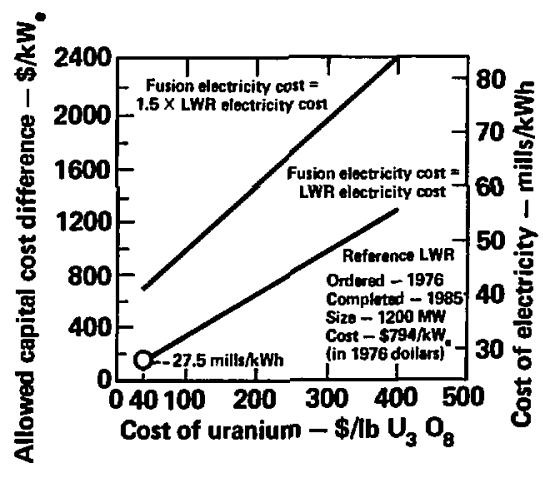

Fig. 2-3. Allowed copitel cost difference between an inertial confinement fusion resctor and an LWR. 
grades of ore could be used. However, their use would increase the mining and land use requirtments of LWRs to the point where they would surpass those of coal-fired plants. At uranium prices greater than $\$ 500 / \mathrm{lb}$, it may be possible to extract uranium from sea water. thus guaranteeing a virtually inexhaustible supply.

The nuclear steam supply system (reactor and primary coolant loop) of the reference LWR costs $5138 / \mathrm{kW}$ (indirect and direct costs) and it accounts for approximately $20 \%$ of the total cost. In Fig. 2-4 we have plotted the allowed cost of the nuclearisland equivalent of a laser fusion power plant (lasers and reactor system) as a function of the cost of uranium. These curves were generated by adding the capital cost differences in Fig. 2-3 to the cost of the LWR nuclear island. As such they provide an upper bound estimate of the allowed cost of the nuclear island equivalent of a laser fusion power plant. The results show that a $\mathrm{GW}_{\text {. laser fusion }}$ power plant can generate electricity at costs that are within $50 \%$ of present prices with laser and reactur systems whose combined costs are as much as $\mathbf{8 0 0}$ million dollars.

\section{Cost Comparisons With Breeders and Solar Electric Power Plants}

Capital costs for liquid metal fast breeder reactors (LMFBRs) are expected to be signilicantly higher than those of L.WRs. Direct estimates from the EPRI/ERDA cooperative design projects currently in progress indicate that LMFBRs will cost from $\$ 400$ to $\$ 600$ per $k W_{\mathrm{e}}$ more than an $L W R$, with fuel cycle costs ranging from 1.5 to 5 mills/kWh. ${ }^{8.9}$ By assuming a fuel cycle cost of 3 mills $/ k$ Wh we can use the information in Fig. 2-3 to determine the cost of electricity from L.MFBRs. The results show that breeders will generate electricity at costs that are 20 to $40 \%$ more than present prices and that they will not economically compete with LWRs until uranium prices are more than $\$ 120 / 1 b-w e l l$ into the next century. These economic results when coupled with the larger social costs associated with plutonium reprocessing and recycle argue strongly for derferring the commercial introduction of the LMFBR for 10 or 20 years, thereby allowing the $U$. S. to pursue a broader R \& D program. Such a program could provide a range of attractive choices with introduction dates extending into the next century.

Two processes for converting solar energy into electricity have been proposed: solar therma!electric and solar voltaic. Solar thermal-electric systems use fixed or movable arrays of mirrors to reflect the sun's energy onto a central tower, which heats a working fluid that produces electricity in a conventional steam cycle. Present estinates indicate that these power plants will cost about $\$ 2000 / \mathrm{kW} .2$ to 3 times nore than an I.WR. ${ }^{10}$ Moreover, solar power plants will exhibit a capacity fuctor of less than $30 \%$ because of the diurnal variation of insolatien. As a result solar thermal-electric power plants can be expected to produce electricity that costs some six to nine times more than present prices.

Solar voltaic systems use crystals ( silicon. gallium arsenide. and polycrystalline substances) to directly convert solar energy into electricity. Conversion efficiencies of aboui 10\%, have been achieved. Nevertheless photovoltaic methods are presently far less competitive than solar thermal-electric systems: however. substantial improvements may be possible with new teshniques that could substantially reduce the cost of collectors and increase their efficiency. The cost of solar collectors must be reduced to about $\$ 1 / \mathrm{ft}^{2}$-several hundred to a thousand times less than the present cost before they can produce competitively priced electricity.

From the cost comparisons presented here, it is obvious that the electricity generated by any of the long-range sources of energy will be significantly more expensive than electricity is now. However, within a factor of two, the cost of energy in the U.S. economy is much less critical than assured supply. Present total energy costs are only $5 \%$ of the U.S. gross national product, and it has been estimated that a doubling of energy costs would result in a 2 or $3 \%$ reduction in consumption with little effect on economic growth in the long run. ${ }^{10}$

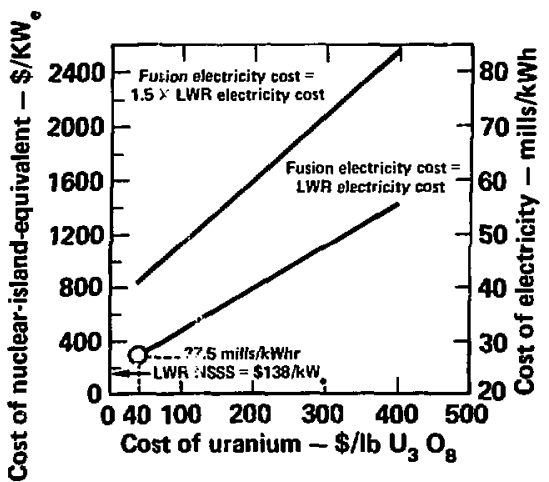

Fig. 2-4. Miximum allowed cost of nuclear-island-equirglent of inertial confinement fesion reactor. Assumptions:

- ICF muclear islanil includes driver, reactor vessel, and primary coolant loop

- ICF fuel cycle cost $=3$ mills $/ \mathrm{kWh}$

- Nuclear island cost depietes allowed cost difference 


\section{Lasers for Laser Fusion}

The purpose of the driver systens in an inertially confined fusion power plant is conceptually simple. It must taike a small fraction of the steady state electrical power produced by the reactor, form it into pulses of energy at the rate of 1 to 10 per second, concentrate the energy into pulses a billionth of a second in duration, and concentrate the energy in space by projecting it about $10 \mathrm{~m}$ onto a target pellet a millimeter or so in diameter. Three types of driver systems can potentially accomplish this concentration of energy in time and space: ion beams, clectron beams, and photon beams.

It is premalure to thoroughly compare these different systems; we shall not do so here. We will, however, indicate why we feel that lasers are a desirable choice for the near, intermediate, and fartern applications. Of course, it is also too early to discard any contender, for each system has different advantages and disadvantages that will determine the system's development. For example, suitable heavy ion accelerators of $\leqslant 100 \mathrm{GeV}$ beam energy appear to be technologically feasible. The ion beam system draws on the most mature physics and engineering base and is therefore easier to visualize in full scale. The efficiency is projected to be high, $>10 \%$, which will allow a low recirculating power fraction. Accelerators would have the capability for high repetition rate, reliability, and operating lifetime required for a commercial power plant. Unfortunately, all existing heavy ion accelerators are relatively low-current devices. Consequently, it is uncertain that the high beam current required for inertial confinement fusion can be accelerated, transported, and focused onto the target. Moreover, large accelerators are quite expensive, and significant research will be required to determine whether high-current accelerators can be built at reasonable cost. Nevertheless, the potential high efficiency, high-average-power capability and moderate technical risk compel us to examine this option carerully.

Electron beams are also attractive alternatives from the standpoint of size, simplicity, and efficiency. Progress is being nude at Sandia in scaling 20- to 30-ns E-beams io stibstantial energies per pulse. The crucial problem is the inability to focus beams of electrons from far nough away to allow the generating diode to survive. Either a breakthrough in the physics or an engineering innovation allowing renewable or regenerative electron guns is required. The expected efficiency is attractive and the average power capability is fine as long as the diode is not destroyed on every shot. The electron beam needs a focusing element that can concentrate the energy onio the pelfet from a safe distance.

$A$ laser system appears to be an ideal way to concentrate energy in space from a safe distance. The system might take the form of a gas laser driven by a broad area long pulse width electron beam: such $E$ beams are technologically less demanding.

Laser-induced fusion can be approached using many different target concepts which, in terms of projected applications, dictate a range of lasersystem performance requirements. During the next few years, the viability of the various target concepts will be evaluated through fusion experiments (using the Janus, Argus, and Shiva laser systems) and target design itcration. Our target calculations and reactor system studies have established preliminary laser system performance requirements for commercial electric power production. The nominal requirements are

$\begin{array}{ll}\text { - Wavelength } & 250-2000 \mathrm{~nm} \\ \text { - Pulse duration } & 1-10 \mathrm{~ns} \\ \text { - Pulse energy } & 300-3000 \mathrm{~kJ} \\ \text { - Pulse power } & >200 \mathrm{TW} \\ \text { - Pulsc repetition rate } & 1-10 \mathrm{~Hz} \\ \text { - Average power } & 1-10 \mathrm{MW} \\ \text { - Overall efficiency } & >1 \%\end{array}$

These requirements continue to evolve, reflecting new knowledge in target and laser physics, materials and laser engineering, systems analysis, and economics. Important constraints are encountered in each arca. For example, in the case of the laser wavelength, the physics constraints favor short wavelengths to compress the pellet with the least energy. Short wavelengths prompt investigation of transitions between electronic energy states. However, the material and technological constraints limit us to consideration of wavelengths larger than about $250 \mathrm{~nm}$ because of severe twophoton optical absorption effects. Operation with short wavelengths also impacts the system and economics constraints because the short wavelength gas lasers we know today are somewhat less efficient than infrared lasers. For each laser parameter, there is a similar balance and interplay between the physics technology and systems constraints, which often seems to leave very little room in parameter space to realize an acceptable solution. In fact, no current laser concept is perfect, but we have identifjed some promising candidates.

The medium in which the laser beam is amplified significantly affects the laser's ability to satisfy the fusion power plant requirements. Depending on the medium, stimulated emission can occur on 
- Electric dipole allowed transitions

- Electric dipole forbidden transitions such as magnetic dipole or electric quadrupole transitions

- Optically induced transitions such as Raman scattering or frequency tripling

Laser media of the first type, including the rare-gas and rare-gas-halogen excimers, exhibit stimulatedemission cross sections in the range $10^{-16}$ to $10^{-17}$ $\mathrm{cm}^{2}$, with upper-laser-level radiative lifetimes of tens of nanoseconds and instantaneots stored energy densities of less than $\mathbf{J}$ /liter under efficient excitation conditions. In contract, laser media of the second type, including iadine, Group Vl-A atoms, and rare-earth molecular vapors, exhibit stimulated-emission cross sections in the range $10^{-18}$ to $10^{-20} \mathrm{~cm}^{2}$, with upper-laser-level radiative lifetimes up to hundreds of microseconds, and instantaneous stored energy densities up to tens of J/liter under reasonable pumping conditions. The third type, including Antistokes electronic Raman Scattering in $\mathrm{Hg}_{2}^{*}$, is more difficult to evaluate since it is in an early research stage. The radically different physical and kinetic properties of the first two types of laser media dictate distinctly different lasersystem concepts and technologies.

Of the three types of advanced laser media sketched above, the photolytic Group VI-A laser has the largest information base. An evaluation of the efficiency and scalability of one version of this laser follows in a later section, but first it is valuable to shift focus from the physics considerations to the systems view point of evaluating a laser candidate on the basis of overali efficiency.

There are two important and separate aspects to a large laser system: efficiency and scalability. A power plant laser system of high efficiency will have lower recirculating power needs and lower capital equipment costs. For the photolytically pumped Group VI laser, the efficiency depends on losses due to power conditioning. generation of the electron beam, optical pump photon production, coupling to the lasing molecule, optical extraction, and the pumping, cooling, and chemical regeneration of the flowing gases.

Scalability refers to the ability to reach the high pulse energies required by a laser fusion reactor with power amplifier modules of reasonable size. A laser built with a large aperture and pulse energy lowers costs by reducing the number of beams and components. Thus, in a commercial system, 10 beams in a few beam clusters would be superior to 100 individual beams. The scalability depends on mirror and e-beam foil damage limitations, pump constraints, now and gas uniformity constraints, parasitic suppression, thermal distortion, and arrival at a configuration that simultaneously pushes all the important technology barriers.

In any given laser system. it is usually possible to trade off efficiency vs scalability to achieve an optimal system. It now appears that achieving high efficiency rather than high energy per pulse or high average power will be the most difficult for visible gas laser systems.

There are a number of considerations in the determination of overall laser system efficiency which are often overlooked. A high average power gas laser system can be thought of as three separate subsystems: optical conditioning, electrical conditioning, and gas conditioning (Fig. 2-5). The optical train transports the beam from the final amplifier aperture through isolators that prevent return of reflected light, through a port to the final focusing element, and then through the chamber background gas to the target pellet. Losses include absorption and scattering of lenses and mirrors, as well as beam-shaping and diffraction-spillage losses. A typical optical train might require ten optical elements. The optical conditioning efficiency is the ratio of power on target $P_{0}$ to the laser power $P_{L}$

$$
\eta_{\mathrm{oc}}=\mathrm{P}_{0} / \mathrm{P}_{\mathrm{L}}
$$

In a well designed system, the optical efficiency might be in the range of $90-95 \%$.

The electrical conditioning efficiency is the ratio of laser radiation produced to the electrica! power provided for the pulse forming network. It is the product of the component efficiencies for the pulse forming network, E-beam, optical pump, quantum

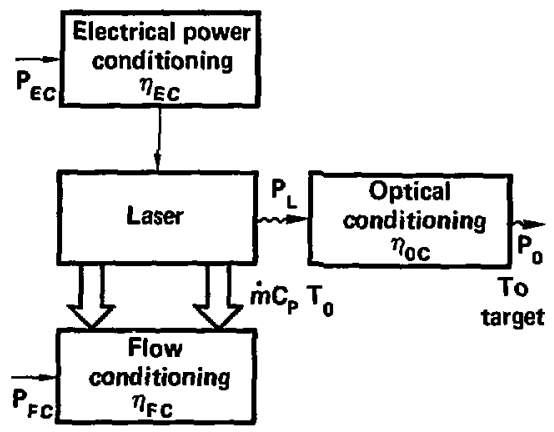

Fig. 2-5. Laser system power now diagram. 
transfer, and optical extraction

$$
\eta_{\mathrm{EC}}=\frac{\mathrm{P}_{L}}{\mathrm{P}_{\mathrm{EC}}}=\eta_{\mathrm{PF}} \eta_{\mathrm{EB}} \eta_{\mathrm{OP}} \eta_{\mathrm{Q}} \eta_{\mathrm{EX}} .
$$

The flow conditioning efficiency is the ratio of the laser radiation produced to the electrical power required to circulate the gas, to remove the entropy and waste heat, and to chemically regenerate the gas if required

$$
\eta_{\mathrm{FC}}=P_{L} / P_{F C} \text {. }
$$

The overall laser system efficiency is the combination of these subsystem efficiencies

$$
\begin{aligned}
\eta_{\text {Lysiser }}=\frac{P_{0}}{P_{I: C}+P_{1 C C}}= & \frac{\left(P_{0} / P_{L}\right)}{\left(\frac{P_{L C C}}{P_{L}}\right)+\frac{P_{1: C}}{P_{L}}} \\
& =\frac{\eta_{0}}{\left(\frac{1}{\eta_{I C C}}+\frac{1}{\eta_{I C C}}\right)} .
\end{aligned}
$$

The laser system efficiency is effectively the product of the optical train efliciency and the smaller of the electrical or flow subsystem efliciencies. It is therefore quite possible for a poorly designed flow and chemical regeneration system to overwhelm the electrical losses.

The fow conditioning effieiency is often expressed in terms of the volumetric efficiency of a laser cavity (pulse energy per unit volume and per unit pressure, E). The average power leaving the lascr can be written in terms of the volumetric efficiency (usually in joules per liter-atmosphere).

$$
P_{L}=E \text { p (hQL) PRF , }
$$

where $\mathrm{p}$ is pressure, PRF is the pulse repetition frequency, and $(h, \ell, L)$ are the dimensions of the optical cavity of the kaser in the pump. Now, and optical, directions respectively. Note that ulthough E is usually expressed in joules per liter-atm, it is properly dimensionless $(E=I$ corresponds to $E=$ $100 \mathrm{~J} /$ liter-atm).

The flow conditioning power is proportional to the mass flow rate in and the stagnation temperiture of the gas $T_{0}$

$$
P_{F: C}=\xi \dot{m} C_{p} T_{0} \text {, }
$$

where $C_{p}$ is the specific heat at constant pressure and $\xi$ is the fraction of the circulating power re- quired to return the fluid to its initial state on each round trip. It is the sum of the fraction required for pumping and cooling, Epc, and for chemical regeneration, $\xi_{\mathrm{CR}}$.

$$
\xi=\xi_{\mathrm{PC}}+\xi_{\mathrm{CR}} .
$$

The mass flow rate can be written in terms of the pressure, velocity $(u)$ and ratio of specific heats $(\gamma)$ as follows:

$$
\dot{m}=\left(\frac{\gamma}{\gamma-1}\right) \frac{p u h L}{C_{p} T_{0}} .
$$

The circulating power can be written in terms of the pressure, velocity and ratio of specilic heats as

$$
\text { in } C_{p} T_{0}=\frac{\gamma}{\gamma-1} p u h L \text {. }
$$

using an approximation good for subsonic mach numbers considerably less than unity.

The most efficient flow velocity is the minimum speed allowed by the pulse repelition frequency

$$
u=r(P R F)
$$

where the flush factor $f$ is the ratio of distance flowed between pulses to the optical eavity size in the flow direction $f$. I:lush factors of $f=1,3$ to 2 are typical of subsonic gas lasers. Using these expressions, we relate the flow eonditioning efficiency to the volumetric efficiency $\mathrm{E}$ as follows

$$
\eta_{\mathrm{FC}}=\frac{\mathrm{E}}{\frac{\gamma}{\gamma-1} \mathrm{f}\left(\xi_{\mathrm{PC}}+\xi_{\mathrm{CH}}\right)} .
$$

The pumping, cooling, and chemical regeneration requirements vary from laser to laser; as an estimate, we use some typical numbers characleristic of Howing gas lasers used in other applications. To oblain high beam quality (small denstly fluctuations in the gas) a nozzle and flow screen arrangement having a substantial pressure drop appears to be necessary. For a 30-psi total pressure drop around the wind tunnel circuit a pressure ratio of three is required for the pumps. This results in a loss factor 11 for pumping and cooling of $\xi \mathrm{pc}=0.55$. The requirements of pulsed laser acoustic damping and optical medium uniformity may dictate these large 
pressure drops. If it is possible to achieve a 5-psi pressure drop typical of an ordinary subsonic wind tunnel, the loss factor is reduced substantially to $\xi \mathrm{PC}$ $=0.20$. Lasers operating at low total pressure will have the advantages of lower mass flow rates and lower pumping power requirements, but they will suffer grester temperature rises during the pulse.

We are now in a position to demonstrate the relationship of the overall laser system efficiency to the optical, clectrical, and gas flow subsystem efficiencies. The gas conditioning efficiency is expressed in terms of the volumetric efficiency $E$. Figure 2-6 shows the overall laser system efriciency as a function of the electrical subsystem effieiency with the volumetric efficiency as a parameter.

For example, imagine a visible laser system with an electrical efficiency of $5 \%$ and $E=0.10(10 \mathrm{~J} \mathrm{per}$ liter-atm). This results in an overall efficiency of $3.6 \%$. If the volumetric efficiency is $3 \mathrm{~J}$ per liter-atm, the overall efficiency drop: to $2.2 \%$, a factor of two lower than if we consider only the electrical efficiency. As a second example, a multiline $\mathrm{CO}_{2}$ laser system might have an electrical efficiency of $6.3 \%$ and a volumetric efficiency of $8.5 \mathrm{~J} /$ titer-atm. corresponding to an overall efficiency of $3.8 \%$. The overall efficiency is the proper number to use in fusion reactor calculations. There is clearly a strong incentive to design efficient low-pressure-drop now systems, or better yet. to find laser media with extremuly high volumetric efficiency.

\section{Scaling Analysis of Photolytic \\ Group VI-A and Iodine Fusion Lascers}

A completely suitable high-average-power gas laser for fusion power plants has not yet been identified. Deficiencies are apparent in all concepts, usually manifested in insufficient overall efficiency rather than scalability to high pulse energy or average power. Although fusion gas laser technology is still young, we believe it is not premalure-using the most promising shortwavelength gas lasers-lo begin the problemfinding process that accompanies the conceptual design of full-scale systems, 12 We have Jaunched such an investigation, with the following objectives:

- To construct a simple systems model of a high-average-power gas laser system 1400 to 1000 kJ, I to $10 \mathrm{pps}$ (pulses per second)], which can be used to compare candidate lasers on a consistent and realistic basis.

- To define laser subsystem requirements, including electrical power conditioning, gas conditioning. chemical regeneration, and optical interfacing with a reactor.

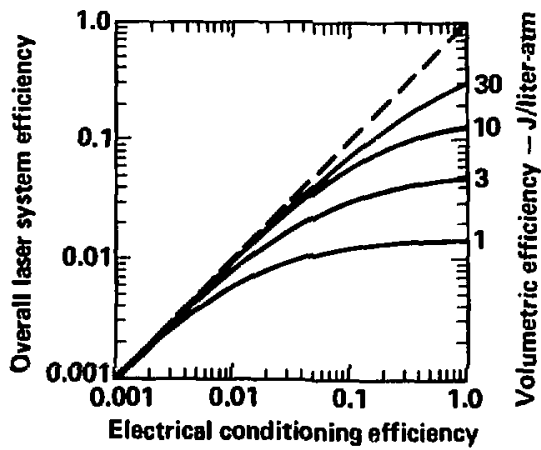

Fig. 2-6. Overall Iaser system emieiency: $\eta_{0}=0.95, t=1.2 .6$ $=0.20$.

- To perform initial system performance and cost tadeoffs within the laser system and in conjunction with the reactor system.

To identify key technical issues for advanced gas laser research and development.

To fulfill these objectives, we begin with the generic class of fluorescer-pumped photolytic lasers. We assert that, as a class, they adequately present the pulsed power, E-beam, gas flow, chemical regeneration, optical quality, and damage problems characteristic of many high-average-power fusion laser systems, thus allowing assessment of the applicability of this technology to commercial power generation systems. $\wedge$ companion study is being performed for laser-pumped photolytic lasers.

The laser medium chosen for the first comparison sludy consisis of atomic species radiating on electronic Iransitions. These excited atoms are formed by photolytic dissociation from the parent molecule which is carricd in a rare-gas buffer. ${ }^{13,14}$ The four atoms of interest are oxygen, sulfur, selenium, and iodine. The source of photolysis photons is fuorescence from an E-beam-excited rare gas, possibly doped with another molecule to enhance nuorescence radiation in the absorption band of the molecule to be photolyzed.

Table 2-3 lists the features of photolytic lasers that recommend them for fusion power plants. These features include favorable target coupling characteristies, high energy storage per aperture, promising overall efficiency, and a choice of lasing atom and pump molecule combinations.

Figure 2-7 shows one possible configuration of a high-iverag: ower photolytic luser system that appears very scalable to large volumes and high energy 
Table 2-3. Features of photolytic lasers.

1. Choice of systern:

\begin{tabular}{|c|c|c|c|c|}
\hline $\begin{array}{l}\text { Laxing } \\
\text { stom }\end{array}$ & $\begin{array}{l}\text { Lasing } \\
\text { wrvelength, nm }\end{array}$ & $\begin{array}{c}\text { Parent } \\
\text { molecule }\end{array}$ & $\begin{array}{l}\text { Fluorescer } \\
\text { excimer }\end{array}$ & $\begin{array}{c}\text { Fluorescer } \\
\text { wavelength, nm }\end{array}$ \\
\hline Iodine & 1130 & $C_{3} F_{7} 1$ & $\mathbf{X e B r} *$ & 280 \\
\hline Dxygen & 558 & & $\mathbf{A r _ { 2 }}$ & 130 \\
\hline Sulfor & 772 & $\cos$ & $\mathrm{Kr}_{2}^{*}$ & 150 \\
\hline Selenium & 489 & cose & $\mathrm{Xe}_{2}^{*}$ & 170 \\
\hline
\end{tabular}

2. Favonable tuget coupling chancteristics:

- Wretength: $\lambda=0.5=1.3 \mathrm{~mm}$

- Short pulke duration: $r \sim 1-10$ ns

3. Highenergy storage:

- $F_{\text {saturation }} \sim 1-10 \mathrm{~J} / \mathrm{cm}^{2}$

4. Favonble sceling characteristics:

- Configurations allow flowing, high-ave:gge-power systems.

- Can use low-pressure ( I atm), low-temperature ( 300 K), low-index gases (c.g., helium) for good bean quality.

- Beam quality and gain uniformity limiled by gas density uniformity, not by e-beam deposition unifurmity.

- Aperture sceling limiled by materials and gain constraints rather than by properties of the atoms and molecules.

5. EfTiciency $>1 \%$ possible from the physics.

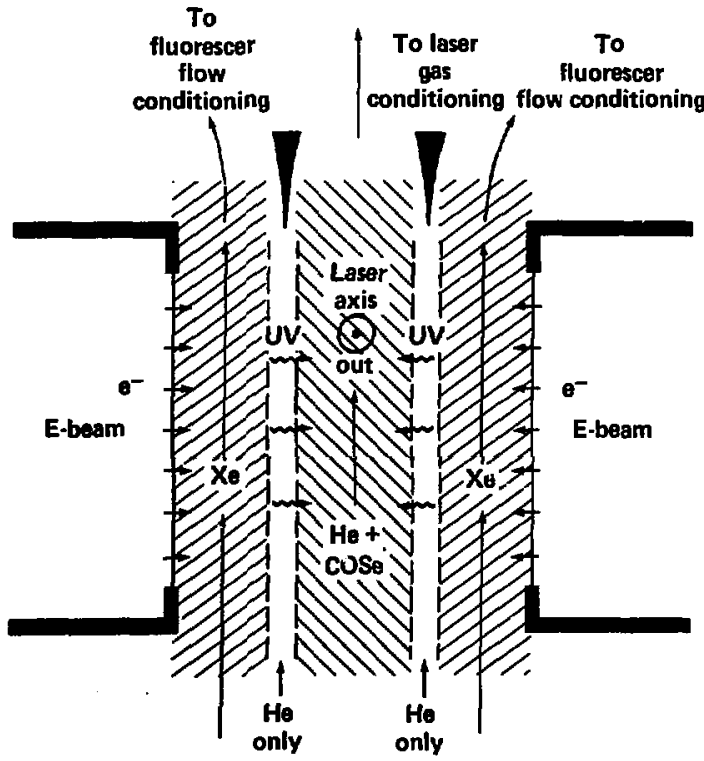

End view
Fig. 2-7. Flowlog widentess geonctry. 
per pulse. The fluorescer gas and laser gas regions are kept apart by the stability of coflowing streams of different gases. The pressure and velocity of the two gases are matched; the density, temperature, and Mach number differ. The laser does not need uv transmitting windows-a tremendous advantage since such windows probably would not be able to withstand damage from high fluence levels of photons and $1-\mathrm{MeV}$ electrons in addition to the steady and shock pressure loads over the large spans (greater than $30 \mathrm{~cm}$ ).

The configuration chosen is not the only possible phololytic laser. If a window can be found that allows operation of the fuorescer gas at a significantly higher pressure than the laser gas, or if an efficient UV laser pumped scheme can be devised, higher overall efficiencies may result. The flowing windowless fluorescer pumped geometry was chosen for this first study because we have substantial information on fluorescer efficiency but not on rare gas laser efficiencies. Furthermore, the configuration is demonstrably scalable to high average power. Comparisons between calculated efficiencies for different laser atoms will be more important than the absolute levels. Additional improvements in efficiency will no doubt come through future innovation in pumping configuration.

The lasing sequence is begun by the firing of two 0 - posed l-MeV E-beams for approximately $1 \mu \mathrm{s}$. In the case of the selenium laser sketched in Fig. 2-7, electrons radiolyze the xenon, resulting in copious xenon excimer $\left(\mathrm{Xe}_{2}\right)$ nuorescence radiation. The lasing medium consists of approximately $3 \times 10^{16}$ $\mathrm{cm}^{-3}$ of COSe in $1 \mathrm{~atm}$ of helium buffer gas, which provides a pressure mateh to the fluorescer and provides sufficient heat capacity to minimize the increase in gas temperature. The fluorescence radiation dissociates the parent molecule; the resulting excited $\mathrm{Se}^{*}$ atom stores the energy for $10^{2}-10^{3} \mathrm{~ns}$.

The molecular absorber is optically thick to the fluorescence radiation. The photolysis occurs by a bleaching wave that is driven into the medium, dissociating the molecule and causing the mixture to become transparent. The sequence ends with injection of a 1-ns laser pulse that depopulates the medium during amplification. The laser systems using $0^{*}, S^{*}$, and $I^{*}$ operate similarly.

Note that the configuration shown in Fig. 2-7 is a classic scalable geometry with the pump, flow, and optical extraction axes mutually orthogonal. This is a convenient way to decouple these important processes and reach the largest possible sizes based solely on constraints of the individual processes.

Eliciency. Figure 2-8 is a power flow diagram of a photolytic laser system. Electrical power is taken continuously from the power plant's generating system and dissipated by the electrical power conditioning ( $\left.P_{\mathrm{EC}}\right)$, the fluorescer gas conditioning ( $\left.P_{F G C}\right)$, and the laser gas conditioning $\left(P_{\text {LGC }}\right)$. The laser system output is the laser radiation focusable on target after the optical train $P_{0}$. The overall laser system efficiency is then:

$$
\eta_{L S}=\frac{P_{0}}{P_{E C}+P_{F G C}+P_{1, G C}},
$$

which can be written as in the previous section

$$
\eta_{\mathrm{LS}}=\frac{\eta_{0}}{\left(\frac{1}{\eta_{\mathrm{EC}}}+\frac{1}{\eta_{\mathrm{FC}}}\right)}
$$

The optical system efficiency is simply the ratio of the power on target divided by that emerging from the final laser aperture, which takes into account the losses in the optical transport system required to get the beam into the reactor building and focused on target. These losses include absorption and scattering of lenses and mirrors, as well as beam-shaping and diffraction-spillage losses. A typical optical train might require ten optical elements. $A s$ in the previous general analysis, we have assigned a value of $95 \%$ to this optical efficiency.

The electricat system etficiency is the tatio of laser radiation produced to electrical power provided to the pulse-forming network. It is the product of the pulse-forming network, E-beam, fluorescer, coupling geometry, quantum, and extraction efficiencies, as follows:

$$
\eta_{\mathrm{EC}}=\eta_{P F} \eta_{E B} \eta_{F} \eta_{c} \eta_{Q} \eta_{E X}
$$

In Table 2-4, these efficiencies are estimated for the four candidate photolytic lasers. A range of values is given for each laser; the lower figure represents today's technology whereas the upper figure is the goal: a reasonable number believed achievable with future development. The larger number in a sense represents the best that is envisioned for the configuration assumed in Fig. 2.7; further innovation would be needed to improve these estimates substantially.

The efficiency of the optical pump is the product of the intrinsic fluorescence efficiency of the gas, $\eta_{F}$ (40 to $50 \%$ for the rare gases, $15 \%$ for $\mathrm{XeBr}$ in Ar) ${ }^{15-17}$ and a geometrical factor, $\eta_{C}$. The latter accounts for the loss of fluorescence radiation not directed at the laser medium (15 to $40 \%$ ). The extraction efficiency is determined from the level degeneracies and the fill factor. 


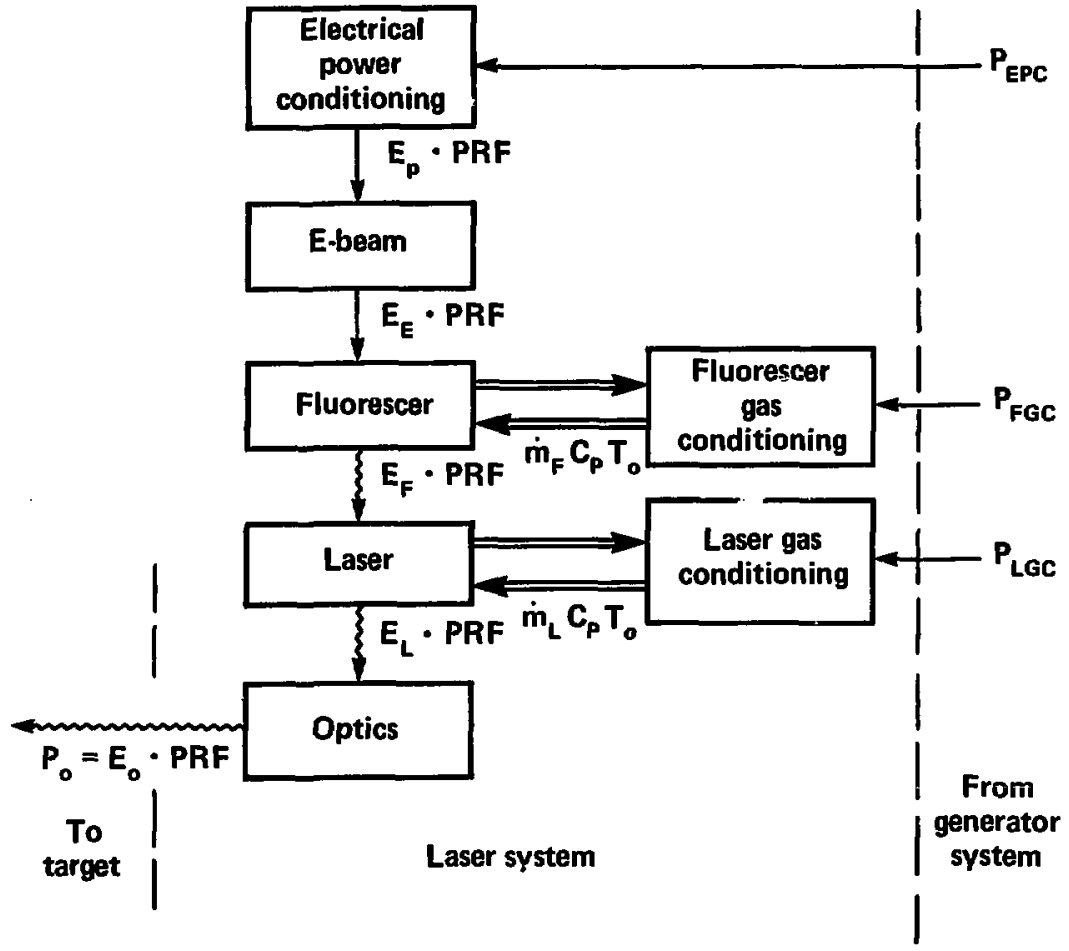

Fig. 2-8. Fluorescer pumped laser power flow.

Table 2-4. Electrical to laser efficiencies for photolytic lasers.

\begin{tabular}{|c|c|c|c|c|c|}
\hline \multirow[b]{2}{*}{ Puraneter } & & \multicolumn{4}{|c|}{ Efficiency, \% } \\
\hline & & Oxygen & Sulfur & Selenium & lodine \\
\hline Pulse forming & $\eta_{\mathrm{PF}}$ & 80.90 & 80.90 & $80-90$ & $80-90$ \\
\hline $\begin{array}{l}\text { Ebeam dlode and } \\
\text { foil holder }\end{array}$ & $\eta_{\mathrm{EB}}$ & $75 \cdot 80$ & $75-80$ & $75-80$ & $75 \cdot 80$ \\
\hline Intrinsic fluorescence & $\eta_{\mathrm{F}}$ & $40-50$ & $40-50$ & $40-50$ & 10.15 \\
\hline $\begin{array}{l}\text { Fuorescence geometric } \\
\text { coupling }\end{array}$ & $n_{c}$ & $15-40$ & $15-40$ & $15-40$ & $15-40$ \\
\hline Qunntum efficiency & ${ }^{n} \mathbf{Q}$ & 20 & 19 & 35 & 21 \\
\hline Extrection efficiency & $n_{\mathrm{EX}}$ & 83 & 83 & 75 & 67 \\
\hline $\begin{array}{c}\text { Total electrical to } \\
\text { luser efficiency }\end{array}$ & $n_{\mathrm{EC}}$ & $0.6-2.4$ & $0.6-2.4$ & $0.95-3.8$ & $0.13-0.61$ \\
\hline
\end{tabular}


Two conclusions are immediately apparent from Table 2-4. The selenium laser is electrically superior because its quantum efficiency is $50 \%$ higher than that of the other Group VI atoms. This is due, in turn, to operation on the transauroral line rather than the auroral line, which is not likely in thc oxygen or sulfur systems because of the greater gain of the auroral transitions in these atoms. The sulfur laser might alsn be operated on the transauroral line for higher quantum efficiency, but the scaling is then limited by the fact that the amplifier must be sized to compensate for the gain on the auroral line, which is 15 times greater. The iodine laser seems inferior for two reasons. First, the match between the $\mathrm{XeBr}^{*}$ fluorescence band and the $\mathrm{C}_{3} \mathrm{~F}_{7} \mathrm{I}$ absorption band is not nearly so close as for the Group VI atoms, resulting in two to three times lower fluorescence efficiency. Second, the quantum and extraction efficiencies are not as favorable. For example, the degeneracies in the iodine levels at best allow for only $67 \%$ extraction of the inverted population compared with 75 to $83 \%$ for the Group VI atoms.

The flow system efficiency is simply

$$
\eta_{\mathrm{FC}}=\frac{\mathrm{P}_{\mathrm{L}}}{\mathrm{P}_{\mathrm{FGC}}+\mathrm{P}_{\mathrm{LGC}}} .
$$

The required fluorescence and laser gas flow conditioning power are of two kinds. The first is the pumping power needed to make up stagnation pressure losses (entropy generation) due to screens and flow straighteners, friction in the heat exchangers required to cool the flow, the laser waste heat addition, acoustic absorbers, and the like. This loss is proportional to the circulating flow power. The second power is that needed for chemically regenerating the parent molecule dissociated in the laser process. This is proportional to the number density of molecules and is otherwise very dependent on details of the chemical engineering. Because our estimates of these losses for $\mathrm{N}_{2} \mathrm{O}, \mathrm{COS}, \mathrm{COSe}$, $\mathrm{XeBr}$, and $\mathrm{C}_{3} \mathrm{~F}_{7} \mathrm{I}$ are not yet firm, and because the recombination fraction is stij] uncertain, we omil these losses from this analysis. They may overwhelm the pumping power losses, particularly for the iodine laser, even though the parent molecule density is only $10^{16}$ to $10^{17}$ per $\mathrm{cm}^{3}$.

Generalizing the flow cfficiency expressions from the previous analysis to two streams, we obtain

$$
\eta_{\mathrm{FC}}=\frac{1}{\frac{2 \gamma}{\gamma-1}\left(\frac{\xi_{F} \mathrm{~W}_{\mathrm{F}}}{\mathrm{W}_{\mathrm{L}}}+\xi_{\mathrm{L}}\right)\left(\frac{\mathrm{p}}{n_{\mathrm{ex}} \mathrm{h}_{\mathrm{L}} \mathrm{N}^{*}}\right)},
$$

where $\mathrm{p}$ is the gas pressure, $\gamma$ the ratio of specific heats, $W_{F}$ and $W_{L}$ the widths of the fluorescer and laser streams, $h v_{L} N^{*}$ is the energy stored per unit yolume in excited states and, as before, $\eta_{\mathrm{ex}}$ is the fraction of the stored energy that can be extracted.

The flow efficiency depends on the relative sizes and pressure drops of the two streams and on the ratio of overall pressure to excited-state density (more precisely, on the ratio of transitional energy density to the extractable energy density of excined states). Obviously, it is desirable to operate at low helium pressure and high lasing molecule density. Note that the flow efficiency is independent of cavity volume or pulse repetition frequency, if the laser is operated at the minimum mass flow.

The flow system efficiency calculation depends on the results of scaling relationships such as the relative widths of the laser and fluorescer streams, the pressure ( 1 atm), and the parent molecule number density. However, in order to keep the discussicis of efficiency self contained, we give the results of the flow efficiency calculations in Table 2-5 for the four lasers of interest, exclusive of any chemical regeneration losses. The electrical and optical efficiency losses are repeated in this table in order to display the overall efficiency.

We immediately note that the flow efficiencies are so poor for this configuration that they significantly influence the overall efficiencies. Because the fluorescer flow and laser flow must be pressurematched in a windowless system, one must operate at high enough total pressures (and therefore mass flows) to stop the E-beam efficiently in the fluorescer flow region. This makes it difficult to reduce the total pressure much below the assumed value of $1 \mathrm{~atm}$. In addition, the oxygen and iodine lasers use argon, a relatively low-atomic-weight fluorescer which requires a much greater width of gas to stop the electrons, and therefore have a concomitant larger pumping power requirement. Other configurations, perhaps using windows and lowpressure laser gases, would have higher flow and overall efficiencies.

Scalability. Four factors dominate the sizing of photclytic lasers: parasitics, beam quality, E-beam foil damage, and the decay time of the population inversion. We have considered these factors in an analysis of the feasibility of constructing $100-\mathrm{kJ}$ pulse amplifiers. The results are encouraging; it appears that it will be less difficult to obtain size than efficiency.

Parasitic oscillations must be suppressed in large laser amplifiers to prevent unwanted premature depopulation of the excited states. In the laser direction, it is quite likely that saturable absorbers will be used to isolate amplifier stages and prevent target 
Table 2-5. Overall laser system efficiencies for windowless fluorescence-pumped photolytic lasers.

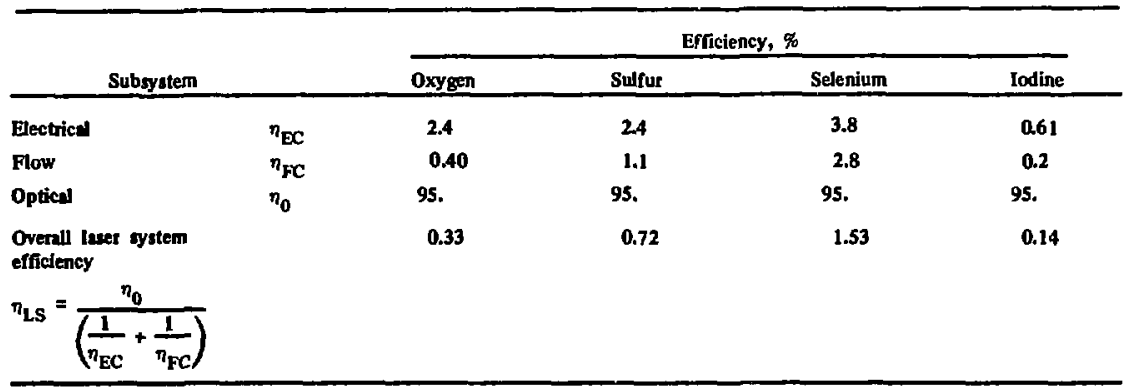

preheating. This allows the use of high gain lengthproducts, particularly if aerowindows rather than solid windows are used, for they eliminate the backreflecting problem. Consequently, we use the product of the small signal-gain and length, $\mathrm{BO}_{\mathrm{L}}=6$ as the parasitic limit on maximum length. This is a large but not unreasonable limit.

Parasitic oscillations must also be prevented in the transverse directions, particularly in the high gain fluorescer region. The flow direction is bounded in the upstream direction by a nozzle wall that may reflect light. However, the downstream direction is bounded by an aerodynamic diffuser full of hot absorbing gas, so we would anticipate no reflected radiation. In the pump direction, the laser cavity is bounded by fiuorescent gas regions, which may or may not be transparent to the laser radiation depending on the jon density in the fluorescing gas. If they are transparent, the laser radiation could reflect off the E-beam foils, which have some reflectivity in the visible spectrum. We use a parasitic limit of $\mathrm{g}_{0} \mathrm{~W}_{\mathrm{L}}=3$ to be conservative, where $W_{L}$ is the width of the active laser gas in the pump direction.

There are other limitations on the width in the pump direction, such as quenching of the excited states of the photolyzed gas by the parent molecule during the pump pulse. This limit can be overcome by pumping harder for shorter times, provided twophoton absorption does not set in. One pumping constraint is the E-beam foil damage $\left(F_{E}\right)$ limit of $20 \mathrm{~J} / \mathrm{cm}^{2}$, corresponding to $20 \mathrm{~A} / \mathrm{cm}^{2}$ for $1 \mu \mathrm{s}$ or $200 \mathrm{~A} / \mathrm{cm}^{2}$ for $0.1 \mu \mathrm{s}$ at electron energies of about 1 $\mathrm{MeV}$. The bleaching-wave velocity is sinply the fluorescent photon number flux divided by the absorber (parent molecule) density. With an E-beam fluence limit of $20 \mathrm{~J} / \mathrm{cm}^{2}$, a bleaching wave can be driven $>60 \mathrm{~cm}$ into a gas with $3 \times 10^{10}$ absorbers per $\mathrm{cm}^{3}$. This result is relatively independent of the pumping time, which can be chosen to minimize quenching problems.

Mirror damage limits are not really of concern in this example because there is enough freedom to choose an input fluence such that high efficiency is achieved while maintaining an output fluence less than the mirror threshold.

Beam quality is an important constraint for large lasers. The chief sources of phase degradation and angular spread for the coherent beam are the surface finish and thermal distortion of mirrors and density fluctuations in the gas. The latter cause nonrandom linear fluctuations in the index of refraction.

Each optical component is allowed part of an overall distortion budget, calculated from the greatest beam-quality loss that can be tolerated while still focusing on a fusion target. As an example, we performed the following linearized analysis. Assume that the optical train needed for interfacing with the fusion reactor is composed of ten mirrors, the last of which is a 2-m-diam focusing misror. ${ }^{18}$ This final mirror might be located $100 \mathrm{~m}$ from the center of the target chamber to minimize the chamber's open wall area, through which neutrons can escape. The beam is focused on a $0.5-$ to $1.0-\mathrm{mm}$ diam target.

The gas laser amplifier is assumed to have an input beam of zero phase variance and a minimum local gas density variation level $(\Delta \rho / \rho)$ due to flow noise of $10^{-3}$. This level is the least amount yet achieved in a laser cavity, although $5 \times 10^{-4} \mathrm{might}$ be reachable. Helium is used as the buffer gas because argon produces 7.5 times more fluctuation in the index of refaction than helium at the same 
average density and density-fluctuation level. It appears that 5 to 10 meter-atmospheres of laser gas can be tolerated from these considerations.

The length, height, width, and number density considerations may be combined to calculate the maximum storable energy per amplifier module; i.e., in a single aperture (Fig. 2-9). Oxygen, the system with the lowest gain, stores the greatest energy because oxygen systems-limited in both flow length and laser length by gain constraints-store more energy per module by reducing the lasing molecule density and increasing the volume by a proportionately greater amount. In fact, only the oxygen system can store more energy than can be extracted, because of a mirror-damage fluence threshold of $10 \mathrm{~J} / \mathrm{cm}^{2}$. Consequently, for the oxygen-system curve in Fig. 2-9, only number densities less than about $8 \times 10^{16}$ represent extractable energies.

Conclusion of Photolytic Group IV Scaling Analysis. Figure 2-10 presents a graphic summary of results of this initial study of high-average-power photolytic gas lasers. Amplifiers can be built large enough to achieve $1 \mathrm{MJ}$ with less than a dozen beams. Obtaining adequate efficiency will be the most difficult task; only the selenium system promises an overall efficiency above $1 \%$ for this windowless configuration. The iodine system re-

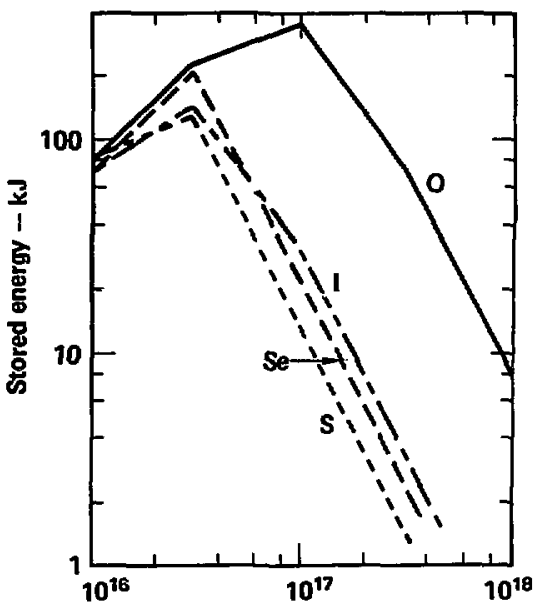

Lasing molecule density per $\mathrm{cm}^{3}$

Fig. 2-9. Maximum stored energy per aperture for photolytic laser systems. quires a tenfold higher power input than the selenium system. This could be accomplished only by operating at ten times the recirculating power or ten times the fusion pellet gain, circumstances that are either economically or physically unlikely.

This stury has been instrumental in showing that a fluorescence-pumped photolytic laser has the potential to be a full-size fusion driver. There are significant physical uncertainties remaining, and much experimental work on kinetics, gain measurements. self absorption, bleaching wave dynamics, and beam quality must be done. But we are confident that if the experiments now being undertaken bear fruit, fusion laser amplifiers of significant size $\left(\geqslant 10^{5} \mathrm{~J}\right)$ and adequate efficiency are possible.

A crucial issue is the improvement of the overall efficiency-both the electrical and flow efficiencies. The greatest leverage we have in this area is in improving the coupling of the pump raciation to the laser species, a problem amenable to some innovative throught. Excimer laser pumping is one possibility-better directionality of the pump beam will help immensely, although the excimer laser efficiencies are less thun excimer fluorescer efficien. cies for ail the rare gases. We have examined pumping geometries which use both sides of the slab of fluorescer gas, a stratagem that can raise both the electrical efficiency and the flow efficiency by nearly a factor of two. This would put the selenium laser in the $7 \%$ electrical efficiency range ( 3 to $4 \%$ overall ef ficiency range), making it a truly attractive prospect for an advanced gas laser system.

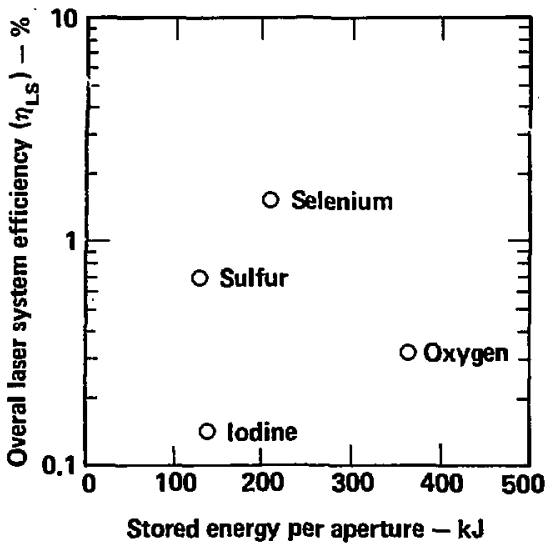

Fig. 2-10. Efficiency and scalability of plotolytic gas Iaser systems. A florescer pumped windowless geometry was used. 


\section{Reactor Concepts For Laser Fusion}

During the last year, we initialed scoping studies to identify attractive reactor concepts for producing electric power with laser fusion. These studies were carried out by developing several explosatory reactor concepts and then subjeeting them to the criteria we presented carlier for comparing long-range sources of electrical energy: abundance, social costs, technical feasibility, and economic competitiveness. The major problems affecting the technical feasibility of a laser fusion power plant werc presented earlier. In this section we explore solutions to the two major reactor vessel problems:

- The effects of high-energy neutrons and cyclic stresses on the blanket structure

- The effects of $x$ rays and debris from the fusion microexplosion on the first-wall

Our level of confidence in developing the technology to solve these problems has been determined by the amount of system development required beyond the state-of-the-art. Economic feasibility will be strongly dependent oit the solution to these technical problems: it has been assessed in terms of factors inciuding reactor size, power density, first-ivall and blanket lifetimes, duty cycle. fabrication costs, stored energy requirements, and recirculating power fractions.

The reactor concepts include:

- A liquid-lithium-cooled stainless steel :: anifold

- A gas-cooled graphite manifold

- Fluid wall concepts, such as a liquid lithium waterfall, or a ceramic-lithium pellet waterfall

Before describing these concepts, we introduce some background information on fusion reactor technology and the design parameters and constraints that are common to all of the reactor concepts. This discussion of reactor technology deals primarily with the functions that a blanket system is required to peiform and the problems associated with performing these functions in the hostile environment created by the fusion microexplosion.

All of these reactor concepts are based on an inertially confined deuterium-tritium fusion reaction. We have selected thermonuclear yields ranging from 400 to $4000 \mathrm{MJ}$ and pulse repetition rates from 1 to $10 \mathrm{~Hz}$. Selected combinations of these parameters result in reactor systems that produce $40 \%$ to $4000 \mathrm{MW}$ of thermal power and 120 to $1530 \mathrm{MW}$ of electrical power with net efficiencies ranging from 30 to $40 \%$. The variance in net effidiency results from the different thermal efficiencies and recirculating power requirements of the various concepts. At fuel pellet yields of 400 to 4000
MJ, a fuel cycle cost of 3 mills $/ \mathrm{kWh}$ will allow fiel pellet fabrication costs ranging from $12 \mathrm{e}$ to $\$ 1.20$. The selected parameter space for fusion neutron jlux at the first wall ranges from 1 to $10 \mathrm{MW} / \mathrm{m}^{2}$. resulting in first wall radii ranging from 1.5 to $15 \mathrm{~m}$.

The effects of neutrons, $x$ rays, and debris from the thermonuclear microexplosion represent the primary technical concerns that must be dealt with in laser fusion reactor concepts. All of our reactor concepts employ large focal length optics to mitigate the damaging effects to the final focusing elements. At a focal length of $10 \mathrm{~m}$, the final optics would survive the microexplosion. but they may have to be replaced at relatively short intervals. At $\mathrm{r}=100 \mathrm{~m}$ the damaging effects are reduced by two orders of magnitude, thus assuring the survival of the final focusing elements for intervals that are long enough not to adversely affect the plant availability factor. High-energy neutrons also damage and activate most structural materials. The large amounts of low-level ridioactive waste thus generated represent a maintenance and disposal problem that is common to all types of DT fusion systems. $X$-ray and debris damage to first wall materials is a problem primarily associated with inertial cờnfinement fusion systems.

Several different approaches to the first wall prohlem have been discussed in the literature, including use of a dry wall, ${ }^{19}$ wetted wall, ${ }^{20,21}$ and magnctically protected wall. ${ }^{22}$ These approaches differ primarily in the way in which the inner surface of the first wall interacts with the $x$ rays and microexplosion debris. In the dry wall approach a sacrificial metal or ceramic liner is placed between the fusion chamber and the blanket. The wet wall concepts feature a thin layer of liquid metal that covers the metal wall and protects it from the blistering and structural ablation that would otherwise occur. The magnetic protection concept uses a solenoid to divert the pellet debris away from the sides of a cylindrical blanket and into conical collectors at the top and bottom.

The fluid wall concept is a new and promising approach developed in our scoping studies. In this approach, the first structural wall is shielded from $x$ rays, neutrons, and debris by a thick waterfall of ljthium in liquid or solid pellet form. The fall contains enough moderating material to degrade the fusion neutron spectrum to the point where neutron damage levels in the structural materials are significantly reduced. These lower damage levels allow consideration of smaller blanket structures which could last for the useful lifetime of the plant.

The blanket system must perform several functions while coping with the hostile environment created by the fusion microexplosion. It must: 
- Convert the fusion energy into thermal entre!

- Provide for efficient removal of the thermal energy

- Breed enough tritium to replace that which was burned in the fusion reaction

- Maintais the required vacuum in the fusion chamber

Sixty-five to seventy-five percent of the fusion energy is in the form of high-energy neutrons. Therefore. a neutron-moderating material is required to convert kinetic energy to thermal energy. In general. elements with low atomic numbers and high scattering cross sections are effective moderators: water, hydrides, beryllium, and graphite are common examples. Although somewhat less effective. lithium can also be considered as a neutron-moderating material.

Because there is no significant natural supply of tritium, a DT fusion reactor must breed iis uwn tritium. Several neutron reactions produce tritium. but the only tritium-producing reuctions with high enough cross sections to be useful are those involving lithium. Natural lithium is $7.4 \%{ }^{6} \mathrm{Li}$ and $92.6 \%$ ${ }^{7} \mathrm{Li}$, and tritium can be produced from either isotope by:

$$
\begin{aligned}
& { }^{6} \mathrm{Li}+\mathrm{n} \rightarrow{ }^{4} \mathrm{He}+\mathrm{T}+4.8 \mathrm{MeV}, \\
& { }^{7} \mathrm{Li}+\mathrm{n} \rightarrow{ }^{4} \mathrm{He}+\mathrm{T}+\mathrm{n}-2.5 \mathrm{McV} .
\end{aligned}
$$

The ${ }^{7} \mathrm{Li}$ reaction has a threshold of approximately $4 \mathrm{MeV}$ and a much lower cross section than the ${ }^{6} \mathrm{Li}$ reaction; nevertheless, it is very important because it produces a tritiun atom without depleting the neutron population. If the neutrons are moderated before reaching the fertile lithium, the ${ }^{7} \mathrm{Li}$ reaction is not utilized (since it requires a high-energy neutron) and any leakage neutrons result in a tritium-breeding ratio less than 1.0 . In such cases, the blanket must also contain a neutron multiplier to maintain an adequate breeding ratio. Beryllium and lead with high $(n, 2 n)$ and low capture cross sections are examples of good neutron multipliers. However, beryllium is a limited resource material whose use could significantly reduce fusion's potential as a long-range source of energy.

The vacuum requirements in the fusion chamber are primarily determined by considerations of laser beam propagation and damage to the injected fuel pellet. If the DT fuel can be incorporated into the pellet in a nencryogenic or insulated form, and hence be less subject to heat dainage, laser beam propagation will be the primary factor determining the vacuum requirements in the fusion chamber. Our results ${ }^{23}$ indicate that beam defocusing and at - tenuation of 1 ant light by cascade breakdown and or thermal blooming can be reduced wa aceeptable levels with fusion thamber pressures of 0.1 Torr or less. The pumping requirements needed to maintain this vacuum will depend on the material vaporised and the type of pump used. For a lithiumwelted first wall concept, the 0.1-Torr vacuum can be maintained under the worst conditions with a vacuum pump that requires about $2 \%$ of the gross electrieal power and approximately $10 \%$ of the surface area (the worst conditions assume all the debris and $x$-ray energy is used to vaporize lithium).

\section{Liquid-I.ithium-Cooled Stainless Steel Manifold}

This concept (Fig. 2-11) represents a conventional atpproach to a laser fusion power plant, requiring less advanced technology than the fluid walt concepts. In this concept, the microexplosion is surrounded by a cylindrical annulus of stainless steel into which vertical coofant channels have been drilled to form a manifold. Liquid lithium flows down these channels and is recirculated to the top through a bulk coolant region that separates the annular manifold from an outer pressure vessel. Liquid lithium serves as priniary coolant, neutron moderator, and fertile material.

The stainless steel manifold concept is compatible with either a dry or wet first wall approach. In the

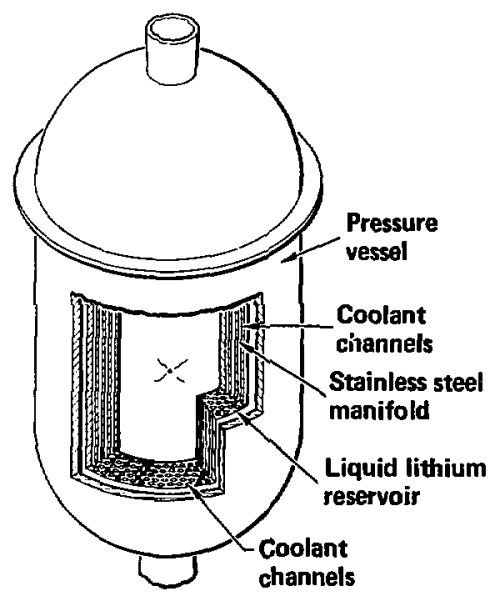

Fig. 2-11. I.iquid lithium cooled stainless steel manirold concept. 
dry wall approach, we have analyzed a graphite liner that is supported by stainless steel and cooled with liquid lithium. The graphite liner is designed for an operational lifetime of one year. In the wet wall approach, we have investigated the possibility and effects of maintaining a thin (3-mm) film of liq. uid lithium on the inner surface of the manifold.

Tritium-breding considerations limit the thickness of a solid stainless stecl structural wall to less than $10 \mathrm{~cm}$. However, it would be impossible to utilize a structural wall even this thin without internat cooling. The solid manifold design was conceived to provide additional cooling and improve the tritium-breeding performance for a given mass of structural material. In fact, we have calculated tritium-hreeding ratios greater than one for 40 -cmthick manifolds containing $50 \%$ stainless steel and $50 \%$ lithium by volume. The calculations were made using the TAKT Monte Carlo neutron transport code ${ }^{24}$ with the L.I.I.-FNDL. cross section library. ${ }^{25}$

The stainless steel manifold will operate at a neutron first wall loading of 1 to $2 \mathrm{MW} / \mathrm{m}^{2}$, and this will require a relatively large chamber radius (10 to $15 \mathrm{~m}$ for a $4000 \mathrm{MW}$, system). A major disadvantage will result if cyclic stresses and neutron damage limit the lifetime of the manifold to a few full-power years; this concept will then produce large amounts of low-level radioactive waste in the form of activated steel. It should also be noted that lithium is an element whose corrosive effects inerease with inereasing temperature. For use with stainless steel, lithium temperatures must be limited to about $500^{\circ} \mathrm{C}$. This peak temperature corresponds to a conventional steam cycle thermal efficiency of $38 \%$. 26

\section{Gas-cooled Graphite Manifold}

The graphite manifold (Fig. 2-12) is similar to the stainless steel manifold concept except that the vertical coolant channels are drilled into an array of graphite blocks that make up the fusion chamber. The vacuum vessel is an outer shell of reinforced, prestressed concrete. High-pressure helium gas is pumped through the coolant channels, some or all of which are filled with pellets of a lithium-bearing ceramic. As the tritium diffuses out of the lithium compound in which it is bred, it is removed from the channels by the gas coolant.

The graphite manifold design is a reactor concept that exhibits low activation and low tritium inventories. Moreover, the possibility of an accident occurring that could release readioactivity to the environment is greatly reduced because the lithium is present in less reactive (solid) form $\left(\mathrm{Li}_{2} \mathrm{O}, \mathrm{LiAl}_{2} \mathrm{O}\right.$. for example). The graphite moderates the neutrons below activation energy levels. It also moderates the neutrons to energies below the threshold for the ${ }^{{ }^{2} \mathbf{L i}}$ tritium-producing reaction. This makes it advanlagcous to enrich the lithium in "Li." thereby reducing the required lithium and tritium inventories. Depending on the particular design, a neutron mulliplier such as beryllium, may he required to maintain a tritium-breeding ratio greater than 1.0. The use of a gas coolant will allow high operating tcmperatures and result in high thermal conversion efficiencies.

Some problems with this concept have heen identified. The usi of beryllium to multiply neutrons and enhanec tritium-breeding presents a problem in terms of beryllium 's roxicity and relative scarcity. We arc investigating the possibility of using lead as the teutron-multiplying material. large amounts of pumping power will be required to cool the system and purge the tritium from the pellet-filled channols. Finally, the structural integrity of the graphitc chamber in the microexplosion environment may be inadequate.

\section{Fluid Wall Concepts}

Wet walls and sacrificial dry wall liners have previously been proposed as methods of protecting the metallic first wall from the soft $x$ rays and pellet debris of fusion microexplosions. The Huid wall concepts were conceived lo protect the first metallic wall from high-energy neutrons in addition to the $x$ rays and debris. We have investigated two types of

- Natural lithium contains $7.4 \%{ }^{6}$ L.i.

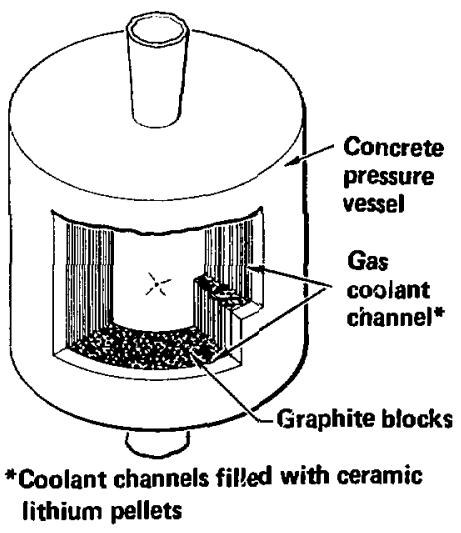

Fig. 2-12. Gas cooled graphite manifold concept. 
Fuid walls: the ceramic-lithium pellel waterliall and the liquid lahium Haterfall. Ji has been demonstrated in the nuclear fisson industry that once scientific feasibility has been achiowed. the materials development program paces the demonstration of technical and economic feasibility. Iluid uall concepts will be less dependent on materials development hecause radiation damage is signifieantly reduced. The analysis of the liquid lithium system is further simplified by the availability of data on the properties of liquid lithium and the existence of liquid-metal experimental facilities constructed in support of the I.MFBR program.

The principal feature of the ceramic-lithium pellet Aatcrfall (1-ig, 2-13) is a thick layer of falling solid ceramic-lithium pellets that shields the first struclural wall from the microexplosion. The pellets are continuously recirculated to the top of the vacuun chamber through a reservoir region betucen the first wall and the pressure vessel. The pellets are either transported through heal exchillngers or cooled by the flow of high-pressure helium gas in the reservoir region. Tritium is bred in the ceramic lithium compound and recovered as it diffuses out. Preliminary calculations indicate a tritium breeding ratio greater than 1.0 can easily be achieved

The thick region of falling pellets will moderate and absorb neutrons before they reach the first structural wall, resulting in a significant reduction in the degree of first wall damage and possibly the amount of radioactive waste produced by neutron activation. The use of lithium in a ceranic form is an imporfant feature of this concept in that it eliminates the corrosive problems of liquid lithium and significantly reduces the associated chemical hazard.

Major questions. such as iritium diffusion from the pellets and structural integrity of the ccramic compound, cannot be answered satisfactorily with existing dats. A means of efficiently transporting the pellets, particularly into and out of the vacuum chamber, is another area of major uncertainty.

The liquid lithium waterfall concept (Fig. 2-14) has emerged as an extremely promising reactor concept for a laser-fusion power plant. It features a thick continuous fall of liquid lithium that protects the first structura! wall from direct exposure to the microexplosion. The principal purpose of the fall is to reduce the neutron radiation damage in the first structural wall, allowing it to last for the useful life of the plant. Besides moderating neutrons the fall also absorbs the photons ( $x$ rays and reflected laser light), pellet debris (alpha particles, unburned fuel, and other pellet material) and any blast wave emitted by the microexplosion. Because the fall is kept

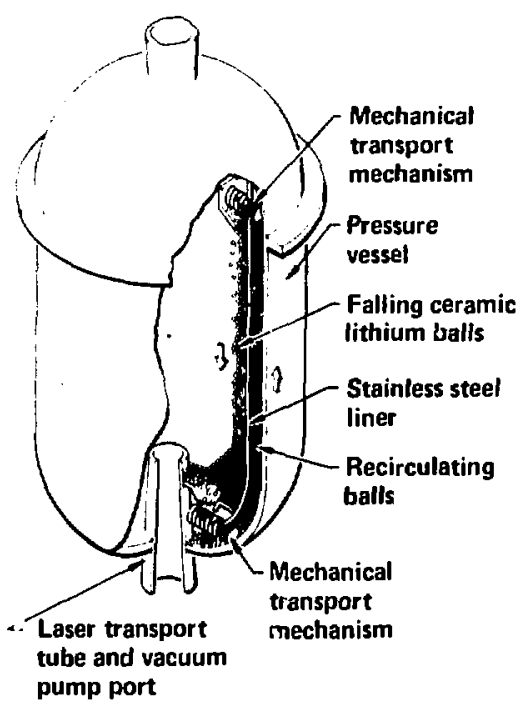

Fig. 2-13. Jalling halls concept.

off the chamber wall, any shock wave generated in the fall is not directly transmitted to the structural wall. The majority of the fusion energy is thus deposited in the liquid lithium, wbich serves as primary coolant, fertile material for tritium breeding, and first wall.

Note that fluids other than liyuid lithium could certainly be used to perform the neutron nuderating function of the falt. The primary constraints on the fall material are that the substance must:

- Have a reasonably low melting point so the fluid state can be effectively maintained.

- Have a low enough vapor pressure at the selected operating temperature $\left(>400^{\circ} \mathrm{C}\right.$ but as high as possible) to permit an adequate vacuum condition to be maintained.

- Have neutronic characteristics that permit an adequate tritium breeding ratio to be achieved.

Tritium breeding considerations preclude the use of a neutron absorber and require that lithium be incorporated in the reactor system in a suitable manner.

One possibility is to use lead us the primary constituent of the fall: the lead effectively degrades the 


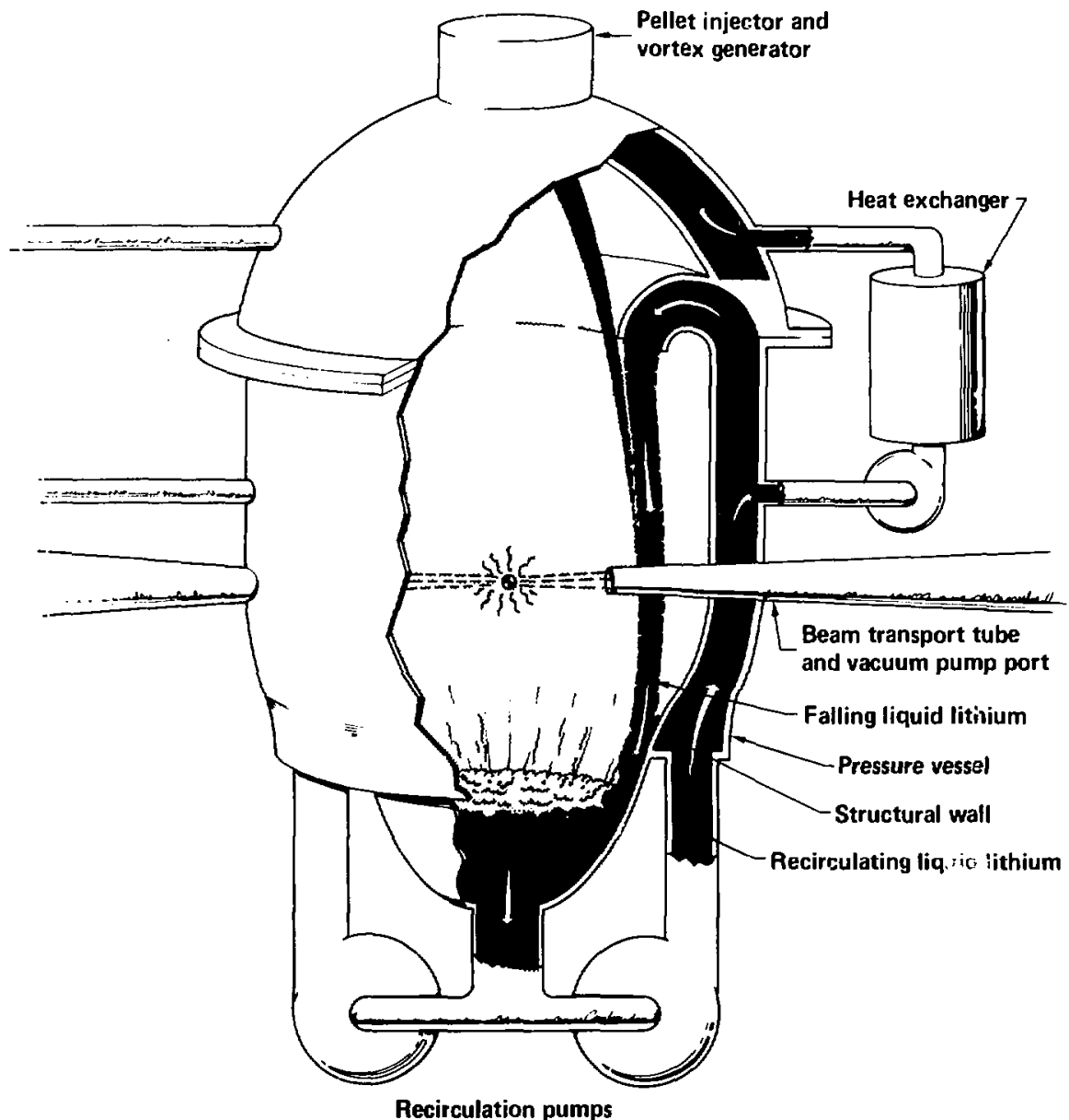

Fig. 2-14. I.iquid lithium naterfall concept.

high-energy neutron spectrum through (n, 2n) reactions and inelastic scatterings. Our TART ${ }^{24}$ calculations indicate that a few vol\% of " $\mathrm{Li}$ in the $\mathrm{Pb}$ fall would be enough to maintain a tritium breeding ratio greater than one. Alternatively, a small vol\% of lead could be added to the lithium fall to more efficiently moderate the neutron spectrum. The use of $\mathrm{Pb}-\mathrm{Li}$ alloys would decrease the system tritium inventory. On the negative side, recirculation pumping power would increase. und grain boundary corrosion of steels might present compatibility problems. The various aspects of such a system are being investigated. For the present. however, the discussion will be confined to a natural liquid lithium fall.

Laser fusion reactors have a flexible geometry that is not avitilable in magnetic confinement reactors. While a point source of energy is more effectively utilized in spherical geometry, we have selecled the cylindrical geometry shown in Fig. 2-14 
for sescral reasons. A sorted gencrater mated a sheet of lithium wes protect the un at the restion (primarily from a rays and debris). Jhes sheet is thinner than the uaterlall and dees net provide the same degree of prolection trom neutron damage. Therefore. it is advantageous to hats the lop of the thanter farther from the nicrocxplosion thats are the side walls. The spherieal end-ciep on the sylindrical chimber eliectivels atcomplishes this. I he lithium is in direct contact with the walls at the hortom of the vacuum shamber. and shock nils cin he directly transmitted (o) the chamber walls att this point. Howeser. the ehamber is well supported structurally in thes arta. furlbermers. by moving the bothom regen farther anily and decreasing the surface areit of the lithom poot at the bottom, the eflects of the directly transmitled shoch wise are reduced.

llorisomal irradiation of the latget will be roquired to taks adtanlage of long-focial-lenglh final upices. The lips of the liaser beam lubes must penetrate the wallerlall and be directly expened in the microcxpission at a dintance lest than the shamher wall radius. lortumately. lhese high damage areas represent only it minute fraction of the toral surface area (a few hundredtho of a percent) and sophisticated meisures and special materials could he used to protect them. Alernatively. sederilicial tuhe ends could be used and remotely fed inward as the tips slowly vaporize.

The attenuation of the high-cmergy meutom flux as the neutrons pass through lithium is shoun in Fig. 2-15. The ploted flux has been maltiplied by $4 \pi R^{2} 10$ eliminate the geometric attenuation fattor and generalize the curves for different radii to the lithium surfiate. These curves are also normalised to an unattenualed 1-4-MeV hux (no target. Jithium. or geometric allenuation). The three curves include the flux of all neutrons above the indicated energies. Note that $70 \mathrm{~cm}$ of lithium is sufficient to allenuate the high-energy (>10 MeV) neutron flux by nearly an order or magnitude.

First Structural Wall Lifetime. Of course, the parameter of real importance for determining the life of the first structural wall is not merely the flux but rather the damage resulting from the tempered flux. The primary neutron damage mechanisms are atomic displacements and gas production (primarily helium). Displacement damage is expressed as displacements per atom (dpa). and gas production is expressed as atom-parts-per-million (uppm). The damage limits for 316-SS at an operating temperature of $500^{\circ} \mathrm{C}$ are estimated to be 150 dpa and 500 appm helium. ${ }^{3}$ For an unprotected first wall of 316-SS, at a neutron wall loading of $1 \mathrm{MW} / \mathrm{m}^{2}$, the displacement damage rate is about 10 dpa per full

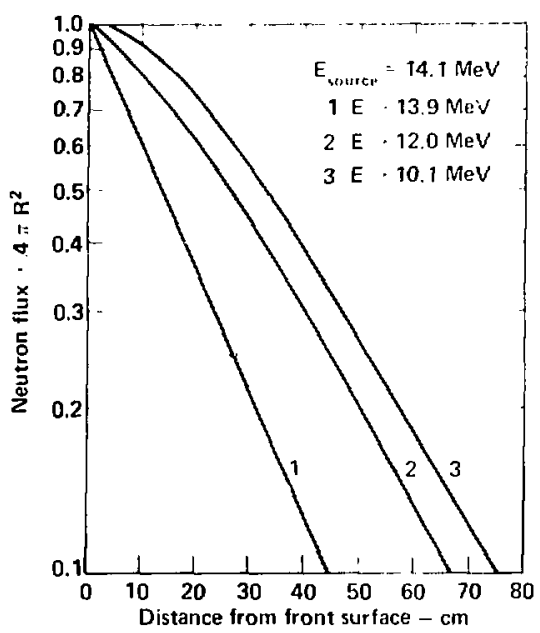

Fig. 2-15. High-encers! Hedt ron flus in dhe lithium saterfirl.

puwer sear. and the helium production rate is about 220 appm per full power sear. 'The dimape limils for He production would thus he reatehed in only 2.3 years at this wall loading.

By softening and depleting the lusion neutron speetrum. the lithium walerfill concept allows the stitinless stet vall fo remain helow the damage limits for mush longer periods of operation. teen at smaller wall racii. To make it easier lo compare our concept to others. be yuote siructural wall loadings in terms of equivalent dry wall $1.4-\mathrm{MeV}$ neutron loading. That is

$$
L=\frac{0.8 P}{4 \pi R^{2}}
$$

where

I. = wall loading. $\mathrm{MW} / \mathrm{m}^{2}$

$P:=$ thermonuclear power, MW $180^{\prime \prime}$ in neutrons)

$\mathbf{R}=$ chimber radius. $\mathrm{m}$

Of course, our actual wall toading is considerably less as a result of neutron interactions in the compressed fusion target and the lithium fall.

As seen in Fig. 2-16 the allowable first wall fluence increases exponentially with lithium thickness. Note that $40 \mathrm{~cm}$ of lithium is required to reduce helium production to the point where the 


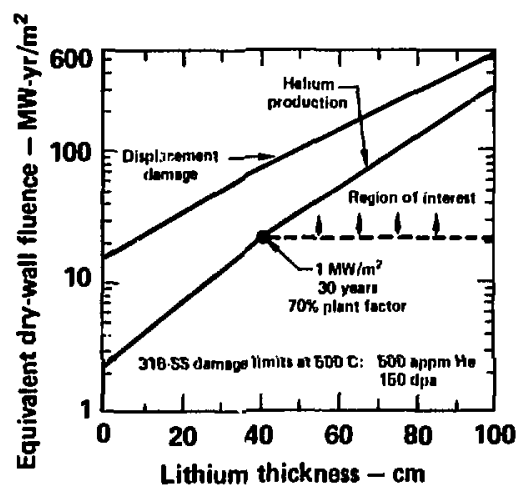

Fig. 2-16. Alluwty Fral wall Ruence as a function of lithium thicknews. The allowed fuence increases exponentially.

first structural wall could last for 30 years at I $\mathrm{MW} / \mathrm{m}^{2}$ (at $70 \%$ cupacity factor). Displacement damage is less restrictive.

We have evallatted the requirements of a system that maintidins a minimum protective lithium thickness of $60 \mathrm{~cm}$.. In determining the netutron damage to the structural first wall, we have also taken advantige of the fact that the emitted $14 \mathrm{McV}$ neutrons are attenuated by the compressed I T fuel, a characteristic unique to inertial confinement fusion. Our Montc-Carlo neutronics calculations indicatc that a fusion target with a compressed fucl Jensity-radius product, $p \mathrm{~K}$, of about $3 \mathrm{~g} / \mathrm{cm}^{2}$ is roughly equivalent to $13 \mathrm{~cm}$ of $t i$ in terms of reducing helium production: hence. the compressed target increases the effective blanket thickness to $73 \mathrm{~cm}$. Sinec heliun production dominates, the allowable fleence is $90 \mathrm{MW}-\mathrm{yr} / \mathrm{m}^{2}$. In other words, the system eould be operated at $4.3 \mathrm{MW} / \mathrm{m}^{2}$ for the 30-ycar plant life at a $70 \%$ capacity factor.

As a candidate reactor vessel for our roference power plans design, we have selected a reaces system producing $1000 \mathrm{MW}$ of thermonuclear power (700 MJ yield, I.4 pulses per second) in a cylindrital chamber with a radius of $4 \mathrm{~m}$. This corresponds to a neutron first wall loading of 4.0 $\mathrm{MW} / \mathrm{m}^{2}$ and represents a design in which the blanket structure could last for the life of the plant.

In the process of altenuating neutrons and interacting with the microcxplosion plasma the lithium fall absorbs a large fraction of the total nuclear energy deposited in the reactor. Figure 2.17 presents the cumulative energy deposition through the lithium fall and blanket region as a liuntion of the distance from the front surface of the fall. Again, a compressed larget $p \mathbf{R}$ of 3.0 has heen assumed, resulting in a neutron energy deposition in the larget of $2.2 \mathrm{MeV}$ per DT reaction. This plus the $3,5-\mathrm{MeV}$ alphi energy per DT reaction aecounts for $32 \%$ of the thermonuclear yicld which is deposited essentially at the surface of the lithium fall in the form of $x$ rays, alpha particles, and other energetic debris. The remaining fusion neutron encrgy. accounting for appoximatcly $68 \%$ of the total yield, is multiplied by 1.24. primarily as a result of exoergic tritium-producing reactions with "li:

$$
B \mathrm{Li}+\mathrm{n} \rightarrow \mathrm{T}+\alpha+4.8 \mathrm{McV} \text {. }
$$

The totil energy deposited in the reactor is 20.5 MeV per fusion reation, giving a system energy multiplicition factor* of 1.16 . As indieated, the majority of this encrgy is deposited in the (60-ent-thick lithium fall. Because lithium is the primary coolant. the system does not rely on conduction of heat through struetural materials to remove the thermal energy. I.ithiom is in fact an excellent coolant with a specific heat capacity equal to that of water and three tintes better than that of sodiumt. $A$ major advintage of absorbing all the $x$-ray and debris energy and much of the neutron energy in the coolant is lbitl the cyclicill thermal stresses in the structural walls are essentially climinated.

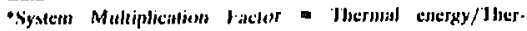
inomiclear chergy.
}

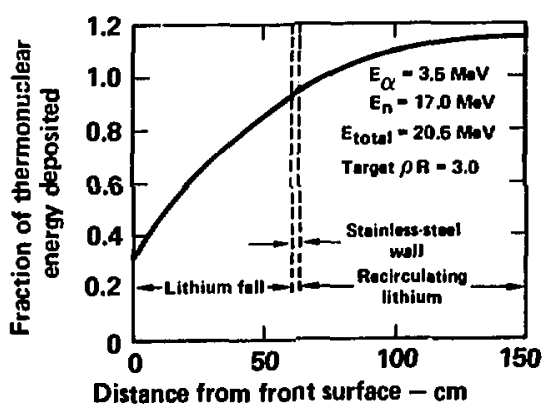

Fig. 2-17. Cimulative energy deposilion. The entrgy alsworbed is greatcl than the thermonuclear enerty becauve the newtron bsorption reaction in "I.t is exoergic. The vapor repiton betwees the fall and stalinless steel wall does not whorb much energy and, therefore, is not shown in the flpure. 
An alternative secheme proposed by the University of Wisconsin ${ }^{3}$ for the liquid lithium waterfall is to make the fall thick enough $(>80 \mathrm{~cm})$ to absorb over $90 \%$ of the total energy. In this way, the recirculating reservoir region would not be required for tritium breeding or energy removal, and the structural first wall eould be independently cooled at a lower temperature. $\wedge$ decrease in the wall tem. perature would significantly relax radiation damage limits for dpa and appm He. ${ }^{3}$ The first wall could then be operated at a higher wall loading und thus increase the power density of the reactor system. Naturally a higher power density results in a smaller reactor vessel size for a given power system; therefore, the capital cost of the reactor is reduced.

'Trilium Breeding. As one might suspect, the liquid lithium waterfall coneept has excellent tritium brecding characteristics. With no structural material between the fusion neutrons and the lithium fall, the design takes full advantage of the ${ }^{7} \mathrm{Li}\left(\mathrm{n}, n^{\prime} \mathrm{T}\right)$ reaction. The reuction only occurs with high-energy neutrons. Figure 2-18 shows the distribution of tritium bresding from ' $\mathrm{L} L \mathrm{i}$ and ${ }^{7} \mathrm{Li}$ reacetions in a $50-\mathrm{cm}$ lithium fall and $100-\mathrm{cm}$ recirculating lithium blanket region. $\wedge$ one-dimensional spherical model was used in this Monte Carlo calculation, ${ }^{24}$ and a comprosied target $\$ R$ of 3.0 $\mathrm{g} / \mathrm{cm}^{2}$ was assumed. $A$ tritium breeding ratio of 1.0 is obtained in the fall alone, and the tolal tritium breeding ratio is 1.7 . The excess Iritium produced in this reactor could in fact supply fuel for other laser fusion applications where tritium breeding is difficult or impractical. Two examples of such applications are radiolytic hydrogen production where it is desirable to deposit the neutron energy in steam rather than lithium blankets, and propulsion applicutions where weight and volume considerations art extremely important.

The total equilibrium tritiun inventory in the liqujd lithium will depend on the quantity of lithium required for the reactor system and the tritium reprocessing efficiency. The total lithium inventory is roughly estimated at 600 metric tons for the 1160 $\mathrm{MW}_{1}$ (1000 MW of thermonuclear power), 4-mradius system. At a tritium reprocessing capability of 10 appm, ihis corresponds to about $2.5 \mathrm{~kg}$ of tritium, which is equivalent to the amount of tritium injected as fuel pellets in just 4 days of operation of this system (assuming $25 \%$ pellet burn).

Chamber Vacuum. As previously stated, the vacuum condition required for laser beam propagation is on the order of 0.1 Torr. Figure 2-19 shows that the vapor pressure of lithjum is orders of magnitude less than 0.1 Torr at reasonably high

\begin{tabular}{|c|c|}
\hline \multirow[t]{2}{*}{ Lithium fall } & $\begin{array}{l}T_{6}=0.44 \\
T_{7}=0.56\end{array}$ \\
\hline & $T_{\text {fall }}=1.00$ \\
\hline \multirow[t]{2}{*}{$\begin{array}{l}\text { Recirculating lithium } \\
\text { region }\end{array}$} & $\begin{array}{l}T_{6}=0.62 \\
T_{7}=0.11\end{array}$ \\
\hline & $\begin{array}{l}T_{\text {raetre }}=0.73 \\
T_{\text {total }}=1.73\end{array}$ \\
\hline \multicolumn{2}{|c|}{$\begin{array}{l}\text { - 50.cm natural lithium fall } \\
\text { - 100.cm natural lithium "ecirculating region } \\
\text { - Target } p R=3.0 \mathrm{~g} / \mathrm{cm}^{2}\end{array}$} \\
\hline
\end{tabular}

Fig. 2-18. Trlilum brecding perfurmance.

lemperatures. Corrosion considerations require that, for use with stainless stcel, lithium temperatures must be limited to less than $500^{\circ} \mathrm{C}$. The vapor prossure al this temperature is less than $5 \times$ $10^{-3}$ Torr.

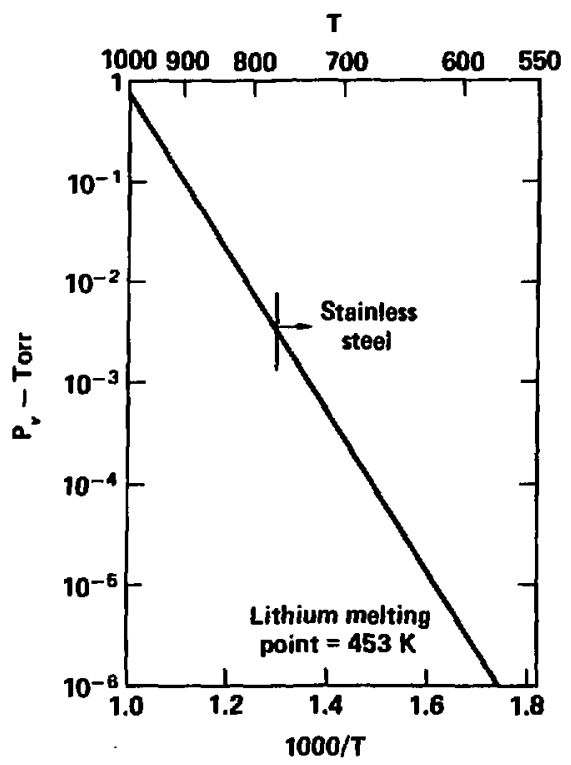

Fig. 2-19. Illhium vapor pressure vo temperature. All temperatures abown are in degrees Ketvin. The equailon plotted is P. $($ Torr $)=8.19 \times 10^{7} \exp (-18444 / \mathrm{T})$. 
Each microexplosion will vaporize a certain amount of lithium, thus increasing the chamber pressure above the required 0 .1-Torr vacuum condition. The amount of lithium vaporized and the resulting chamber pressure will depend on the initial system conditions and on the way the fusion energy couples with the liquid lithium. Various portions of the fusion energy could conceivably be used to heat liquid lithium, vaporize lithium, and heat lithium vapor. If the chamber pressure equals the liquid vapor pressure prior to the microexplosion, even deposited neutron energy could vaporize lithium, The debris and $x$-ray energy, which is deposited over a very short range at the fluid surface, will effectively blow off lithium vapor.

After the initial transient events, a certain amount of lithium vapor will exist in the chamber. The amount of lithium vaporized and the resulting quasi-equilibrium pressure rise in the chamber are determined as follows:

With respect to liquid lithium at some temperature $T_{0}$ the enthalpy change as the lithium is vaporized and heated is:

$$
\Delta h=\int_{T_{0}}^{T_{v}} c_{p}(l i q) d T+h_{q_{g}}+\int_{T_{v}}^{T} c_{p} \text { (ges)dT },
$$

where

$$
\begin{aligned}
& \Delta h=\text { enthalpy change of lithium }(\mathrm{MJ} / \mathrm{kg}) \\
& T_{v}=\text { temperature at which lithium } \\
& \text { vaporizes }(K) \\
& C_{p}=\text { heat capacity }\left(M J / k_{g} \cdot K\right) \\
& h_{\mathrm{g}}=\text { heat of vaporization }(\mathrm{MJ} / \mathrm{kg}) \\
& \Delta \mathrm{m}=\operatorname{mass} \text { vaporized }(\mathrm{kg})=E / \Delta h \\
& E=\text { fraction of the fusion energy used to: } \\
& \text { (1) heat liquid lithium and vaporize it, } \\
& \text { (2) vaporize lithium without heating, }
\end{aligned}
$$

The resulting "equilibrium" chamber pressure rise is

$$
\Delta \mathrm{p}=\frac{\Delta \mathrm{mRT}}{\mathrm{MV}},
$$

where

$$
\begin{aligned}
\mathbf{R} & =8.315 \mathrm{~J} / \text { mole }-\mathrm{K} \\
\mathbf{M} & =6.941 \times 10^{-3} \mathrm{~kg} / \text { mole } \\
\mathbf{V} & =\text { chamber volume }\left(\mathrm{m}^{3}\right) \\
\Delta \mathrm{p} & =\text { pressure rise }\left(\mathrm{N} / \mathrm{m}^{2}\right) .
\end{aligned}
$$

The maximum mass will be s aporized if the liquid is initially at the vaporization temperature associated with the chamber pressure and if no energy is expended to heat lithium vapor. A condition of supersaturation would result with the gas and liquid at the same temperature:

$$
\Delta \mathrm{m}_{\max }=\frac{E}{\mathrm{~h}_{\mathrm{gg}}} \text {. }
$$

If the liquid is at the vaporization temperature corresponding to the chamber pressure and if $C_{p}$ (gas) is constant, the equilibrium pressure rise may be written in terms of the mass vaporized, $\Delta \mathrm{m}$ :

$$
\Delta p=\frac{\Delta m R}{M V}\left[T_{v}+\frac{1}{C_{p}(g a s)}\left(\frac{E}{\Delta m}-h_{l g}\right)\right]
$$

or rearranging terms

$$
\Delta p=\frac{R}{M V}\left[\frac{E}{C_{p} \text { (gas) }}-\Delta m\left(\frac{h_{\mathrm{lg}}}{C_{p} \text { (gas) }}-T_{v}\right)\right]
$$

Since $h_{1 g} / C_{p}$ (gas) $>T_{v}$, the maximum equilibrium pressure rise results if no lithium is vaporized and all the energy is used to heat the vapor already in the chamber:

$$
\Delta \mathrm{P}_{\max }=\frac{\mathrm{R}}{\mathrm{MV}}\left[\frac{\mathrm{E}}{\mathrm{C}_{\mathrm{P}(\mathrm{gas})}}\right] .
$$

If the entire fusion pulse energy heats the vapor (in our reference design-700 MJ in $400 \mathrm{~m}^{3}$ ), the maximurn equilibrium pressure rise is about $7 \mathrm{~atm}$. In the more likely case of only $x$-ray and debris energy heating the vapor, the maximum equilibrium pressure rise is about 2 atm.

Whatever the chamber pressure is after the microexplosion, it must be reduced to the required vacuum condition of 0.1 Torr before the next pulse. As will be discussed later, there is so much liquid lithium in the chamber at the time of the microexplosion that the mixed-mean temperature rise of the fall per pulse is quite small. (For example in the $4 \mathrm{~m}$ reference chamber at $4.0 \mathrm{MW} / \mathrm{m}^{2}, \Delta \mathrm{T}$ is less than $5^{\circ} \mathrm{C}$ ). The supersaturated vapor therefore recondenses on the liquid lithium in the chamber. In effect, the liquid lithium waterfall acts as a condensing vacuum pump for the chamber.

The effectiveness of the lithium liquid in condensing the vaporized lithium will depend on the condition of the fall (bulk temperature, surface temperature, whether or not it is disassembled) and on 
the condition of the vapor (temperature, pressure) shortly after the microexplosion. The liquid fall conditions are important for determining the vapor pressure of the liquid and the sticking cocfficient, defined as the probability that a gas molecule incident on the liquid surface will stick. In general the sticking coefficient must be determined experimentally with the gas at sufficiently low pressure that free molecular flow conditions exist. ${ }^{28}$ In addition, these experiments require a small cryosurface so that negligible pressure gradients exist and the temperature of the gas is equal to the temperature of the vessel wall.

These conditions, however, do not describe the lithium waterfall chamber because the "cryosurface" is, in fact, large and the vapor will exist in the continuum pressure region where molecular coljisions are very important. By general definition, continuum conditions exist when some characteristic dimension of the vacuum chumber is at least 100 times the mean-free-path of the gas molecules. For lithium gas at $700 \mathrm{~K}$ in a 4-m-radius vessel, continuum conditions exist at pressures greater than about $6 \times 10^{-3}$ Torr. $A s$ the pressure increases from the free molecular flow region to the continuum region, the pressure gradient pushes the gas at increasing speed towards the cryosurface. Because this increases the rate of collisions with the condensing surface, the pumping speed increases if the sticking coefficient does not decrease significantly. The pumping speed reaches a limiting value and levels off as the speed of the gas stream reaches the local sonic velocity at the condensing surface. This condition is analogous to choked flow through an orifice and will exist when $2 \mathrm{~B}$.

$$
\frac{p_{d}}{p_{u}}=(1-s)<\sigma_{c} .
$$

where

$$
\begin{aligned}
& p_{u}=\text { upstream pressure } \\
& p_{d}=\text { downstream pressure } \\
& S=\text { sticking coefficient } \\
& \sigma_{c}=\text { critical pressure ratio } \\
& \sigma_{c}=\left(\frac{2}{\gamma+1}\right)^{\frac{\gamma}{\gamma-T}} \\
& \gamma=\text { specific heat ratio }
\end{aligned}
$$

These expressions are for frictionless adiabatic flow, which may be a good approximation to the flow-through transition in the condensing process. ${ }^{29}$ The plateau in the pumping speed curve will thus be reached when $S>0.51$ for a monatomic ideal gas $(\gamma=1.67)$ or when $S>0.47$ for a diatomic ideal gas $(\gamma=1,40)$. The maximum pumping speed $(S=1)$ is given by ${ }^{29}$ :

$$
\dot{m}=p A\left[\frac{\gamma M}{R T}\left(\frac{2}{\gamma+1}\right)^{\frac{\gamma+1}{\gamma-1}}\right]^{1 / 2}
$$

where

$$
\begin{aligned}
& \dot{\mathbf{m}}=\text { mass removal rate, } \mathrm{kg} / \mathrm{s} \\
& \mathbf{p}=\text { chamber pressure, } \mathrm{N} / \mathrm{m}^{2} \\
& A=\text { condensing surface area, } \mathrm{m}^{2} \\
& \mathbf{M}=\text { molecular weight, } \mathrm{kg} / \text { mole } \\
& \mathbf{R}=\text { gas constant, } 8.315 \mathrm{~J} / \mathrm{mole}-\mathrm{K} \\
& \mathrm{T}=\text { gas temperature, } \mathrm{K} .
\end{aligned}
$$

As lithium vapor is condensed the density of the gas in the chamber decreases according to 21 :

$$
\frac{V d e}{d t}=-\dot{m}
$$

Using the expressions for an adiabatic process.

$$
\frac{\mathrm{p}}{\mathrm{p}_{0}}=\left(\frac{\rho}{\rho_{0}}\right)^{\gamma} \text { and } \frac{\mathrm{T}}{\mathrm{T}_{0}}=\left(\frac{\rho}{\rho_{0}}\right)^{\gamma-1}
$$

in the expression for $\dot{m}$ and integrating the differential equation gives an expression for the chamber pressure as a function of time after the microexplosion. Assuming the lithium vapor behaves as an ideal monatomic gas, we find that the pressure decays according 10:

$$
\frac{p}{p_{0}}=\left[\frac{t}{3 \tau}+I\right]^{-5} \text {. }
$$

where

$$
\tau=\frac{0.04 \mathrm{~V}}{\mathrm{AS} \sqrt{\mathrm{T}}} \text { seconds. }
$$

$P_{0}$ is the "equilibrium" chamber pressure immediately after the microexplosion but before recondensation begins. $P_{0}$ is essentially equal to $\Delta p$ 
because the background pressure before the pulse is so low.

Figure 2-20 shows the pressure decay as vaporized lithium is recondensed by the fall. These curves represent worst-case calculations in which all the fusion energy (700 MJ in a 4-m chamber) is used to vaporize lithium at $700 \mathrm{~K}$. While an increase in vapor temperature would result in a higher value of $P_{0}$, the sonic velocity of the gas would also be higher and the gas would condense more quickly. The sticking coefficient of 0.5 is pessimistically low for a liquid metal vapor. Also, if the fall is disassembled by the neutron energy deposition, the condensing

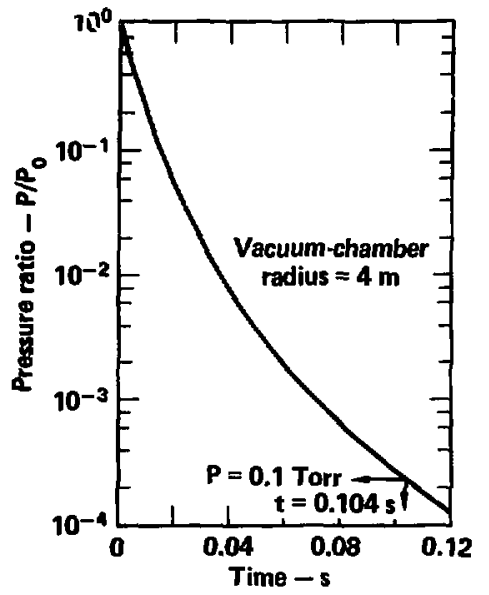

$$
\begin{aligned}
P / P_{0} & =\left[\left(\frac{8.33 \mathrm{AS} \sqrt{T}}{V}\right) t+1\right]^{-5} \\
A & =\text { condensing surface area }=\text { fall surface } \\
\text { area }-\mathrm{m}^{2} & \\
S & =\text { sticking coefficient }=0.5 \\
T & =\text { vapor temperature }=700 \mathrm{~K} \\
V & =\text { vacuum chamber volume }-\mathrm{m}^{3} \\
t= & \text { time }-\mathrm{s} \\
P_{0}= & \text { ERT } \\
E= & \text { energy used for vaporization }=700 \mathrm{MJ} \\
R= & \text { gas constant }=8.315 \mathrm{~J} / \mathrm{mole} \mathrm{K} \\
h= & \text { heat of vaporization }=19.6 \mathrm{MJ} / \mathrm{kg} \\
M= & \text { molecular weight }=6.941 \times 10^{-3} \\
& \mathrm{~kg} / \mathrm{mole}
\end{aligned}
$$

Fio. 2-20. Pressure decuy as vaporized linhium is recondensed by the fall. surface area will be much larger than the original surface area of the fall assumed here.

Even with these assumptions the vacuum conditions return to the required 0.1 Torr in about $0.1 \mathrm{~s}$ for the 4-m chamber. Thus while the exact timedependent chamber conditions have not been determined, this analysis indicates that vacuum conditions can be maintained and are in fact aided by the presence of the liquid lithium fall.

Pumping Power. We now turn our attention to what is required in terms of pumping power and flow rates to maintain the thick fluid wall of lithium. Figure 2-21 shows the model and constraints used to calculate the pumping power required to recirculate the lithium fall. The fall protects the cylindrical portion of the chamber wall, which has a height-to-diameter ratio of one. (Recall that a vortex-generated sheet of lithium protects the top hemispherical end-cap, and a pool of lithium protects the bottom). The flow inlet forms an annulus of thickness $\Delta \mathbf{R}_{0}$, with the inner edge of the ring at one-half the chamber wall radius, $R_{w}$. With this geometry, the region at the top protected just by the vortex generator represents only about $5 \%$ of the total solid angle. The fall is injected vertically downward with an inlet velocity $v_{0}$. If it is assumed that the flow maintains its original inner radius as it falls, then continuity requires that the thickness of the annulus decrease as the fluid is accelerated by gravity.

The pumping power is estimated from:

$$
\text { P.P. }=\left(1 / 2 V_{0}^{2}+g H_{0}\right) \rho V_{0} A_{0}
$$

where

$$
\begin{aligned}
H_{0}=\text { fall height }=2 R_{w} & \\
A_{0}=\text { inlet flow area } & =\pi\left[\left(1 / 2 R_{w}\right.\right. \\
& \left.\left.+\Delta R_{0}\right)^{2}-\left(1 / 2 R_{w}\right)^{2}\right] .
\end{aligned}
$$

This expression accounts only for the kinetic and static heads required for the flow. In comparison, frictional losses in the recirculating reservoir region will be small. The piping to and from the recirculating pumps will also have losses (due to friction, bends, and fittings such as valves), which are proportional to $V^{2}$, where $V$ is the fluid velocity in the pipe. The exact heat loss will depend on the type of pipe and the specific plumbing layout. Large flow area piping will allow the velocity to remain low for a given mass flow rate and help minimize these losses. 
Preliminary calculations indicate that the fall may be disassembled by the microexplosion. If this is the case, the inlet velocity must be sufficient to allow the fall to reestablish itself prior to the rext microexplosion. A clearing ratio of unity should be adequate. The first constraint is therefore:

$$
H_{0}=2 R_{w} \leqslant V_{0} \tau+1 / 2 g \tau^{2} \text {, }
$$

where

$$
1 / \tau=\text { pulse repetition rate. }
$$

As previously noted, $60 \mathrm{~cm}$ of lithium is thick enough to provide adequate protection to the first structural wall up to $4.3 \mathrm{MW} / \mathrm{m}^{2}$. Therefore, the second constraint is that source neutrons must be attenuated by at least $60 \mathrm{~cm}$ of lithium at any point through the fall.

Figure 2-22 shows how the fall thickness decreases as a function of distance from the inlet for various inlet velocities. The thicknes $q$ at any point is plotted as the fraction of the inlet thickness:

$$
\frac{\Delta R}{\Delta R_{0}}=\frac{V_{0}}{\sqrt{v_{0}^{2}+2 g H}},
$$

where $H$ is the distance from the inlet. The intersection of the dashed lines with the curves gives the distance traveled from the inlet in $0.5 \mathrm{~s}$ and $1.0 \mathrm{~s}$ and thus indicates the inlet velocity required for pulse repetition rates of $2 \mathrm{~Hz}$ and $1 \mathrm{~Hz}$, respectively, for a given required distance.

The effective attenuating thickness of the fall is $\Delta R / \cos \theta$, where $\theta$ is the angle of incidence of the source neutrons. Figure 2-23 is an example of the effective thickness for a 5-m-radius chamber, with the center of the fall at a radius of $\mathbf{3} \mathrm{m}$ and an intet velocity of $5 \mathrm{~m} / \mathrm{s}$ (as required by the first constraint for a $1-\mathrm{Hz}$ pulse rate). Note that the minimum thickness occurs very near the horizontal midplane. The exact position depends on the particular geometry and inlet velocity but is always close to the midplane. The constraint on minimum effective thickness has therefore been taken at the midplane to simplify the calculations. The second constraint is:

$$
\Delta \mathrm{R}_{\mathrm{In}} \geqslant 60 \mathrm{~cm} \text {. }
$$

When the reference 4-m-radius reactor is used as an example, the required inlet velocity at a reptition rate of $1.4 \mathrm{~Hz}$ is about $8 \mathrm{~m} / \mathrm{s}$. For $60 \mathrm{~cm}$ at the midplane, an inlet thickness of $0.9 \mathrm{~m}$ is required (inlet :rea $=14 \mathrm{~m}^{2}$ ), resulting in a pumping power of about $6 \mathrm{MW}$, which is about $1 \%$ of the gross electrical power. Other losses in the loop might double this figure but the result is still only a small percent of the gross power.

For smaller chambers the clearing ratio constraint is less important than the midplane thickness and total inlet thickness constraints. For example a 3-m-radius chamber requires an inlet velocity of only about $1 \mathrm{~m} / \mathrm{s}$. At this velocity, however, the midplane thickness is only $13 \%$ of the inlet

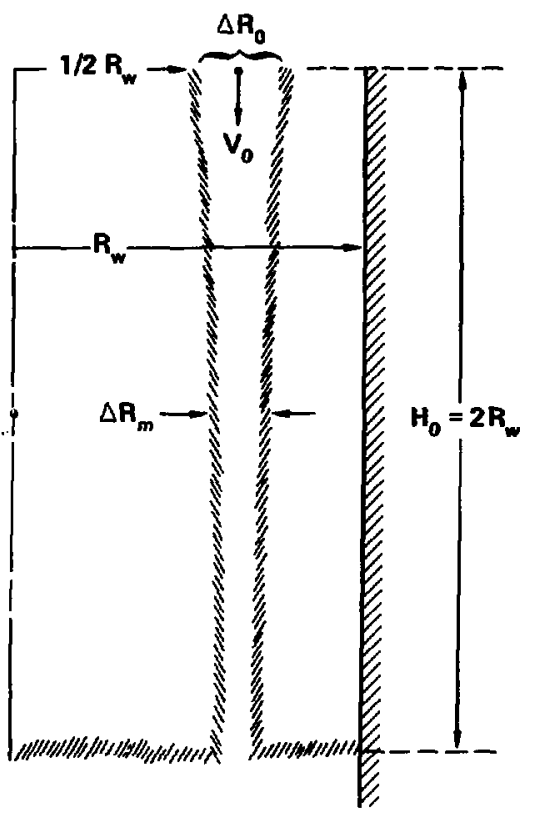

Pumping power $\simeq\left(1 / 2 \mathrm{~V}_{0}^{2}+g \mathrm{H}_{0}\right) \rho \mathrm{V}_{0} A_{0}$

Constraints:

- Fall clearing ratio of at least unity

$H_{0} \leqslant V_{0} \tau+1 / 2 g \tau^{2}$

where $1 / \tau=$ repetition rate

- Fall thickness at midplane of at least $60 \mathrm{~cm}$

$$
\Delta R_{m} \geqslant 60 \mathrm{~cm}
$$

- Inlet thickness less than $1 / 2 R_{w}$

$$
\Delta R_{0}<1 / 2 R_{w}
$$

Fig. 2-21. Recirculation pumphig power catculation-slingle fall. 


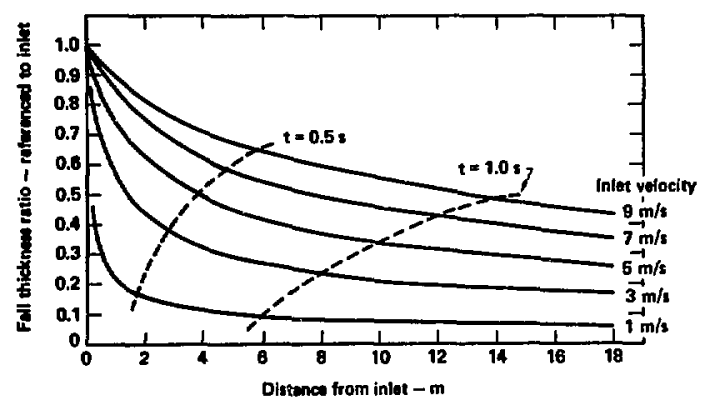

Fig. 2-22. Fall thicknes as : function of distance for various inlet velocities.

thickness, which would have to be over $4.6 \mathrm{~m}$ thick to provide $60 \mathrm{~cm}$ of protection at the midplane. Because this is larger than one-half the chamber radius, a higher inlet velocity must be used in calculating the pumping power.

The pumping power required to recirculate the lithium fall is presented in Fig. 2-24 for systems with chamber radii ranging from 4 to $10 \mathrm{~m}$. At $1 \mathrm{~Hz}$ the pumping powers are acceptably low for all chamber sizes. At the higher repetition rate the inlet velocity required to cover the length of the chamber in the $1 / 2 \mathrm{~s}$ between microexplosions is so great that the pumping power becomes quite large.

For equivalent first wall loadings the gross power produced by reactors of yarying size is proportional to $R_{w}^{\mathbf{2}}$. The fraction of the gross power required to recirculate the fall for the various sized reactors is plotted in Fig. 2-25. A gross power of $440 \mathrm{MW}_{\mathrm{e}}$ for a 4-m-radius chamber is used as the basis (1160 $M W_{\text {th }}$ at $38 \%$ thermal conversion efficiency). As indicated, at $1 \mathrm{~Hz}$ the pumping power is only a few percent of the gross electric power. At higher repetition rates the fraction becomes prohibitive for large chambers that require very high inlet velocities.

The possible advantages of using multiple falls entering the chamber at different vertical positions have also been investigated. The primary advantage is a reduction in the velocity head required to obtain a clearing ratio of one. Each fall is required to reestablish only to the inlet of the next lower fall. This is especially important at higher repetition rates. A second advantage is that the static head requirement is reduced for the fraction of the flow dclivered to the lower falls. Also, if the primary injected fall should tend to break up into separate streams instead of forming a continuous curtain, the lower falls could replenish it.
A calculation model for a system with two falls, one entering at the top as before and one at the midplane, is illustrated in Fig. 2-26. The constraints on the fall are the same as for the single-fall case, with the actual positions of minimum effective thickness used to determine the required inlet thicknesses. As before, the inlet thickness (now equal to the sum of $\Delta R_{10}$ and $\Delta R_{20}$ ) must be less than $1 / 2 R_{w}$.

The fraction of the gross power required to recirculate the double fall is shown in Fig. 2-27. Note particularly the substantial reduction in pumping power for the larger chambers at $2 \mathrm{~Hz}$ (5\% compared to $14 \%$ for the $10-\mathrm{m}$-radius chamber). The advantages of reduced velocity and static heads have been offset by larger flow area requirements for the smaller chambers at a repetition rate of $1 \mathrm{~Hz}$.

Note that the double-fall geometry is not by any means optimal. It is presented only to illustrate the possible advantages of multiple falls. Also note that these theoretical pumping power calculations do not include the efficiency of the pump or drive motor. These factors will depend on the specific design, but for large axial flow pumps the combined efficiency could be about $80 \%$. ${ }^{30}$

The flow rate required to maintain the lithium fall is plotted in Fig. 2-28. These rates are for the singlefall geometry and are based on the velocity and inlet flow area determined from the model and constraints (Fig. 2-21) previously discussed. A flow rate of about $110 \mathrm{~m}^{3} / \mathrm{s}$ is required for a 4 -m-radius reference design that operates at 1.4 pulses per second. Large-fiow-rate liquid metal pumps have been designed. Some typical parameters of a specific design supplied to us by the Liquid Metal Engineering Center are listed in Table 2-6. ${ }^{30}$ The basic stage of this design develops a 50-ft head; if higher heads are required several stages may be used in series. At 


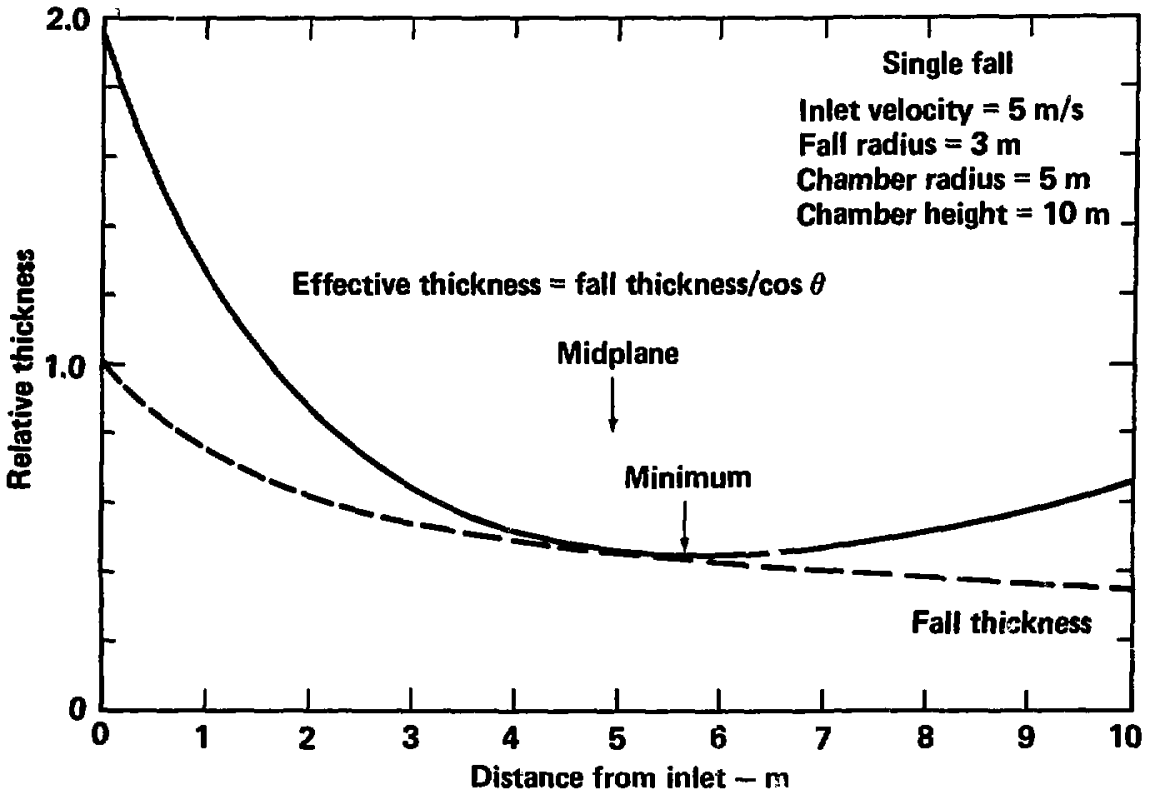

Fig. 2-23. Effective thickness of the fall. 


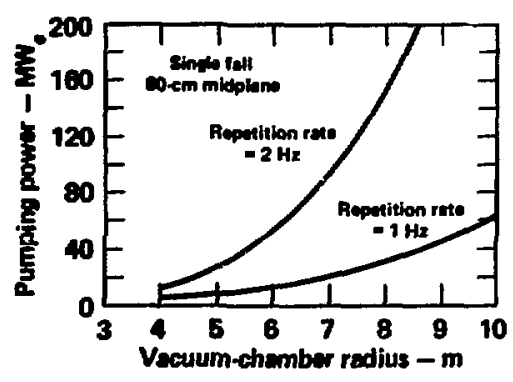

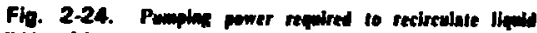
Inting ten.

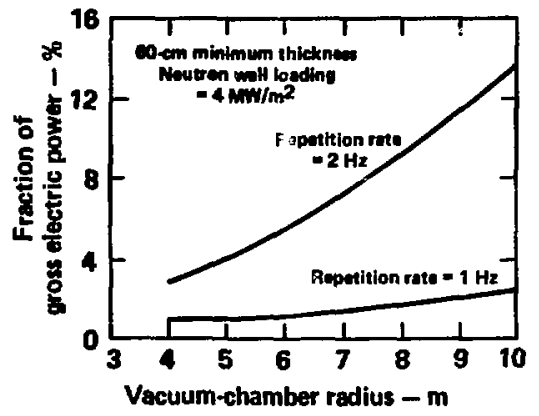

Fig. 2-25. Fraction of gross power requlred to circulate a single li, lit lithere fall.

$110 \mathrm{~m}^{3} / \mathrm{s}$, the reference reactor would require seven of these large pumps operating at $15.8 \mathrm{~m}^{3} / \mathrm{s}$ $(250,000 \mathrm{gpm})$ each.

The bithium flow rate to the heat exchangers is only a fraction of the flow rate required to recirculate and inaintain the fall. For a given temperature drop in the heat exchanger, the primary coolant flow rate is directly proportional to the rate at which energy must be removed from the reactor vessel:

$$
\dot{\mathbf{m}}=\frac{\mathrm{P}}{\mathrm{C}_{\mathrm{P}} \Delta \mathrm{T}} \mathrm{kg} / \mathrm{s},
$$

where

$$
\mathbf{P}=\text { reactor power, } \mathbf{M W}_{\mathrm{th}}
$$

$\mathrm{C}_{\mathrm{P}}=$ specific heat capacity, $\mathrm{MJ} / \mathrm{kg} \cdot{ }^{\circ} \mathrm{C}$

$\Delta \mathbf{T}=$ temperature drop at heat exchanger, ${ }^{\circ} \mathrm{C}$.

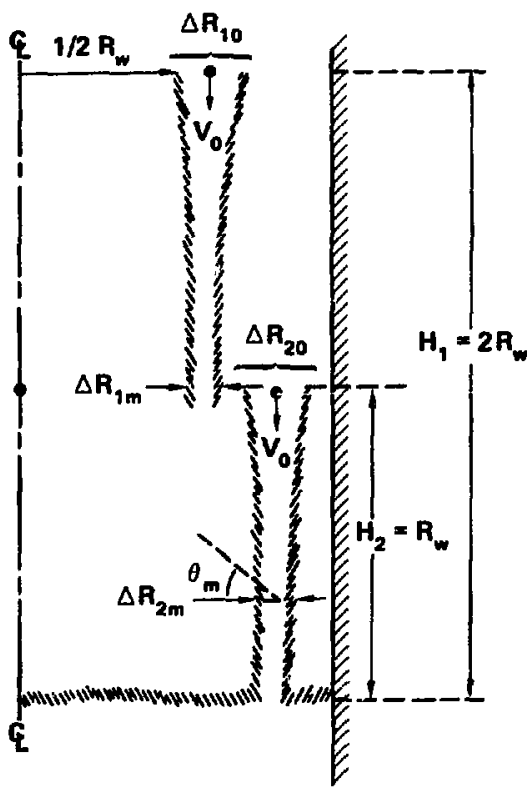

Pumping power $\simeq\left(1 / 2 \mathrm{~V}_{0}{ }^{2}+g H_{0}\right) \rho V_{0} A_{0}$ Constraints:

- Fall clearing ratio of at least unity

$$
A_{w} \leqslant V_{0} \tau+1 / 2 g \tau^{2}
$$

where $1 / \tau=$ repetition rate

- Minimum effective fall thickness of at least $60 \mathrm{~cm}$

$$
\begin{aligned}
& \Delta R_{1 m} \geqslant 60 \mathrm{~cm} \\
& \frac{\Delta R_{2 m}}{\cos \theta_{m}} \geqslant 60 \mathrm{~cm}
\end{aligned}
$$

- Total inlet thickness less than 1/2R.

$$
\Delta R_{10}+\Delta R_{20}<1 / 2 R_{w}
$$

Fig. 2-26. Recirculation pumping power calculationdouble fall. The equivgleat height is $H_{\text {, }}$, considering the multiple falls.

The required lithium flow rate to the heat exchangers is plotted in Fig. 2-29 for an assumed $\Delta T$ of $200^{\circ} \mathrm{C}$. Since the total reactor thermal power, $P$, is proportional to the neutron wall loading, $L$, it follows from the previous equation that the coolant flow rate is also proportional to L. For a system 


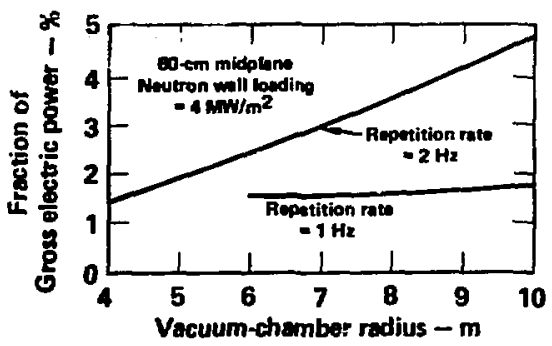

Fig, 2,27. Fradion of gross poner required to recirculate liq cis linitum fall-doeste fall.

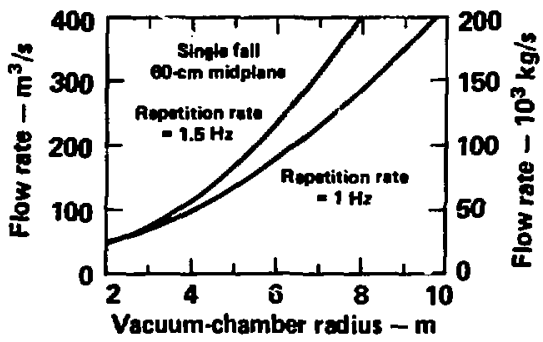

Fig. 2-28. Litwing flow rate required for the fall.

operating at other than $1 \mathrm{MW} / \mathrm{n}^{2}$, the values in Fig. $2-29$ must be multiplied by the appropriate value of $L$. Thus, our reference $4 \mathrm{~m}$-radius system operating at $4 \mathrm{MW} / \mathrm{m}^{2}(1160 \mathrm{MW}$ th $)$ requires a primary circuit flow rate of $2.8 \mathrm{M}^{3} / \mathrm{s}$; this is les. than $3 \%$ of the flow rate required to recirculate the fall.

As previously noted, a single microexplosion vauses only a small temperature change in the lithium in the vacuum chamber (assuming complete mixing). From the fall flow conditions and reactor dimensions, the arzount of lithium in the chamber at the time of the microexplosion can be determined. For most cases, it is equal to the mass flow rate divided by the pulse repetition rate. The reference reactor design at about $110 \mathrm{~m}^{3} / \mathrm{s}$ and 1.4 pulses per second contains about $\mathbf{4 0}$ metric tons of lithium at the time of the microexplosion. The 700 . MJ pulse deposits the majority of its energy in the fall (see Fig. 2-17) but only raises the temperature

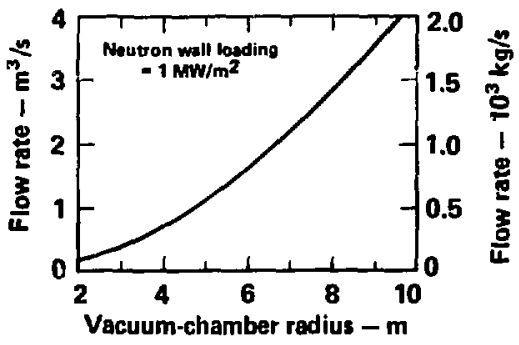

Fig. 2-29. I.lihium fow rate to heal exshanker.

Tabte 2-6. Typical pump parameters of a specific reactor design.

\begin{tabular}{|c|c|c|}
\hline & $\begin{array}{c}\text { Typical } \\
\text { pump partmeters }\end{array}$ & Unit \\
\hline $\begin{array}{l}\text { Per } \\
\text { sage }\end{array}$ & $\begin{array}{l}\text { Specific speed } \\
\text { Suction head } \\
\text { Flow rate } \\
\text { Developed head } \\
\text { Brake horsepower } \\
\text { Pump and drive } \\
\text { weight } \\
\text { Pump length } \\
\text { Pump diameter } \\
\text { Shaft speed }\end{array}$ & $\begin{aligned} \mathbf{S}_{\mathrm{N}}= & 8000 \\
\text { NPSII }= & 37 \mathrm{ft} \\
\mathbf{Q}= & 250,000 \mathrm{gpm} \\
\mathbf{H}= & 50 \mathrm{ft} \\
\mathbf{P}= & 2100 \mathrm{hp} \\
& \left(\rho=0.49 \mathrm{~g}^{\prime} \mathrm{cm}^{3}\right) \\
W= & 75 \mathrm{tons} \\
\mathbf{L}= & 8 \mathrm{ft} \\
D= & 9 \mathrm{ft} \\
\mathbf{S}= & 260 \mathrm{rpm}\end{aligned}$ \\
\hline
\end{tabular}

$4^{\circ} \mathrm{C}$. Of course the reason this is so much less than the $200^{\circ} \mathrm{C} \lambda \mathrm{T}$ at the heat exchanger is that the flow rate of the fall is about 40 times greater than the primary coolant flow rate for this particular case.

Areas of Future Investigation. This initial evaluation of the liquid lithium waterfall concept has been encouraging. The protection afforded by the thick fluid curtain of lithium will allow first wall and hlanket structures to retain their integrity for the life of the plant. In addition, constraints on vacuum conditions, tritium breeding, and energy removal are easily met. Analysis of the liquid lithium waterfall concept is continuing; some aspects currently being investigated include:

- The hydrodynamic response of the fall to $x$ ray, debris, and neutron energy deposition.

- The formation and stability of free surface annular flow.

- The compatibility of various metals with lithium and $\mathrm{Pb}-\mathrm{Li}$ alloys. 
- Methods of recovering tritium from the fluid stream.

- Neutron activation analysis.

\section{Preliminary Conceptual Design of a Liquid Lithium Waterfall Reactor}

To evaluate the performance of the liquid lithium waierfall reactor concept in the context of a power plant, a preliminary conceptual design study of a laser fusion power plant (Fig. 2-30) that incorporates this reactor soncept has been completed. The characteristics of this conceptual power plant are presented in Table 2-7. Energy processing begins with the input of electrical power from the swituhyard to the laser system. The laser system iequires $70 \mathrm{MW}$ of input electrical power to produce coherent light energy with an overall efficiency of $2 \%$. The light energy interacts with the deuteriumtritium fuel in the reactor to produce thermal energy with a gain of $Q=700$. Specifically, fusion targets producing $700 \mathrm{MJ}$ of thermonuclear energy are ignited by a $1-\mathrm{MJ}$ laser at the rate of 1.4 pulses per second. The fusion neutron energy, accounting for approximately $68 \%$ of the total yield, is multiplied by 1.24 in the lithium blanket, resulting in a power plant with a single-chamber thermal power output of $1160 \mathrm{MW}$. The thermal energy is converted to electrical energy at $38 \%$ efficiency. The laser waste heat is discharged at an average temperature of $350^{\circ} \mathrm{C}$. As a result, this waste heal can be converted to electrical energy with an overall efficiency of approximately $25 \%$. Of the total $460 \mathrm{MW}_{\mathrm{e}}$ produced, $80 \mathrm{MW}$ of clectrical power is recirculated for plant operation (laser and lithium flow). This results in a power plant with a net electrical production of 380 $M W_{\mathrm{t}}$ and an overall system efficiency of $31 \%$.

A significant feature of the plant layout shown in Fig. 2-30 is that the laser system is not housed in the reactor containment building. Several advantages result from scparating the large volume laser system from the reactor. First, building cosis and construction time are substantially reduced; second, access to the laser system for maintenance and operation is increased: finally, it becomes easier to isolate the final optics from vibrational disturbances.

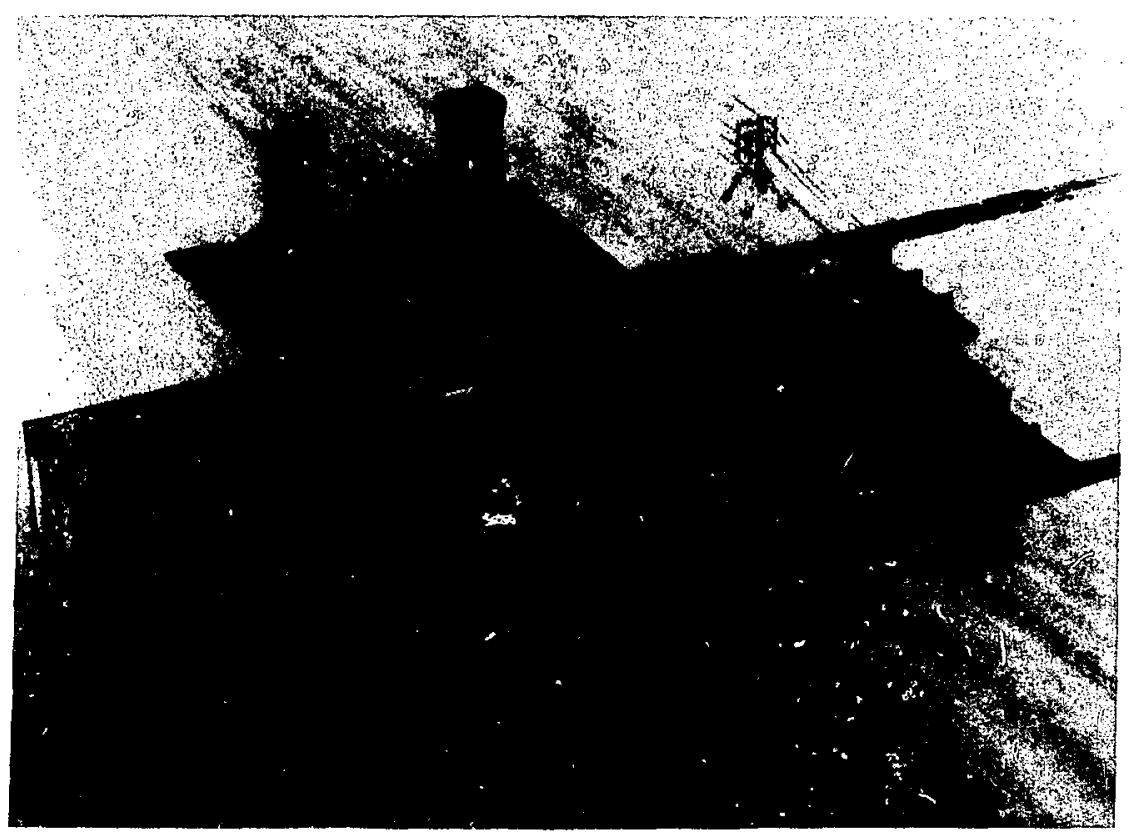

Fig. 2-30. Conceptual layout of a laser fusion power plant. 
Table 2-7. Characteristics of a conceptual power plant.

\begin{tabular}{ll}
\multicolumn{2}{c}{ Overall Performance } \\
\cline { 2 - 2 } Thermal power & \\
Fusion thermal power & $1160 \mathrm{MW}$, \\
Gross electrical power & $1000 \mathrm{MW}^{\prime}$ \\
Net electrical power & $380 \mathrm{MW}_{\mathrm{e}}$ \\
Thems efficiency & $38 \%$ \\
Recirculating power fraction & $17 \%$ \\
System efficiency & $31 \%$
\end{tabular}

\section{Laser and Pellet Parmetcrs}

\section{Total laser enery}

Pule repetition mte

Total laset power

Pellet gin (Q)

Petlet yield

Leer system efficiency $\left(\eta_{1}\right)$

Number of lase modules

Number of twobeam transport tubes

\section{Reactor Parameters}

\begin{tabular}{ll}
\multicolumn{1}{c}{ Geometry } & Cylindrical \\
\cline { 2 - 2 } Imner radius & $4 \mathrm{~m}$ \\
Height & $8 \mathrm{~m}$ \\
Lithium fall thickness & $0.6 \mathrm{~m}$ \\
Fquivalent structural wall nux ${ }^{\mathrm{a}}$ & $4.0 \mathrm{MW} / \mathrm{m}^{2}$ \\
Tritium breoding ratio & 1.7 \\
Blanket energy mulliplication & 1.24 \\
Reactor materists & \\
$\quad$ Structure & Stainless stecl \\
Cuolant & Lithium
\end{tabular}

a Flux on a dry wall at $4 \mathrm{~m}$ radius from a $14 \mathrm{MeV}$ source that inciudes $80 \%$ of the $1000 \mathrm{MW}_{\mathrm{t}}$ fusion power.
The laser light is transported to the reactor via evacuated pipes. The final focusing mirrors are located at the turning point of the beam tubes in a direct pathway to the thermonuclear microexplo. sion. As a result, these mirrors will be exposed to the $x$ rays, debris, and neutrons from the fusion reaction. Therefore, they have been placed far enough away from the microexplosion $(\sim 100 \mathrm{~m})$ to reduce the damage rates by two orders of magnitude, thus assuring their survival. Optical components such as spatial filters prevent radiation leakage from entering the laser building via the laser beam paths.

In conclusion, a preliminary conceptual design of a laser fusion power plant based on advanced highgain targets has been completed. A parametric analysis of this system was conducted to reveal attractive design points and to show the sensitivity of the design to uncertainties in both the physics and technology. Although still highly conceptual, this power plant design has provided preliminary understanding of the integrated engineering requirements and potential problems. We are encouraged by the fact that no major problems were found for which there are not reasonable conceptual solutions.

On the basis of the encouraging results of this study, a more definitive conceptual design study will be carried out during the coming year. The liquid lithium waterfall reactor will be coupled with one of the more promising laboratory stage lasers, and the major power flant components (reactor, laser, lithium system, and optical (ransport system) will be analyzed in detail. The plant layout and costing of the design will also be inciuded. Because we want to take advantage of the relevant expertise and developed technology in the nuclear fission industry, we intend to engage a reactor vendor and a power plant architect-engineer to assist us with the reactor design, plant layout, and costing portions of the conceptual design. 


\section{References}

1. J. Young et al, An Environmental Analysis of Fusion Power to Determine Related $R \& D$ Needs, Battelle Northwest Laboratories, BNWL 2010-2030 (1976).

2. T. Kabele, A. Johnson, and L. Mudge, Tritium Source Terms for Fusion Power Plants, Battelle Northwest Laboratories, BNWL 2018 (1976).

3. H. Avii and G. Kulçinski, The Effects of a Liquid ISSEC on Radiation Damage Parameters in Laser Fusion Reactor First Walls, to be published.

4. L. Sagan, "Health Costs Associated with the Mining Transport and Combustion of Coal," Nature 250, (1974).

5. An Assessment of Accident Risks in U.S. Commercial Nuclear Power Plants, United States Atomic Energy Commission, Washington, D. C., Rept. WASH 1400 (1974).

6. W. O. Allen et al., A Laser Fusion Hybrid Design Siudy, Lawrence Livermore Laboratory, Rept. UCRL-13720 (1976).

7. S. Keeny, Jr. et al., Nuclear Power Issues and Choices, Ch. 3 (Ballinger Publishing Company, Cambridge, Mass., 1977).

8. Pool-Loop-Hybrid Liquid Metal Fast Breeder Reactor Plant Comparison, Vols. 1 and 5, Rockwell International and Burns Roe Team Report FBR-76-1 (1976).

9. Prototype Large Breeder Reactor and Target Plant, Vol. 1, General Electric Co. and Bechtel Corp. Team Report NEDM-14117 (1976).

10. S. Keeny, Jr. et al., Nuclear Power Issues and Choices, Ch. 4 (Ballinger Publishing Company, Cambridge, Mass., 1977).

11. H. W. Liepmann and A. Roshko, Elements of Gasdynamics (John Wiley \& Sons, New York, 1957).

12. R. R. Buntzen and C. K. Rhodes, Laser Systems for Laser Fusion, Lawrence Livermore Laboratory, Rept. UCRL-75367 (1974).

13. J. R. Murray and C. K. Rhodes, "The Possibility of High Energy Storage Lasers Using the Auroral and Transauroral Transitions of Column VI Elements," J. Appl. Phys. 47 (1976).

14. K. Hohla and K. Kompa, "The Photochemical lodine Laser," in Handbook of Chemical Lasers, R. W. F. Gross and J. F. Bott, Eds. (Wiley-Interscience, New York, 1976), p. 667.

15. J. C. Swingle, C. E. Turner, Jr., J. R. Murray, E. V. George, and W. F. Krupke, "Photolytic Pumping of the lodine Laser by XeBr," Appl. Phys. Lett. 28, 7 (1976).
16. G. Brederlow, K. J. Witte, E. Fill, K. Hohla, and R. Volk," The Asterix III Pulsed HighPower lodine Laser," IEEE J. Quantum Electronics OE-12, 2 (1976).

17. W. F. Krupke and E. V. George, High Average Power, Rare Gas Halogen-Pumped Iodine Laser for Fusion Applications, Lawrence Liver. more Laboratory, Rept. UCRL-77523 (1976).

18. H. E. Bennett and P. C. Archibald, "Optical Requirements for Laser Mirrors," in Laser Induced Damage in Optical Materials: 1974, A. J. Glass and A. H. Girenther, Eds. (National Bureau of Standards, Washington, D. C., Special Publication 414, December 1974).

19. J. Hovingh, "First Wall Studies of a LaserFusion Hybrid Reactor Design," Proc. 2nd Topical Meeting on the Technology of Controlled Nuclear Fusion, Vol. II, 765, Richland, Washington (1976).

20. J. Hovingh, J. A. Maniscalco, M. Peterson, and R. W. Werner, "The Preliminary Design of a Suppressed Ablation Laser Induced Fusion Reactor," Proc. Ist Topical Meeting on the Technology of Controlled Nuclear Fusion, Vol. I. 96, San Diego, California (1974).

21. L. A. Booth (compiler), Central Station Power Generation by Laser-Driven Fusion, Los Alamos Scientific Laboratory, Rept. LA-4858MS, Vol. I (1972).

22. D. A. Freiwald, T. G. Frank, E. A. Kern, and L. A. Booth, Laser-Fusion Generating Stations Based on the Magnetically Protected Reactor Cavity, Los Alamos Scientific Laboratory, Rept. LA-UR-75-2035 (1975).

23. M. Sparks, Xonics Corporation, Santa Monica, California, private communication (1977).

24. E. F. Plechaty and J. R. Kimiinger, TARTNP: A Coupled Neutron-Photon Monte Carlo Transport Code, Lawrence Livermore Laboratory, Rept. UCRL-50400 Vol. 14 (1976).

25. R, J. Howerton et al., An Integrated Sysiem for Production of Neutronics and Photonics Calculuted Constants, Vol. I5, The LLL Evaluated Nuclear Data Library (ENDL) Lawrence Livermore Laboratory, Rept. UCRL-50400 (1975).

26. A. P. Fraas, Comparative Study of the More Promising Combinations of Blanket Materials. Power Conversion Systems, and Tritium Recovery and Containmens Systems for Fusion Reactors, Oak Ridge National Lab, Rept. ORNL-TN-4999 (1975). 
27. G, Davey, "Cryopumping in Transition and Continuum Pressure Regions" Vacuum 26, 17. 22 (1976).

28. V. L. Streeter, Fluid Mechanics (McGraw-Hill,
Inc., New York, 1966) pp. 301-306.

29. B. Atz, Liquid Metal Engineering Center, Canoga Park. California, private communication (April 1977). 


\section{A CONCEPTUAL DESIGN STUDY FOR A LASER-FUSION HYBRID}

Neutronic results assessing the potential of a laser-driven fusion-fission hybrid reactor have been previously published $\mathbf{1 . 2}$ and are presented in the executive summary. However, more accuratc assessments are needed, us well as a definitivc ranking of the more promising concepts. This requires studies dealing with enginecring, safety, and economic issues, as well as neutronic results. With this in mind, we undertook a conceptual design study of a laser-fusion hybrid in cooperation with Bechtel Cotporation. The study was to provide enough design detail to realisticully gauge the value of a laser-fusion hybrid in a power-gencrution cconomy based on fission reactors. The joint effort began in July 1975. This chapter describes and analyzes the laser-fusion hybrid design which has evolved.

The hybrid concept chosen for this study is a depleted-uranium-fucled, fast-fission blanket that produces fissile plutonium and electricity. It emphasizes fissile-material generation by maximizing fuel production at the expense of energy production. Selection of this blanket material was based on reported neutronic results ${ }^{1,2}$ that indicated it could provide enough fissile fuel to extend $L W R$ energy production as much as two orders of magnitude.

$A$ comparative analysis ${ }^{1}$ of the neutronic properties of several hybrid concepts has shown that depleted-uranium, fast-fission blankets produce large amounts of fissile fuel (per unit of thermal energy) with low laser-efficiency and larget-gain requirements. The depleted-uranium blanket selected for our conceptual design produces enough fissile matcrial to continuously fucl more than six LWRs of equivalent thermal power. Thorium-fueled hybrids produce more fissile fuel per unit of thermal energy, but have a lower energy-multiplying capability and require a higher performanee (more costly) laser-fusion system. Blanket concepts exist with higher energy-multiplication capabilities than depleted-uranium blankets, but they enhanee energy multiplication at the expense of fissile production.

Light-water reactors will be the major, and most likely the only, source of commercial, nucluar elecIric power this century. Their growth will strongly depend on the supply of fissile fuel. By converting the ${ }^{238} \mathrm{U}$ in natural uranium to fissile plutonium, the hybrid could extend the fissile-fuel supply for the economical LWRs. Fast breeder reactors also offer the prospect of more fully utilizing the uranium resources, but they will not provide fissil? fuel for LWRs. Excess material from a fast breeder reactor is best used to fuel another fast breeder reactor. Therefore, the growth of fast breeder reactors will depend entirely on their economic competiveness with other power plants.

\section{The Laser-Fusion-Hybrid Power-Plant Design}

In the joint laser-fusion, hybrid-design study, l.l. provided the overall direction, the neutronics datit, and the fusion portions of the design. Bechtel Corporation provided the fission portion of the hybrid, the design of the thermal-energy transport and conversion system, the tritium-recovery system, and the layout of the complete power plant. They also analyzed the fuel cycle, the capital involved, and the operating costs. Bechtel's contribution to the laser-fusion hybrid design is fully documented in their final report. ${ }^{3}$

The four major objectives in the design study were:

- Identify the laser and pellet performance levels required to economically produce fissile fuel and power with a hybrid.

- Evaluate the integrated engineering performance of a complete conceptual design.

- Compare a laser-fusion hybrid to existing fission-breeder options (LMFBR, GCHBR, LWBR).

- Identify major technological problems associated with a laser-fusion hybrid.

These objectives defined the level of design detail and costing analysis for the study.

In addition, a few philosophical points of view significuntly affected our design choices. First, we wanted to operate the laser-fusion hybrid in a regime that, compared to pure fusion, required an order-of-magnitude lower laser/pellet performance, i.e., fusion energy gains in the neighborhood of 1.0 . This meant the blanket energy multiplication had to be about 10.0. Second, we wanted to use state-ofthe-al't fission technology in the design of the hybrid blanket. In keeping with this principle, we chose stainless steel as the structural and cladding material instead of higher performing refractory metals. Finally, we believed that a hybrid reactor which produces fissile fuel should be designed with the same safety standards and environmental impact as the fissile burning reactors for which it is providing fuel. Only a negligible improvement in 
the overall environmental impact results fron making the hybrid environmentally more attractive than the more numerous LWR's.

\section{Hybrid Reactor Design}

The functional shape of the laser-fusion hybrid chosen for final evaluation is shown in Fig. 3-1. In its simplest form it is a cylinder with a height-todiameter ratio of 1.0. The center of the fusion chamber is the focal point for a six-beum, 100-kJ laser system which irradiates the fusion target from the top and bottom of the cylinder.

The basic features of the hybrid reactor are displayed in Fig. 3-2. A depleted-uranium-fueled, fastfission blanket radially surrounds the fusion chamber. The energy in the fission zone (amounting to $90 \%$ of the tolul energy) is rumoved with a sodium coolant system. The liqujd sodium flows from the lower plenum to the upper plenum through hexagonal process tubes.

Lithium-cooled, graphite-moderated blankets are positioned in the top and bottom of the reactor and behind the fission zone. These lithium blankets capture neutrons and breed tritium. The laser beams and pellet injectors penetrate the top and bottom blankets leaving the radial fission blanket unencumbered. We had originally intended to also use fission blankets in the top and bottom regions, but maintaining coolant flow while removing the top blanket for access to the fusion chamber would have been difficult. In addition, this would have required an unconventional lission blanket design, which was not in keeping with our desire to use state-of-the-art technology. Our decision resulted in a $30 \%$ reduction in both fissile fuel and energy production.

As shown in Fig. 3-3, the entire blanket system is enclosed in a spherical stainless-steel vacuum vessel with a removable top. The final focusing mirrors are placed in beam tubes outside the vacuum vessel to minimize damage caused by the fusion microexplosion and to allow replacement.

\section{Fission-Blanket Design}

Af expanded side view and top view of the radial fission blanket are shown in Figs. 3-4 and 3-5. The fission zone consists of two rows of hexagonal process tubes that contain the depleted uranium as stainless-steel-clad fucl pins. The process tubes in the inner row are protected from the fusion microexplosion by a stainless-stecl-supported graphite liner.

Our neutronic calculations indicate that energy multiplication and fissile-fuel production are maximized by using uranium-metal as fuel instead of oxides or carbides and by maximizing the ratio of

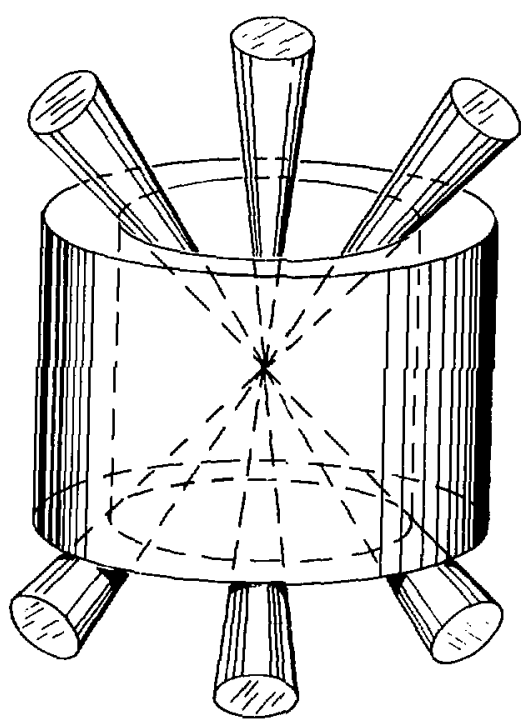

Fig. 3-1. The geometry used in the 1.I,t./lkehtel Iaser-fusion hybrid.

uranium volume to structural material. Increasing the residence time of the fuel also increases the average energy multiplication since more energy is produced ats plutonium accumulates atd fissions in the blanket.

Fast-ncutron damage may limit the useful life of stainless steel and other structural inetals in a fusion reactor. A common design criteria for both laser and magnetic-fusion reactors is a first-wall neutronflux Jimitation of $1 \mathrm{MW} / \mathrm{m}^{2}$ and an expected lifetince of less than five full-power years. Neutronic calculations at a first-wall loading of $1 \mathrm{MW} / \mathrm{m}^{2}$ show a maximum power density in a depleteduranium blankel of about $8 \mathrm{~W} / \mathrm{g}$ or about 150 $W / \mathrm{cm}^{3}$ of uranium metal. The average power density in a blanket is less than $4 \mathrm{~W} / \mathrm{g}$. Five full-power years of blanket operation would result in an average burnup of less than 7,000 MWD/tonne of uranium. This low rate of burnup favors the choicc of metallic fuel.

The low power density and burnup capabilities of a depleted-uranium (or a natural-uranium) blanket demand more care in minimizing fuel-cycle costs, including fabrication cost. Thus, large fuel rods are favored over small rods, and long fuel elements have some advantage over short elements. 


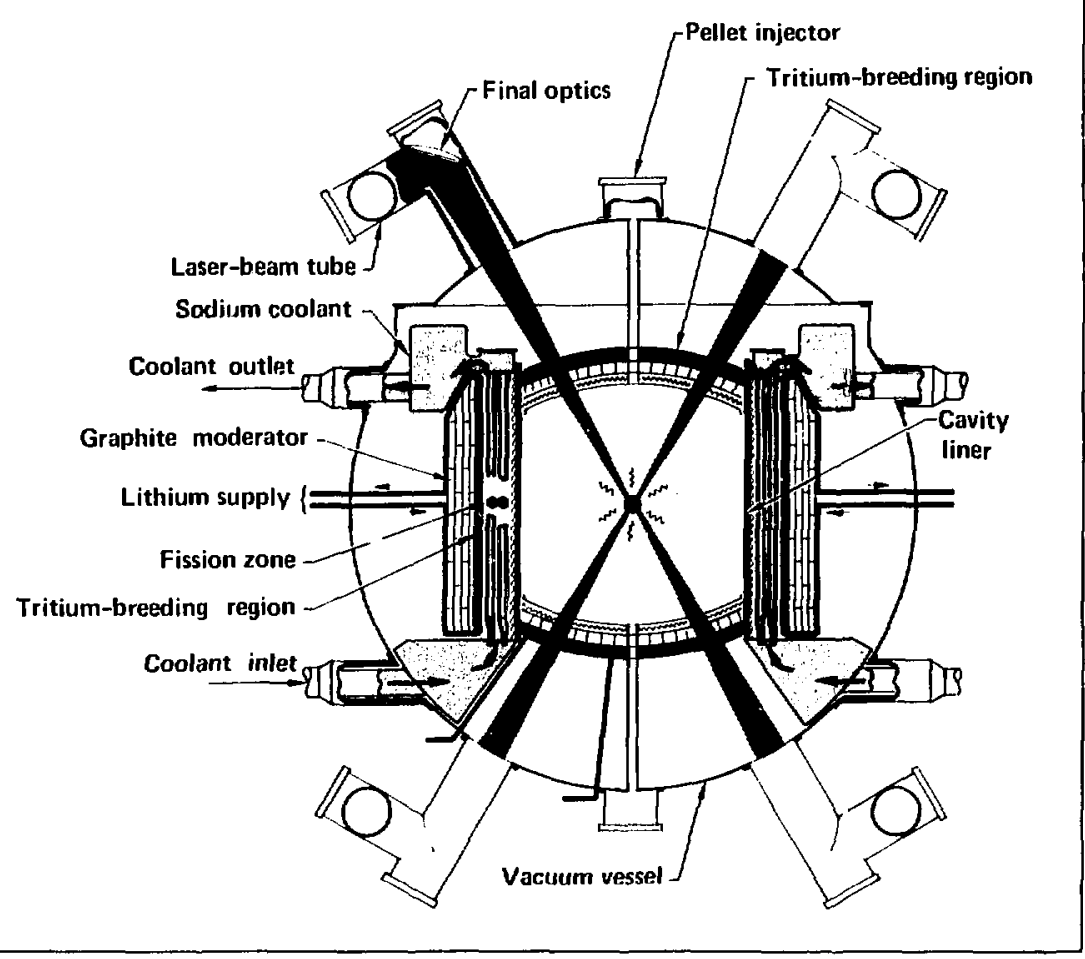

Fig. 3-2. Side view of u cunceptual laser-fosion hyhrid.

The basic configuration of a latser-fusion reactor-a vacuum chamber with laser beams converging from severil angles-introduces difficulties in the mochanical design of the hlanket. If a thick pressure-vessel wall is introduced between the blanket and the fusion core, neutronic performance is severely reduced. The fuel element must therefore operate and be cooled within a surrounding vacuum. Thin process tubes and low-pressure coolants seem to be the most reasonable design approach. but coolant leaks and process-tube reliability will always be potential problems. We expect that the melting of the fuel after loss of coolant will be the most serious safety issue with the hybrid.

\section{Selection of a Fuel Material}

Our goal wats to design a system that would maximize neutron energy multiplication, minimize fuelcycle costs, and be licensed by the Nuclear Regulatory (ommission. This goal led to selection of uranium-metal fuel elements with sodium as the coolant. L.MI:BR technology and balance-of-plant design concepts were used as much as possible. The selected fucl element is a 19-rod cluster similar to those developed during the carly 1960s for use in sodium-graphite reactors (SGR), lue] rods 18 feet ling were developed at that time. However, because of their flexibility and tendency to buckle if not kept 

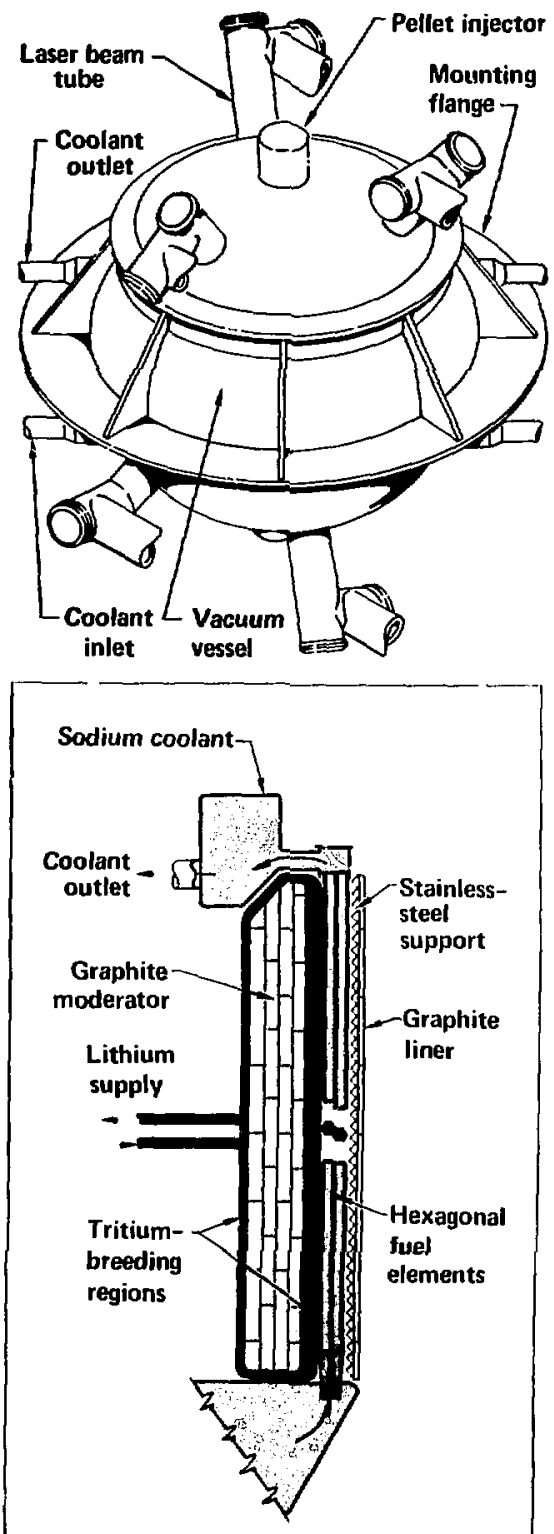

Fig. 3-4. Side view of assion blanket in the fascerfusion hybrid reactor.
Fig. 3-3. Vacuum resset for the laser-funisn hybrid zeactor.

vertical, they required extra care in handling. Adapting this experisnce to a blanket design with harger diameter fuel rods and a thicker cladding, we decided to use 7-no (23-ft)-long fucl rods and assemblics: this determined the height of the fusionreactor core. $A$ three dimensional view of a fuel ussembly is shown in Fig. 3-6.

Uranium metal, uranium with $7-\mathrm{w} / \%$ molybdenum alloy (U-7 Mo), and uranium carbide (UC) were all considered as fuel malcrials for the sodiumcooled blankel, and all are satisfactory. The fucl elcment chosen can aceept 30-mm-diameter fuel slugs of each of these matcrials inlerebangeably. The U-7 Mo fuel should he capable of burnups to $20,0 \times 0$ MWI)/tonne of uranium at maximum center temperatures of $650^{\circ} \mathrm{C}$. This burnup would require al leasl eight full-power years, a period the cladding probably could not tolerate. The U.7 Mo alloy was the reference fuel for the Dounreay list reacedor and performed antislactorily at the conditions noited. This alloy has energy inultiplication and plutoniumproduction rates that are reduced, compared to those of uraniute metal; it is also more expensive to fabricitle and reprocess. Uranium earbide fuel would be capable of more latan 100.000

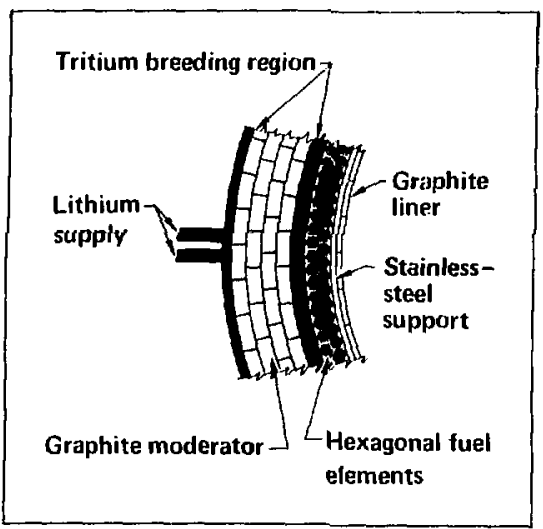

Fig. 3-6. Top view of fission blanket in the laser. fusion hybrid reactor. 


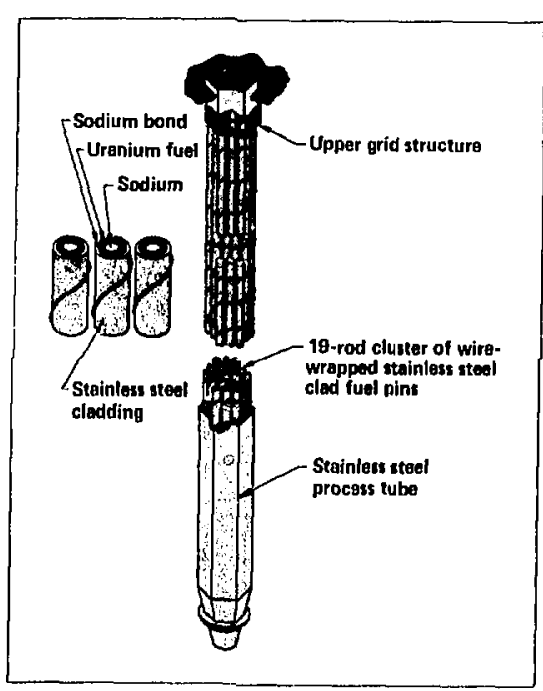

Fig. 3-6. Fuel elenient and process tule configuration.

MWD/tonne of uranium burnup at maximum temperatures of $1,000^{\circ} \mathrm{C}$ (with a sodium bond), if the cladding were adequately strong. However, its energy multiplication is $30 \%$ less than uranium metal. In the low-power-density configuration of the chosen blanket, the higher burnup capabilitics of U.7 Mo and UC cannot be used effectively. If higher power densities werc possible (by using firstwall fuxes of 3 to $4 \mathrm{MW} / \mathrm{m}^{2}$ or fissile-enriched tranium fuel), or if gas cooling were used, UC would probably have been chosen.

Uranium metal, adjusted with minor alloying additions to control swelling, was chosen as the fuel niaterial for several reasons; its multiplication and breeding performance were superior, and its burnup capability was judged to be adequate and a good fit to the blanket's low-power density. Finally, it was cheaper to fabricate and reprocess.

Pure uranium metal begins to swell disasterously at temperatures gretater than $400^{\circ} \mathrm{C}$ and burnups greater than 1,000 MWD/tonne of uranium (about 0.1 atom percent fissions). The British development of adjusted uranium metal as a fuet for their gascooled Magnox reactors during the early 1960 s demonstrated that additions of 800 to $1,000 \mathrm{ppm}$ Al, 350 to $500 \mathrm{ppm}$ Fe, and approximately $500 \mathrm{ppm}$ carbon could control this swelling and allow burnups of 5,000 to $6,500 \mathrm{MWD} /$ tonne of uranium at temperatures of $600^{\circ} \mathrm{C}$. Extensive development of similar alloys using $\mathrm{Al}, \mathrm{Fe}$, and $\mathrm{Si}$ (and sometimes Mo) have taken place at the Savannah River Laboratory and at Battelle Northwest Laboratory confirming the sitisfactory performance of these fuels. A maximum fuel temperature of $600^{\circ} \mathrm{C}$ and a maximum burnup of $6,000 \mathrm{MWD} /$ tonne of uranium were chosen as a desiga basis for the blanket. A volume increase (swelling) of $4 \%$ is expected at this burnup. However, a volume increase of $81010 \%$ is casily tolerated by the sodium-bonded fuel rod. A maximum burnup of 6,000 to 6,500 $M W D /$ tonne of uranium is probubiy a reasonable expectation for this fucl. Sodium bonding of the cladding to the fuel rod was chosen over contact bonding to accommodate fuel swelling without straining the cladding; with sodium bonding, a thinner cladding could be used.

\section{Lithium-Cooled Kadial Blanket}

The lithium-cooled radial blankel located behind the fission blanket extends from the top to the bottom of the sodiun plenums. The blanket consists of a cylindrical stainless-steet container $62 \mathrm{~cm}$ wide and $750 \mathrm{~cm}$ high. On the inside of the blanket there is first a 2-cm-thick stainless-steel inner wall, thei, 6 $\mathrm{cm}$ of lithium, $50 \mathrm{~cm}$ of stainless-stecl-clad graphite. $2 \mathrm{~cm}$ of lithium, and finally, a $2-\mathrm{cm}$-thick stainlesssteel outer wall. The lithium, which is enriched to $50 \% 1 \mathrm{li}^{6}$, enters the reactor from an inlet header and is fed into a plenum at the bottom of the radial blanket. Lithium flows upward through the two channels providerd on either side of the clad graphite and into the top pienum.

\section{Top and Bottom Blankets}

The top and bottom blankels are cylindrical, curved pancake-shaped plenums. The shell is $2 \mathrm{~cm}$ thick, and from the inside, ach blanket is constructed with $2 \mathrm{~cm}$ of stainless-steel, $10 \mathrm{~cm}$ of beryllium, $70 \mathrm{~cm}$ of graphite, $10 \mathrm{~cm}$ of lithium, and the 2-cm stainless-stecl outer shell. The blankets are located $350 \mathrm{~cm}$ from the center of the reactor. The use of beryllium in these blankets provides a tritium-breeding ratio of $\mathbf{1 . 5}$. This allows the radial blanket to operatc with a tritium-breeding ratio of 0.95 and still maintain an overall tritium breeding ratio of 1.l. Reducing the tritium-breeding requirements in the radial blanket makes it possible to use a thicker fission zone, thereby increasing the fissile fuel-production capability of the reactor.

\section{Final Laser Optical System}

Six horizontal laser beams enter the reactor through a double-mirror system located outside the 
reactor. The laser light is focused on the fusion target by six metal mirrors, three each at the top and bottom of the reactor; radiation shields are provided for each beam. The focal length of the mirrors is $12 \mathrm{~m}$, and the final mirror is designed to accommodate a beam diameter of $1.2 \mathrm{~m}$ and a maxmum energy flux of $i .5 \mathrm{~J} / \mathrm{cm}^{2}$.

To be good reflecting and focusing elements, bitrors must be smooth to approximately onefourth of the wavelength of the luser light impinging on them. Highly polished metal surfaces are susceptible to all types of radiation damage, and we are currently investigating the effects of the neutrons, $x$ rays, and energetic debris from the fusion microexplosion. The damage caused by this loading has been lessened somewhat by placing the mirror at a point where the radiation fluxes emanating from the fusion targel are more than an order of magnitude lower than the first-wall fluxes. An additional problem uncovered by our preliminary optical studies involves damage to the mirrors from the laser light as debris from the fusion chamber accumulates on the mirror surface. $A$ mechanism for removing the debris between shots must be devised.

Removing and replacing mirrors will be expensive because of the handling required. Replacing the mirrors at the bottom of the reactor will be difficult without special equipment. The lifetime of the mirrors will determine whether it is necessary to use special designs such as rotating inirrors, gals windows, magnetic-field directors, and special remole handling for replacing mirrors. We have wot considered optical-system design here, except for layout and basic laser-system functional purposes surrounding the reactor.

\section{Pellet Apparatus}

The pellets of DT must be injected into the realtor at a nominal 20 times a second and must reach an exact location without error. The apparatus must be insulated from the reactor (if frozen pellets are used) and must remain correctly aligned with the laser beams during the expansion of the reactor eaused by internal heat load. The apparatus is also subject to thermonuclear blast and the nominal reactor vacuum of 0.1 Torr. No apparatus is available today that fulfills these requirements.

\section{First-Wall Considerations}

The technological and economic feasibility of the laser-fusion hybrid are critically dependent on the design and performance of the first wall because its radius and lifetime determine both the size of the reactor for a given output power and the availability of the power plant. We have calculated the radiation-exposure capabilities of several first-wall materials and have chosen a 2-cm-thick graphite liner supported by stainless-steel and cooled with lithium. The graphite first wall is designed for an operational lifetime of one year with a neutren wall loading of $1 \mathrm{MW} / \mathrm{m}^{2}$ and a repetition rate of $20 \mathrm{~Hz}$. The nominal charged-particle loading is then 0.25 $\mathrm{MW} / \mathrm{m}^{2}$ or $12.5 \mathrm{~kJ} / \mathrm{m}^{2}$ per pulse. The chargedparticle energy is carried in the form of pellet debris and alpha particles that escape the pelles.

We have calculated the temperature rises, the stresses, and the amount of material vaporized by the interaction of $x$ rays, charged particles, and reflected light with the first-wall material. The analytical methods used for these estimations are presented in Refs. 4 and 5. The rate and depth of energy deposition in the first wall from the thermonucle:ir-burn products and the reflected laser light is dependent on several parameters including:

- Jaser wavelength.

- Jaser energy and power.

- Thermonuclear yield.

- Pellet mass and composition.

- Cas pressure in the fusion cavity.

- First-wall composition and configuration.

A computer code called I.ASNEX has been developed at l.I.l. to explore this complex parameter space." I.ASNEX is a Lagrangian hydrodynamic code that incorporales the principal physjeal processes octurring in laser-produced plasmas; it also computes the time eveiution of the basic physical characteristics of the plasma. By using I.ASN!I:X, it is possible to calculate the transport and interaction of laser photons, clectrons, jons, $x$ rays, and fusion-reaction products, as well as the induced magnetic and electric fields and the hydrodynamic behavior of the pellet.

We selected a laser target that LASNLX predicts will yield $10 \mathrm{MJ}$ of thermonuclear energy from an implosion caused by a few hundred kilojoules of 1 . $\mu \mathrm{m}$ laser light. One pereent of the fusion energy is relecised in the form of $x$ rays, $23 \%$ in charged part 1 cles, and 76\% in 14-MeV neutrons. The energy spectra and pulse widths occurring at the first wall have been determined by continuing the LASNEX calculation long enough for the $x$ rays and charged particles to interact with the cavity gas at $0.1 \mathrm{~T}$ orr. The resulting spectra and pulse widths were then input into the first-wall calculations. The resul s indicated that thermal ablation of a graphite $c$ stain $3.5 \mathrm{~m}$ from the microexplosion will resuit in a surface-recession of less than $1 \mathrm{~cm} / \mathrm{yl}$. Spallation of the graphite by temperature-induced stre'ses has also been considered in these first-wall cali slations. 
Another factor limiting first-wall lifetime will be erosion by the formation of hydrocarbons. For a D'T target with $5 \%$ burn, the graphite will erode at a rite less than $1 \mathrm{~cm} / \mathrm{yr}$, assuming that alf the hydrogen reacts with the curtain to form atetylene.

The graphite curtain must be flexible enough to witistand the thermal stress caused by reflected light and $x$-ray loadings, as well as charged-particle loading. $A$ weave of graphite fibers has hesen proposed for this purpose, but the transmission of heat through such a eloth is uncertain. It may be preferahle to use a onc-layer, Iwo-dimensional weave that is continuously replaceable as it crodes.

In sunmary, the first wall is assumed to he a 2 em-thick graphite curtuin supported on a stainlesssteel backing. The design of a cooling system for this structure is not included, and the structural design for sufficient flexibility has not been considered in detail.

\section{Analysis of the Design}

\section{System Performance}

The overall performance and the more significant design parameters of the laser-fusion hybrid are summarized in Table 3-1. A thermal output of

Table 3-1. Laser fusion hybrid dexign paranuelers.

Syatem porformante

\begin{tabular}{|c|c|}
\hline \multicolumn{2}{|l|}{ Thurmal power, $M W_{1}$} \\
\hline Puston thermal power, MW, & 200 \\
\hline Grosu electrical nower, $M W_{e}$ & 5.35 \\
\hline Net electrical power, MW & 400 \\
\hline Recirculating power fraction & 0.25 \\
\hline Syatem efficiency, $\%$ & 0.29 \\
\hline Average blanket energy multipliketion & 8.7 \\
\hline Net plutonium production, $\mathrm{kg} / \mathrm{yr}$ & 1300 \\
\hline Tots tritiun production, $\mathrm{kg} / \mathrm{yr}$ & H.0 \\
\hline Lawer energy, (KJ) & 100 \\
\hline Laver nystem efflciency, $\%$ & 2.0 \\
\hline Power supply energy, MJ & 5.0 \\
\hline Pulso repetition rate, $n^{-1}$ & 20 \\
\hline Pellet galn, Q & 100 \\
\hline Fualon enorgy gain & 2.0 \\
\hline
\end{tabular}

Optical transport system

Number of bewms

Maximum energy nux, $\mathrm{J} / \mathrm{cm}^{2}$

6

Beam dlameter, $m$

Focal length of final mirrora, $m$
$1400 \mathrm{MW}$ was chosen to emphasize that a laserfusion-driven hybrid could operate as a relatively small power unit. The fusion targets are irradiated by a 6-beam, $100 \mathrm{~kJ}, 20-\mathrm{Hz}$ laser with an overall efficiency of $2 \%$. The fusion-energy gain (i.e., the product of laser-system efficiency and pellet gain) for this retactor is 2.0 . This results in a plant recirculating power fraction of $25 \%$, and a net system of ficiency of $29 \%$. If the fusion-energy gain were inereased to 4.0, the recirculating power would decrease to $16 \%$ and the net system efficiency would increase to $32 \%$.

The performance and the design parameters presented in Table 3-I ean be placed in perspective by comparing the laser-fusion lyybrid to a typiend fast breder reactor. This comparison is shown in Table 3-2 where both systenss have been normaliaed (1) a thermal output power of $25(0) \mathrm{MW}$. The fast breeder renctor used in this exmparison is an I.Ml:3R with a breeding ratlo of 1.2. As shown, the laser-fusion hybrid generales $30 \%$ less electricial power because its driving lister requires $19 \%$ of the gross power. This inferior perfornanee in power genteration results from design choices that were influeneed by our desire to emphasisc fissile-fuel produclion at the expense of energy multiplication. The advantages of the laser-fusiont hybrid over the I.MFBR are readily apparent from Iable 3-2. Specilically, lloc hybrid produces 10 (imes more fissile misteriat, requires no initial fissile-fucl loading, and operates at one-tenth the power density. Betasuse there is no intitial fissile inventory, it is pessible to operate the hybrid in a regime where bolh criticality aceidents and core disruptive atcidents are impossible. Moreover, control rids are nol required. The luwer power densities make it possible to desigfi a liybrid bianket that provides much more tince to recover from a loss-of-coolant

Table 3-2. Comparison of laser-fusion-hybrid and fission-breceder perfornance.

\begin{tabular}{|c|c|c|}
\hline & Hybrid & $\begin{array}{l}\text { liusion } \\
\text { brucuder }\end{array}$ \\
\hline Thermal power, MW, & 2500 & 2500 \\
\hline Nex tectrital power, MW. & 725 & 1000 \\
\hline System effreiency & 0.29 & 0.40 \\
\hline Net fisaile production, $\mathrm{kg} / \mathrm{yr}$ & 2300 & 260 \\
\hline Jisstle fuel londing & 0.0 & 2500 \\
\hline $\begin{array}{l}\text { Maximum power density in } \\
\text { fuel, } w / \mathrm{cm}^{3}\end{array}$ & 150 & 1500 \\
\hline Averege power density, $W / \mathrm{cm}^{3}$ & 30 & $\sim 300$ \\
\hline
\end{tabular}

The rission brecder used in this comparison is an LMliBl? with a breeding ratio of $\mathbf{1 . 2}$. 
accident. In fact, it is technologically fuasible, and perhaps coonomically feasible, to design a hybrid blanket that passively copes with a loss-of-conlant accident.

\section{Fconomic Anglysis}

Bechtel's prelininary cost analysis of the 1400 $M W_{t}$ laser-fusion hybrid design reveisled that some of the design choices had severe economic penalties. A survey of the high-eost items indictited that the reactor-containnent strueture and several of the other buildings were much too large for the nominal output power of $4(0) \mathrm{MW}_{\mathrm{L}}$ In addition, there were severaf other bitance-of-plant items whose cosis were relatively independent of output power, thereby implying that a larger plant output power would be more ceonomicul. These results agree with scaling fictors fior other nucluar-power reactors that show an electrical-power plant is more economical in the $1200-M W_{c}$ range atnd, where possible, in twin utrits.

These results led us to perform a more detililed cost analysis on a hybrid with a larger output puwer. We sealed our conceplual design to a gross power of $1300 \mathrm{MW_{ \textrm {t } }}$ and a nel power of $950 \mathrm{M} \mathrm{W}_{\mathrm{e}}$. This out put was obtained from the original design by intercising the fatster entergy from $100 \mathrm{t}$ to $200 \mathrm{~kJ}$, increasing lowe average pulse repetition rate from 20 to $2511 \%$, and increasing the inner radius of the blanket from 3.5 to $4.9 \mathrm{~m}$.

\section{C'apital and Operating C'osils}

The cipital cost of the laser-fusion hybrid reatetor plant has been estimated from conceptual design and engineering informations. $A$ large portion of the power plant consists of eonventionil technology. such as thermil energy transfer, electrical generation, and cooling and auxiliary systens; theretore, cost estimates can be based on background experiente. Because the fusion retetor, the laserinterface, and the fusion fuel cycle are conceptual, their costs have been estimated on a fisst-of-il-kind cost basis. The operating costs of the laser-liusion hybrid reactor arc bisced upon ist nackear fust-cycke and equipment-replacernent costs in addition 10 capital charge rates and general operating and maintenance costs similar to those of $L$ M 1 HBR reactors.

Capital Costs. The results of the cost indysis are summarized in Table 3-3. The costs of the laserfusion hybrid are compared to cost estimates for a typieal L.WR. All of the cost estimales have been inade at first quarter, 1976, price and wage levels and no allowance has been made for future escalation. The Nuclear Steam Supply System (NSSS) calcgory for the hybrid includes the reactor vessel with its internals, and the primary-coolant loop with its associated pumps, motors, heat exchangers, and steam generators. Major items included in the "other mechanical" category are the turbine generators, the vacuum system, the tritium system, and the cooling towers. Site improvements, the r:actor-containment structure, and all other buildings make up the "civil and structural" calcgory.

The indirect costs in Table 3-3 were estimated on the basis of a tine-year construction of the LWR ind a 10.5-yeitr construction of the more complex laser-fusion hytrid. Thas, the indirect costs for the hybrid account for a larger fraction of the total eapital cosit. licld costs are the items in the construction cost which cannot be direttly ascribed to the capital costs. They include temporary constcuction facilities, supply and maintenance of construction equipment and tools, field-office operation,

Table 3-3. Capifal and uperating cost andysis.

\begin{tabular}{|c|c|c|}
\hline Capilal unt item (MS) & $\begin{array}{c}\text { I.WR, } \\
1200 \mathrm{mw}_{\mathrm{e}}\end{array}$ & $\begin{array}{l}\text { Imer-fusion } \\
\text { Jubrid, } \\
950 \mathrm{MW}\end{array}$ \\
\hline $\begin{array}{l}\text { Nuclear Steam Supply } \\
\text { Syutem (NSSS) }\end{array}$ & 78 & 268 \\
\hline Other Meclıanical & I01 & 201 \\
\hline C'ivil and Struclural & 142 & 158 \\
\hline Pipiug & 77 & 105 \\
\hline Instrumentation & 9 & 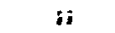 \\
\hline Iftectrical & 43 & 72 \\
\hline Total direct costs & 450 & B15 \\
\hline J'juld cusals & 79 & 171 \\
\hline linginecring survices & 80 & 197 \\
\hline Contingetuly & 91 & 272 \\
\hline Owners tost at $7 \%$ & 56 & 116 \\
\hline $\begin{array}{l}\text { Interest during construction } \\
\text { at } \mathrm{B} / \mathrm{m}\end{array}$ & $\begin{array}{r}197 \\
(9 y x)\end{array}$ & $\begin{array}{r}487 \\
(10.5 \mathrm{yr}) \\
\end{array}$ \\
\hline Total indirect costh & 503 & 1243 \\
\hline Total Cost & 953 & 2058 \\
\hline Cost por kw installed, s & 794 & 2166 \\
\hline \multicolumn{3}{|l|}{ Operating Cost Itcm, mills/kWh } \\
\hline Copite" & 19.42 & 55.77 \\
\hline fiucl & 6.3 & $(-3.17)$ \\
\hline Operating and mpintenance & I.S & 2.40 \\
\hline Total operating costs & 27.22 & 55.00 \\
\hline
\end{tabular}


and acceptance testing. The engineering services include all enginecring and home-office costs and fres. Included in the indirect cost is a contingency allowanc: for the uncertainty that exists within the conceptual design in quantity, pricing, or productivity.

The total capital cost of the laser-fusion hybrid is estimated to be $\$ 2,058$ million. Thus, on a cost-perkjlowatt installed basis, the hybrid is 2.7 times more expensive than the LWR. It should be noted that this cost estimate does not include the laser system or the pellet-manufacturing facility. If $\$ 200$ million are allowed for these omitted facilities the laserfusion hybrid would cost approximately three times more than a typical $\mathbf{L W R}$.

Operating Cost. The cost of electricity from the hybrid is 55 mills/kWh. This is approximately twice as much as the cost of electricity from the LWR. The capital portion of the operating cost is by far the dominant factor in the cost of electricity. It has been estimated for both reactors on the basis of a $15 \%$ rate of return on the capital invested. The fuelcycle cost for the laser-fusion hybrid is negative because revenues are oblained from the sale of the plutonium it produces. The cost bases used to estimate the fuel cycle cost for both the LWR and the hybrid are presented in Table 3-4. The fabrication cost for the hybrid is cheaper because the cladding material is stainless-steel and the cross-sectional area of the fuel pin is much larger. The spent-fuel shipping and reprocessing costs are less for the hybrid because it operates at lower average burnups ( 6,000 vs $33,000 \mathrm{MWD} /$ tonne of uranium).

The major issue concerning a laser-fusion hybrid is not how much it will cost, nor the price at which it can generate electricity, but rather the cost of electricity when hybrids are providing fissile fuel for existing burner reactors. In Fig. 3-7, the cost of electricity has been plotted as a function of the cost of fissile fuel for hybrids with varying capital costs. These results indicate that the cost of electricity is quite insensitive to the capital cost of the laserfusion hybrid. Specifically, the cost of electricity increases by only 20 to $40 \%$ when the capital cost of the hybrid ranges from 2 to 3 times more than the LWR.

\section{Conclusions of the LLL/Bechtel Study}

The production of fissile fuel by a hybrid is a promising step in the development of fusion. This design study has shown that the addition of a depleted-uranium fission blanket to a laser-fusion system has several advantages:
The hybrid operates in a regime which requires an order of magnitude lower laser and pellet performance than a pure laser-fusion system.

- First-wall requirements and 14-MeV-neutron damage are less severe in a laser-fusion system with a fission blanket.

- The laser-fusion hybrid produces a large amount of fissile material-enough to fuel more than six LWRs of equivalent size.

When laser-fusion hybrids are producing fuel for existing reactors, the cost of electricity is insensitive to the capital cost of the hybrid.

- The laser-fusion hybrid would extend the total energy available from economical LWRs by two orders of magnitude.

The feasibiiity of the laser-fusion hybrid should be evaluated from three points of view: scientific, technological, and economic. The scientific feasibility of the laser-fusion hybrid is dependent on (1) achieving pellet gains in the neighborhood of 100 , and (2) developing suitable lasers with overall system efficiencies greater than $1 \%$.

The fission blanket surrounding the fusion chamber was designed with state-of-the-art fission technology to assess the technological feasibility of

Table 3-4. Fuel cycle cost bases for pressurizedwater and boiling-water fission and a hybrid reactor.

\begin{tabular}{|c|c|}
\hline Uranium & $s 40 / 16 \mathrm{U}_{3} \mathrm{O}_{8}$ \\
\hline Conweraion & $54.50 / \mathrm{kg} \mathrm{U}$ \\
\hline Enrichment & $\$ 100 / \mathrm{sw}$ \\
\hline Fabrication & $\begin{array}{l}5100 / \mathrm{kg} U \text { (PWR) } \\
580 / \mathrm{kg} U \text { (BWR) }\end{array}$ \\
\hline Spent-fuel thipping, & S $20 / \mathrm{kg} \mathrm{U}$ \\
\hline Reprocessing & $\$ 225 ; k g$ U \\
\hline Plutonium credit & $\begin{array}{l}\$ 34.25 / g \text { Puf(PWR) } \\
\$ 26.95 / g \text { Puf(BWR) }\end{array}$ \\
\hline
\end{tabular}

70\% plant capxity factor

17.4\% working copital change rate

$\begin{array}{lll}\text { Proces Losses: } & \text { Conversion } & 0.2 \% \\ & \text { Fabrication } & 0.5 \% \\ & \text { Reprocessing } & 0.5 \%\end{array}$

Luser-Fusion Hybris Reactor

$\begin{array}{lc}\text { Fabrication } & \$ 30 / \mathrm{kg} \mathrm{U} \\ \text { Spent-fued shipping } & \$ 10 / \mathrm{kg} \mathrm{U} \\ \text { Reprocessing } & \$ 125 / \mathrm{kg} \mathrm{U} \\ \text { Plutonium credit } & 330 / \mathrm{g} \mathrm{Pu} \text { f }\end{array}$


the laser-fusion hybrid. However, a definitive statement regarding the feasibility of fissile-fuel production with laser fusion cannot be made without further study. The laser and optical systems must be conceptually designed. The pellet manufacturing and injection systems need to be considered. A more detailed analysis of the first-wall design should be carried out. Finally, a safety analysis of the design is required, particularly of system failures which could result in a release of radioactive nuclides to the environment. The most obvious release mechanism is the melting of the fuel in a loss-uf-coolant accident.

The economic analysis shows that the cost of electricity in a hybrid-LWR scenario is insensitive to the capital cost of the hybrid. The laser-fusion reactor is estimated to be three times more expensive than an LWR, yet the cost of electricity will be only $40 \%$ more than the present price. Nevertheless, substantjal economic gains would be realized if the laserfusion hybrid costs could be decreased to twice that of an LWR. Possibilities for reducing capital cost that should be explored in future studies include:

Replacement of the coolant and tritjumbreeding systems with helium cooling and a solid lithium blanket.

- Investigation of fission blankets that enhance energy multiplication.

- Consideration of blanket geometries that more efficiently utilize the point source from laser fusion.

\section{Alternate Approaches Permitting A Long-Term Fission Economy}

Fission reactors operating on a throwaway uranium fuel cycle will exhaust their supply of economically accessible fuel in less than 100 years. Therefore, to extend the useful life of this economic source of electricity, the throwaway uranium fuel cycle must be replaced. Until the recent shift in government policy, the United States has concentrated on developing the LMFBR (which uses the plutonium fuel cycle), and on reprocessing spent reactor fuel to allow recycling of unburned $235 \mathrm{U}$ and Pu through LWRs. The President has introduced a program calling for indefinite ceferral of the reprocessing and recycling of plutonium and the acceleration of research into alternative nuclear fuel cycles.

Athough the fast breeder reactor involves a ful] commitment to the plutonium fuel cycle, hybrids do not require any fissile enrichment. Therefore, they can use uranium and thorium resources without reprocessing. To date, we have not examined these

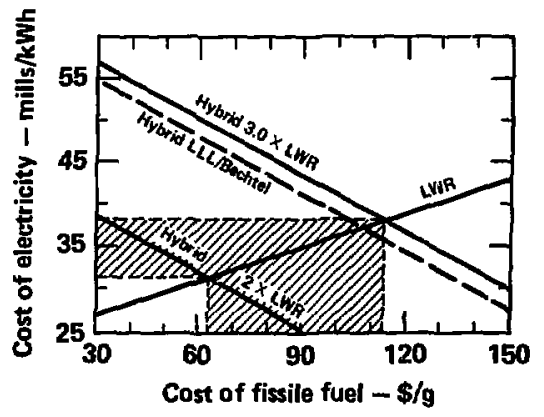

Fin. 3-7. Cost of flalle fuel and electricity from a hybrid. The intersection points of the curves detemine the cost of electricity and riadle fued in the hybrd-LWR senario. For axample, the cost of electricity increses by only 20 to $40 \%$ wher the capital cost of the hybrid ranges from two to three times more then the I.WR.

nonreprocessing options in any detail because, in general, they exhibit a lower fissile fuel production and result in more costly electricity than the reprocessing options. However, in light of the recent policy change, we briefly describe a few nonrecycling schemes for hybrids that appear plausible.

The most straightforward approach is to use hybrids strictly as power producers with throwaway fuel cycles. These hybrid power plants could be fueled with thorium, depleted uranium, or spent fuel from LWRs; therefore, they could take advantage of the fusion energy multiplying characteristics of fuels that are abundant and cheap. Hybrid systems will be significantly more expensive than LWRs. Hence, power-producing hybrids without fissile fuel revenues can be expected to generate electricity at substantially higher costs.

Another nonreprocessing scheme involves the fissile enriching of depleted uranium fuel by irradiation in a hybrid for subsequent burnup in an LWR. The feasibility of this approach primarily rests with the development of high-burnup fuel elements that could operate in both the hybrid and LWR environments. Alternatively, the enriched hybrid fuel could be declad, ground up, homogenized and compacted into LWR fuel pins. All of these operations would have to be conducted at high cost in shielded hot cells. Fissile fuel-producing hybrids utilizing either of these nonreprocessing schemes are expected to provide enough fissile material to fuel two LWRs of equivalent size. This represents a factor-of-three reduction in the fissile fueling capability that would be obtained with reprocessing. 
The ${ }^{233} \mathrm{U} /$ thorium cycle with reprocessing of spent fuel has been proposed as a "less proliferating" nuclear fission option. If so, thoriumfucled hybrids could advantageously produce ${ }^{233} \mathrm{U}$ for efficient-burning thermal fission reactors. In this approach, hybrids located in secure areas would supply fission hurner reactors with ${ }^{233} \mathrm{U}$ that has been denatured with ${ }^{238} U$ to one part in six. As a result, wcapons-grade material will not be present in the fuei supplied to the burner reactors. Because piutonium will be produced in the thermal fission reactors, the spent fuel must be returned to secure areas where the plutonium can be extracted and either utilized or stored. On the other hand, Cohen, in a recent presentation at the Atomic Industrial Forum Fuel Cycle '77, pointed out that denatured uranium is only about 12 separative work units away from weapons-grade material and that a crude centrifuge plant costing about 4 million dollars could be used to produce enough fissile material for a weapon in less than six weeks.

\section{References}

1. Laser Program Annual Report-1976, Lawrence Livermore Laboratory, Rept. UCRL-50021-76 (1977).

2. Laser Program Annual Report-1975, Lawrence Livermore Laboratory, Rept. UCRL-5002k75 (1976).

3. Laser Fusion Hybrid Reactor Systems Study, Bechtel Corporation (1976).

4. J. Hovingh, Design Consideration in Inertially Confined Fusion Reactors, Lawrence Livermore - Laboratory, Rept. UCRL-78499 (1976).

5. J. Hovingh, "First Wall Studies of a LaserFusion Hybrid Reactor Design," Proc. 2nd Topical Meeting on the Technology of Controlled Nuclear Fusion. Vol. II, 765, (Richland, Washington, 1976).

6. G. B. Zimmerman, Numerical Simulation of the High Density Approach to Laser Fusion, Lawrence Livermore Laboratory, Rept. UCRL-748 I1 (1973). 


\section{ACTINIDE BURNING}

The neutrons from DT-fusion systems can change long-lived fission waste products (primarily actinides) to short-lived or stable isotopes. This method could serve as an alternative to geological storage of the long-lived radioactive wastes. Actinide half-lives are quite long, even in comparison to such geological phenomena as ice ages and earthquake fault movement. Storage of long-lived wastes is difficult because these events can render a disposal area unacceptable.

If fission wasle products are allowed to decay naturally, actinides will comprise most of the radioactive hazard after 400 years. Thus, if the actinides can be transmuted to stable isotopes, or fissioned into relatively short-lived products, storage time can be reduced substantially.

Studies have investigated this process in a variety of reactor types, and we believe that the most effective means of reducing the long-term radiotoxicity of actinides is depletion by fissioning the actinides. This is most effectively accomplished in an inertially confined fusion reactor. However, problems do exist in this method, and perhaps the inertially confined fusion reactors can be put to better use.

Several studies have investigated the possibility of recycling actinide wastes in thermal fission reactors 1 and fast breeder reactors. ${ }^{2}$ These studies have shown that it is possible to burn actinides in fission reactors and that the process is more efficient in the harder neutron spectra of fast breeder reactors. Logically, fast-neutron-rich fusion systems could even more effei:ively serve the same role.

\section{Actinide Burning In Fusion Reactors}

Two studies of actinide burning in fusion reactors have been completed. Battelle Northwest Laboratory (BNWL) performed a study of actinide burning in a beryllium blanket with a first-wall fusion neutron flux of $10 \mathrm{MW} / \mathrm{m} 2$ and an irradiation time of 500 days. 3 Battelle concluded that the firstwall loading was an order of magnitude too small for effective transmutation in the moderated neutron spectrum of the beryllium blanket.

Another study recently completed by the Fusion Power Division of Westinghouse (WFPS) investigated actinide burning in a fast spectrum. Irradiation time in the conceptual study was 30 $y$ cars and first-wall fluxes were $1.15,5$, and 10 $\mathrm{MW} / \mathrm{m}^{2}$ in a Tokamak configuration. ${ }^{4}$ The WFPS reactor blanket produces $13,000 \mathrm{MW}_{\mathrm{t}}$ of power. For a neutron flux of $1.15 \mathrm{MW} / \mathrm{m}^{2}$, the hazard index initially decreased. At $10^{5}$ years, however, it increased back to the natural decay level as shown in Fig. 4-1. These results matched those of the BNWL study. For the largest neutron first-wall tlux, 10 $\mathrm{MW} / \mathrm{m}^{2}$, the hazard index wus reduced by three orders of magnitude in 100 vears (Fig. 4-2).

\section{WFPS Study 4}

The EPRI-sponsored Westinghouse study was based on a beam-driven Tokamak fusion-fission hybrid reactor used for actinide deplction. Intended for mid-to-late 1980's technology, this reactor provides a fusion neutrin wall loading of approximately $1 \mathrm{MW} / \mathrm{m}^{2}$ corresponding to approximately $10^{14} \mathrm{r} / \mathrm{cm}^{2} . \mathrm{s}$ in the $14-\mathrm{MeV}$ energy range. The fission blanket is fueled with residual actinides contained in the high-level waste from the LWR-U cycle. This fuel is primarily comprised of ${ }^{237} \mathrm{~Np}$, ${ }^{241} \mathrm{Am}$, and ${ }^{243} \mathrm{Am}$ which represent almost $90 \%$ of the residual actinides and are the principal contributors to the very long-term radioactive hazards in LWR waste. These residual actinides are the only fissionable material contained in the helium-cooled blanket, but they provide sufficient neutron multiplication for neutron fluxes in the range of $10^{15}$ $\mathrm{n} / \mathrm{cm}^{2} \cdot \mathrm{s}$.

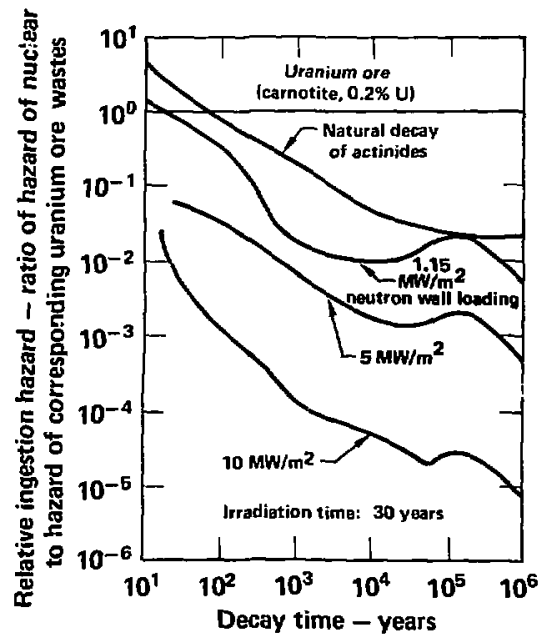

Fig. 4-1. A compurison of the relative ingestion hazards from aminides as functions of decay time. 


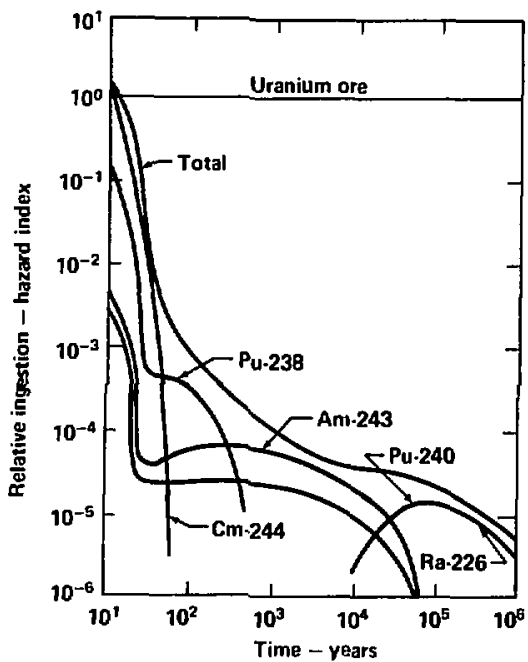

Fig. 4-2. The effect of irradiation with a $10 \mathrm{MW} / \mathrm{m}^{2}$ fusion 'neutron wall loading on the long-term ingestion $b= \pm=-d$ potential of IWR actinides following 30 years of irradiation in the nctinide bumer.

Several radiotoxic hazard indices have been commonly used in connection with nuclear-safety and nuclear-health analyses. These are the ingestionhazard index, the inhalation-hazard index, and the radiation-dose rate. Early studies on nuclear-waste management problems have generally used the ingestion hazard as the index for evaluating relative toxicities, primarily because ingestion is the most relevant long-term hazard. However, the absolute magnitude of the ingestion-hazard index has little practical meaning when taken out of the context of specific safety analyses that involve waste-release mechanisms. Computation of the absolute value of the ingestion hazard becomes somewhat meaningless. Therefore a relative index was developed for use in the actinide-burner study. This index consists of the ratio of the ingestion hazard of a given amount of high-level nuclear waste to the ingestion hazard of the amount of naturally-occurring parent uranium ore from which the wastes were produced. Formulation of a relative hazard index on this basis is considered to be a more realistic measure of the effects of high-level wastes because it compares the hazard potential of wastes with that of naturally occurring radioactive substances which would exist if the uranium was not used in the LWR.
The evaluation of the effect of approximately 1 $\mathrm{MW} / \mathrm{m}^{2}$ fusion neutron wall loading on actinide depletion showed that the principal residual actinides from fission reactors can be depleted effectively using fusion neutrons. The rates of depletion for ${ }^{237} \mathrm{~Np},{ }^{243} \mathrm{Am}$, and ${ }^{241} \mathrm{Am}$ are found to be nearly exponential; consequently, a convenient way to compare their rates of depletion with the rates of natural decay is to define an "effective half-life." This is the half-life of the isotope during the time it is irradiated in the actinide burner. The effective half-lives of these three principal actinides during a 30-year irradiation are several orders of magnitude shorter than their natural half-lives. Therefore, it seems that the irradiation has appreciably improved the very long-term radiotoxic hazard potential. However, the initial actinide isotopes are significantly depleted by transmutations (neutron absorptions) even in the very hard neutron spectrum of the actinide-burner blanket, and significant amounts of ${ }^{238} \mathrm{Pu},{ }^{244} \mathrm{Cm}$, and ${ }^{242} \mathrm{Cm}$ are produced. These are eventually depleted with extended irradiation, but the net effect of the production of ${ }^{238} \mathrm{Pu}$ and ${ }^{244} \mathrm{Cm}$ is a significant increase in the hazard index over the first few years of irradiation. This results from the relatively shorter half-lives of ${ }^{242} \mathrm{Cm},{ }^{244} \mathrm{Cm}$, and ${ }^{238} \mathrm{Pu}$ when compared to the other actinides. Additionally, the ${ }^{242} \mathrm{Cm} /{ }^{238} \mathrm{Pu}$ decay chain gives rise to very radiotoxic long-term daughter products. In fact, all the actinides associated with fission reactor wastes will create a dozen or so radioactive daughter products before decaying to stable $\mathrm{Pb}$ or Bi isotopes. These considerations provide the following conclusions with regard to the actinides:

- In a meaningful evaluation of the long-term effects on radiotoxicity, one must follow the decay chains for approximately $10^{6}$ years to see the effects of radioactive daughter products on the potential ingestion hazards.

- Depletion of any actinide isotope by transmutation (i.e., neutron absorption) only forms another actinide with a different set of long-term radioactive daughter products. This transmutation does not necessarily reduce the long-term radiotoxic-hazard potential; in fact, for transmutation to ${ }^{238} \mathrm{Pu}$, a significant increase is noted.

- Therefore, the most effective means of reducing very long-term radiotoxicity from the actinides is depletion by fission.

Since successive neutron reactions (e.g., transmutation followed by fission) are required to convert the majority of the actinides to suitably short-lived fission products, the total fluence necessary for effective actinide depletion is quite high. Thus, the 
reference case utilizing a fusion neutron wall loading of $1.15 \mathrm{MW} / \mathrm{m}^{2}$ is only marginally attractive, reducing the long-term hazard potential from actinides by (at most) one order of magnitude after 30 years irradiation.

In view of the above results, the effect of a postulated increase in fusion neutron wall loading was examined. The results showed that $5 \mathrm{MW} / \mathrm{m}^{2}$ of neutron wall loading will reduce the ingestion hazard by one order of magnitude compared to natural decay. A neutron wall loading of 10 $\mathrm{MW} / \mathrm{m}^{2}$ can reduce the long-term ingestion hazard by three orders of magnitude. Blanket nuxes of approximately $10^{15} \mathrm{n} / \mathrm{cm}^{2} \cdot \mathrm{s}$ of very high-energy (10to (5-MeV) neutrons are generated in the latter case. Neutrons in this energy range primarily cause fission reactions rather than neutron absorptions. This, coupled with the increased value of $\nu$ (neutrons released per fission) for very high-energy neutrons, gives a significant increase in blanket neutron multiplication for a given amount of fuel. Therefore, a substantial reduction in actinide loading density (by a factor of about four) and a corresponding increase in the high-energy fraction of the neutron flux can be realized while retaining the same blanket power density. This hardening of the neutron flux improves the depletion and results in a decrease in the effective half-lives of all the actinides. For example, the effective half-life of ${ }^{237} \mathrm{~Np}$ decreases from 4.5 years to 1.5 years and that of ${ }^{238} \mathrm{Pu}$ from approximately seven years to two years for the $1.15 \mathrm{MW} / \mathrm{m}^{2}$ case and $10 \mathrm{MW} / \mathrm{m}^{2}$ case, respectively.

The ultimate capability of a beam-driven Tokamak appears to yield a wall loading on the order of 4 to $5 \mathrm{MW} / \mathrm{m}^{2}$, if one can achieve an elongated plasma $(b / a=3)$ and if close to $1000 \mathrm{MW}$ of netural-beam power can be injected into the plasma. At present, laser fusion appears to be capable of attaining a $10-\mathrm{MW} / \mathrm{m}^{2}$ fusion neutron wall loading. Another possible alternative would be actinide depletion in fission reactors. Blanket neutronic results from the fusion-driven actinide burner study suggest that it may be possible to develop a fast helium-cooled fission reactor using only residual actinides from high-level wastes as fuel. However, fusion neutrons appear to have a distinct advantage over recycling in fission reactors if fusion neutron wall loadings approaching 10 $\mathrm{MW} / \mathrm{m}^{2}$ are available.

The preceding discussion on actinide depletion is based on the premise that all actinide isotopes that contribute to the hazard index are undesirable nuclides. On the other hand, ${ }^{238} \mathrm{Pu},{ }^{244} \mathrm{Cm}$, and ${ }^{242} \mathrm{Cm}$ have identifíable commercial applications as beneficia! radioisotopes. For example, ${ }^{238} \mathrm{Pu}$ is useful for portable and remote power sources that include pacemakers, artificial hearts, ard space and terrestrial power stations. The principal deterrent to the use of these nuclides has been their unavailability and high cost. The actinide burner may be a prolific source of these radioisotopes, which are produced by actinide transmutation. The ${ }^{238} \mathrm{Pu}$ isotope is the principal contributor to the increase in the hazard index during irradiation. and its daughter product, ${ }^{226} \mathrm{Ra}$, is largely responsible for a secondary peak in the hazard index at approximately $10^{5}$ years (Fig. 4-2).

Thus, a value judgment is needed to weigh the beneficial and potentially detrimental attributes of nuclear materials. In fact, Fig. 4-1 suggests that, in terms of the relative hazard index defined earlier, even unirradiated actinides represent a comparatively small long-term radiotoxic hazard. In view of these considerations and recent studies of waste transport into the ecosystem, the issue of long-term hazard potential from actinides should be reexamined.

Material requirements for the actinide burner indicate that radiation damage is most significant for the liner and vacuum-vessel wall. Bulk radiation damage, rather than surface damage, is the principal concern for the vacuum-vessel wall. Analysis indicates that stainless steel is adequate for the vacuum vessel if temperatures are limited to $450^{\circ} \mathrm{C}$. However, the effects of radiation on weld fusion zones and zones heated during vacuum-vessel fabrication must eventually be evaluated. The effect of the hefium production on swelling is also a concern that will ultimately require further evaluation. Presently the goal is to design the vacuum-vessel wall to operate five years before replacement. Liner materials are even more difficult to protect from radiation damage because both surface and bulk radiation effects must be considered. In view of these considerations, Mo-TZM* is usually selected for the liner because it meets the liner structural requirements, assuming the diverter operates as effectively as predicted and that the liner temperature can be limited to approximately $700^{\circ} \mathrm{C}$. The severe temperature and radiation environment preclude the choice of stainless steel for the liner. The design goal for the liner is a two-year operation before replacement. This goal is reasonable for a demonstration reactor considering the present primitive knowledge of material behavior under fusion neutron irradiation. Longer design lifetimes may be required for commercial plants if the

\footnotetext{
-Aл alloy of molydenum, titanium, and zirconium.
} 
replacement times of these components are excessive.

The WrPS study has shown that, from the standpoint of economics, the actinide burner can produce a very large amount of useful energy (approximately $13,000 \mathrm{MW}_{1}$ ). Moreover, this energy can be supplied at conditions of fluid temperature and pressure suitable for useful power generation. The plant WFPS used with the actinide burner provides $1050 \mathrm{MW}_{\mathrm{u}}$ for its required circulating power, as well as a surplus of arproximately 10,000 $M W_{1}$ for useful export. In view of the extremely large power of the plant, it was not considered reasonable to supply this export power in the form of electricity. Instead, a hydrogen-production plant was coupled to the reactor to create a marketable product from its power. The total capital cost for the reactor and plant (excluding escalation and in. terest during construction) was estimated to be approximately $\$ 4.7$ billion (in 1975 dollars). On the basis of capital costs per unit of thermal output, this figure is approximately three times that of an LWR. Assuming a ter-year design and construction period with operation in the mid-1980's (which is extremely optimistic), provision for escalation would require $\$ 4.6$ billion and the interest during construction another $\$ 3.9$ billion, a total plant cost of $\$ 13.2$ billion. If one examines operating costs, including amortization of the \$13.2-billion investment over a 30-year plant life and crediting the hydrogen production at the same price per Btu contents as that for oil, there is a net operating deficit of \$1.9 billion per year which can be assigned as the incremental cost for disposing of the actinide inventory. To put this cost in perspective, the actinide burner has the capability to fission 4 metric tons of actinide per year, based on a power generation of $13,000 \mathrm{MW}_{1}$ with an availability of $80 \%$. Since depletion of these actinides by fission corresponds to disposal of fission-reactor actinide waste produced in the generation of $2.2 \times 10^{11} \mathrm{kWh},{ }^{*}$ an $8.5 \mathrm{mills} / \mathrm{kWh}$ incremental cost can be assigned to the fission-reactor power plant for actinide burning. This is about a $20 \%$ increase in the total generation cost for a nuclear plant scheduled for 1985 start up. However, the actinide burner concept has not been optimized, and significant cost improvements may be feasible.

Major development programs will be required for many new technologies to support the design and construction of the actinide burner. Although crash efforts might have actinide burners operating by 1990, the probability of all the requisite

\footnotetext{
*Thirty-three plants ( $1 \mathrm{GW}_{\mathrm{e}}$ cach) operating at a $75 \%$ capacity factor produce $2.2 \times 10^{11} \mathrm{kWh} / \mathrm{yr}$.
}

technologies being available on this time schedule is rather unlikely.

The environmental and safety considerations associated with the actinide burner pose a variety of new problems, although there are parallels with present !ission reactor plants. Perhaps the most significant environmental constraint is the need to contain a very large inventory $(300 \mathrm{MCi})$ of tritium. This inventory is several orders of magnitude larger than that contained in current fission reactor plants. Thereforc, features such as multiple containment and cleanup systems must be carefully designed. With regard to safety considerations, $13,000 \mathrm{MW}$ is more than three times the $3800 \mathrm{MW}$, eurrently imposed as an upper limit for LWRs. Furthermore, the actinide-fuel inventory represents a very large collection of those components of high-level radioactive wastes having the most adverse longterm toxicity characteristics. Thus, we anticipate that a serious program to design, develop, and construct such it plant would have to include provision for an extensive review of the regulatory implications. The principal consideration in the $3800 \mathrm{MW}$, limit is the desire to obtain operating experience with reactors up to that size in a comparatively mature LWR technology. On the other hand, a single actinide burner of the type described in this study would service more than thirty LWR's and would not be restricted by siting constraints that tend to favor location near electrical load centers.

\section{Actinide Burning in an Inertially Confined Fusion (ICF) Reactor}

ICF reactors will be more effective actinide burners than magnetically confined fusion systems. They may be able to operate with higher first-wall loadings than beam-driven, magnetically confined fusion reactors, and the absence of magnets will allow more flexibility in the blanket design.

Berwald and Duderstaqi have proposed a laserdriven fusion reactor to burn actinide wastes. 5 Their reactor, based on the $W$ isconsin laser-fusion reictor design, is expected to process about 7.6 tonnes per year of actinide wastes in the blanket at equilibrium, ${ }^{*}$ which corresponds to the actinides produced by $136 \mathrm{LWRs}$ ( $1 \mathrm{GW}$ each) operating at a $75 \%$ capacity factor. These actinides, in the form of actinide nitride precipitate mixed in an actinideliquid tin alloy, are continuously circulated in the blanket providing uniform exposure of the charge.

\footnotetext{
-Equilibrium is reached in the seventh year of operation. Prior 10 equilibrium, the renctor operates at different pulse rates, wall londings, and actinide burn rates. A peak will loading of 10 $\mathrm{MW} / \mathrm{m}^{2}$ occurs at stortup when the pulse rate is $41 \mathrm{~s}^{-1}$.
} 
The fusion yield is $100 \mathrm{MJ}$ and the pulse rate of the reactor is $20 \mathrm{~s}^{-1}$ for a fusion power of $2000 \mathrm{MW}$. The first-wall neutron flux is $5 \mathrm{MW} / \mathrm{m}^{2}$, and the specific power in the actinide-fueled blanket is about $300 \mathrm{MW} / \mathrm{m}^{3}$. The total power of the system is about $22,000 \mathrm{MW}$. The actinide nitride-liquid-tin fuel processing technique proposed by Berwald and Duderstadt is based on work by Anderson and Parlee. ${ }^{6}$

An additional technigue for burning actinide wastes in an ICF reactor is to burn the actinides in a fusion fuel pellet. This technique increases the neutron fuence per shot in the actinides by a factor greater than $10^{9}$. Calculations have shown that most of the actinide materials seeded into the peliet can be burned. The disadvantage of burning the actinide in pellet form is that pellet fabrication and processing of fission product waste becomes more complicated. This process is important because a given mass of actinjdes will need to be reprocessed several times before it is completely fissioned. In addition, the rate of actinide mass burned in pellets will be much less than that in a blanket because there is a limit to the pellet mass that can be compressed by a given ignitor-beum energy.

\section{Conclusions}

The most effective means of reducing the long term radiotoxicity from the actinides is depletion by fissioning the actinides. To effectively burn actinides in a fusion reactor, the product of irradiation time and first-wall fusion neutron flux must be on the order of $300 \mathrm{MW} y r / \mathrm{m}^{2}\left(10^{10} \mathrm{MJ} / \mathrm{m}^{2}\right)$. The $\mathrm{fu}$ sion reactors that burn actinides will be very large with power levels from 10 to $50 \mathrm{GW}_{\mathrm{t}}$. Because of their size, they will generate too much power to be tied 10 a grid. The excess energy can be used for hydrogen production (see Section 5) by electrolysis or for desalinization of water to help reduce the in. cremental cost assigned to LWR power plants for waste disposal.

It appears that the ICF reactor will be more attractive for actinide-busning applications than alternative systems ( $L W R, L M F B R$, and magnetically confined fusion). However, the actinide-burning process is of questionable desirability for the following reasons:

- Compared to immediate encapsulation and storage of actinides, increased handling of highlevel wastes is required.
- The short-term hazards increase during early irradiation becausc of the production of additional actinides from neutron capture.

- Some actinides isotopes are quite useful and should not be burned.

- Reduction of effective half-life from the natural half-life for actinides is of questionable value. Even without irradiation. the actinides present the same ingestion hazard as the parent ore after a relatively short storage period (100 years).

If socie' $v$ decides that the long-term radiological hazards lron, fission-reactor actinide wastes are high enough to merit construction of actinide burners, then inertially confined fusion technology cun best perforn the required irradiation.

\section{References}

1. H. C. Claiborne, Noutron Induced Transmutation of IVigh Level Radioactive Waste. Oak Ridge National Laboratory, Oak Ridge, Tennessee, ORNL-TM-3964 (1972).

2. S. L. Beuman and E. A. Aitken, "Feasibility Studies of Actinide Recycle in LMFBR's as a Waste Management Alternative," American Nuclear Society Summer Amual Meeting (Toronto, Canada, (1976).

3. U. P. Jenquin and B. R. Leonard, "Actinide Transmutation in Fusion Reactor Blankets," American Nuclear Society Summer Amual Nieting (Toronto. Canada, 1976).

4. R. P. Rose et al., Fusion-Driven Actinide Burner Design Situd!: Vol. I, EPRI contract RP-473-I. Westinghouse Fusion Power System Dept., Westinghouse Electric Corporation. WFPSTME-033 (1976).

5. D. H. Berwald and J. J. Duderstadt, Preliminary Design and Neutronic Analy'sis of A Laser-Fusion Driven Actinide Waste Burning Hybrid Reactor, Dept. of Nuclear Engineering. The University of Michigan, Ann Arbor. Michigan, Rept. 015189 (October 1977).

6. R. N. Anderson and N. A. D. Parlee, "Continuous Removal of Fission Products in a Nitride-Fueled Reactor," Nud. Techmol. 13, 300 (1972). 


\section{SYNTHETIC FUEL PRODUCTION}

Our oil and natural-gas supplies are declining rapidly. Hence, the ability to produce large quantities of portable chemical fuels for our homes, industries, and vehicles will be a necessity within the next 100 years. Natural gas has been the preferred fuel for residential heating and industrial-process heat; it is environmentally acceptable, convenient to use, and, until recently, low in cost and in ample supply as a natural resource. Depletion of natural reserves has added emphasis to the search for alternative fuels. The role of hydrogen, both as a fuel and as an intermediate product used in the production of synthetic fuels, is being considered. An example of synthetic fuel production is the reduction of both carbon and carbon monoxide by hydrogen at high temperatures to form methane $\left(\mathrm{CH}_{4}\right)$. Methane is the principal constituent (typically about $85 \%$ ) of natural gas.

Any large-scale, long-range scheme for the production of synthetic fuels should not require further depletion of fossil-fuel reserves. It is inconsistent, for example, to use natural gas when producing hydrogen for use as a substitute fuel. However, the abundance and general availability of water make it an ideal feedstock for hydrogen production processes. A laser-fusion reactor could supply the energy necessary to decompose the water, and such a system would represent a virtually inexhaustible source of hydrogen.

Depending on how energy from the fusion reaction is used; water-cracking processes can be classified as thermochemical, electrolytic, or radiolytic. Figure S-1 compares the various conversion processes, on the basis of recoverabie hydrogen-combustion energy per unit of thermal energy available.

The thermal energy from the reactor could support a sequence of high-temperature chemical reactions in various hydrogen-producing thermochemical processes. The system of reactions known as the Mark-1 cycle is typical of the processes involved: '

$\mathrm{CaBr}_{2}+2 \mathrm{H}_{2} \mathrm{O} \stackrel{730^{\circ} \mathrm{C}}{\longrightarrow} \mathrm{Ca}(\mathrm{OH})_{2}+2 \mathrm{HBr}$

$\mathrm{Hg}+2 \mathrm{HBr} \stackrel{250^{\circ} \mathrm{C}}{\longrightarrow} \mathrm{HgBr}_{2}+\mathrm{H}_{2}$

$\mathrm{HgBr}_{2}+\mathrm{Ca}(\mathrm{OH})_{2} \stackrel{200^{\circ} \mathrm{C}}{\longrightarrow} \mathrm{CaBr}_{2}+\mathrm{HgO}_{\mathrm{gO}}+\mathrm{H}_{2} \mathrm{O}$

$\mathrm{HgO} \stackrel{600^{\circ} \mathrm{C}}{\longrightarrow} \mathrm{Hg}+(1 / 2) \mathrm{O}_{2}$.
The net process is the decompesition of water

$$
\mathrm{H}_{2} \mathrm{O} \longrightarrow \mathrm{H}_{2}+(1 / 2) \mathrm{O}_{2}
$$

The maximum temperature required $\left(730^{\circ} \mathrm{C}\right)$ could be achieved in a gas-cooled laser-fusion reactor.

A large number of alternate chemical processes could ultimately produce hydrogen and oxygen from water. While these thermoshemcial processes generally do not consume the chemical reactants, they often use large quantities of hazardous and corrosive chemicals. Practical energy-conversion efficiencies for typical thermochemical processes range from about 30 to $65 \%$. 1,2 In other words, up to $65 \%$ of the fusion energy is recoverable as thermal energy by burning the hydrogen produced.

Laser-fusion reactors could also generate electricity for subsequent electrolysis of water to produce hydrogen. The electricity simply supplies the energy required to decompose water according to

$$
\mathrm{H}_{2} \mathrm{O}(\mathrm{l}) \longrightarrow \mathrm{H}_{2}(\mathrm{~g})+(1 / 2) \mathrm{O}_{2}(\mathrm{~g})
$$

This reaction absorbs $68 \mathrm{kcal}$ per mole of hydrogen produced. For existing electrolysis plants, the combustion energy of the hydrogen produced is 60 to $100 \%$ of the electrical energy input. ${ }^{1,3}$ Assuming a $40 \%$ thermal-to-electric conversion efficiency for the laser-fusion power plant, the electrolysis process produces hydrogen with an overall energyconversion efficiency of 24 to $40 \%$.

Hydrogen production by radiolysis can be achieved in several ways, but all require an intense source of radiation. The penetrating neutron radiation of a laser-fusion reactor could be utilized for radiolysis by incorporating sufficient quantities of $\mathrm{H}_{2} \mathrm{O}$ in the blanket regions surrounding the fusion vacuum chamber. 2,4

A concept often used in the discussion of radiation-induced chemcial processes is the $G$ value. The $G$ value for the formation of a chemical species is defined as the number of molecules produced per $100 \mathrm{eV}$ of energy deposited in the production regions by incoming radiation. The generally accepted $\mathrm{G}$ value for $\mathrm{H}_{2}$ from water is 0.45 , while $\mathrm{G}$ values as high as $\mathbf{1 3 . 6}$ have been measured in experiments using pure steam exposed to high-energy neutrons. ${ }^{4}$ Radiolytic hydrogen production is clearly more efficient in steam than in liquid water. 
Fig. 5-7. The combustion energy of hydrogen recoverable from one unit of fusion-reactor therwal energy. The fraction of the weutron energy deposited in hydrogen processing regions is generoudy assuned to be $100 \%$ for radiolyss of water and $60 \%$ for radiolysis of steam. It is ualikely that dedicated radiolysis hydrogen-production facility could ever prove practical unless a liquid medium with a higher 6 ralue is found. Therefore, a hypothetical $G=20$ liquid medium system is shown.

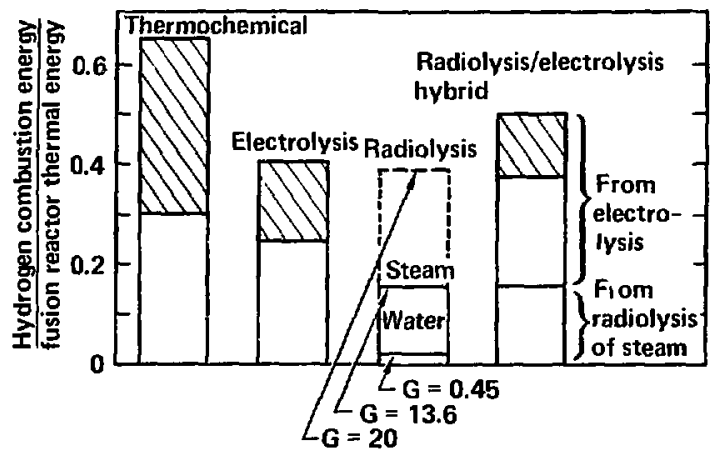

Figure 5-2 is a power-flow diagram for a laserfusion hydrogen-production plant that also generates electricity. Note that more than $25 \%$ of the energy released by the fusion microexplosion is carried by the pellet debris, alpha particles, and $x$ rays. This energy is deposited in the vacuum chamber wall and thus is not available for radiolytic decompositjon.

Figure 5-2 also shows that $P_{n}$ is the total energy deposited in the reactor as a result of neutron interactions with blanket materials. It is important to note that only a fraction, $\alpha$, of the available neutron energy is actually deposited in the steam blankets; the remainder is deposited in reactor structural materials and thus is unavailable for radiolysis of $\mathrm{H}_{2} \mathrm{O}$. For example, less than $40 \%$ of the fusion neutron energy would be deposited in a 3-m-thick region of pressurized steam at 1000 psia. Not all of the nuclear energy deposited in the steam is used in subsequent radiolysis and $\mathrm{H}_{2}$ production. The term $\eta_{H}$ is the conversion efficiency of nuclear energy deposited in the steam to potential combustion energy of the hydrogen produced. It is related to the $G$ value for the formation of $\mathrm{H}_{2}$ by

$$
\eta_{H}=\frac{G h}{100}
$$

where $\mathrm{h}=$ heat of combustion of an $\mathrm{H}_{2}$ molecule $=$ $2.5 \mathrm{eV}$.

The primary reaction in the radiolysis of water vapor is the breaking of $\mathrm{O}-\mathrm{H}$ bonds to give $\mathrm{H}, \mathrm{O}$, and $\mathrm{OH}$ radicals. The minimum energy required to break the first $\mathrm{O}-\mathrm{H}$ bond in $\mathrm{H}_{2} \mathrm{O}$ is $5.2 \mathrm{eV}$, whereas the net energy consumed in the production of an $\mathrm{H}_{2}$ molecule from $\mathrm{H}_{2} \mathrm{O}(\mathrm{g})$ is equal to the heat of combustion of $\mathrm{H}_{2}$ and is only $2.5 \mathrm{eV}$. The difference results from subsequent exoergic $\mathrm{H}_{2-}$ and $\mathrm{O}_{2-}$ producing reactions. Because the chemical energy released in these reactions is ineffective in breaking additional $\mathrm{H}_{2} \mathrm{O}$ molecules, the conversion efficiency of nuclear energy deposited to usable chemical energy (combustion heat of $\mathrm{H}_{2}$ ) is less than unity $(100 \%)$. If a radiolytic reaction requires the minimum $5.2 \mathrm{eV}$ to break a single $\mathrm{O}-\mathrm{H}$ bond in $\mathrm{H}_{2} \mathrm{O}$, and the resulting $\mathrm{H}$ and $\mathrm{OH}$ radicals chemically react by

$$
\begin{aligned}
& 2 \mathrm{H} \rightarrow \mathrm{H}_{2} \\
& 2 \mathrm{OH} \rightarrow \mathrm{H}_{2}+\mathrm{O}_{2},
\end{aligned}
$$

then $2.5 \mathrm{eV}$ of potential combustion energy is produced from $5.2 \mathrm{eV}$ of nuclear energy. Hence, the maximum value of $\eta_{H}$ is about $48 \%$.

The maximum experimental G-value of 13.6 results in a value of $\eta_{H}=0.34$. In other words, $34 \%$ of the energy deposited in the steam is converted to potential hydrogen combusion energy while $66 \%$ appears as thermal energy in the system. The total fraction of $P_{n}$ that is converted into thermal energy is

$$
(1-\alpha)+\left(1-\eta_{\mathrm{H}}\right) \alpha=1-\eta_{\mathrm{H}} \alpha .
$$

The product $\eta_{H^{\alpha}}$ is an indication of the system efficiency for hydrogen power production, reflecting not only the efficiency in converting deposited nuclear energy to hydrogen but also the efficiency at which this energy is deposited in the hydrogenproducing regions. The overall energy-conversion efficiency is the ratio of the potential combustion energy of produced hydrogen to the total nuclear energy deposited in all regions of the reactor. As 


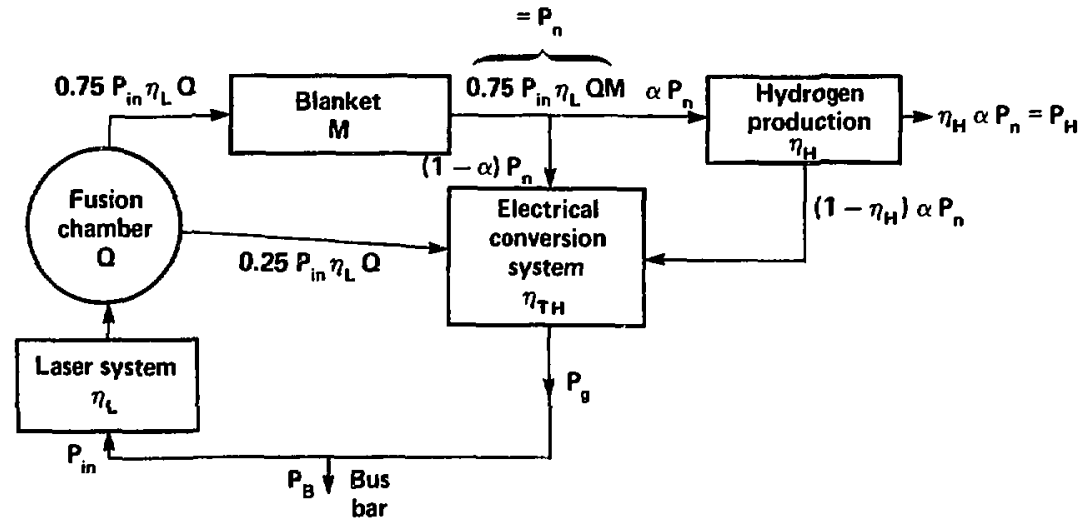

$$
\begin{aligned}
& \alpha=\text { fraction of nuclear energy deposited } \\
& \eta_{\mathrm{H}}=0.01 \mathrm{Gh} \text { is the conversion efficiency } \\
& G=\text { number of } \mathrm{H}_{2} \text { molecules produced } \\
& h=\text { heat of combustion of an } \mathrm{H}_{2} \text { molecule } \\
& =2.5 \mathrm{eV} \\
& \mathbf{P}_{\mathrm{H}}=\text { hydrogen combustion energy produc- }
\end{aligned}
$$

Fig. 5-2. The power flow diagram for a laser fusion radiolysis facility which also generates electricity.

shown in Fig. 5-2, the combustion energy of hydrogen produced is

$$
P_{H}=x_{1,} \propto P_{n},
$$

while the total energy deposited is

$$
P_{T}=P_{n}+0.25 P_{\text {in }} \eta_{L} Q=P_{n}+\frac{P_{n}}{3 M}
$$

The overall energy-conversion efficiency is then

$$
\eta=\frac{\mathbf{P}_{H}}{\mathbf{P}_{\mathrm{T}}}=\eta_{\mathrm{H}^{\alpha}}\left(\frac{3 \mathrm{M}}{3 \mathrm{M}+1}\right) .
$$

The blanket energy multiplication $M$ can be increased above 1.0 by adding boron to the steam blankets. Thermal neutrons absorbed by ${ }^{10} \mathrm{~B}$ release $2.35 \mathrm{MeV}$ of high LET radiation according to the reaction

$$
{ }^{10} \mathrm{~B}+\mathrm{n} \rightarrow \alpha+{ }^{7} \mathbf{L i}+2.35 \mathrm{MeV} .
$$

Thus, values of $M$ as high as 1.2 might be possible.

Using the values of $\eta_{H}=0.34$ (corresponding to $\mathrm{G}=13.6$ in steam), $M=1.2$, and generously assuming $\alpha=0.6$ for a steam blanket, the overall energy-conversion efficiency is only $16 \%$. Liquid water blankets have much lower efficiencies (even though $\alpha$ may be neasly unity) as a result of the very low $\mathbf{G}$ value of 0.45 . This gives a value of $\eta_{\mathrm{H}}=0.01$, and for $\alpha=1$ results in an overall energyconversion efficiency of less than $0.8 \%$.

If tritium-breeding zones are required, they would reduce the fraction, $\alpha$, of neutron energy available for hydrogen production. Because lithium is vigorously reactive with water, special precautions would be required to reduce the potential hazard. Also, because boron absorbs the neutrons required to breed tritium, it could not be used as an energy multiplier. A net reduction in the overall energy conversion efficiency for radiolysis results when tritium breeding requirements are considered. 
Therefore, while radiolytic hydrogen production by a laser-fusion reactor may be technically feasible, it is unlikely that a radiolysis facility using $\mathrm{H}_{2} \mathrm{O}$ could prove practical. Near term R\&D efforts in neutron radiolysis should concentrate on chemical schemes that could yiejd much higher $\mathbf{G}$ values, preferably in liquid media. Chemicals with hydrogen bond energies less than those in $\mathrm{H}_{2} \mathrm{O}$ would be likely candidates, and the use of a liquid would offer more efficient energy absorption than a gaseous medium. For example, with a $G$ value of 20 in a liquid medium, laser-fusion radiolysis would have an overall energy conversion efficiency of $37.5 \%$, which is competitive with the alternative processes for producing hydrogen in a laser fusion reactor (see Fig. 5-1).

An option for the water-based radiolysis system would be the use of the remaining thermai energy ( $585 \%$ of the total) for the production of electricity. The electricity could be sold on the electric power grid or used to generate more hydrogen by electrolysis. The overall energy-conversion efficiency of a radiolysis/electrolysis hybrid system is given by the sum of the overall radiolysis efficiency and the overall electrolysis efficiency applied to the remaining thermal energy; i.e.,

$\eta=m_{H}\left(\frac{3 M}{3 M+1}\right)$

$$
+\eta_{E} \eta_{T h}\left[1-\alpha \eta_{H}\left(\frac{3 M}{3 M+1}\right)\right] \text {. }
$$

where $\alpha, \eta_{\mathrm{H}}, \mathrm{M}$ are defined as before, and

$\eta_{\mathrm{L} .} \quad=$ electric-to-hydrogen conversion efficiency

$\eta_{\mathrm{Th}}=$ thermal-to-electric conversion efficiency

Thus, when the overall radiolysis conversion efficiency is $16 \%$,

$$
\eta=0.16+0.84 \eta_{\mathrm{E}} \eta_{\mathrm{Th}} .
$$

As before, if we assume $\eta_{T h}=0.4$ and $\eta_{\bar{E}}=0.6$ to 1,0 , the overall energy-conversion efficiency for the radiolysis/electrolysis hybrid system is 36 to $50 \%$.

Current natural-gas costs are abovt $\mathbf{S I}$ per million Btu but may climb to $\$ 3$ per million Btu before the end of the century. ${ }^{5}$ The present estimated cost of synthetic gas from coal gasification plants is about $\$ 4.5$ per million Btu for the current process to as low as $\$ 3.5$ per million Btu for future plants. ${ }^{6}$ /n situ coal gasification may be able to produce gas at a cost of about $\$ 2$ per million Btu. 7

The cost of hydrogen produced by the various laser-fusion-driven processes is at least equal to the cost of energy from the fusion reactor divided by the overall energy-conversion efficiency. For comparison, assume a laser-fusion power plant produces alce :icity at a cost of 25 milis $/ \mathrm{kWh}$. (This is comparab: to the estimated cost of electricity from LWR and coal stations ordered in 1976 and online in 1985, quoted in 1976 dollars.) Also, assume th ithe capital component of the electricity $\cos 1$ is $80 \circ$ of the total and that the electrical power generation equipment comprises. $20 \%$ of this capital cost. (The turbine generators and building comprise about $30 \%$ of a typical LWR capital cost.) ${ }^{8}$ At a therm 1 -to-electrical conversion efficiency, $\eta_{\text {Th }}$, equal to 0.4 , the cost of energy is $\$ 2.93$ per million Btu for processes requiring electrical energy and $\$ 2.46$ per million Btu for processes requiring only tt rmal energy.

Table 5-1 gives the source-energy component of he cost of hydrogen produced by the various processes. These values reflect only the cost of using a laser-fusion reactor as the source of energy for the various processes. Additional costs will vary for the different processes depending on the cost of the components required. Large electrolysis units, for example, will add approximately $\$ 1$ per million Btu to the cost of hydrogen. 6

\begin{tabular}{|c|c|c|c|}
\hline & $\begin{array}{c}\text { Cost of sc urce } \\
\text { energ } \\
\text { (S/million Btu) } \\
\end{array}$ & $\begin{array}{c}\text { Energy conversion } \\
\text { efficiency }\end{array}$ & $\begin{array}{l}\text { Energy soures } \\
\text { component of } \\
\text { hydrugen cost } \\
\text { (\$/million Btu) } \\
\end{array}$ \\
\hline Thermochemical & 2.46 & $0.30-0.65$ & $3.80-8.20$ \\
\hline Electrolysis & 2.93 & $0.24-0.40$ & $7.30-12.20$ \\
\hline Radiolyás & 2.46 & $0.16-0.38^{b}$ & $6.50-15.40$ \\
\hline Radiolyais/electrolysis & 2.93 & $0.36-0.50$ & $5.90-8.10$ \\
\hline
\end{tabular}

Table 5-1. Energy source component of the cost of hydrogen.

"Does not include cost of chemical processing plant, clectrolysis units, or radiolysis components required.

$b_{0.16}$ and 0.38 correspond to $G$ values of 13.6 and 20 , respectively. 
It is clear there is no economic incentive for the production of synthetic fuels with laser fusion at the current price of natural gas, nor at the projected cost of gas from coal gasification. Even so, such a system may eventually be required. Oil and natura] gas currently account for about $75 \%$ of this nation's energy consumption. As these fuels are depleted the ability to produce large quantities of portable fuel will become a necessity. Laser-fusion-driven hydrogen-production processes represent a virtually inexhaustible supply of portable fuel.

\section{References}

1. S. S. Penner and L. Icerman, Energy: NonNuclear linergy Technologies (Addison-Wesley Publishing Co., Inc., Reading, Mass., 1975).

2. V. C. Rogers, G. M. Sandquist, and R. Baird, "Radiolytic Hydrogen Production from a Laser
Fusion System," Procepdings, Eleventh Intersaciely Energy Conversion Engineering Conference (Nevada, 1976), published by the American Institute of Chemical Engineers, N.Y.

3. Hydrogen Energy, R. N. Verziroglu, Ed. (Plenum Press, New York, 1975).

4. N. Vegelatos, et al., Radiolytic Production of Hydrogen Using Laser Fusion, IRT Corporation, IRT 7020-001 (1976).

5. The Energy Daily (Washington, D. C., April 15 , 1977). Vol. 5, 74.

6. Nuclear Power Issues \& Choices, S. M. Kenny. Jr., Chairman (Ballinger Publishing Co., Cambridge, Mass., 1977).

7. An In-Depth Evaluation of I.LL's R\&D Pragram for the In Situ Gasification of Deep Chal Seom, Lawrence Livermore Laboratory, Livermore, Calif., TID-27008 (1976).

8. W. Allen, Bechtel Corporation, San Francisco, Calif., private communication (May 1977). 


\section{PROPULSION SYSTEMS}

As the cost of the fossil fuels generally used for transportation continues to increase, the demand for nuclear fuels will also increase. To date, fission power is the primary nuclear fuel, but as fusion power develops, projections for its use in nuclear propulsion look more hopeful. The form of fusion best suited to propulsion requirements would be laser fusion. A laser fusion propulsion system would weigh less and be less bulky than its magnetic-fusion counterpart and less environmentally hazardous than a fission reactor.

There has been a trend in nuclear-fission power applications towards building fission-fueled propulsion systems, primarily for ships, and there is no reason ic expect that fusion power will not follow the same trend. Any nuclear-fueled propulsion system must compete with fossil-fueled systems, and, until recently, this has been quite difficult. The increase in the price of oil however, points more and more to the development of nuclear-propulsion systems.

Presently, about 250 shipboard nuclear-fission systems exist throughout the world. Projections, even before the recent inflation in oil prices, predicted 300 to 500 nuclear systems by the year 2000. 1 Trends also point to the likelihood of extraterrestrial propulsion systems for earth-orbit shuttles, earth-lunar missions, and interplanetary travel. Applications to aircraft are also under consideration. Table 6-1 details the characteristics of these kinds of systems.

jJuclear systems of any kind present a potential environmental hazard. In this respect, however, fusion systems have an advantage over fission systems because they use and produce smaller amounts of radioactive elements. The radiation hazards in fusion systems are primarily the tritium supply and radionuclides in the blanket material. A $0.1-\mathrm{kg}$ mass of tritium in the fusion fuel supply amounts to $10^{6}$ curies of uniquely low level biological hazard activity, but that level represents around 1.5 million kWh of usable fusion energy. The blanket material only has $10^{7}$ curies of radionuclides and represents a nonvolatile, localized hazard. A fission reactor, on the other hand, has $10^{10}$ curies of very volatile fission products for a power rating of $10^{9} \mathrm{~W}_{1}\left(3 \times 10^{5}\right.$ mechanical hp.) ${ }^{2}$

Nuclear-power propulsion systems will become more and more attractive as the price of oil rises, and less environmentally serssitive countries might pursue such alterratives vigorously, Ultimately fusion, fission and fossil powered systems will have to compete on a system by system basis. Between fu- sion approaches, laser- or inerticl-confinement fusion is considered to have the most potential for propulsion systems because specific weight and volume are the primary design parameters. In this regard, laser-fusion systems potentially have more flexibility in the tradeoff between combustionchamber firing tate and microexplosion yield.

Marine applicatinns of laser fusion appear the most feasible at this time. Much of the considcrable experience gained in marine nuclear-fission propulsion is applicable to the fusion-system concept. Both fission and fusion systems have high technology costs, high fixed costs, and low fuel costs relative to fossil systems. Fusion-fuel costs for material consumed will be negligible, but production costs might be quite high.

Until two years ago (before the dramatic increase in oil prices), clear economic advantages could not be made for "going nuclear"; now however. the case can be stated fairly simply 1 :

- Cost of constructing nuclear propulsion systems will drop as more efficient production methods are employed (e.g.. less one-of-a-kind, hand fabrication).

- Fossil-fuel prices for conventional systems are projected to rise substantially (inflation aside) over the next 10 or 15 years.

- Nuclear-powered ships can be built larger and faster to meet increasing consumer demands, particularly in container shipping.

From the national point of view:

- The use of nuclear propulsion decreases our dependence on overseas supplies. Fewer U. S. dollars will be spent on fuel abroad.

The United States would recapture its technological leadership in nuclear energy. Sales of nuclear propulsion units to others would again be an earner of foreign exchange.

- Fossil fuels are in "short" supply. The gradual changeover to nuclear propulsion will reduce our consumption of oil.

- Nuclear power (and fusion in particular) is essentially clean power, reducing pollution caused by fossil-fuel combustion. Moreover, the prime candidates for nucles propulsion are ships of high horsepower-larger, faster ships. This inplies a reduction in the absolute number of ships needed to carry any given cargo tonnage, a situation tha: has safety and environmental advantages.

The purpose of this chapter is to identify potential roles or missions for future laser-fusion propulsion systems and to briefly discuss the major 
engineering features of such systems. This task requires some foresight into the military-industrialcommercial transporation world of circa 2000. These projections shouid be viewed with caution because of the many pitfalls associated with such a task.

\section{Engineering Considerations For Fusion Propulsiun}

In general, a fusion-reactor power plant for a propulsion system must be designed for minimum specific volume and mass. Thus, the reactor will operate with a high first-wall loading. A laser-fusion reactor system is superior to a magnetic-fusion system because its specific volume and mass can be easily reduced by increasing the pulse-repetition fre. quency and the first-wall loading. Details on the specific volumies and masses for several types of propulsion applications are shown in Table 6-1.

Fusion gain $\eta_{\mathrm{L}} \mathrm{Q}$ (the ratio of the thermonuclear yield to the electric power driving the laser system) must be large for a reasonable system efficiency and a sniall specific volume and mass. For anticipated laser-system efficiencies, the volume and mass of the laser system per unit of power input to the laser (power stored) is roughly constant. Thus, the specific volume and specific mass of the laser system, including the E-beam machine, capacitor banks, and fluid-bandling equipment will be

$$
\begin{aligned}
& \frac{V}{P_{t}}=\frac{V}{P_{L i n}} \cdot \frac{P_{L i n}}{P_{t}}=\frac{V}{P_{L i n}} \frac{1}{\eta_{L} Q}, \\
& \frac{m}{P_{t}}=\frac{m}{P_{L \text { in }}} \cdot \frac{1}{\eta_{L} Q} .
\end{aligned}
$$

Therefore, the larger the fusion energy gain, the smaller the laser system specific mass and volume.

Reactor-chamber optical and first-wall materials, in which the short-ranged microexplosion energy is deposited, must have long lifetimes to increase the vehicle arailability and decrease the operating costs. Shielding must be provided to protect cargo, as well as passengers, from the high-energy neutrons. In addition, the react or system and other vehicle components that are subject to neutron activation or that collect activated material must be easily accessible and designed for remote maintenance.

Although these considerations will not be fully discussed in this report, they must be considered in designs of fusion-propulsion applications.

\section{Tarrestrial Laser-Fusion Propulsion}

Terrestrial propulsion systems, including marine and rircraft propulsion, will probably operate on a parasitic, DT fuel cycle using tritium produced in larger stationary fusion reactors. The first wall of the reactor will be a $d_{r y}$ wall and will either be magnetically protected or covered with a sacrificial liner. The use of a wet wall or fluid wall will be possible only if the reactor is mounted on a stable platform.

The reactors will operate on a Brayton cycle with helium, rather than a liquid metal as a cooling medium to minimize the hazard in the event of an accident.

The blanket for thermalizing the 14-MeV neutrons will consist of graphite with cooling passages for the helium. The porosity of the $2-\mathrm{m}$ thick blanket will be about $50 \%$, and it will be

\begin{tabular}{|c|c|c|c|c|}
\hline \multirow[b]{2}{*}{ Application } & \multirow[b]{2}{*}{$\begin{array}{c}\text { Technical } \\
\text { risk }\end{array}$} & \multirow[b]{2}{*}{ Power } & \multicolumn{2}{|c|}{ Constraints $^{2}$} \\
\hline & & & $\begin{array}{l}\text { Maximum } \\
\text { specific } \\
\text { mass }\end{array}$ & $\begin{array}{l}\text { Maximum } \\
\text { specific } \\
\text { volume }\end{array}$ \\
\hline $\begin{array}{l}\text { Marine } \\
\text { Navy } \\
\text { Commercial }\end{array}$ & $\begin{array}{l}\text { Moderate } \\
\text { Low }\end{array}$ & $\begin{array}{l}90 \mathrm{MW}_{\mathrm{s}} \\
90 \mathrm{MW}_{\mathrm{s}}\end{array}$ & $\begin{array}{l}30 \mathrm{~kg} / \mathrm{kW}_{\mathrm{B}}^{\mathrm{s}} \\
90 \mathrm{~kg} / \mathrm{kW}_{\mathrm{g}}\end{array}$ & $\begin{array}{r}80 \mathrm{~m}^{3} / \mathrm{MW}_{\mathrm{s}} \\
200 \mathrm{~m}^{3} / \mathrm{MW}_{\mathrm{s}}\end{array}$ \\
\hline $\begin{array}{l}\text { Airborne } \\
\text { Space }\end{array}$ & $\begin{array}{l}\text { High } \\
\text { Highest }\end{array}$ & $\begin{array}{l}130 \mathrm{MW}_{\mathrm{s}} \\
130 \mathrm{GW}_{\mathrm{t}}\end{array}$ & $\begin{array}{l}0.4 \mathrm{~kg} / \mathrm{kW}_{\mathrm{s}} \\
0.05 \mathrm{~kg} / \mathrm{kW}_{\mathrm{t}}\end{array}$ & $\begin{array}{l}1.5 \mathrm{~m}^{3} / \mathrm{MW}_{\mathrm{s}} \\
\text { Not critical }\end{array}$ \\
\hline
\end{tabular}

Table 6-1. Summary of propulsion system characteristics.

Meximum permitted for competitivity with other options. Comversion factors: $1 \mathrm{~kg} / \mathrm{kW}=1.6 \mathrm{lb} / \mathrm{hp} ; 1 \mathrm{~m}^{3} / \mathrm{MW}=26 \mathrm{ft}^{3} / \mathrm{khp}$. 
divided into two zones of equal thickness. The inner half of the blanket will be graphite to thermalize the neutrons. The outer half of the blanket will be $\mathrm{B}_{4} \mathrm{C}$ which will thermalize and capture the remaining neutrons.

For the blanket described above, the specific volume and specific mass are, respectively,

$$
\frac{V}{P_{P}}=\frac{P_{t}}{P_{P}} \sqrt{\frac{P_{t}}{36 \pi \phi^{3}}}\left(1+\sqrt{\frac{16 \pi \phi}{P_{t}}}\right)^{3} \frac{m^{3}}{M W_{S}}
$$

and

$$
\frac{\mathrm{m}}{\mathrm{P}_{\mathrm{p}}}=1.5\left(\frac{\mathrm{v}}{\mathrm{P}_{1}}-\frac{\mathrm{P}_{\mathrm{t}}}{\mathrm{P}_{\mathrm{P}}} \sqrt{\frac{\mathrm{P}_{1}}{36 \pi j^{3}}}\right) \frac{\mathrm{kg}}{\mathrm{kW}},
$$

where $P_{l}$ is the reactor thermal power, $P_{P}$ is the propulsion power, and $\phi$ is the first-wall total energy flux in $\mathrm{MW} / \mathrm{m}^{2}$. The lifetime of a graphite first wall liner is ${ }^{3}$

$$
\tau=\frac{2000 N}{P_{t} \phi^{S / 4}} \text { years }
$$

where $\mathrm{N}$ is the pulse repetition frequency (PRF). Thus, to minimize the specific volume and mass, it is desirable to operate with a maximum first-wall flux. To achieve a long lifietime with a given power and flux, it is desirable to operate with a small microexplosion and a high PRF. The size of the microexplosions and reactor gain decrease with a decrease in the laser-output energy. Thus, a reactor performance can be determined explicitly for a specific mass, volume, and lifetime. This reactor performance must then be checked with laser energy-pellet gain relationships to determine that the reactor performance will satisfy the total-system power requirements.

Approximately two-thirds of the tritium used in the pellets will not be burned. The unburned tritium from the microexplosions must be collected by a vacuum system and stored aboard the vehicle while it is in transit. This requirement is based on the cost of tritium, as well as the radiologica hazards associated with the tritium.

Advanced systems may directly convert the charged-debris energy to drive the lasers and the vehicle auxiliaries. The neutron energy will be used to drive the turbines using a Brayton cycle.

\section{Marine Laser-Fusion Propulsion}

Laser-fusion propulsion units in ships are probably the most realistic application at this time. Fission-powered ships are already in wide use, and laser fusion reactors have environmental advantages over their fission counterparts. Any nuclear system must be able to compete economically with fossil fuel systems, and, with the continuing rise in the price of fossil fuels, laser-fusion promises to do this. It has the additional advantage that ships need not stop in foreign ports to refuel.

The technology involved in marine laser-fusion applications closely follows the development of fission marine propulsion systems. Design requirements for fusion-propelled ships are based on what has been learned froin the widespread fission marine technology.

Requirements, however, differ according to the needs ' of the ship owners. Ships intended for military use have different requirements than those intended for commercial use (see Table 6-1). Military ships need to be fast and maneuverable, with low specific-weight and specific-volume propuision systems. Commercial uses tend to emphasize the economic aspects of the laser-fusiondriven propulsion units.

Naval Propulsion Systems. Superconductivity will strongly influence Navy power in the future because, above about $20,000 \mathrm{hp}$, it provides the minimum weight, space, and component systems to convert mechanical to electrical energy and viceversa. This will provide more flexibility and more operational freedom. With the development of superconductivity and fission-power technologies, the stage will be set for laser fusion in Navy propulsion systems.

A number of new ship concepts will be tested by the Navy in the next decade. Successful designs will be placed in service near the end of this century and potentially could be propelled by fusion-power plants. Table 6-2 lists these ship concepts and their planned displacement and speed ranges. Figure 6-1 shows the SWATH ship configuration. 4 Other ships listed are familiar and are not shown.

The shaft horsepower (shp) requirement for each ship concept varies considerably and depends on internal design features, such as weapons/sensor suite and atuxiliary power loads. Figure $6-2$ shows the specific power requirements of the ships from which shaft horse power (shp) requirements can be identified. The shp is related to the cube of the speed for conventional displacement vessels so that very sophisticated propulsion systems will be needed to meet such a range of requirements. By the year 2000, Navy vessels will require higher speed 
Table 6-2. Ship types under consideration by the Navy.

\begin{tabular}{|c|c|c|c|}
\hline & $\begin{array}{c}\text { Displacement, } \\
\text { tons }\end{array}$ & $\begin{array}{c}\text { Speed, } \\
\text { knots }\end{array}$ & $10^{\text {shp }}$ hp \\
\hline \multirow[t]{3}{*}{ Conventional displacement } & $>50,000$ (carrier) & $<35$ & 250 \\
\hline & $>10,000$ (escort) & $<35$ & 100 \\
\hline & $>3,000$ (patrol) & $<35$ & 40 \\
\hline \multirow[t]{2}{*}{ Small waterplene area twin hull (SWATH) } & $>10,000$ (cerrier) & $<35$ & 200 \\
\hline & $>3,000$ (escort) & $<50$ & 120 \\
\hline Hydrofoil & $<3,000$ (patroi) & $<65$ & 300 \\
\hline \multirow[t]{2}{*}{ Surface-effects ship (SES) } & 2,000 (patrol) & $<100$ & 140 \\
\hline & 8,000 (escort) & $\$ 100$ & 800 \\
\hline \multirow[t]{2}{*}{ Submarine } & $<10,000$ (FBM) & $<\mathbf{3 0}$ & 60 \\
\hline & $<4,000$ (attack) & $<50$ & 120 \\
\hline
\end{tabular}

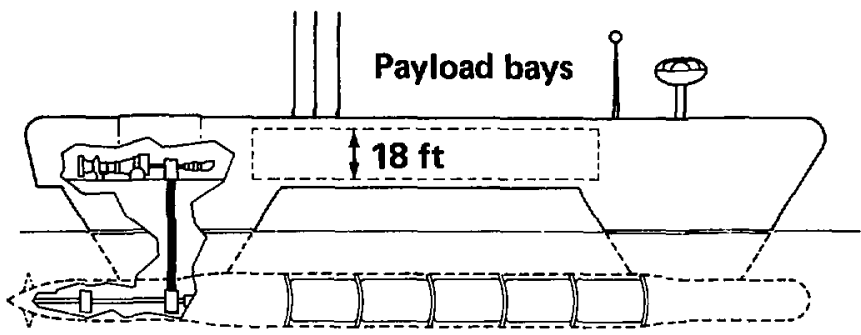

W.L.

\section{Propulsion Fuel/ballast}

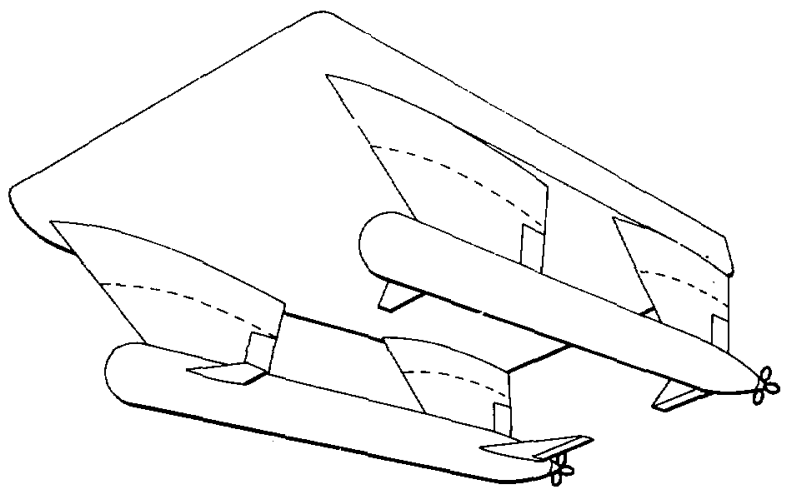

Fig. 6-1. Twin-hulled, semisubmerged ship configuration (SWATH). 
Fig. 6-2. Propulsiansystem specijic weights for Navy ressels.

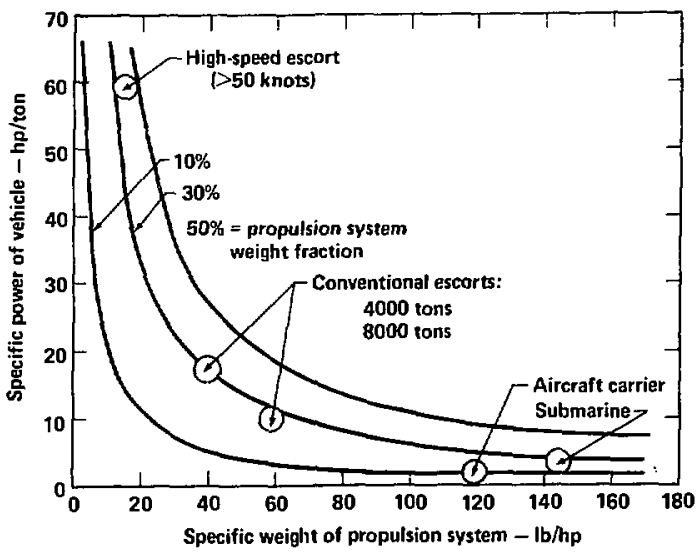

capabilities to retain certain attack options, and surface-effects ships (SES) or hydrofoils may be utilized. ${ }^{5}$ If the SES and hydrofoils are powered by nuclear reactors having low specific weight and volume ratios, they could exhibit essentially unlimited range, rapid response speed, and no need of fuel supply ships and foreign ports. Even advanced, high-powered fossil-fueled ships have a limiled range.
Figure 6.2 includes the fuel weight for conventional ships. Depending on endurance, a considerable amount of fuel is consumed on conventional ships that could be part of a fusion propulson plant of increased payload fraction (Fig. 6-3).

As indicated in Fig. 6-2, the propulsion-plant specific-weight objectives for an SES ship is 15 lb/hp. A 75,000-hp pressurized water reactor (PWR) power plant has been designed in a study for

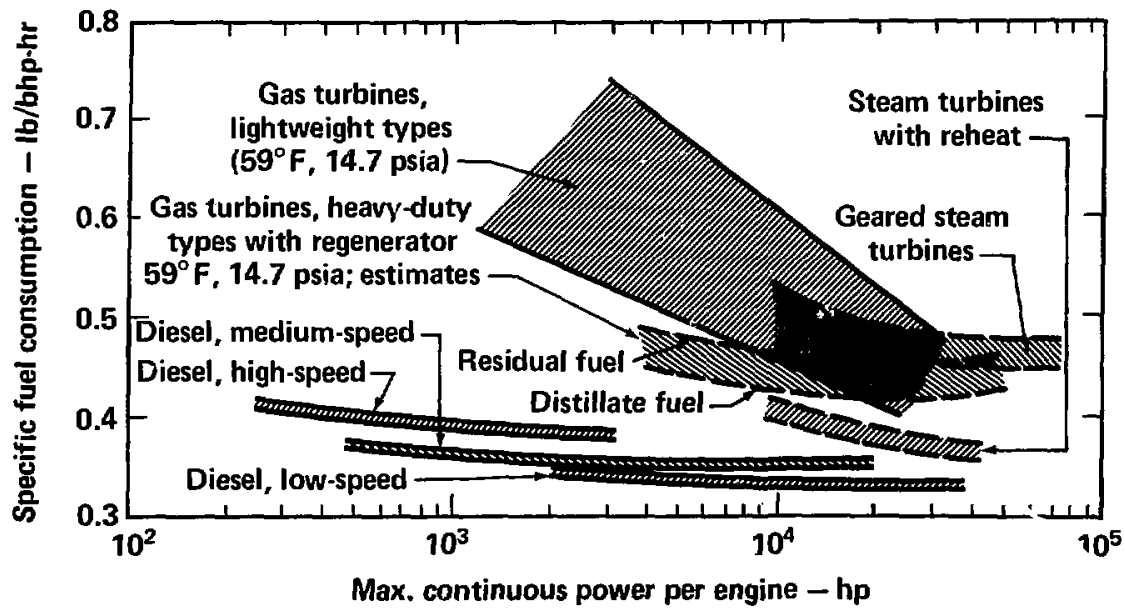

Fig. 6-3. Specilic fuet consumption for various types of marine power phats. 
merchant-marine applications with a specific weight of $100 \mathrm{lb} / \mathrm{hp}$. Various studies suggest that at 140,000 hp, an advanced PWR might be developed at $50 \mathrm{lb} / \mathrm{hp}$. This specific weight is not low enough for an SES. In fact, for the reference design, the steam-turbine package alone weighs more than the allowable weight for the total advanced power plant.

The prime power-conversion concept that is compact enough for a specific weight of less than 15 $\mathrm{lb} / \mathrm{hp}$ is a closed-cycle helium Brayton turbine with $1700 \mathrm{~F}$ and 1500 psia inlet of helium coupled with a very high-temperature, gas-cooled reactor. This should be available to the Navy for propulsion by the mid 1980's. Higher efficiency and tighter packing increasi the power-to-weight ratio, although they require a substantially different maintenance philosophy.

When attempting to specify future propulsion systems, it is of interest to estimate the power-plant specific-yolume requirements. Figure 6-4 shows typical specific volume values for conventional plants. These values, plus fuel volume for the endurance/speed needed, give an indication of total power plant volume.

The trend in naval propulsion is to greater shp and lower specific weights and volumes. Fusion systems, on this basis alone, must have performance goals that equal the minimum requirements for the larger combatants of the future. These ships will probably fall in the 10,000 ion displacement range and require 100,000 to $500,000 \mathrm{shp}$ at specific weights and volumes of less than $30 \mathrm{~kg} / \mathrm{kW}$ ( $(50$ $\mathrm{lb} / \mathrm{shp})$ and $80 \mathrm{~m}^{3} / \mathrm{MW}_{\mathrm{s}}\left(2 \mathrm{ft}^{3} / \mathrm{shp}\right.$ ). Presently, for instance, a 4000-ton nuclear submarine has a power-plant specific volume of $160 \mathrm{~m}^{3} / \mathrm{MW}_{\mathrm{s}}$ (4 $\mathrm{ft}^{3} / \mathrm{shp)}$.

An SES weighing 8000 tons and powered by four low-specific-weight nuclear units of $200,000 \mathrm{hp}$ each has been studied. It would have a payload capability of 1900 tons. For comparison, a fossil-fueled ship, carrying the same payload 3500 nautical miles with the same speed capability, would have a gross weight of about 17,600 tons and require at least 1.5 million hp. The lower ship cost and reduced fuel costs of the nuclear propelled ships are substantial economic advantages and are well worth development effort by the Navy. ${ }^{6}$

Commercial Marine Propulsion. Fusion propulsion systems for commercial marine applications, unlike those for military applications, are not built to the lowest specific weight or volume specifications. Economics are a much more important consideration. As before, the discussions about the potential for fusion-powered commercial ships are based on analyses and experience with nuclearfission propulsion.

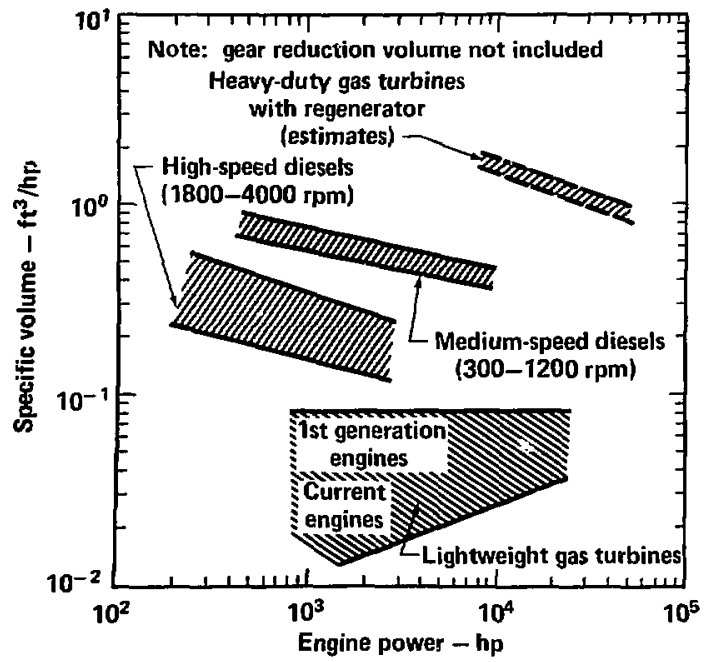

Fig. 6-4. Specific volume of various marine power plants. 
The commercial maritime industry has been $\mathrm{L} n$ able to sustain major research and development efforts, and, therefore, most important advances have been adapted from other industrial or military technology. Naval propulsion-machinery research and development programs have been particularly significant. The widespread application of nuclear fission power for stationary generating plants will also accelerate the acceptance of maritime nuclearfission power as well as the development of competitive shipboard installations. This trend witl probably hold for the introduction of fusion propulsion machinery as wetl.

The selection of ship size and speed are the most important design decisions confronting a prospective owner. Conventionally, with fossil-fuel systems, the speed has been kept lower than is commercially desirable because of the high costs required to increase them only slightly. There has been, however, a steady increase in the size of ships because larger sizes take only a little more por. Typical relationships between size, speed, and power for conventional bulk carriers are illustrated in Fig. 6-5. ${ }^{7}$ The dramatic increase in the power required for increased speed is clearly shown. Fusionpowered container ships and tankers will prohably be in the 250.000 to 450,00 dwt class with propulsion power of about $120,000 \mathrm{hp}$.

Fusion propulsion in the sea-going submersible cargo/tanker ship would be feasible in the 25- to 30 knot range. Below that, the speed-power ratio is too low to benefil from the addition of fusion propulsion.

Another future concept applies to tankers operating in icing conditions. It is projected that a single 400,000-ton displacement, semi-submerged tanker (Fig. 6-6, Ref. 8) could transport more than twice as much oil from the north slope of Alaska to markets on the East Coast than two conventional tankers. The propulsion requirement for this ship is, again, in the 125,000 shp range.

It appears that, if nuclear (fission) propulsion becomes competitive with fossil-fuel propulsion for merchant silps, the breakeven point will occur first for ships with the following characteristics :

(1) High power requirements: 100,000 to 120,000 . shp or more.

(2) High ship uilization:

(a) Short turn-around time in port, achieved through rapid loading and discharge of cargoes.

(b) Small numbers of Inading and discharge ports per voyagt.

(c) High load factors (i.e., a high ratio of cargo carried to available ship space (deadweight capacity).

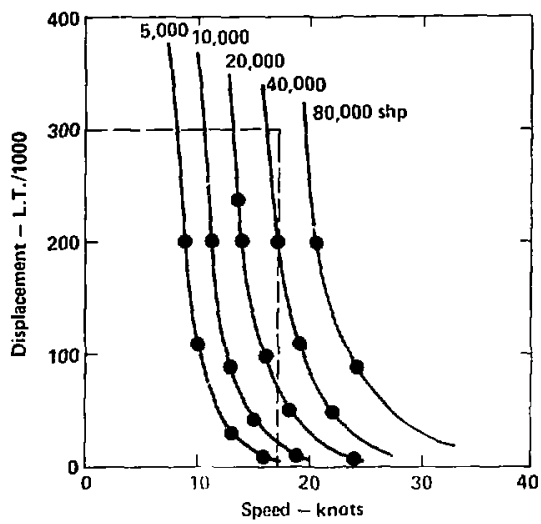

Fig. 6-5. Itisplacement rersus speed and shaft horsupuner for hulk carriers.

(3) Large (relarive) size: for containcrships or other quick-lurn-around liner vessels, 15,000 dwt or more: in the case of tankers, very large crude carriers (VLCC) or ultru-large crude carriers (ULCC), which range up ward, respectively. from about $150,000 \mathrm{dwt}$ and 300,000 dwt.

(4) Longer trade routes: the effect of greater size or speed will tend most to reduce the transport cost per ton of cargo.

(5) Subsidies: both construction-differential subsidy (CDS) and operating-differential subsidy (ODS). Subsidies not only reduce the shipowner's risk but have the effect of reducing the break-even point (i.e., the dollar-per-bbl price of bunker fuel oil for the conventionally powered ship) at which the higher capital costs of the nuclear-powered ship are offset by fuelcost savings.

These requirements will most likely hold for commercial fusion propulsion applications as well. Specific weight and volume requirements will be relaxed over those given for Navy ships by a factor of two or more.

Marine Propulsion Reactor Design. A marine fusion-reactor propulsion system will operate on a closed Brayton cycle. Assuming a thermal convertor efficiency of $35 \%$, for propulsion power of 120,000 shp, the reactor thermal power required will be 260 $M W_{t}$. In addition. assuming a power to the laser of $100 \mathrm{MW}$ and to the auxiliary equipment of $50 \mathrm{MW}$, the total thermal power required from the fusion reactor is $700 \mathrm{MW}_{1}$. The electric power for the laser 

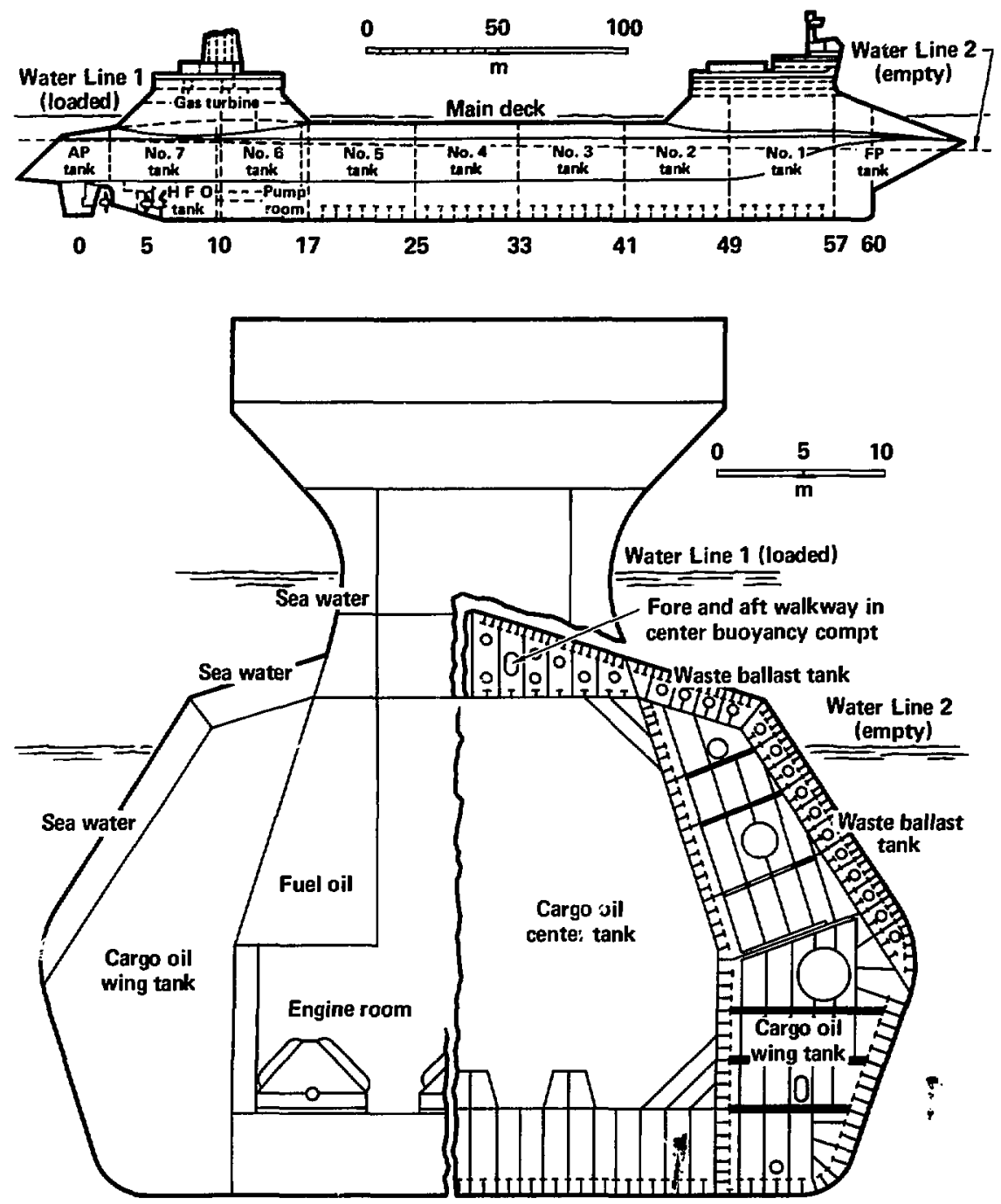

Fig. 6-6. Semisubmerged tanker configuration. 
and auxiliaries will be produced from a turboalternator.

The thermal power cycle can either consist of only a primary coolant cycle where the reactor coolant is circulated directly through the turbinecompressor system or a cycle where the primary coolant heats a secondary coolant, which is circulated through the turbine-compressor system. The trade:Af between the alternative power cycles is a smaller system specific volume and specific mass because of the elimination of a heat exchanger versus the requirement of remote maintenance for the turbine-compressor system.

The laser-system specific volume and mass are about $10 \mathrm{~m}^{3} / \mathrm{MW}$ and $0.3 \mathrm{~kg} / \mathrm{kW}$, respectively, based on the power input to the laser. The specific mass of the reactor system controls the design (for the nonoptimum system sclected). With a first-wall flux of $1 \mathrm{MW} / \mathrm{m}^{2}$, the specific volume and mass of the fusion-reactor system are $52 \mathrm{~m}^{3} / \mathrm{MW}_{\mathrm{s}}$ and 33 $\mathrm{kg} / \mathrm{kW}_{\mathrm{s}}$ respectively. The anticipated lifetime of the first wall is about 25 years for a pulse repetition freq! tency of $10 \mathrm{~Hz}$.

Based on these simple calculations for a nonoptimized, highly conceptual fusion-reactor system, it appears that laser fusion will be an interesting alternative to future chemical and fission energy sources for marine propulsion. Although, at the present time, there are no current military or civilian missions for marine propulsion in which fusion power has an advantage over fission or chemical power, the use of small inertially confined fusion reactors for marine propulsion appears to be feasible for a fusion energy gain of about 7. The use of fusion power for marine propulsion will stretch out our supplies of chemical fuels, allowing their use in ot her applications. such as ground and air transportation. Therefore systems studies on inertially confined fusion reactors for marine propulsion should be undertaken.

Note that the reactor proposed for marine propulsion also appears to be attractive as a small stationary power plant for isolated military and civilian applications. Therefore, the development of small stationary power plants can be a spinoff from. or sesult in the spinoff of, fusion-powered propulsion plants.

\section{Aircraft Propulsion}

As with marine propulsion, some experience gained in the development of fission reactors for aircraft jöopulsion can be applied to the development of fusion reactors. This experience is embodied in what remains from the Aircraft Nuclear Propulsion (ANP) project, which $x$ as, ters.linated in the early 1960's. This program was plagued with unpredictable technical problems associated with the helium and liquid-metal-cooled reactors of that era and with components, such as air-heat exchangers, bearings. pumps. seals, and the reactor-shield assembly. These problems, as well as uncertain cost benefits and, finally, public concern about the risk involved. led to the demise of the program. The latter problem is still of concern with aircraft nuclear propulsion. Fusion systems will not be totaily free of such worry.

The nuclear aircraft eventually may be able to compete with the costs of chemical aircraft in commercial cargo transport. particularly if depletion of petroleum resources drives the price of chemical fuel upward. With a substantial inheritance in $R \& D$ from military and national intcrest programs and continuing research, laser-fusion design parameters for a feasible aircraft propulsion system could be devejoped. In this regard, the following applications for long-range nuclear aircraft can be visualized for both land-based and sea planes:

Logistic aircraft employed for carrying commerical cargo and for rapid military-force deployment.

- Patrol aircraft for forward defense against submarine-launched ballistic missiles, terminal defense against ballistic missiles, command and control, warning and command, and ICBM launch surveillance.

- A strategic-missile carrier.

In this report, we discuss only the commercial cargo application. First, consider an existing 1. me aircraft, the Boeing 747-200F, with a gross weigh f about 300,000 lbs. The thrust, fuel consumption, and total aircraft power for the Boeing 747 are shown in Table 6-3. The maximum fuel load on the aircraft is 174 tons $\left(200 \mathrm{~m}^{3}\right)$. An inertially confined fusion-reactor system, including its negligible fuel supply, must have less mass than the fuel load to avoid a reduction in payload. (The gas turbine and compressor will be commnn to both the chemicaland fusion-powered systems, and the mass of the chemical combustion chamber is relatively negligible.) The specific mass and vol' ane of the largest mass and volume reactor systems that would not reduce the payload are shown in Table 6-4.

Larger cargo aircraft under consideration for nucleas-fission propulsion systems include planes with gross takeoff weights of 1,2 , and 10 million lbs. The I-million-lb system is comparable to the C-5A. Using data that incorporates the late 1960's low fossilfuel prices, the 1-million-lb nuclear plane would cost 6.4 cents per ton-mile. The same chemical 
Table 6-3. The thrust and fuel consumption of each engine on a Boeing 747-200F (GE-CF6-50D) and the power generated, based on $5.6 \mathrm{kWh} / \mathrm{lb}$.

\begin{tabular}{lll}
\hline & Cruise & Takeoff \\
\hline $\begin{array}{l}\text { Thrust, Ib/engine } \\
\text { Fuel consumption, } \\
\text { Ib kerosene/hr/engine }\end{array}$ & 11,000 & 50,300 \\
$\begin{array}{l}\text { Power - Total aireraft power } \\
\text { MW. }\end{array}$ & 7500 & 20,600 \\
$M W_{\text {: }}$ & 167 & 460 \\
\hline
\end{tabular}

plane would cost only 3.3 cents per ton-mile. The 2million-lb system would cost 3.9 cents for the nuclear and 2.6 cents per ton-mile for the chemical aircraft. In both sizes of aircraft, the nuclear system is more costly than the chemical propulsion system. The immense improvement in the cost of the 2million-lb nuclear system over the I-million-lb nuclear system results from its greater payload-togross-weight ratio.

The cost of the nuclear I-million-lb aircraft and the C-5A system are equal if a flight more than $5200 \mathrm{n} \mathrm{m}$ is flown nonstop with no aerial refueling and at a traffic level of 50 billion ton-miles per year. Equality for the 2-million-lb nuclear and chemical aircraft occurs at $3700 \mathrm{n} \mathrm{m}$. The reactor takes up relatively less space, leaving more cargo room, than does the fuel required for a long-distance chemical flight.

It is interesting to note that Japan's Shin Meiwa Industry Company is considering building a $1.04 \times$ $10^{6}$-lb-gross weight chemically fueled, over-ocean transport seaplane carrying 1200 passengers. ${ }^{9}$ The speed will be Mach 0.85 for a range of $3500 \mathrm{n} m$.
Table 6-4. Maximum specific mass and weight of an ICF reactor mounted in a Boeing 747.

\begin{tabular}{ccc}
\hline & Cruise & Takeoff \\
\hline Specific mass & & \\
$\mathrm{kg} / \mathrm{kw}_{\mathrm{t}}$ & 0.9 & 0.3 \\
$\mathrm{~kg} / \mathrm{kw}_{\mathrm{s}}$ & 3 & 1 \\
Specific volume & & \\
$\mathrm{m}^{3} / \mathrm{Mw}_{\mathrm{i}}$ & 1.2 & 0.4 \\
$\mathrm{~m}^{3} / \mathrm{Mw}_{\mathrm{B}}$ & 3.8 & 1.4 \\
\hline
\end{tabular}

Power requirements consist of six advanced highbypass-ratio turbofan engines in the $77.000 \mathrm{lb}$ thrust class. Unit costs are projected at $\$ 60$ million with a 7.4 cents per ton-mile.

Around the turn of the century, aircraft with gross weights of 10 million pounds may be considered feasible for bulk transport. Payloads of about 5 million pounds would allow inflight service and the plane would be grounded only for major overhaul. This continuously flying transport would virtually eliminate time lost in takeoff and landing and would, with VTOL feeder aircraft, permit passenger and cargo terminals to be located closer to city centers. The use of nuclear fusion rather than chemical aircraft would eliminate the need for aerial transfer of very large amounts of fuel. A 10-millionpound VTOL aircraft would also be desirable. l.aser-fusion systems are suitable for such highly variable thrust requirements because of the potential PRF flexibility they offer.

Table 6-5 gives an indication of the weights associated with the major components for the aircraft gross weights considered. As in the previous

Table 6-5. Weights of various components for the three sizes :- aircraft under tonside...tion.

\begin{tabular}{llll}
\hline & & Weights of aircraft & \\
\cline { 2 - 4 } \multicolumn{1}{c}{ Components } & 1 million lbs & 2 million lbs & 10 million lbs \\
\hline Cruise thrust, $k$ tb & 50 & 90 & 400 \\
Cruise power, MW, & 200 & 360 & 1600 \\
Power plant weight, tb & 95,000 & 175,000 & 700,000 \\
Fusion reactor weight, tb & 215,000 & 290,000 & 900,000 \\
Structure weight, tb & 425,000 & 845,000 & $3,400,000$ \\
Payload weight, tb & 265,000 & 690,000 & $5,000,000$ \\
\hline
\end{tabular}

ancludes all chemical expendables required for chemical-powered landing and takeofr. 
example for the Boeing 747, the fusion reactor mass is based or the chemical fuel load it displaces. These reactor masses and powers are converted to maximum allowable specific mass in Table 6-6.

The table shows that as aircraft size (and. hence. reactor size) increases, the allowable reactor specific mass decreases. However, since larger systems will use their mass more efficiently, this relationship is not immediately prohibitive. As shown earlier for fission powered aircraft, the smaller reactor systems probably sannot compete economically with chemically fueled aircraft because of their high capital cost per unit of power. The increase in fossil fuel prices over the last decade could change that evaluation: however, the higher cost estimated for fusion reactors compared to fission systems* will offset the chemical fuel price increase to some degree. Therefore, we consider the design of an ICF reactor for a two-million-pound aircraft. This design must have a specific mass of less than about $0.4 \mathrm{~kg} / \mathrm{kW}_{2}^{+}$to avoid displacing payload. A maximum specific volume constraint of about 0.5 $\mathrm{m}^{3} / \mathrm{MW}$, must also be satisfied, but it is more speculative because the reactor mass $A$ intribution will not match the chemical-fuel load distribution it replaces.

Aircraft Propulsion Reactor Design. A fusionreactor for an aircraft propulsion system will operate on an open Brayton cycle. Assuming a thermal-convertor efficiency of $35 \%$ for a cruise

\footnotetext{
* Based on cost estimates of a laser fusion hybrid electrical plant (Chapler 3).

tThe specific mass based on power supplied to the engines during cruising. Power recirculated to the fur in reactor and temporary power surges during takeoffs are not considered.
}

Table 6-6. Maximum fusion reactor specific mass for several aircraft gross weights.

\begin{tabular}{lllll}
\hline $\begin{array}{l}\text { Aircraft gross weight } \\
\text { (106 }\end{array}$ & 0.8 & 1 & 2 & 10 \\
$\begin{array}{l}\text { Total aircraft cruire power, } \\
\begin{array}{l}M w_{t} \\
\text { Maximum reactor specific } \\
\text { mass }\end{array}\end{array}$ & 0.9 & 0.5 & 0.4 & 0.25 \\
\hline
\end{tabular}

${ }^{\text {a }}$ Chemical fuel carried for chemically assisted takeoff is considered part of the payload. Alternatively, the ICF reactor may be operated at a higher PRF during takeoff, The specific mass shown is based on the nominal design power, i.e., cruise power. power of $125 \mathrm{M} \cdot$, the reactor thermal power will be $360 \mathrm{MW}_{1}$. For a laser input power of $95 \mathrm{MW}$ and auxiliary equipment input of $10 \mathrm{MW}$, the total thermal power required from the reactor is $650 \mathrm{MW}_{\mathrm{t}}$. The eleciric power for the lasers and auxiliaries will be produced from a curboalternator.

The energy from the reactor to the air passing through the compressor-turbine combination can, as for the marine-propulsion design, consist only of a primary cycle to the combustion chamber, or two loops from the reactor to the combustion chamber. The one-loop cycle is desirable because of its lower specific volume and mass.

For the reference reactor, the first-wall flux must be greater than $10 \mathrm{MW} / \mathrm{m}^{2}$ even if only the reactor (without the laser system) is to satisfy the maximum specific volume and mass constraints. If the effective density of the blanket material were somehow reduced by a factor of $S_{4}$ without degrading performance, the product of thermal-convertor efficiency and fusion gain must be $\mathbf{3 . 3}$ for the reactor alone to satisfy the maximum specific mass constraint with a first-wall llux of $10 \mathrm{MW} / \mathrm{m}^{2}$. However, the specific volume of the reactor alone would still be higher than the maximum permitted specific volume.

Thus, based on these kinds of calculations, it appears that a laser-fusion power plant for aircraft propulsion is not technically feasible because it will not be able to compete with chemical or fission systems on a specific mass or volume basis.*

\section{Extraterrestrial Laser-Fusion Propulsion}

Two primary applications of fusion propulsion in space are presently visualized; an earth-moon shuttle or ferry and an interplanetary spacecraft for manned missions. These applications can take advantage of the enormous gain in specific impluses relative to chemical rockets. Nucicons are accelerated in fusion reactions to velocities on the order of $10^{7} \mathrm{~m} / \mathrm{s}$, theoretically making inpluses on the order of $10^{6} \mathrm{~s}$ obtainable. ${ }^{2}$ Chs:mical rockets have specific impulses limited to less than $450 \mathrm{~s}$. Fission systems are limited to specific impulses of less than $2000 \mathrm{~s}$. 10

Spacecraft capability is normally measured in terms of $\mathrm{jV}{ }^{\dagger}$ This index is the sum of all propulsive energy required for translations betwe- $n$ stable orbits throughout the entire mission in units of feet

"This assumes that the same fraction of the aircraft is devoted to payload.

t This is the spacecraft $د \mathrm{~V}$. In the definition of specific impulse (given in the executive summary), $د v$ refers to the vclocity of the driving particles. 
per second. The $J V$ is a function of the specific impulse of the propulsion system and the payload and spacecraft structure fractions. Approximate $3 \mathrm{~V}$ 's for virious missions are given in Table 6-7.

( : ritual systems, because of their low specific impulies, have $\mathrm{JV}$ values most suitable to nearturlh operations. For example, if a chemical system uas designed to makc a manned Venus recon:alissance, the payload fraction would be negligible. $1 \cdot v e n$ with an advanced 51 ssion/chemical staged rucket (nuclear stage specific impulse of approximalely $1200 \mathrm{~s}$ ) the payload would only be about $2 \%$ of the total vehicle weight.

Reasonable translunar shuttle times should be on the order of 24 hours. $A$ fission sysiem with a $د V$ of $43.000 \mathrm{f} / \mathrm{s}, \mathrm{i} . \mathrm{c} ., 3,000 \mathrm{f} / \mathrm{s}$ more than required, could make the trip in the 24 hours specified. lusion systems with abundant $S V$ could make the trip much faster so that local mancuvers would consume a large part of the transit time. Such a highperformanee vehicle has been postulated using a laser-fusion propulsion system. Figure 6-7 gives the calculated performance for a $0.55 \times 10^{6}$ specificimpulse system which produces $130 \quad \mathrm{GW}_{1}$ and weighs approximately 300 tons.

\section{Reactor Design for Spacecraft}

A laser-fusion extraterrestrial propulsion system will consist of a sustained series of fusion microexplosions within an axially asymetric magnetic mirror (Fig. 6-8). The thrust of the vehicle is produced by the redirection of the charged plasma debris from the microexplosion through the larger of the mirror-loss cones and out the rear of the spacecraft.

The power plant of a specific-impulsemaximizing spacecraft bears very little resemblance to that used in terrestrial propulsion systems. Thrust is no longer produced as a by-product of a thermal cycle, but directly in the form of plasma debris from the fuel pellet. Neutrons and $x$ rays

Table 6-7. Spacecraft capability required for various missions.

\begin{tabular}{lr}
\multicolumn{1}{c}{ Mission } & $\Delta \mathrm{V}, \mathrm{f} / \mathrm{s}^{\mathrm{a}}$ \\
\hline $\begin{array}{l}\text { Escape from Eorth's gravity } \\
\text { Round trip from an Earth orbit }\end{array}$ & $40 \times 10^{3}$ \\
to the Moon's surface & $40 \times 10^{3}$ \\
Manned Venus reconnaissance & $150 \times 10^{3}$ \\
\hline
\end{tabular}

${ }^{a}$ Additional $\Delta V$ can be used during the mission to reduce the transit time. produced in the fireball are worse than useless: if intercepted. their energy must be rejected to space. The target design must maximize the ratio of ion energy to neutron and $x$-ray energy, while at the sime time minimizing required laser energy. Vehicle mass is dominated by the need for heat rejection to space-which must be done radiatively. Waste heat is developed in two ways: component inefíciencies and neutron and $x$-ray interception. The above factors dictate an unenclosed thrust chamber so that less than $2 \%$ of the solid angle seen by the $m \div$, roexplosion is occupied. Thus, while charged particles are redirected by the magnetic mirror providing thrust, most of the unwanted neutrons and $x$ rays escape to spuce harmlessly. Only $2 \%$ of the neutrons hit the superconducting-magnet radiation shields, payload shadow shiclds and laser beam mirrors. Even this $2 \%$ represents a large heat-rejection load. but it has some value because it permits tritium breeding in the lithium shields.

The optimal pellet is an intermediate $p \mathbf{R}$ design. mostly deuterium with just enough tritium to facilitate ignition. Such a pellet burns far more efficiently than low $p R$ pellets that require a smalier specific laser size. furthermore it allows laser reexcitation energy to be produced by direct conversion from the interaction of the charged plasma debris with an induction coil. Between 50 and $70 \%$ of the fusion energy is in charged debris from the microexplosion.

Hyde, Wood, and Nuckolls did two studies on rocket propulsion with laser-fusion microexplosions. 2.11 Their design utilizes heat-pipe radiation to dump the neutron and photon heat from the structure, radiation shields, and lasers into space. The lithium for tritium breeding is circulated using MHD pumps utilizing the existing mirror magnetic field.

Three major propulsion-system components are vulnerable to meteoroid damage. The laser and coil shield must be made almost invulnerable to meteoroid damuge: therefore, they are protected by a double-wall bumper shield. The heat pipes are protected by only a single wall. and the loss of a portion of them has been anticipated in the design.

Hyde, et al, estimates the total propulsionsystem mass to be 300 tons, distributed as shown in Table 6-8. About 112 tons of the laser-system mass and 14 tons of the thrust chamber mass are from their heat-rejection systems. Thus, the mass of the heat-rejection system constitutes $40 \%$ of the total mass of the propulsion system. The mass of the neutron shields in the thrust chamber is 20 tons while the mass of the coils in the thrust chamber is about 15 tons. 
Closest approach distances to Earth Jupiter Uranius

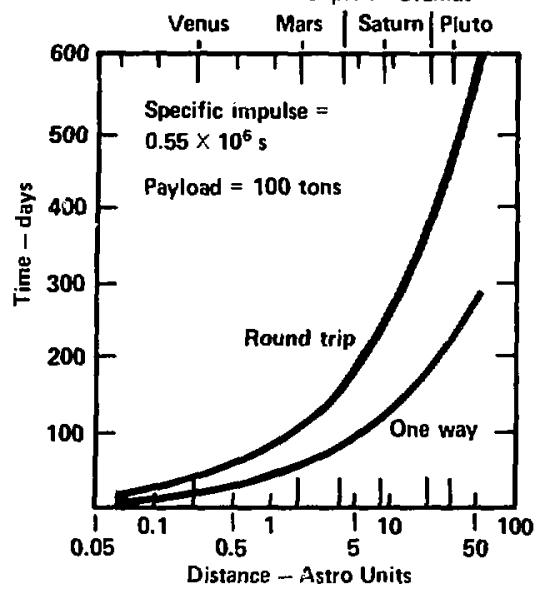

Fig. 6-7. I.usce-fusion-pasucred spaceceruft performance.

Table 6-8. Spacecraft fusion-propulsion-sy stem mass.

\begin{tabular}{lc}
\hline \multicolumn{1}{c}{ Component } & Mass, tons \\
\hline Proptulsion syst'm (total) & 300 \\
Laser systemt & 151 \\
Thrust clamber & 54 \\
Tinergy distributor & 17 \\
Refrigeration & 9 \\
Tanks & 25 \\
Miscellaneous parts & 44 \\
\hline
\end{tabular}

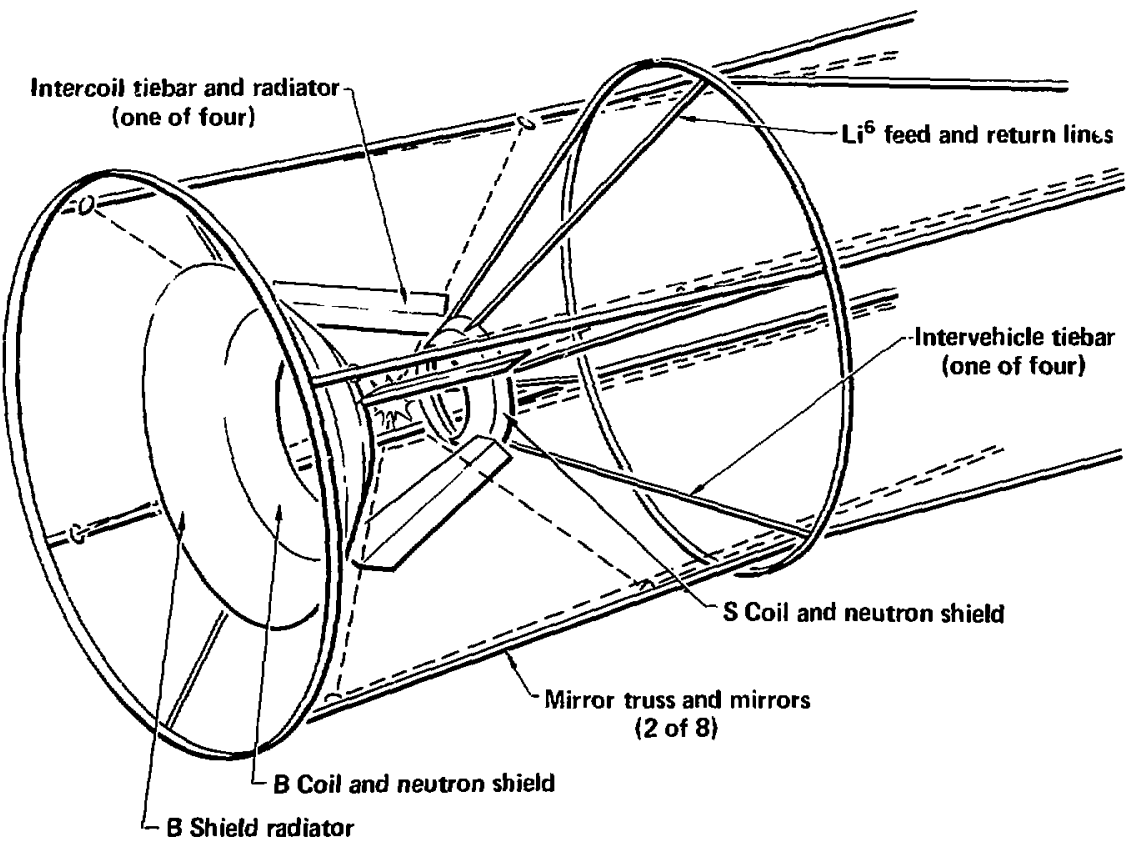

Fig. 6-8. Fxtraterrestrial vehicle thrust chamber. 
The specific-impulse-maximizing spacecraft study includes a pcllet design which requires $1 \mathrm{MJ}$ of laser light from a $33 \%$ efficient electron-beam-excited system. The total yield from the microexplosion is $260 \mathrm{MJ}$, with $120 \mathrm{MJ}$ producing thrust. The power needs of the propulsion system are almost exclusively those of thc laser because refrigeration and Li $^{6}$-pumping power are negligible. For a PRF of 500 , the system develops 2.2 tons of thrust at a specific impulse of $5.5=10^{5} \mathrm{~s}$, or an exhaust velncity of $6=10^{6} \mathrm{~m} / \mathrm{s}$ For a spacecraft with a 300-ton propulsion system and a 200-ton deuterium fucl load, a payload of 100 tons can make a round trip to any point in the solar system in one year. Interstellar missions of a few decades in transit time are also feasible with a laser-fusion propulsion sysiem.

\section{Conclusions}

Laser-fusion reactors for propulsion (as they are now viewed) would operate in a low-yield, highPRF mode with high first-wall fluxes. From inertial considerations, the first wall should be a dry wall rather than a wet or fluid wall. These reactors could operate on a parasitic fuel cycle from larger stationary fusion-power reactors.

Although there is an apparent need for advanced, low-fuel-cost propulsion systems, it is too early to determine if laser fusion will be an economical alternative to future chemical or fission plants for marine propulsion. Aircraft applications appear even more uncertain because of the stringent specific volume and mass technical constraints. Laser-fusion propulsion plants for extraterrestrial propulsion are possible, provided there is a demand for intrasolar system or intersteller missions.

Because marine propulsion appears to be the least demanding system with the earliest payolf, systems studies and conceptual designs of laser-fusion marine propulsion systems should proceed in tandem with the technical development of the program.
These studies should include the impact of a laserfusion reactor on hull design and potential technical spinoffs on the development of small staticnary power plants.

\section{References}

l. Nuclear Merchant Ships, National Academy of Sciences, Maritime Transportation Research Board (1974).

2. R. Hyde, L. Wood and J. Nuckolls, "Propulsion Applications of Laser-Induced Fusion Microexplosions," Proc. First Topical Meeting on ine Technology of Conirolled Nuclear Fusion, I, 159 (San Diego, Calif., 1974).

3. J. Hovingh, "First-Wall Costs of an Ion-Beam Fusion Reactor," paper given at summer Annual ANS Meeting, New York, (June 15, 1977).

4. P. Warnshuis, Logistic Applications for Semisubmerged Hullforms, NUC TN 1696 (March 1976).

5. R. J. Creagon, Pawer and Energy-Navy-2000 A.D., Marine Board of National Research Council (August 6, 1975).

6. F. R. Riddell, Effect of Specific Weight and Volume Changes in Power Machinery on the Size and Speed of Submarines-A Preliminary Analysis, IDA Paper P-1233 (December 1976).

7. Oceanborne Shipping: Demand and Technology Forecast-Part I, Litton Systems (June 1968).

8. "Semi-Submerged Tanker for Arctic Waters," The Naval Architect (Nay 1975).

9. A viation Week and Space Technology, p. 45 (May 16, 1977).

10. H. F. Crouch Nuclear Space Propulsion, Astronuclear Press (Granada Hills, Calif., 1965).

11. R. Hyde, I.. Wood, and J. Nuckolls, "Prospects for Rocket Propulsion With LaserInduced Fusion Microexplosions," AIAA/SAE 8ih Joint Propulsion Specialist Conference Paper 72-1063 (New Orleans, Louisiana, November 1972). 


\section{ACKNOWLEDGMENT}

We would like to acknowledge technical contributions from W. O. Allen. R. E. Aronstein. B. Marais and S. L. Thompson of Bechtel, and L. F. Hansen of L.L for participation in the hybrid design study (Section 3). R. Rose and his solleagues at Westinghouse provided most of the material on actinide burning (Section 4). The attractive appearance and layout of the report resulted from the efforts of $A$. Heine. M. Genin, and $K$. Minkel. 


\section{A DEVELOPMENT SCENARIO FOR INERTIAL CONFINEMENT FUSION}

\section{Long-Range Plan}

As the inertial-confinement fusion (ICF) program approaches a demonstration of scientific feasibility, it becomes increasingly important to prepare long-range plans. The plans should be able to accommodate optional technical approaches to commercial fusion-power production and provide tor varied paces of tcihnological development and application. The latter provision would allow management to keep the program in line with changes in technical progress, national needs, and the nation's commitment to inertial confinement fusion (ICF). The plan should also establish the goals and milestones required for commercial applications. Establishing goals would enable us to identify the requirements and tasks in the early (scientific) stages of development, working back from the application dates. Finally, we wanted a plan that would identify the major issues and decision points. Moreover, the achievement of these objectives completely defines the scope of our long-range plan.

In addition to these objectives, there were a few assumptions and guidelines that significantly influenced the development of this long-range plan for ICF.

- The ICF program will be competing with other long-term sources of energy, and we must anticipate major priority decisions.

- Commercial ICF power production will be developed by an industry that is working in conjunction wit fovernment, national laboratories, and utilities.

- The ICF program will go through several phases of research and development before commercial power is achieved. Key decisions will be required in each phase.

- A broad technology base must be established that will both feed and be fed by the various developments in the program.

The flow of logic for several optional paths to commercial fusion power is diagrammed in Fig. A-1. This logic is based on the premise that experiments to verify the scientific feasibility of initiating high-gain microexplosions are independent of the development of high-averagc-power ignition sources.

In addition. solid-state laser technology represents the most highly developed short-pulse, high-peakpower driver technology in existence. Consequently, the series of solid-state laser-fusion facilities at LLL (from Janus to Shiva Nova) is expected to provide the earliest understanding of implosion physics and demonstrate the scientific feasibility of ICF. Once the physics of inertial confinement fision are understood, and, after high-gain microexplosions have been demonstrated, the program can progress to the engineering phases of development and selection of the most promising high-average-power driver for an experimental power reactor. Potential ignition sources for high-average-power operation of the experimental reactor include ion beams. gas lasers. and electron beams.

\section{The Phases of Development}

A more comprehensive scenario for developing inertial-confinement fusion is presented in Fig. A-2. In this plan, the program progresses through several phases: exploratory development, advanced development, engineering development, and commercial development. The demonstration of technical feasibility has been split between the advanced and engineering phases because of the prevailing assumption that two experimental power reactors will be needed before a demonstration power reactor can be built. As shown, this longrange plan provides for optional technical paths to commercial fusion. Moreover, the plan is based on the premise that a broad scientific base must be established in the earliest phase of development and must then be allowed to operate through a technical framework that will both feed and be fed by the various developments.

\section{Exploratory Development}

Those criteria required to demonstrate the scientific feasibility of inertial confinement fusion will be met in the exploratory development phase. Scientific feasibility will be demonstrated when a high-gain $(G \mathbf{r}>20)$ 


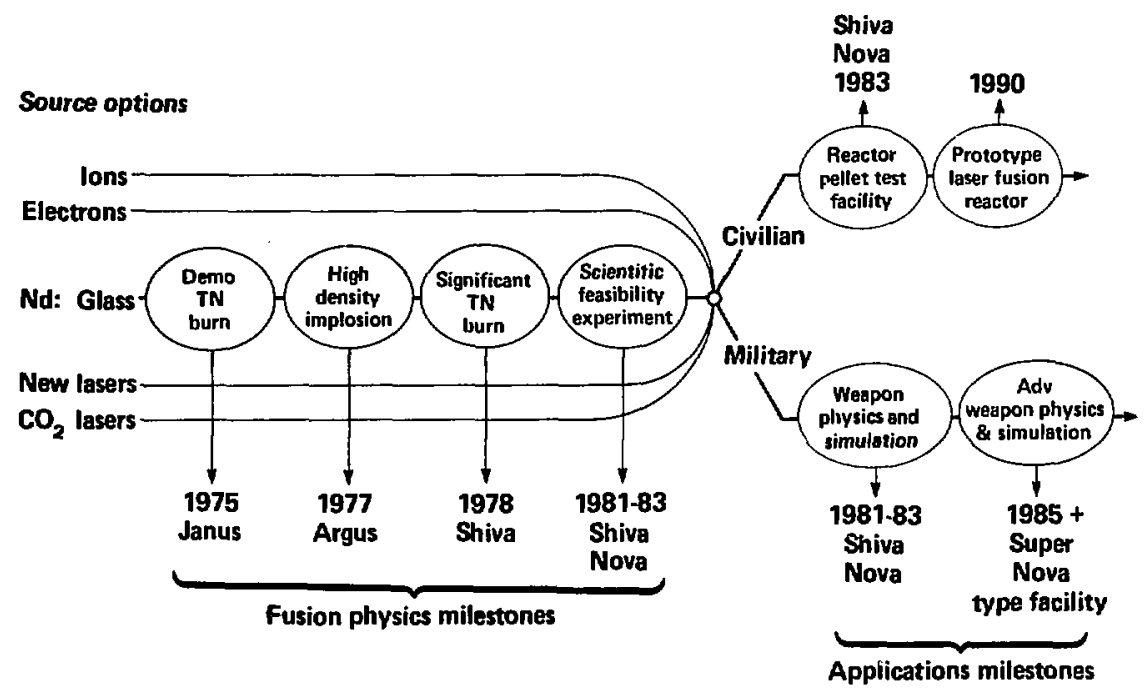

Fig. A-1. Iwertial continement fusion development logit.

thermonuclear microexplosion is achieved and a suitable high-average-power ignition source is developed. These accomplishments obviously must precede the decision to build an experimental power reactor.

The major program elements in the exploratory phase are suparated into three distinct segments: inertialconfinement physics, source-coupling physics, and source feasibility, Neodymium:glass lasers operating at $1.06 \mu \mathrm{m}$ with low-average-power capabilities will be the primary tools in the inertial-confinement physics program. Shiva Nova, the largest of the Nd:glass laser facilities is projected to provide the on-target energies and power required to drive pellets suitable for comenercial power plants on a single-shot basis. The Shiva Nova facility will also be capable of driving significant national security experiments.

The source-coupling physics program will be concerned primarily with quantifying and maximizing the efficiencies of coupling other forms of energy-different-wavelength lasers, ion beams, and electron beams-to the fusion target. In the source-feasibility program, several high-average-power sources (gas lasers, ion beams, and electron beams) will be developed and analyzed.

The facility for pellet development will support work in the exploratory phase by providing 10 pellets per day for Shiva Nova. This facility must also develop the techn-ulogy to produce pellets at the increased rates required by the first experimental power reactor (1000 pellets per day).

The major program elements initiated in this exploratory development phase will continue throughout the development of ICF, thereby forming a foundation of continually expanding technology. The decision to proceed into the next phases (advanced and engineering development) will be based on the successfui demonstration of a high-gain microexplosion and on the development of a suitable high-average-power driver.

\section{Adranced Development}

The first experimental power reactor based on the ICF concept, EPR 1, will be undertaken in the advanced development phase. This reactor will be the first of a series of major experimental facilities required to verify the physics and engineering principles that are required to commercially develop ICF. The primary function of EPR I will be to provide information on the integrated engineering performance of a complete 


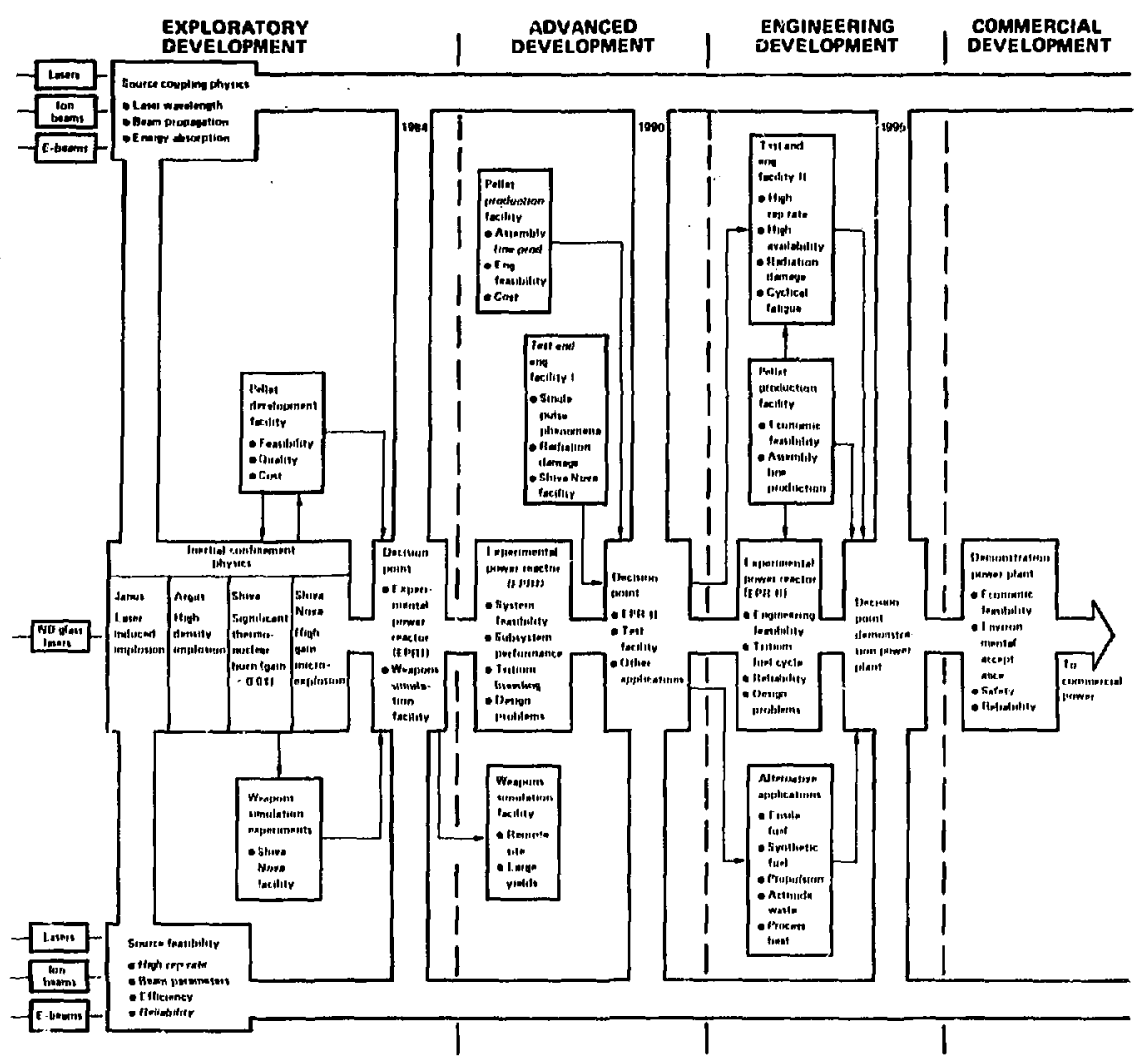

Fig. A-2. Phuses of development in the long-range plan for inertial confinement fuston.

ICF system (source, target, and reactor). In addition, EPR I will be expected to demonstrate the capability to produce electric power. An experimentul power reuctor supporting these objectives will:

- Operate at approximately $10^{3} \mathrm{pulses} / \mathrm{day}$

- Generate $\simeq 10 \mathrm{MW}$ of thermonuclear power

- Have limited plant availability $\propto 20 \%$

- Obtain a fusion-cnergy gain $>3^{*}$

- Achieve high-temperature operation

- Cost approximatcly $\mathbf{5 0 0}$ million dollars.

In the advanced development phase, pellet technology will consist primarily of producing 1000 pellets per day for EPR I. The capability to produce pellets even more rapidly ( 105 pellets/day) will huve to be developed to meet the requirements of the next experimental power reactor.

-Fusion-energy guin is defined is the product of pellet gain and laser-system elficiency. As suct it represents the fatio of thermonuclearenergy output to electricul-enetgy input. 
A test and engineering facility also will be built and operated to investigate the effects of the thesmonuclear environment on the finul focusing eleinents, the first wall, and the blanket structure. This facility will not have a high-average-power capability and therefore will be used to investigate single-pulse phenomena ( $<10$ pulses/duy). These test and engineering requirements could be met most cconomically by utilizing the present Shiva Nova laser fusion facility.

\section{Eingineering Development}

This third phase of the program will focus primarily on the major technical problems associated with an ICF power plant. These include the development of:

- A high-average-power driver with the required efficiency $(>1 \%)$ and reliability $(>70 \%)$.

- A first wall able to withstand the effects of $x$ rays, debris, and neutrons from the microexplosion. cyclical stresses.

Structural materials that can withstand the cumulative dumage effects of high-energy neutrons and

Final-focusing elements that can be placed far snough away from the microexplosion that they can last a reasonable length of time.

The technology to cheaply mass-produce the fusion targets.

The second experimental power reactor, EPR II, will be built to demonstrate limited stendy-state clectrical generation in a smaller and less costly mode than is available with a demonstration power reactor. The experimental power reactor thut can accomplish this objective will:

- Operate at $10^{5}$ pulses/day.

- Generate considerable thermonuclear power $\left(>100 \mathrm{MW}_{1}\right.$ and tens of $\left.\mathrm{MW}_{\mathrm{e}}\right)$.

- Incrrase plant availability to $>40 \%$.

- Achicve fusion-energy gain $>\mathbf{6}$.

- Demonstrate tritium regeneration and recovery.

- Cost approximately 1 billion dollars.

In addition, a test and enginecring facility will be started in this phase to qualify materials for commercial power-plant operation. This facility will:

- Generate $\approx 10 \mathrm{MW}$ of thermunuclear power.

- Consume $<100 \mathrm{MW}$ of electric power off-line.

- Achieve fusion-energy gain $>0.1$.

- Extend plant uvailability to $\simeq 70 \%$.

- Test materials for commercial operation to $10^{23}$ neutions $/ \mathrm{cm}^{2}$.

- Cost approximately 500 million dollars.

Long lead times are generally required to develop and test new materials. Therefore, it would be desirable to construct this high-average-power test facility much earlier in the program. Unfortunately, it is difficult to project a more rapid development of the technology that is required to operate at $70 \%$ plant availability.

The pellet production facilities in this phase will provide $10^{5}$ targets per day for both EPR II and the materials test facility. At this stage, we must develop the technology for a fuel factory to mass-produce targets cheaply enough to allow economical power production.

Nonelectric applications of ICF have been identified, including propulsion, fissile-fuel production, hightemperature process heat, burnup of rudioactive fission wastc, and synthetic-gas production. The technical feusibility and economic incentives for developing these alternative applicutions will be evaluated during this phase. The decision to pursue any of the alternative applications in conjunction with the production of commercial electricity must be made by weighing the recognized benefits against the additional costs for develepment.

\section{Commercial Development}

The major efforts in this final phase of development will be associated with planning, constructing, and operating a demonstration power reuctor. This fucility will be required to demonstrate the potential for economically produced ICF electricity over alternative long-runge sources of energy by operating in a safe, reliable, and environmentally acceptable manner. In shorl, the demonstration power reuctor (DPR) will:

- Generale 500 to $1000 \mathrm{MW}$ (several $100 \mathrm{MW}_{e}$ ) of thermonuclear power.

- Reach a plant availability $\approx 70 \%$.

- Breed tritium. 
- He fully licensed as a power reactor.

- Consume less than $25 \%$ of generated power as recirculating power.

- Achieve a fusion-energy gain >10.

- Cost less than 2 billion dollars.

\section{Time Schedules and Optionis}

For planning purposes, alternate time schedules for major enginecring facilitics are projected in lig. $\wedge$ -. . The three planning options are designated as cautious, aggressive (midrange), and accelerated.

In the ciatious plan. the decision to begin the design of a inajor enginecring facility is made only after the preceding facility has achicved the milestones required for scientific justificalion. For example, the planning and design phase of the I:P'K II would not hegin until liPK I had operilled and achieved its objectives. In this plan, technical progress is lionited by the availability of funds and the demenstration power plant would not operate until ufter the turn of the ecutury.

In the aggreskive plan. the decision to begin construction of a major enginecring lancility would mot be made until the preceding facility had achieved the inilestones required for scientilie justiliculion. Wills this logic, construction of liPK $\mid 1$ would not hepin until liPR 1 had ach oved its objectives. Jiunding is adequate and new projects are undertaken when their success is refosmably atssured. A demensiration plant would operate at the turn of the entury.

In the accelerated plan. the need for a new energy source is extremely urgent. Major engineering lacilities arc addressed in parallel, and new facititics are begun as soon as their meded is defined. Thus, planning for FPK I would begin in 1983, before the scientific leasibility of such a plant has been demonstraled. Reakenable scientific justification would be obtained by extrapoluting resulte Prom previous facilitics. I:unding is available

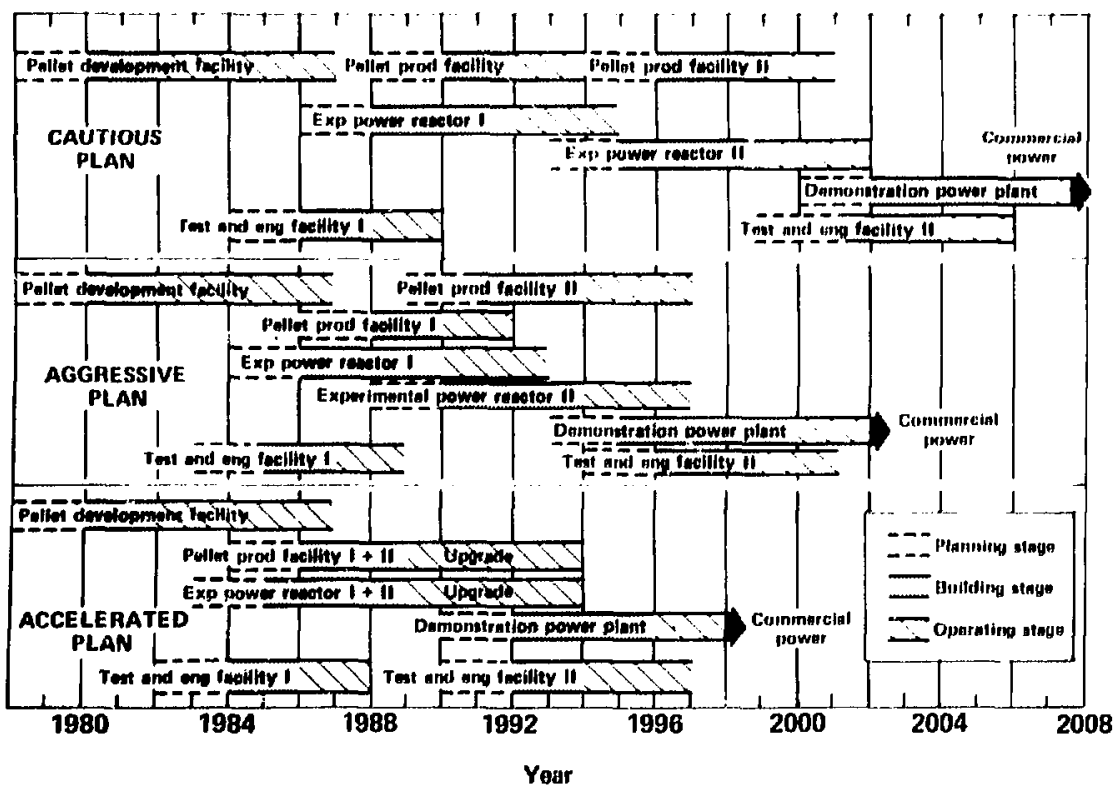

Flg. A.3. Fogleering Facilitien for three kong-range planming opulinn. 
and adcquatc for the construction of major facilities in parallel. following this plin, a demonstration plant would operate in the mid-1000s.

(omparing these three plans, we note that from the slowest to bisicst opfion. there is a diflerence of II yeats in the operational date of the demotstration power reateor. Iowever, in the accelerited plan, the decrease in tittic to the DPR is gaincd at the expense of parallel developsnemt of major facilitics. This high-risk stritcgy would seriously impuet the rate of spending in the program, as well tis the rate of employing the tcchnology base of maspower and production capabilitics.

In lig. $\$$-4, the time schedule for the enginecring fateilitics is the aggessive (anidrange) plan is presenled together with the time seliedule for the developuent and operalion of the required scientifie fincilities. Ilere we

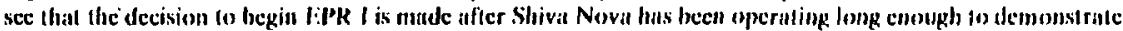
a high-gain microexplosion adeguatcly, and afler all of the potential high-average-power sources have beca operationg long enough to be evaluiled. Pellet development is the only major enginecring teclonology that strengly overlaps the design and construction of the scientilic lineilities. firom this, it is evident thut we must hegin to develon the technology to mass-preduce targets immediately so that it will be available: when the deci-

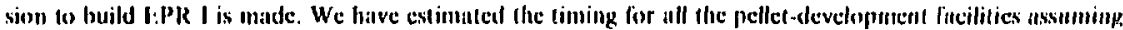
that current pellet technology can be aleveloped rupidly cnentgh to prevent pellet availability frum becoming. it pacing clentent in the programs.

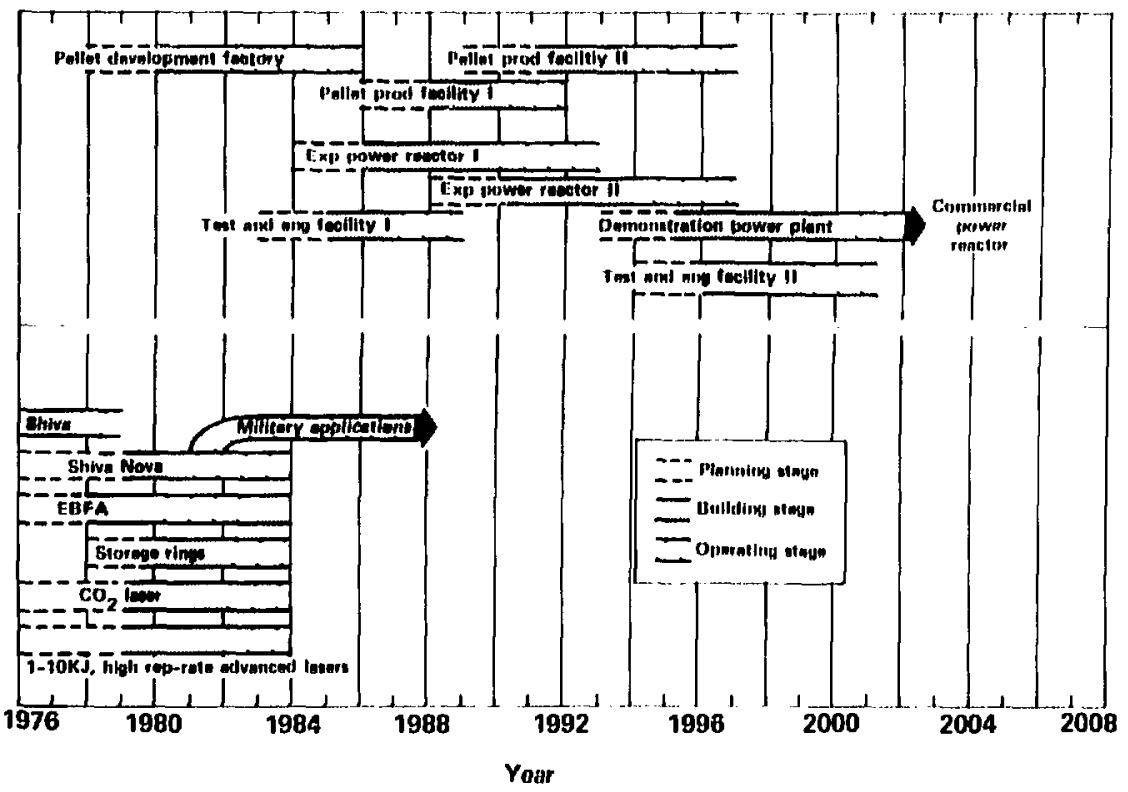

Fig. A.4. Sicknilfic and pnkinering facilities fur the akgrendive option. 